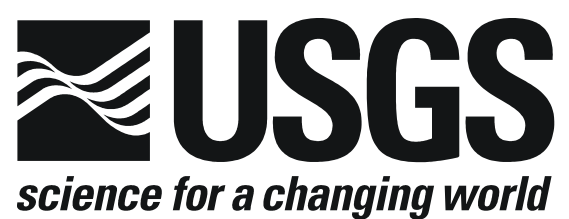

\title{
Magnetotelluric Data Across the Pajarito Fault, West of Santa Fe, New Mexico
}

By Jackie M. Williams and Brian D. Rodriguez

\section{Open-File Report 2005-1054}

U.S. DEPARTMENT OF THE INTERIOR

U.S. GEOLOGICAL SURVEY 


\section{U.S. Department of the Interior \\ Gale A. Norton, Secretary \\ U.S. Geological Survey
P. Patrick Leahy, Acting Director}

U.S. Geological Survey, Reston, Virginia 2005

Revised and reprinted: 2005

For product and ordering information:

World Wide Web: http://www.usgs.gov/pubprod

Telephone: 1-888-ASK-USGS

For more information on the USGS—the Federal source for science about the Earth, its natural and living resources, natural hazards, and the environment:

World Wide Web: http://www.usgs.gov

Telephone: 1-888-ASK-USGS 
Contents

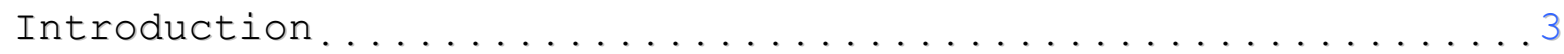

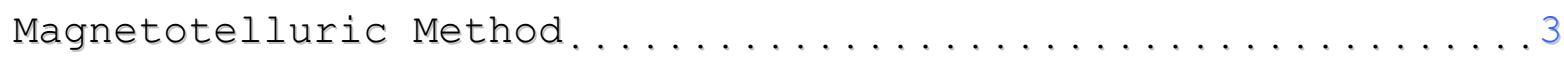

Magnetotelluric Survey......................4

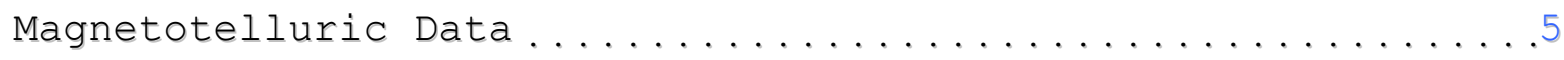

Reference Cited .........................8

Appendix Magnetotelluric Data Plots...................10 


\section{Introduction}

The Santa Fe region is rapidly growing. The Santa Fe Group aquifer, east of the Pajarito Fault, in the Española Basin is the main source of municipal water for the region (fig. 1), and water shortfalls could have serious consequences. Future growth and land management in the region depend on accurate assessment and protection of the region's ground-water resources. An important issue in managing the ground-water resources is a better understanding of the hydrogeology of the Santa Fe Group, the sedimentary deposits that fill the Rio Grande rift and contain the principal ground-water aquifers.

The U.S. Geological Survey (USGS) is doing a series of multidisciplinary studies of the Española Basin in northern New Mexico. Detailed geologic mapping, high-resolution airborne magnetic surveys, electromagnetic surveys, and hydrologic, lithologic, and hydrogeochemical data are being used to better understand the aquifer systems. A magnetotelluric (MT) survey was done as part of these studies. The primary purpose of the MT survey was to map changes in electrical resistivity with depth that are related to lithologic variations important to the critical aquifers across the Pajarito Fault. The purpose of this report is to release the MT sounding data; no interpretation of the data is included.

\section{Magnetotelluric Method}

The magnetotelluric (MT) method is a passive surface geophysical technique that uses the Earth's natural electromagnetic fields to investigate the electrical resistivity structure of the subsurface. The resistivity of geologic units is largely dependent upon their fluid content, porosity, degree of fracturing, temperature, and conductive mineral content (Keller, 1989). Saline fluids within the pore spaces and fracture openings can reduce resistivities in a resistive rock matrix. Resistivity also can be lowered by the presence of conductive clay minerals, carbon, and metallic mineralization. It is common for altered volcanic rocks to contain authigenic minerals that have resistivities 10 times less than those of the surrounding rocks (Nelson and Anderson, 1992). Increased temperatures cause higher ionic mobility and mineral activation energy, reducing rock resistivities significantly. Unaltered, unfractured igneous rocks are moderately to highly resistive (hundreds to thousands of ohm meters [ohm-m]), whereas fault zones will show low resistivity (less than $100 \mathrm{ohm}-\mathrm{m}$ ) when they are composed of rocks that are fractured enough to have hosted fluid transport and consequent mineralogical alteration (Eberhart-Phillips and others, 1995). Carbonate rocks are moderately to highly resistive (hundreds to thousands of ohm-m) depending upon their fluid content, porosity, fracturing, and impurities. Marine shales, mudstones, and clay-rich alluvium are 
normally conductive (a few ohm-m to tens of ohm-m). Unaltered, metamorphic rocks (nongraphitic) are moderately to highly resistive (hundreds to thousands of ohm-m). Tables of electrical resistivity for a variety of rocks, minerals, and geological environments are included in Keller (1987) and Palacky (1987).

The MT method can be used to probe the crust from depths of tens of meters to depths of tens of kilometers (Vozoff, 1991). Natural variations of the Earth's magnetic and electric field are measured and recorded at each MT station. The primary frequency bands used by the MT method are 10,000 to 1 hertz (Hz) from worldwide lightning activity and 1 to $0.0001 \mathrm{~Hz}$ from geomagnetic micro-pulsations. The natural electric and magnetic fields propagate vertically in the earth because the large resistivity contrast between the air and the earth causes a vertical refraction of both fields transmitted into the Earth (Vozoff, 1972).

The natural electric and magnetic fields are recorded in two orthogonal, horizontal directions. The vertical magnetic field ("tipper") also is recorded. The resulting time-series signals are used to derive the tensor apparent resistivities and phases. First, the signals are converted to complex cross-spectra using Fast-Fourier-Transform (FFT) techniques. Then, least-squares, cross-spectral analysis (Bendat and Piersol, 1971) is used to solve for a transfer function. Prior to conversion to apparent resistivity and phase, the tensor is normally rotated into principal directions that correspond to the direction of maximum and minimum apparent resistivity. For a two-dimensional (2-D) Earth, the MT fields can be decoupled into transverse electric (TE) and transverse magnetic (TM) modes; 2-D modeling generally is done to fit both modes. When the geology satisfies the 2-D assumption, the MT data for the TE mode are for the electric field parallel to geologic strike, and the data for the TM mode are for the electric field across strike. The MT method is well suited for studying complicated geological environments because the electric and magnetic relations are sensitive to vertical and horizontal variations in resistivity. The MT method is capable of establishing whether the electromagnetic fields are responding to subsurface terranes of effectively one, two, or threedimensions. An introduction to the MT method and references for a more advanced understanding are in Dobrin and Savit (1988) and Vozoff (1991).

\section{Magnetotelluric Survey}

Data were collected at 11 stations in 2004 to represent the area of this study. The station locations were chosen for constraining gravity modeling along the Pajarito Fault and for proximity to roads, and for avoidance of electrical noise such as power lines. All data at the stations were collected with a portable EMI MT-1 system (EMI, 1996). Horizontal electric fields 
were sensed using copper sulfate porous pots placed in an Lshaped, three-electrode array with dipole lengths of 30 meters

(m). The orthogonal, horizontal magnetic fields in the direction of the electric-field measurement array were sensed using permalloy-cored induction coils. Frequencies sampled ranged from 4 to $23,000 \mathrm{~Hz}(4.394,7.324,12.21,19.04,28.32,41.50,60.06$, $79.00,85.94,100.0,122.1,150.0,172.4,210.0,270.0,340.0$, $460.0,580.0,720.0,885.0,1170,1500,1870,2200,2730,3550$, $4900,6500,9000,11590,15290,19500,23370)$ and 0.009 to $70 \mathrm{~Hz}$ $(0.0088,0.0146,0.0244,0.0381,0.0566,0.0830,0.0879,0.1201$, $0.1465,0.1719,0.2441,0.3447,0.3809,0.5664,0.8301,1.201$, $1.758,2.441,2.920,3.447,4.883,7.617,11.328,16.602,24.023$, $34.375,48.828,68.945)$ using single-station recordings of the orthogonal, horizontal components of the electric and magnetic fields, and the vertical magnetic field.

The following table lists the $11 \mathrm{MT}$ station locations recorded using a global positioning system during field acquisition. Coordinates are referenced to the 1866 Clarke spheroid and North American 1927 Western United States datum. Longitude and latitude format is degrees:minutes:seconds. Universal Transverse Mercator (UTM) units are in meters. Station elevation is given in meters. The accuracy of the $x, y, z$ component is about \pm 5 meters.

\begin{tabular}{|c|c|c|c|c|c|}
\hline Station & Longitude & $\underline{\text { Latitude }}$ & $\begin{array}{c}\text { UTM } \\
\text { North }(\mathrm{m}) \\
\end{array}$ & $\begin{array}{c}\text { UTM } \\
\text { East }(\mathrm{m})\end{array}$ & Elevation (m) \\
\hline $35 \mathrm{~m}$ & $-106: 28: 31$ & $35: 49: 35$ & $3,965,476$ & $13,366,739$ & 2776 \\
\hline $34 \mathrm{~m}$ & $-106: 26: 18$ & $35: 49: 38$ & $3,965,540$ & $13,370,093$ & 2709 \\
\hline $33 m$ & $-106: 25: 26$ & $35: 47: 56$ & $3,962,360$ & $13,371,350$ & 2632 \\
\hline $32 \mathrm{~m}$ & $-106: 24: 45$ & $35: 47: 02$ & $3,960,693$ & $13,372,338$ & 2538 \\
\hline $31 \mathrm{~m}$ & $-106: 24: 37$ & $35: 43: 50$ & $3,954,777$ & $13,372,461$ & 2273 \\
\hline $30 \mathrm{~m}$ & $-106: 23: 37$ & $35: 42: 52$ & $3,952,970$ & $13,373,947$ & 2093 \\
\hline $36 \mathrm{~m}$ & $-106: 23: 00$ & $35: 42: 40$ & $3,952,577$ & $13,374,863$ & 2008 \\
\hline 15 & $-106: 13: 43$ & $35: 44: 37$ & $3,956,023$ & $13,388,902$ & 2047 \\
\hline $28 \mathrm{~m}$ & $-106: 13: 38$ & $35: 42: 09$ & $3,951,444$ & $13,388,978$ & 2037 \\
\hline 14 & $-106: 13: 31$ & $35: 40: 20$ & $3,948,096$ & $13,389,104$ & 2100 \\
\hline $29 \mathrm{~m}$ & $-106: 13: 34$ & $35: 38: 09$ & $3,944,059$ & $13,388,978$ & 1989 \\
\hline
\end{tabular}

\section{Magnetotelluric Data}

The recorded time-series data were transformed to the frequency domain and processed to determine a 2-D apparent resistivity and phase tensor at each site. Rotation of the impedance tensor to maximum and minimum directions allows for decoupling into the TE and TM modes. All data were rotated to 47 degrees.

Although true remote reference techniques were not used in the survey, cross-power files were sorted to select optimal signal-to-noise time-series data sets (see Appendix at the back of the report). 
The effects of near-surface resistivity anomalies caused "static shifts" in the data (Sternberg and others, 1988). Static shifts were significant in the MT stations 32 and 29, although the static shift is resolved in the AMT data for station 32. cultural features can affect the response of the MT system. Fences, pipelines, communication lines, railways, and other manmade conductors can contaminate the responses.

The figures in the Appendix represent the field-processed MT data for each station after the time-series data were converted to the frequency domain, and the tensor-transfer function was rotated into principal directions as described in the "Magnetotelluric Method" section. For each station, eight separate plots are given:

1. Apparent Resistivity for the rotated maximum (x symbol) and minimum (o symbol) modes

2. Impedance Phase for the rotated maximum ( $x$ symbol) and minimum (o symbol) modes

3. Impedance Skew

4. Multiple Coherency for the rotated maximum (x symbol) and minimum (o symbol) modes

5. Impedance Polar Plots

6. Tipper Magnitude

7. Tipper Strike

8. $\mathrm{HzHx}$ (x symbol) and HzHy (o symbol) Coherency

Error bars (], [) on the Apparent Resistivity, Impedance Phase, Skew, Tipper Magnitude, and Tipper Strike plots represent probable errors within one standard deviation of the sample variance (Gamble and others, 1979).

Apparent resistivity is the ratio of the electric-field strength magnitude over the magnetic-field strength magnitude for a given frequency. The impedance phase is proportional to the slope of the apparent resistivity curve on a log-log plot, but from a baseline at -45 degrees (Vozoff, 1991). A measure of the dimensionality for MT data is provided by the impedance skew of the impedance tensor (Vozoff, 1972). If the effective, measured resistivity response to the geology beneath a MT station is truly one or two dimensional, then the skew will be zero. Both instrument and environmental sources of noise contribute to nonzero skew values but are typically small (about 0.1) for relatively low-noise-level recordings. Higher skews (above 0.2) are an indication of either the resistivity response to 3-D geology or higher levels of noise. Manmade electrical noise, such as power lines, power generators, and moving vehicles and trains, can have a negative effect on MT data quality. All of these local disturbances produce an incoherent noise mainly affecting frequencies above $1 \mathrm{~Hz}$. Other manmade electrical noise, such as direct current electric trains and active cathodic 
protection of pipelines, produce coherent electromagnetic signals mainly affecting frequencies below $1 \mathrm{~Hz}$.

In the survey area, noise from a number of small power lines and small moving vehicles was negligible at distances of $0.4 \mathrm{~km}$ and greater from the noise source. Power-line signal levels were measured at each site and were typically less than 20 percent of the maximum recordable signals. Noise from larger power lines, power generators, pipelines, and trains was negligible at distances greater than $5 \mathrm{~km}$. Local lightning, wind, and rainstorms also can degrade data quality, but these were avoided by not recording during active thunderstorm periods. Burying the magnetic induction coils and keeping the electric dipole wires flat on the ground surface minimized wind noise.

Predicted values of the electric field can be computed from the measured values of the magnetic field (Vozoff, 1991). The coherence of the predicted electric field with the measured electric field is a measure of the signal-to-noise ratio provided in the multiple coherency plots. Values are normalized between 0 and 1 , where values at 0.5 signify signal levels equal to noise levels. For this data set, coherencies were generally at an acceptable level, except at times in the "dead band" frequency ranges $(0.01$ to $5 \mathrm{~Hz}$ and 1,000 to $5,000 \mathrm{~Hz})$.

The figures in the Appendix represent the field-processed MT data at each station, which includes some data scatter and poor signal-to-noise ratios. The only effort at removing noisy data points was to visually inspect and select the best signal-tonoise field data to combine into the final data plots.

The impedance polar plots provide a measure of the MT data dimensionality (Reddy and others, 1977). For 1-D resistivity structures, the principal impedance polar diagram (dashed line) is a circle. For 2-D or 3-D resistivity structures, the principal impedance polar diagram (dashed line) elongates either parallel or perpendicular to strike direction. Over resistors, the principal impedance polar diagram elongates perpendicular to strike direction, and over conductors, the principal impedance polar diagram elongates parallel to strike direction. For 2-D resistivity structures, the additional impedance polar diagram (solid line) attains the shape of a symmetric clover leaf. For 3-D resistivity structures, the additional impedance polar diagram (solid line) elongates in one direction, and its amplitude is comparable to that of the principal impedance polar diagram (dashed line). Station 35 indicates a minor 3-D response over all of the frequencies recorded. Stations 34 and 36 indicate a 3-D response below $0.05 \mathrm{~Hz}$. Stations 30 and 33 indicate a 3-D response below $0.172 \mathrm{~Hz}$. Stations 31 and 32 indicate a 3-D response below $0.1 \mathrm{~Hz}$.

The tipper can be calculated when the vertical component of the magnetic field is measured. The tipper magnitude is a measure of the tipping of the magnetic field out of the horizontal plane (Vozoff, 1991). The magnitude is zero for the 
1-D case and typically increases between 0.1 to 0.5 , and rarely as great as 1, as it responds to vertical and sub-vertical structures. The tipper strike typically is used to help resolve the 90-degree ambiguity in the impedance rotation angle. The tipper magnitude of these stations typically was 0.1 to 0.6 over the lower frequencies, indicating some vertical structure at depth. The HzHx and HzHy coherency is a measure of the signalto-noise ratio of the vertical magnetic field with respect to each of the orthogonal, horizontal magnetic-field directions. Values are normalized between 0 and 1 , where values at 0.5 signify signal levels equal to noise levels. These threecomponent magnetic-field coherencies provide a check on the signal-to-noise ratio of the measured values in the tipper magnitude and tipper strike plots.

\section{References Cited}

Bendat, J.S., and Piersol, A.G., 1971, Random data-analysis and measurement procedures: New York, Wiley Interscience, $407 p$.

Dobrin, M.D., and Savit, C.H., 1988, Introduction to geophysical prospecting (4th ed.): New York, McGraw-Hill, $867 \mathrm{p}$.

Eberhart-Phillips, Donna, Stanley, W.D., Rodriguez, B.D., and Lutter, W.J., 1995, Surface seismic and electrical methods to detect fluids related to faulting: Journal of Geophysical Research, v. 100, no. B7, p.12919-12936.

EMI, 1996, MT-1 magnetotelluric system operation manual, version 3.2: Richmond, California, ElectroMagnetic Instruments, Inc., 220p.

Gamble, T.D., Goubau, W.M., and Clarke, J., 1979, Error analysis for remote reference magnetotellurics: Geophysics, v. 44, no. 5, p. 959-968.

Keller, G.V., 1987, Rock and mineral properties, in Nabighian, M.N., Ed., Electromagnetic methods in applied geophysics theory: Tulsa, Oklahoma, Society of Exploration Geophysicists, v. 1, p. 13-51. 
Keller, G.V., 1989, Electrical properties, in Carmichael, R.S., ed., Practical handbook of physical properties of rocks and minerals: Boca Raton, Florida, CRC Press, p. 359-427.

Nelson, P.H. and Anderson, L.A., 1992, Physical properties of ash flow tuff from Yucca Mountain, Nevada: Journal of Geophysical Research, vol. 97, no. B5, p. 6823-6841.

Palacky, G.J., 1987, Resistivity characteristics of geologic targets, in Nabighian, M.N., ed., Electromagnetic methods in applied geophysics theory: Tulsa, Oklahoma, Society of Exploration Geophysicists, vol. 1, p. 53-129.

Reddy, I.K., Rankin, D., and Phillips, R.J., 1977, ThreeDimensional modelling in magnetotelluric and magnetic variational sounding: Geophysics Journal of the Royal Astronomical Society, vol. 51, p. 313-325.

Sternberg, B.K., Washburne, J.C., and Pellerin, Louise, 1988, Correction for the static shift in magnetotellurics using transient electromagnetic soundings: Geophysics, vol. 53, p. 1459-1468.

Vozoff, Keeva, 1972, The magnetotelluric method in the exploration of sedimentary basins: Geophysics, vol. 37, p. $98-141$.

Vozoff, Keeva, 1991, The magnetotelluric method, in Nabighian, M.N., ed., Electromagnetic methods in applied geophysics: Tulsa, Oklahoma, Society of Exploration Geophysicists, v. 2, pt. B, p. 641-711. 


\section{APPENDIX MAGNETOTELLURIC DATA PLOTS}

There are eight separate plots for each station:

1. Apparent Resistivity for the rotated maximum ( $x$ symbol) and minimum (o symbol) modes

2. Impedance Phase for the rotated maximum ( $x$ symbol) and minimum (o symbol) modes

3. Impedance Skew for the impedance tensor

4. Multiple Coherency for the rotated maximum ( $x$ symbol) and minimum (o symbol) modes of the electric field

5. Impedance Polar Plots (at 12 selected frequencies)

6. Tipper Magnitude for the vertical magnetic field

7. Tipper Strike for the vertical magnetic field

8. $\mathrm{HzHx}$ (x symbol) and HzHy (o symbol) Coherency

Refer to the "Magnetotelluric Data" section in this report for an explanation of these plots. 


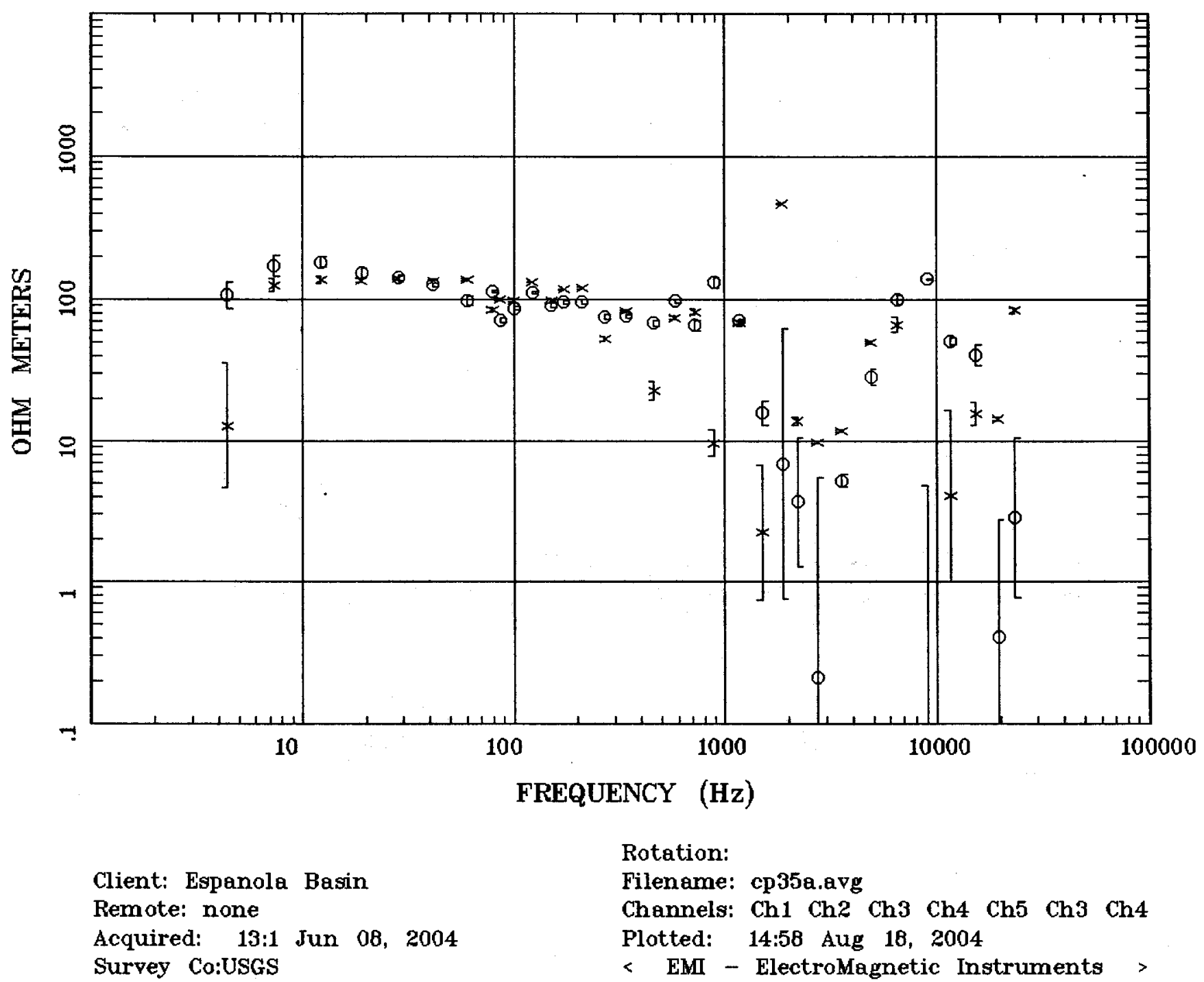




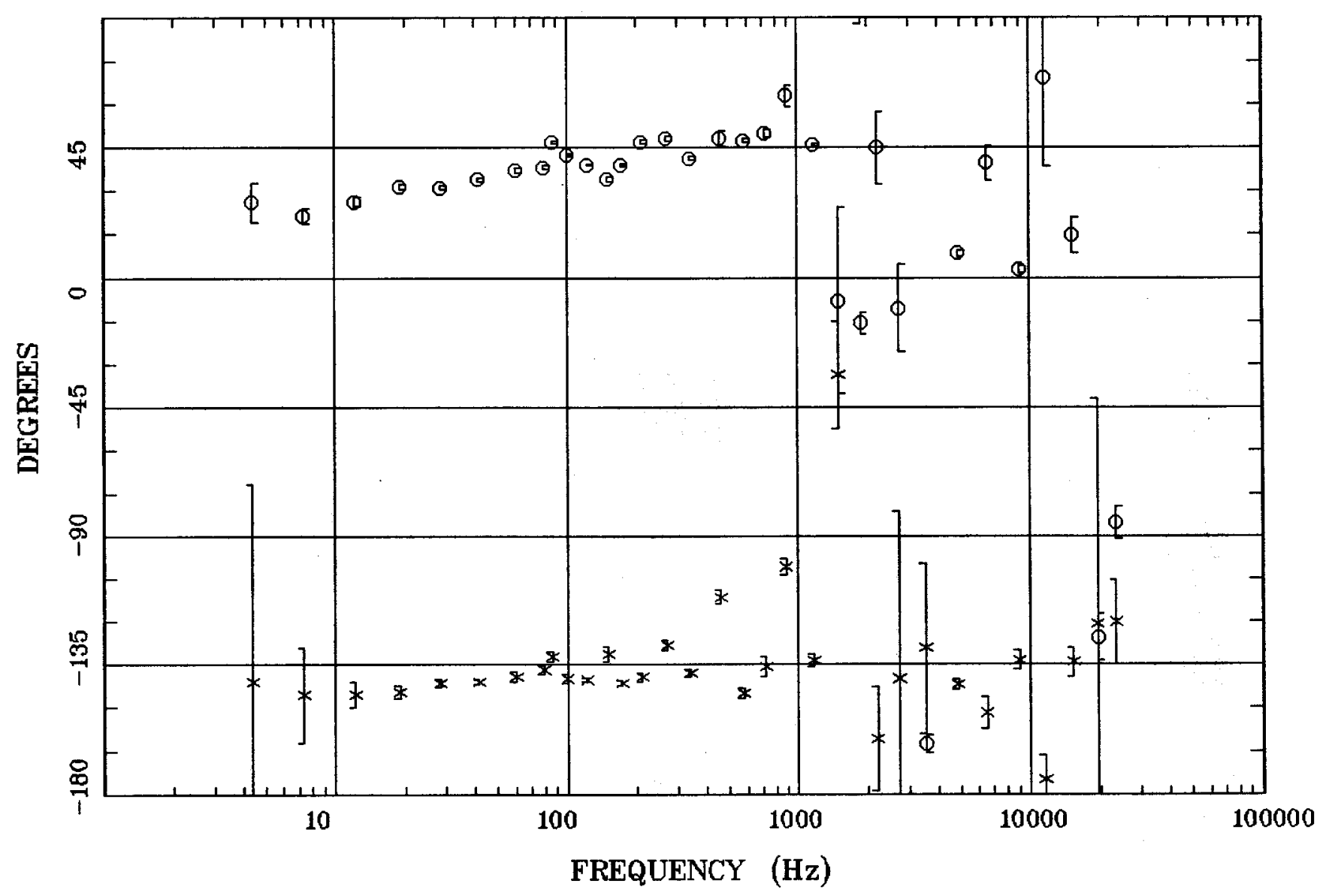

Client: Espanola Basin Remote: none Acquired: 13:1 Jun 08, 2004 Survey Co:USGS
Rotation:

Filename: cp35a.avg

Channels: Ch1 Ch2 Ch3 Ch4 Ch5 Ch3 Ch4 Plotted: 14:58 Aug 18, 2004

< EMI - ElectroMagnetic Instruments > 


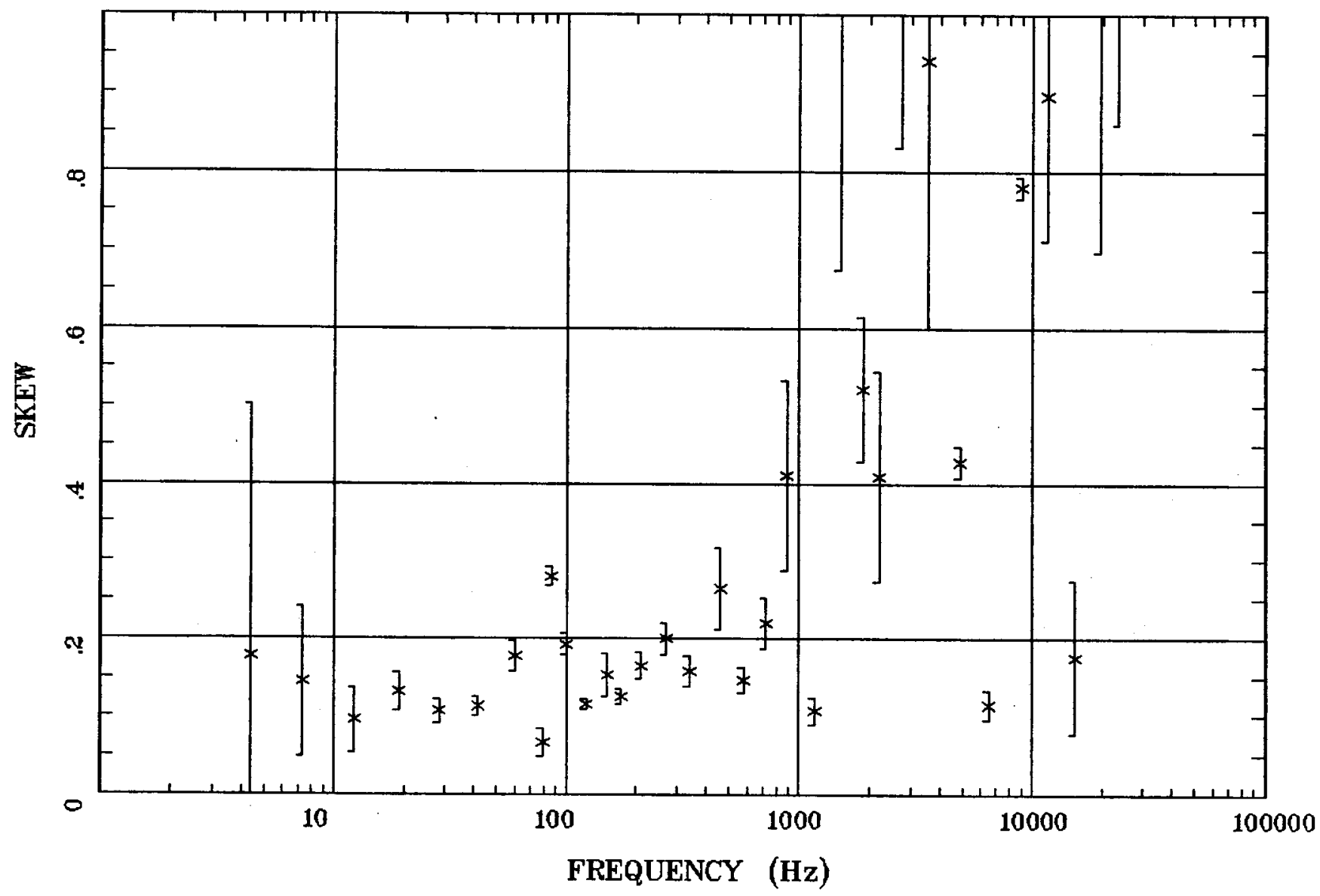

Client: Espanola Basin

Remote: none

Acquired: 13:1 Jun 08, 2004

Survey Co:USGS
Rotation:

Filename: cp35a.avg

Channels: Ch1 Ch2 Ch3 Ch4 Ch5 Ch3 Ch4

Plotted: 14:58 Aug 18, 2004

< EMI - ElectroMagnetic Instruments > 


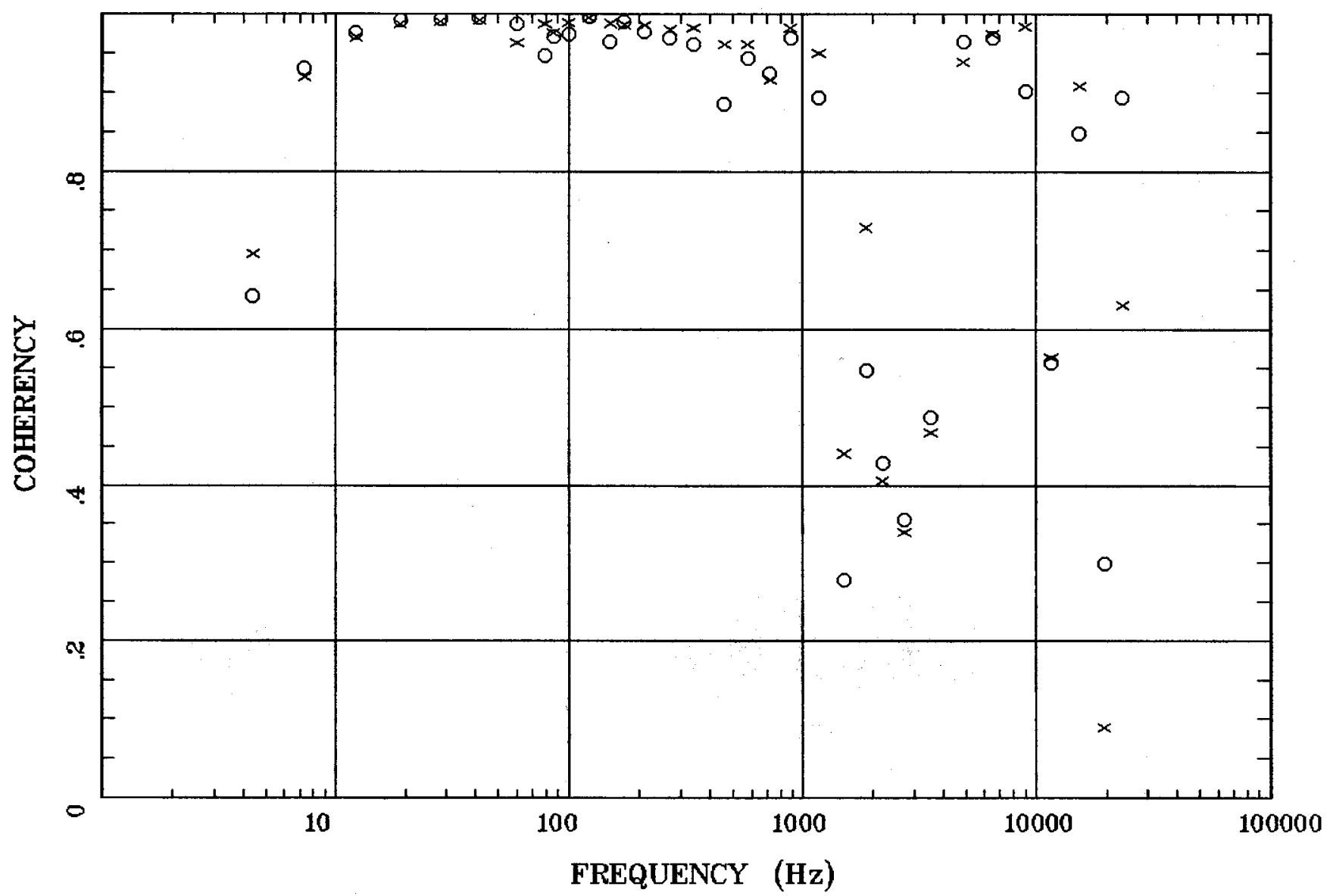

Client: Espanola Basin

Remote: none

Acquired: 13:1 Jun 08, 2004 Survey Co:USGS
Rotation:

Filename: cp35a.avg

Channels: Ch1 Ch2 Ch3 Ch4 Ch5 Ch3 Ch4 Plotted: 14:58 Aug 18, 2004

< EMI - ElectroMagnetic Instruments > 


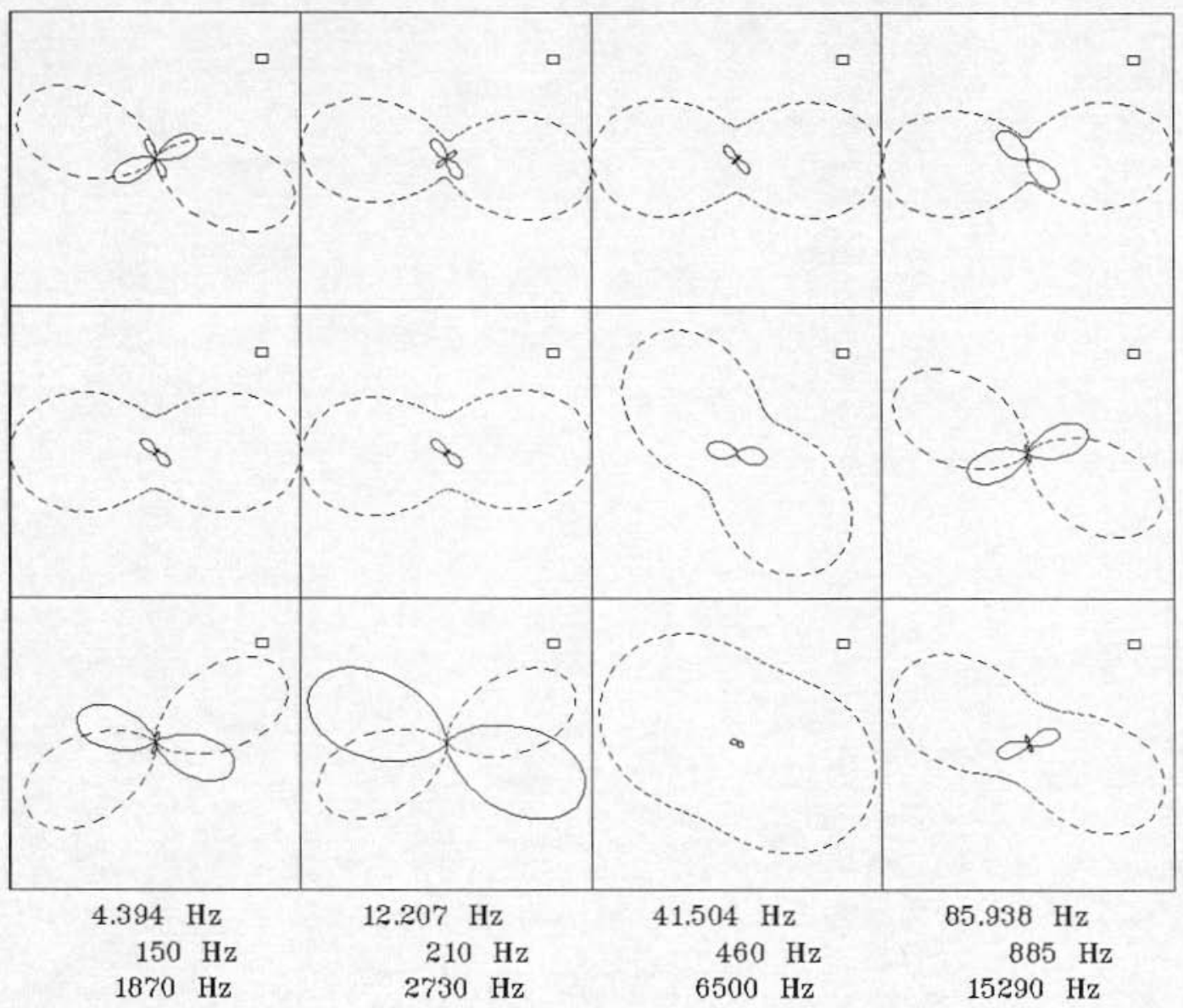

Client: Espanola Basin

Remote: none

Acquired: 13:1 Jun 08, 2004

Survey Co:USGS
Rotation:

Filename: cp35a.avg

Channels: Ch1 Ch2 Ch3 Ch4 Ch5 Ch3 Ch4 Plotted: 14:58 Aug 18, 2004

$<$ EMI - ElectroMagnetic Instruments 


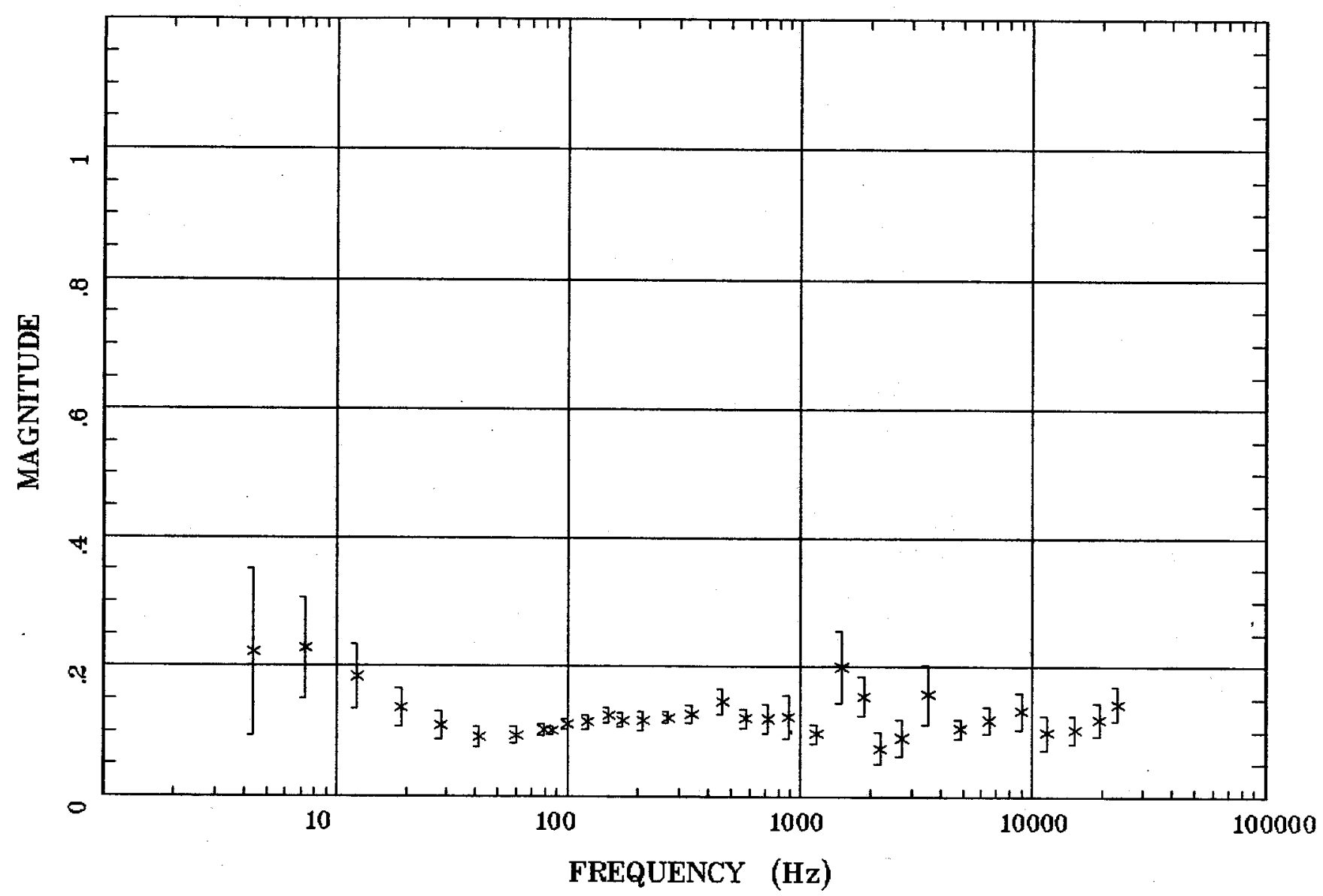

Client: Espanola Basin Remote: none Acquired: 13:1 Jun 08, 2004 Survey Co:USGS
Rotation:

Filename: cp35a.avg

Channels: Ch1 Ch2 Ch3 Ch4 Ch5 Ch3 Ch4

Plotted: 14:58 Aug 18, 2004

< EMI - ElectroMagnetic Instruments 


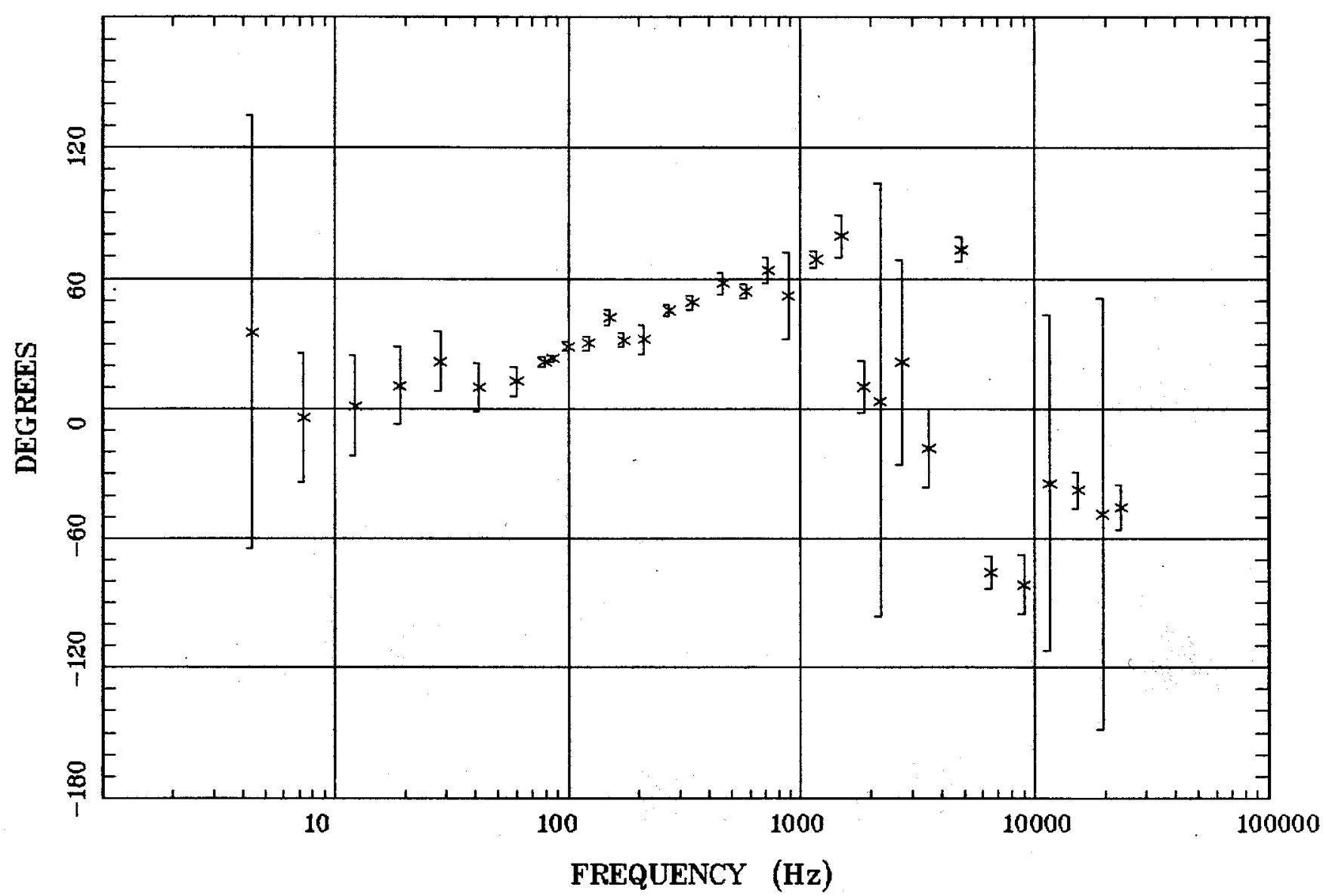

Client: Espanola Basin Remote: none

Acquired: 13:1 Jun 08, 2004 Survey Co:USGS
Rotation:

Filename: cp35a.avg

Channels: Ch1 Ch2 Ch3 Ch4 Ch5 Ch3 Ch4 Plotted: 14:58 Aug 18, 2004

< EMI - ElectroMagnetic Instruments > 
HzHx.x Coh HzHy.o

Los Alamos, NM 100k

Station 35

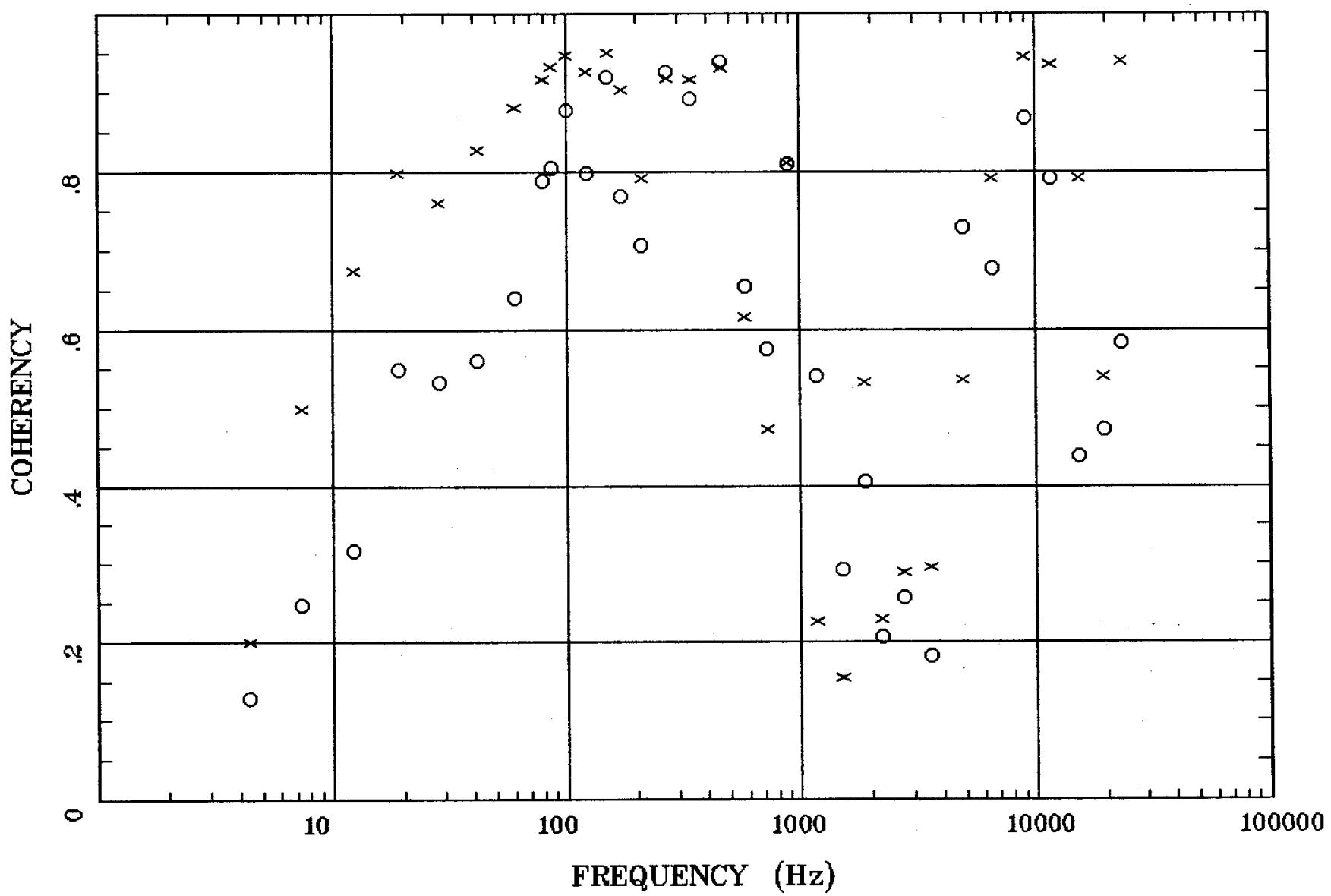

Client: Espanala Basin.

Remote: none

Acquired: 13:1 Jun 08, 2004 Survey Co:USGS
Rotation:

Filename: cp35a.avg

Channels: Ch1 Ch2 Ch3 Ch4 Ch5 Ch3 Ch4

Plotted: 14:58 Aug 18, 2004

< EMI - ElectroMagnetic Instruments 


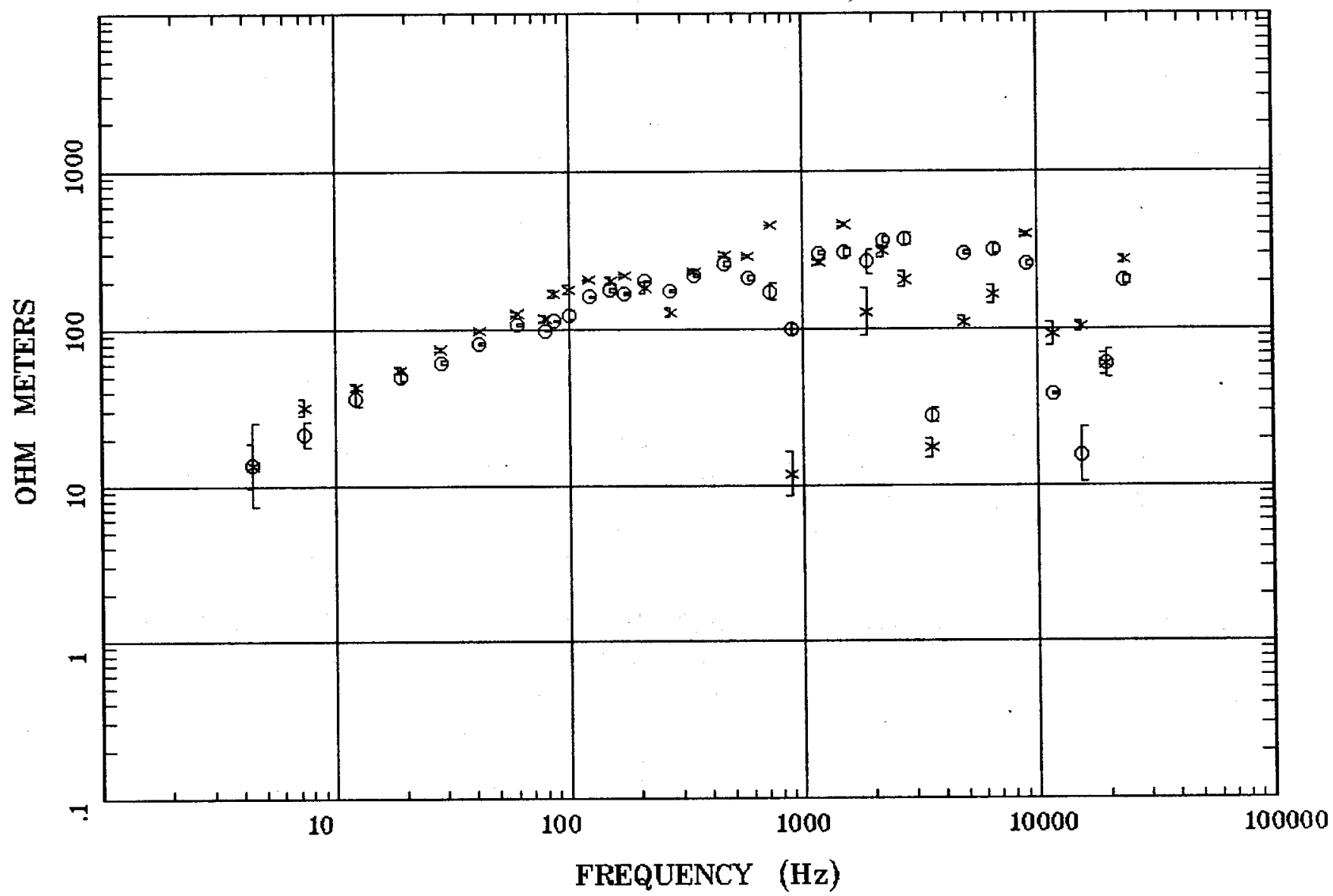

Client: Espanola Basin Remote: none Acquired: 13:0 Jul 16, 2004 Survey Co:USGS
Rotation:

Filename: cp34a.avg

Channels: Ch1 Ch2 Ch3 Ch4 Ch5 Ch3 Ch4 Plotted: 15:00 Aug 18, 2004

< EMI - ElectroMagnetic Instruments > 
Station 34

IMPEDANCE PHASE

Los Alamos, NM 100k

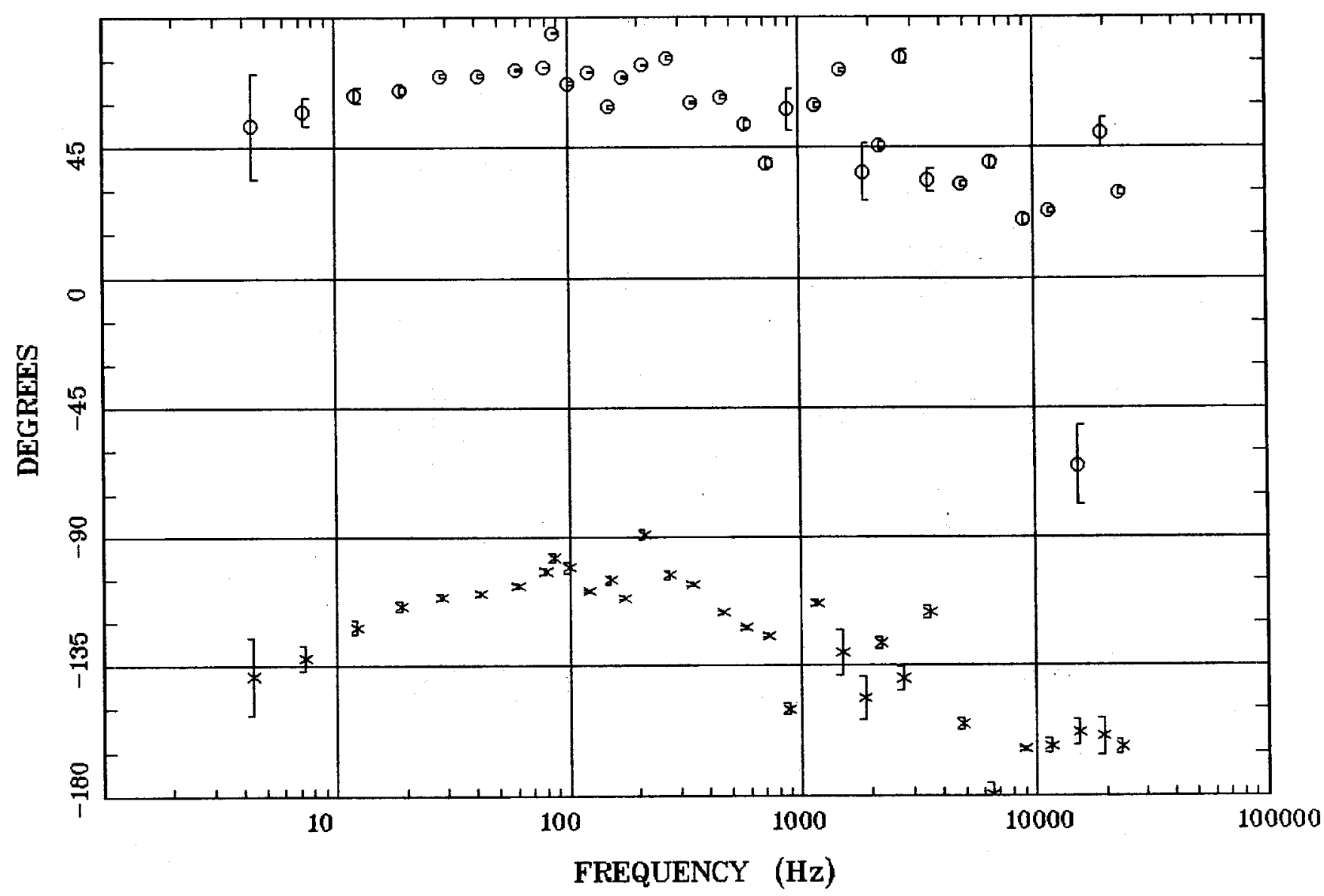

Client: Espanola Basin Remote: none Acquired: 13:0 Jul 16, 2004 Survey Co:USGS
Rotation:

Filename: cp34a.avg

Channels: Ch1 Ch2 Ch3 Ch4 Ch5 Ch3 Ch4 Plotted: 15:00 Aug 18, 2004

< EMI - ElectroMagnetic Instruments > 
Los Alamos, NM 100k

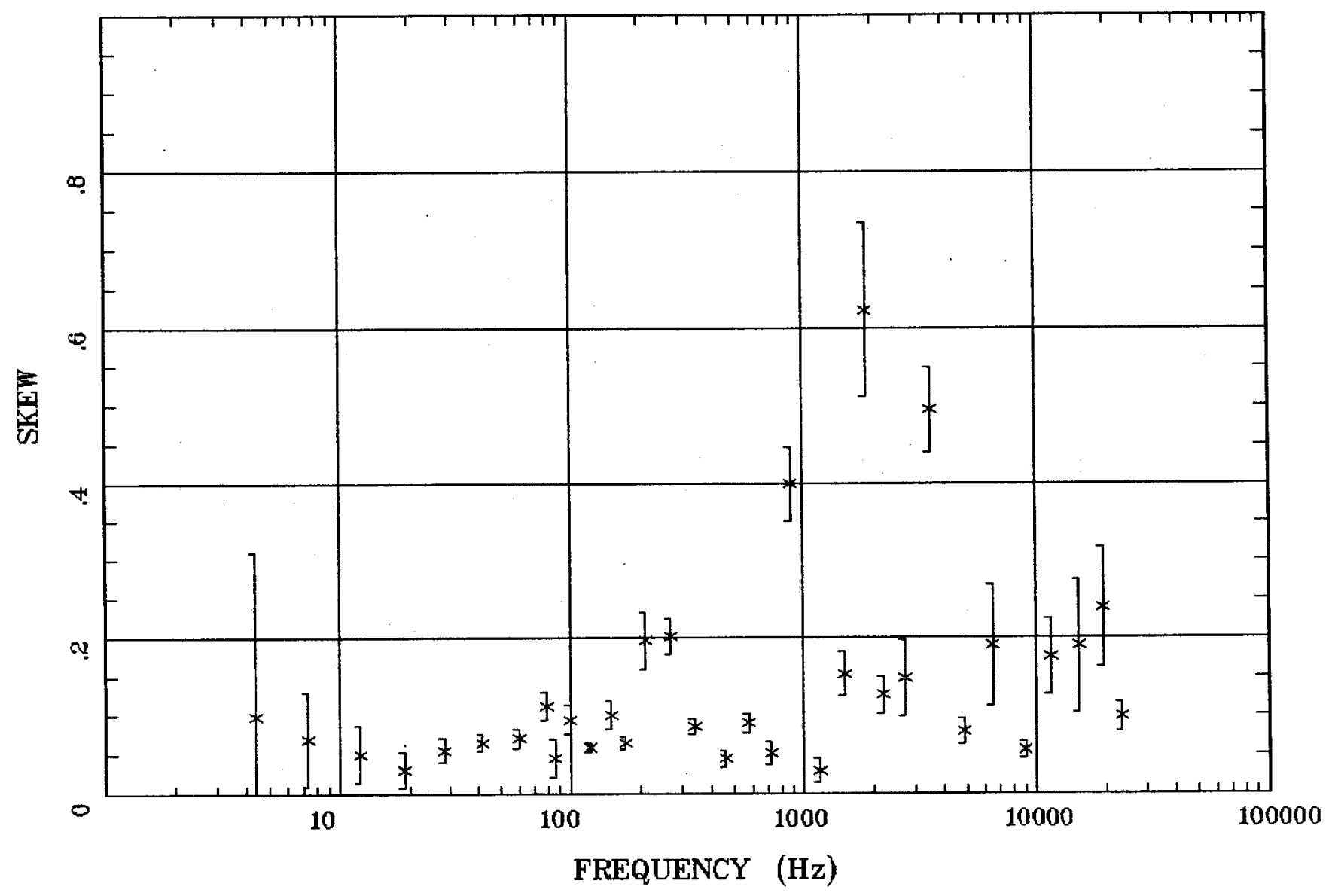

Client: Espanola Basin Remote: none Acquired: 13:0 Jul 16, 2004 Survey Co:USGS
Rotation:

Filename: cp34a.avg

Channels: Ch1 Ch2 Ch3 Ch4 Ch5 Ch3 Ch4 Platted: 15:00 Aug 18, 2004

< EMI - ElectroMagnetic Instruments > 
E MULT Coh.

Los Alamos, NM 100k

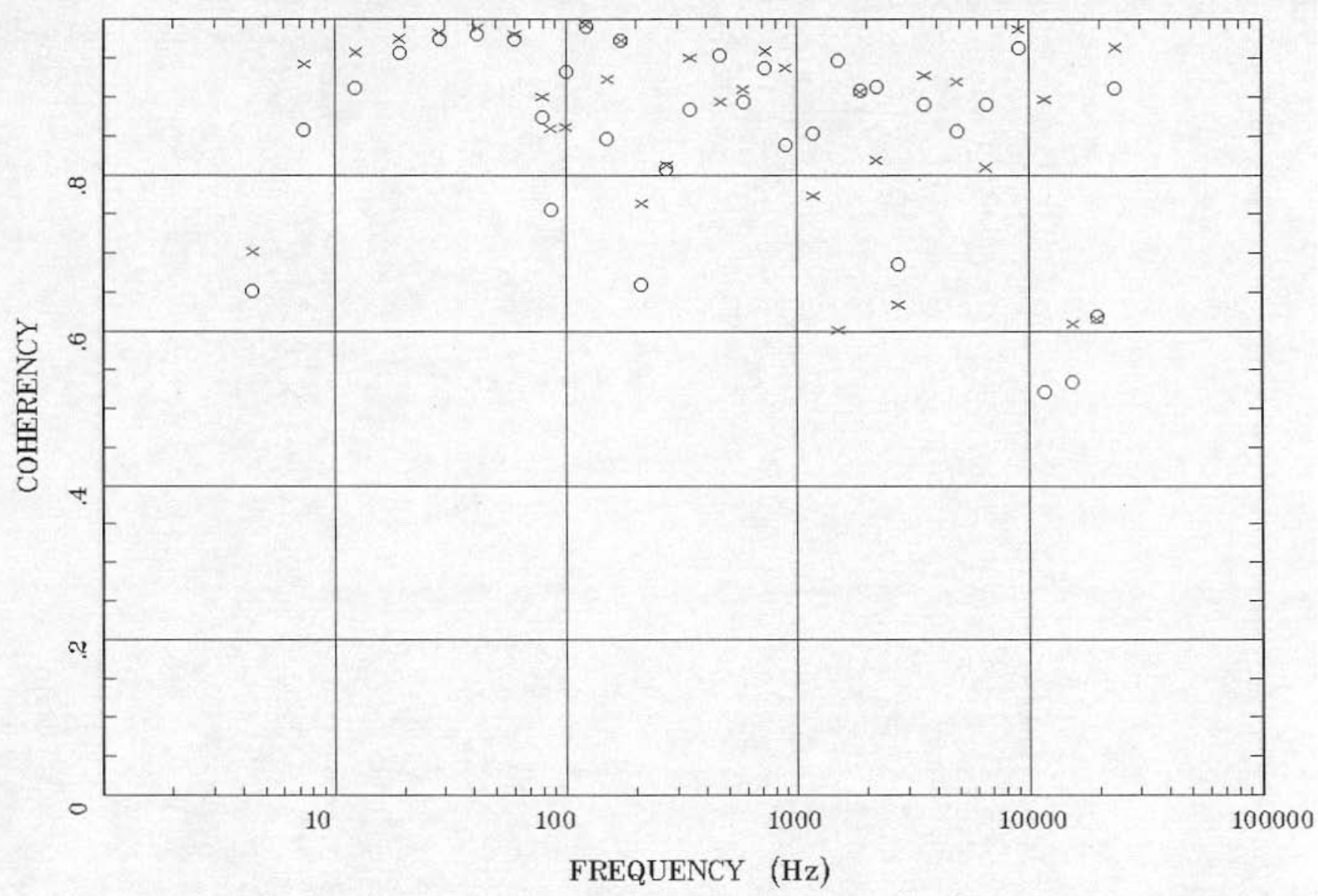

Client: Espanola Basin

Remote: none

Acquired: 13:0 Jul 16, 2004

Survey Co:USGS
Rotation:

Filename: cp34a.avg

Channels: Ch1 Ch2 Ch3 Ch4 Ch5 Ch3 Ch4

Plotted: 15:00 Aug 18, 2004

< EMI - ElectroMagnetic Instruments > 
Los Alamos, NM 100k

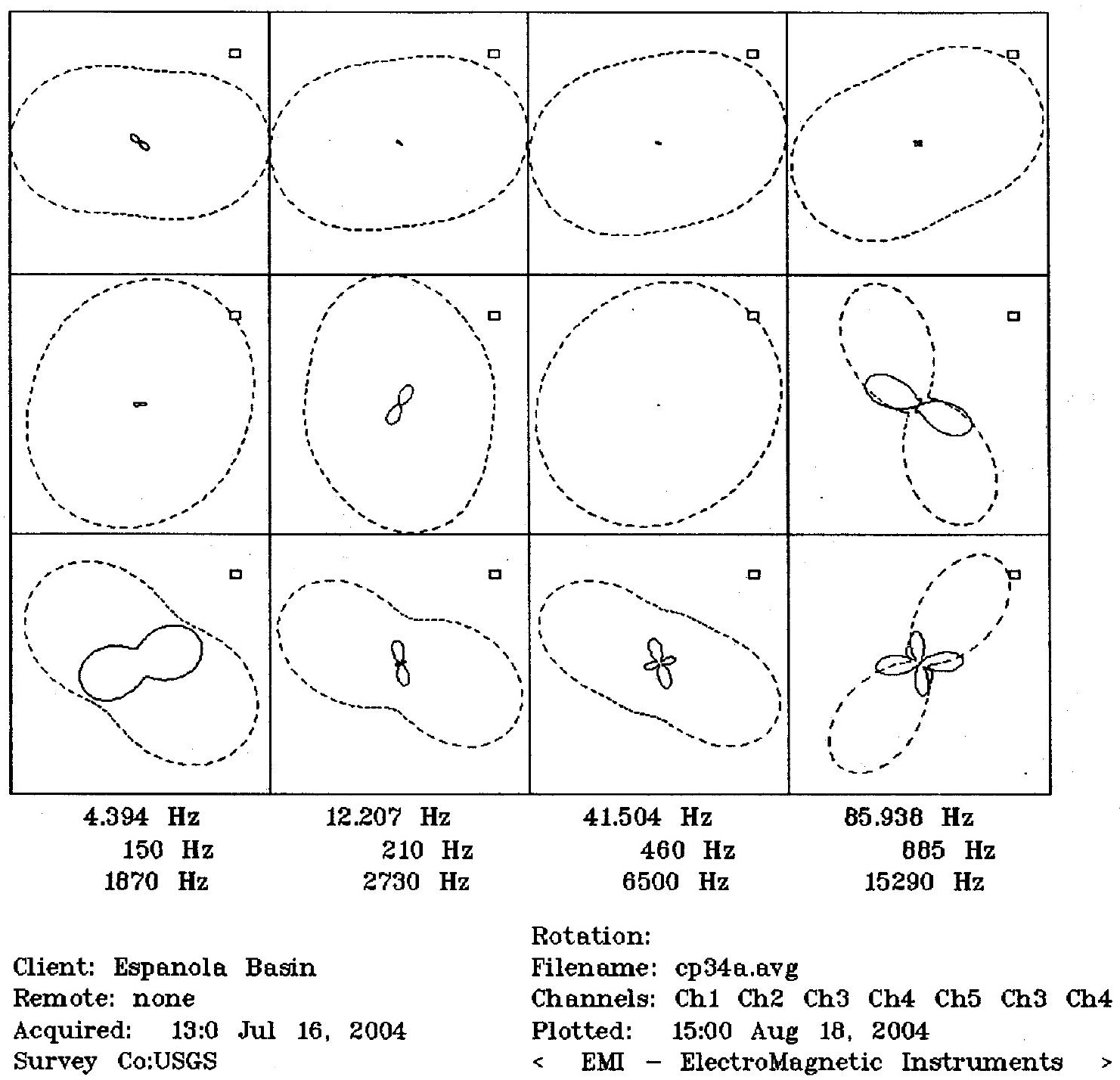


Station 34

TIPPER MAGNITUDE

Los Alamos, NM 100k

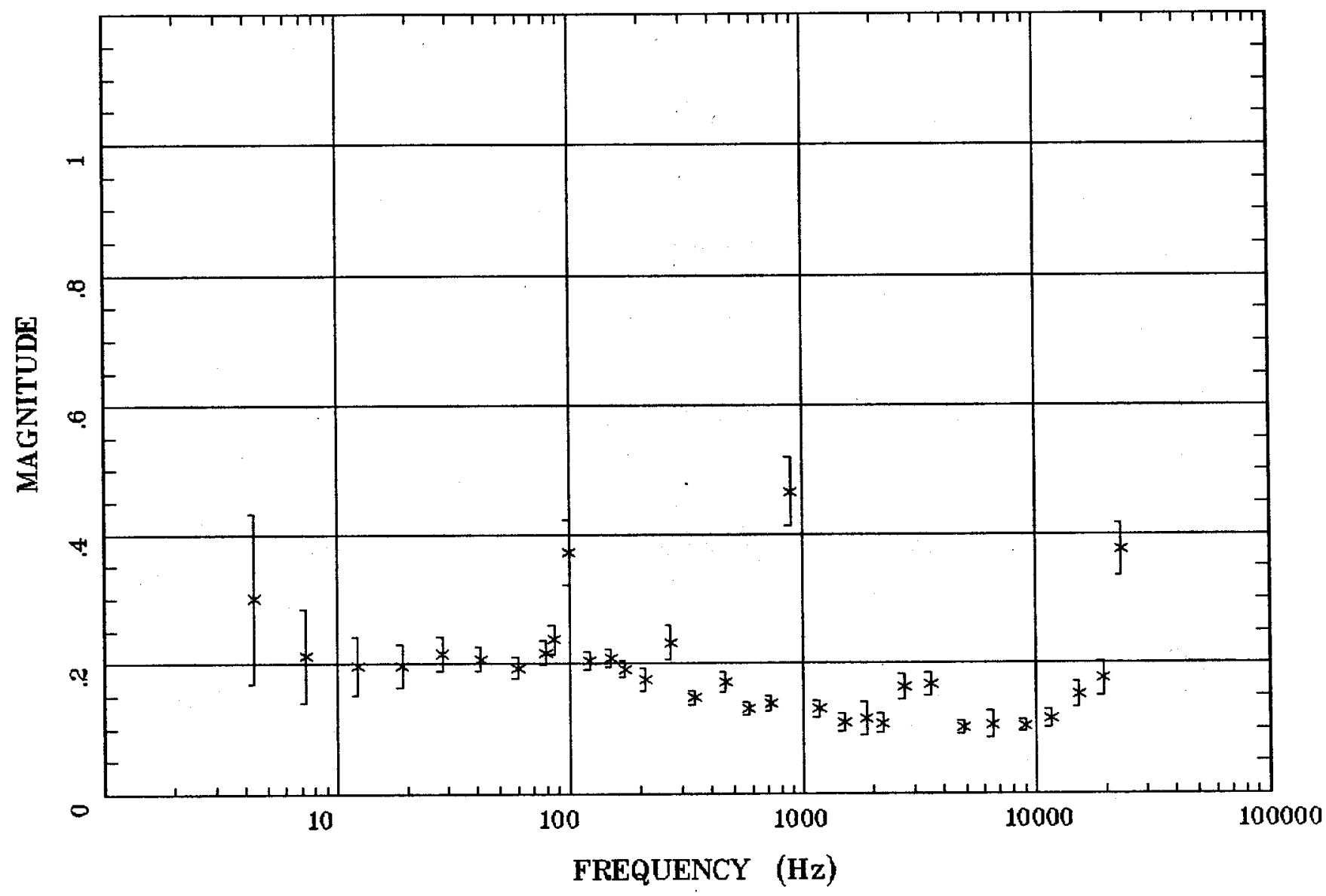

Client: Espanola Basin Remote: none

Acquired: 13:0 Jul 16, 2004 Survey Co:USGS
Rotation:

Filename: cp34a.avg

Channels: Ch1 Ch2 Ch3 Ch4 Ch5 Ch3 Ch4 Plotted: 15:00 Aug 18, 2004

< EMI - ElectroMagnetic Instruments > 


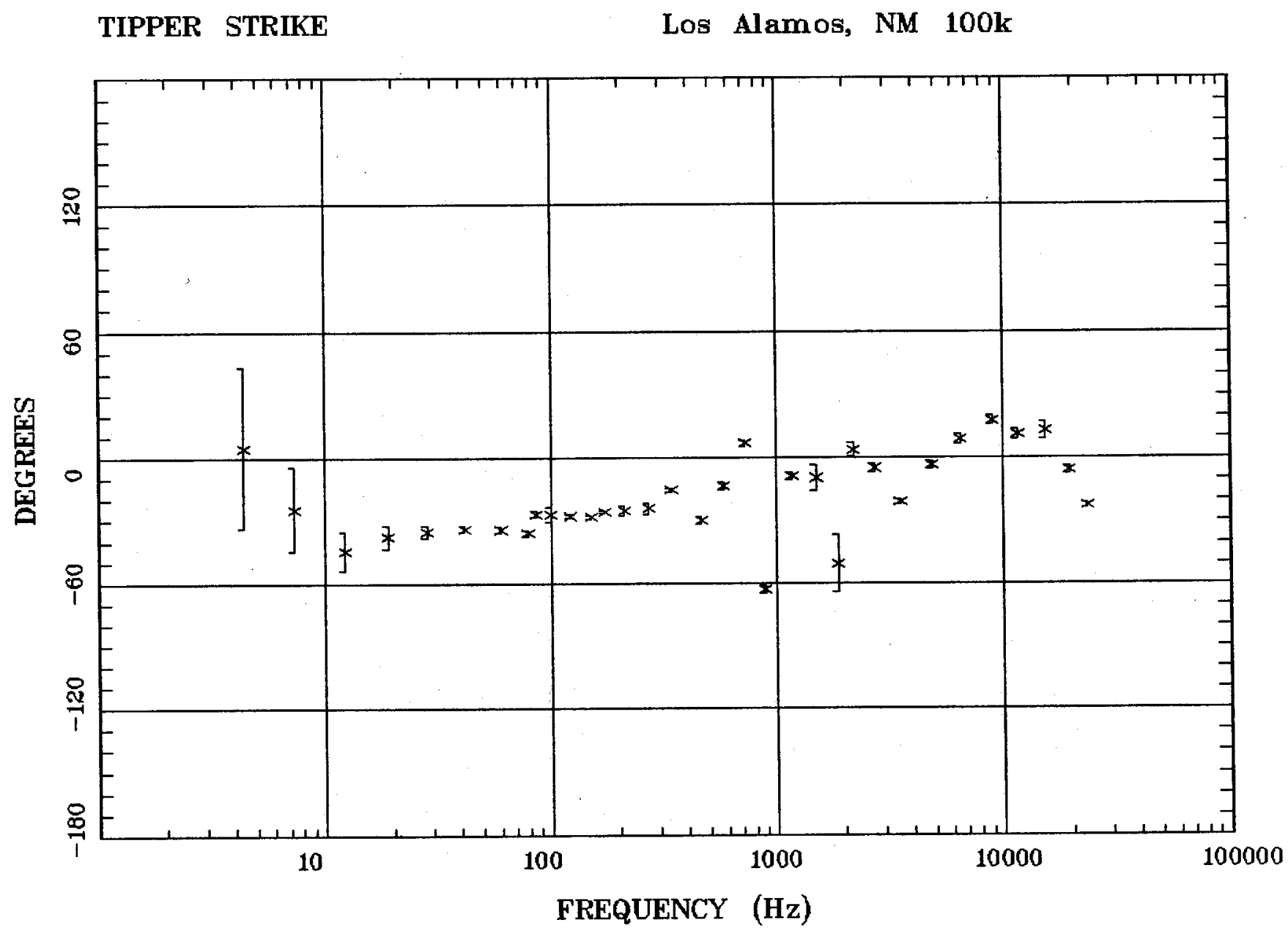

Client: Espanola Basin

Remote: none

Acquired: 13:0 Jul 16, 2004

Survey Co:USGS
Rotation:

Filename: cp34a.avg

Channels: Ch1 Ch2 Ch3 Ch4 Ch5 Ch3 Ch4

Plotted: 15:00 Aug 18, 2004

< EMI - ElectroMagnetic Instruments > 
HzHx.x Coh HzHy.o

Los Alamos, NM 100k

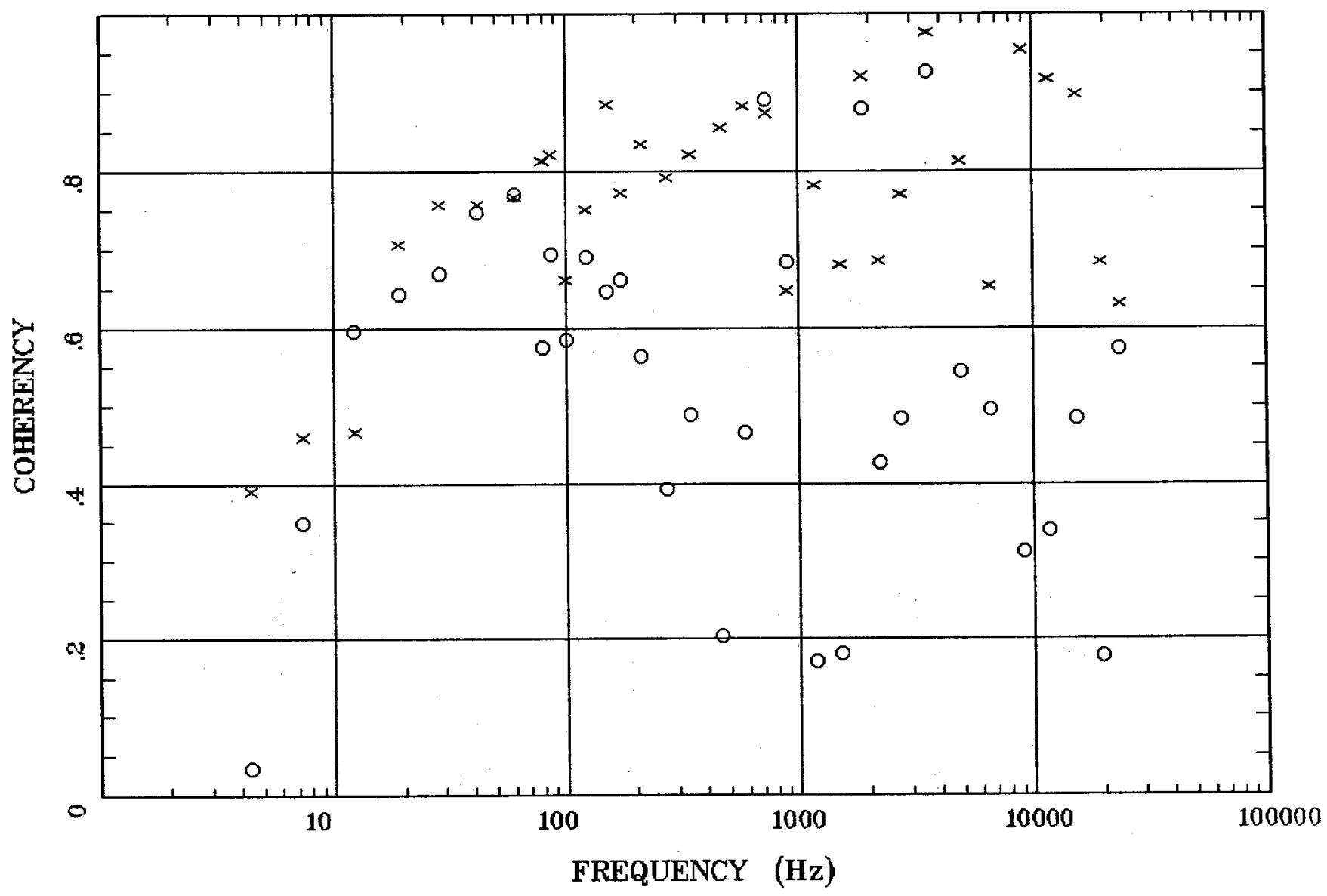

Client: Espanola Basin

Remote: none

Acquired: 13:0 Jul 16, 2004 Survey Co:USGS
Rotation:

Filename: cp34a.avg

Channels: Ch1 Ch2 Ch3 Ch4 Ch5 Ch3 Ch4 Plotted: 15:00 Aug 18, 2004

< EMI - ElectroMagnetic Instruments 


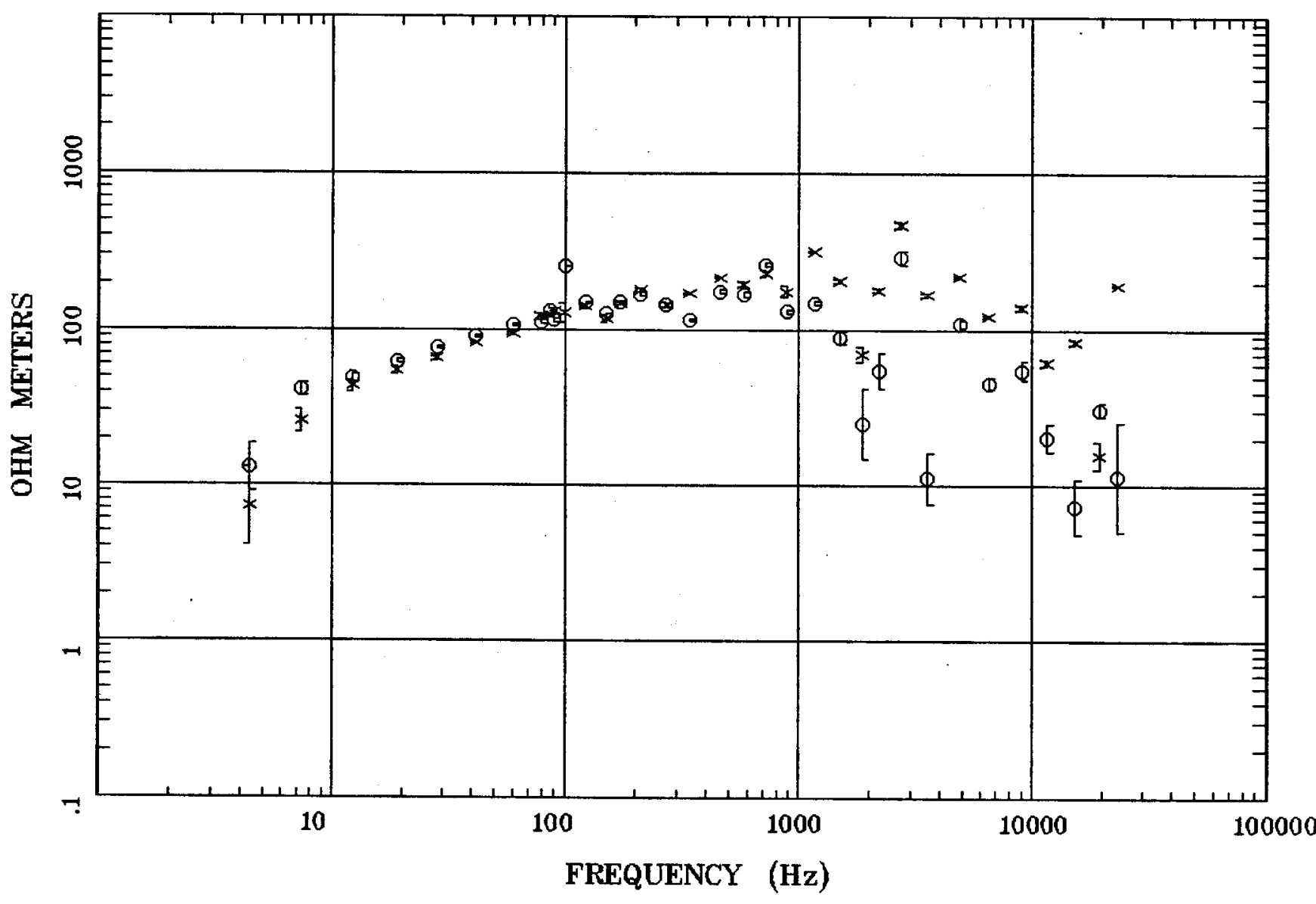

Client: Espanola Basin

Remote: none

Acquired: 16:0 Jul 13, 2004

Survey Co:USGS
Rotation:

Filename: cp33aall.avg

Channels: Ch1 Ch2 Ch3 Ch4 Ch5 Ch3 Ch4 Plotted: 15:01 Aug 18, 2004

$<$ EMI - ElectroMagnetic Instruments > 
IMPEDANCE PHASE

Los Alamos, NM 100k

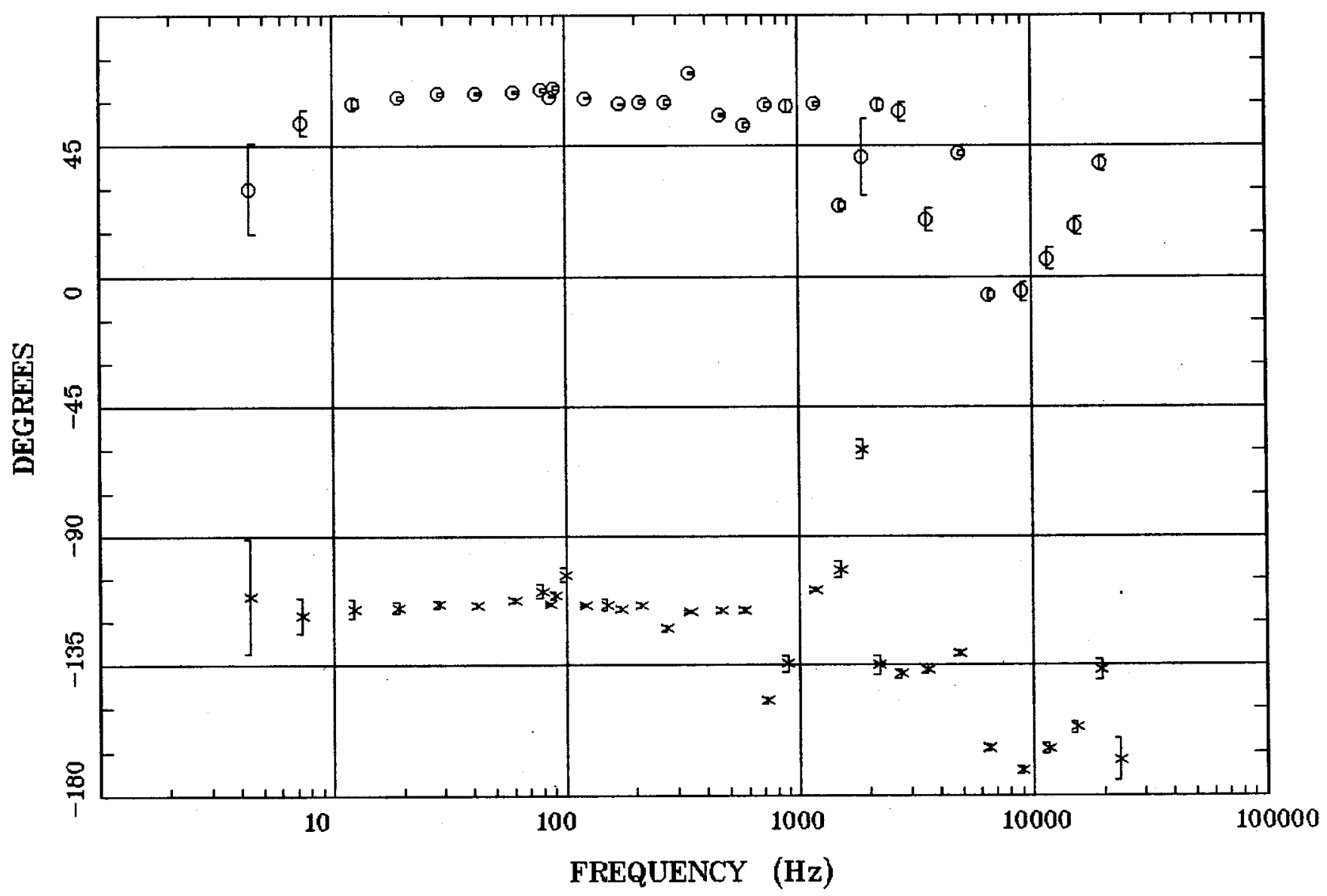

Client: Espanola Basin

Remote: none

Acquired: 16:0 Jul 13, 2004

Survey Co:USGS
Rotation:

Filename: cp33aall.avg

Channels: Ch1 Ch2 Ch3 Ch4 Ch5 Ch3 Ch4

Plotted: 15:01 Aug 18, 2004

< EMI - ElectroMagnetic Instruments > 
Station 33

IMPEDANCE SKEW

Los Alamos, NM 100k

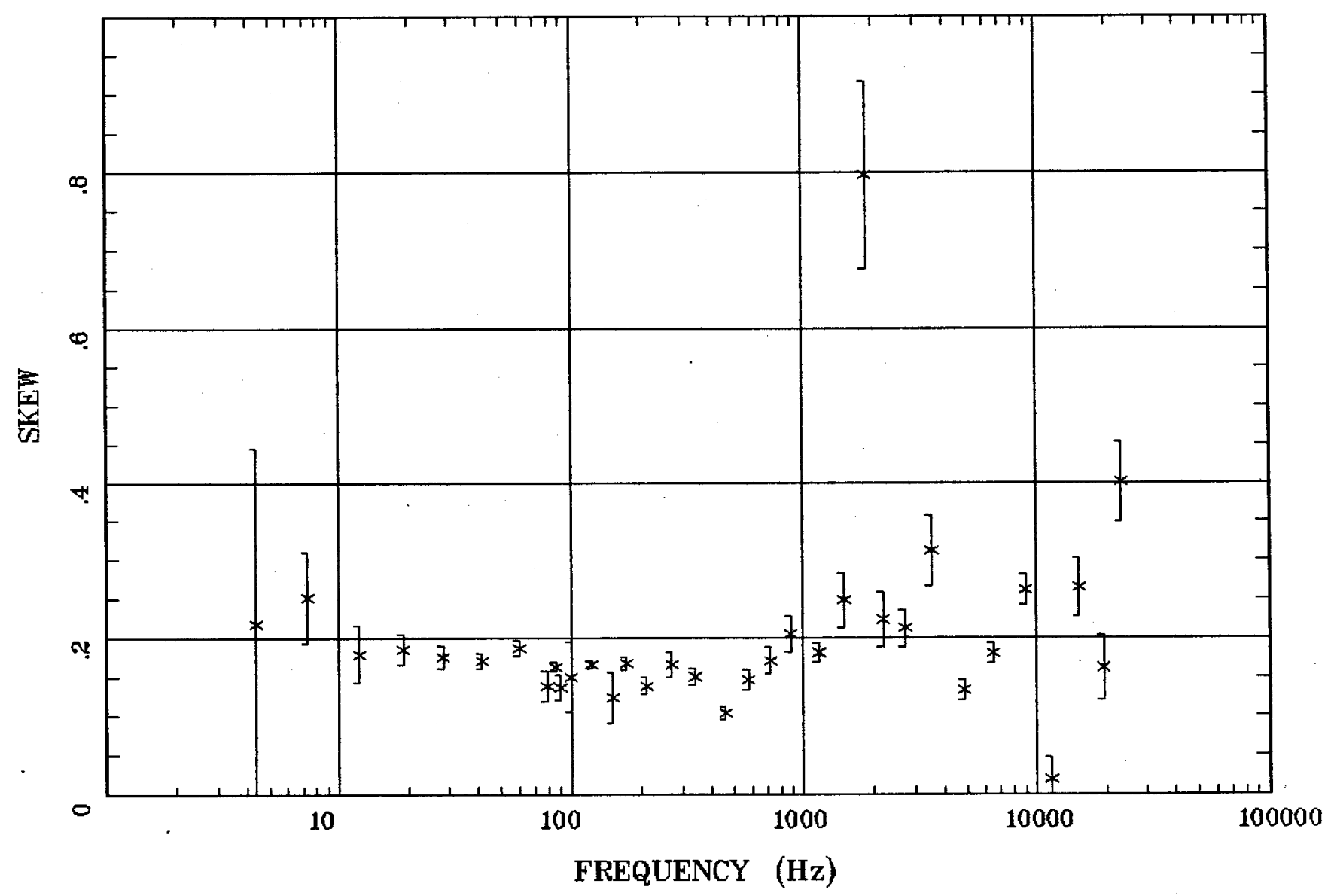

Client: Espanola Basin

Remote: none

Acquired: 16:0 Jul 13, 2004 Survey Co:USGS
Rotation:

Filename: cp33aall.avg

Channels: Ch1 Ch2 Ch3 Ch4 Ch5 Ch3 Ch4

Plotted: 15:01 Aug 18, 2004

< EMI - ElectroMagnetic Instruments 


\section{Station 33}

E MULT Coh.

Los Alamos, NM 100k

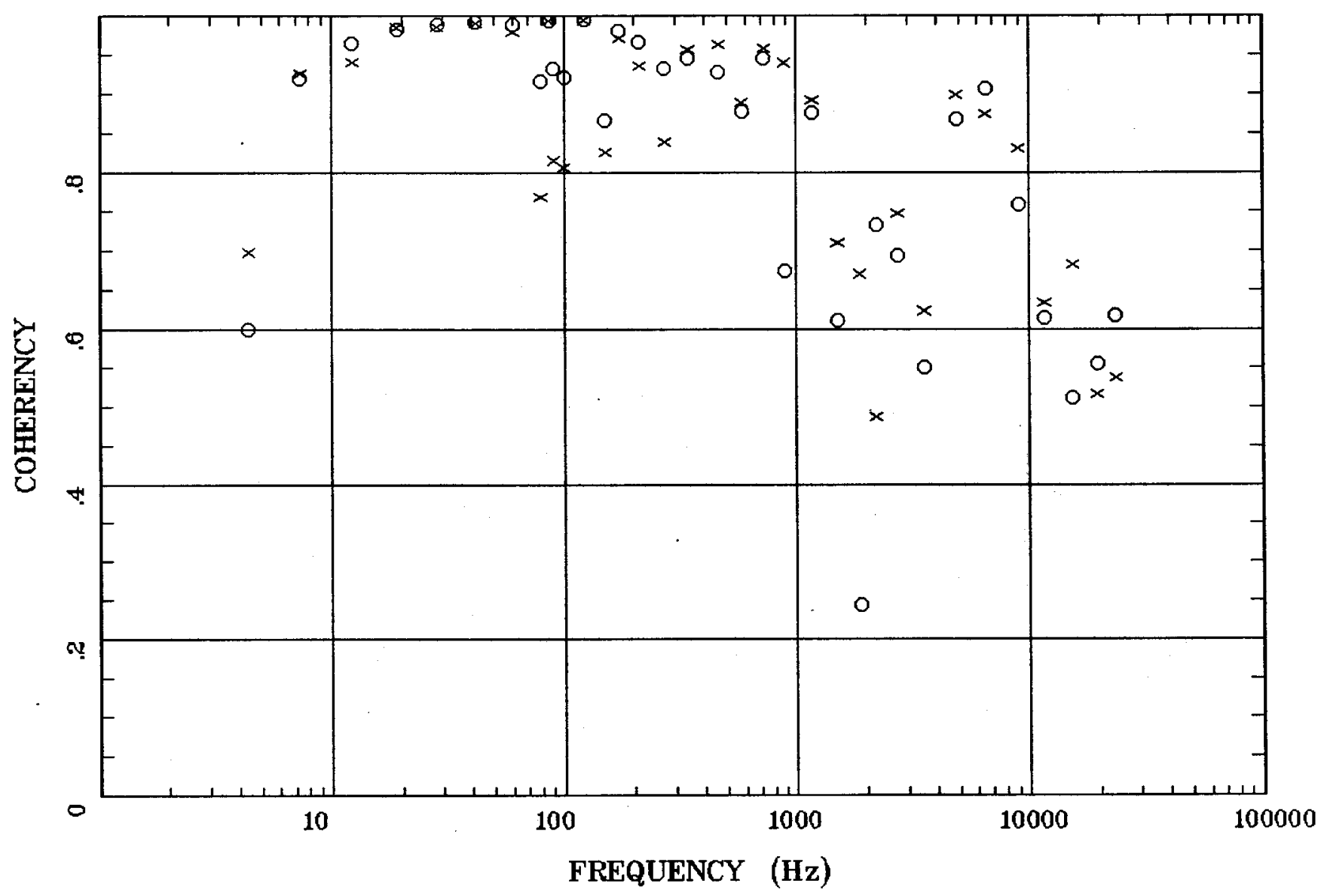

Client: Espanala Basin

Remote: none

Acquired: 16:0 Jul 13, 2004

Survey Co:USGS
Rotation:

Filename: cp33aall.avg

Channels: Ch1 Ch2 Ch3 Ch4 Ch5 Ch3 Ch4

Plotted: 15:01 Aug 18, 2004

< EMI - ElectroMagnetic Instruments 


\section{POLAR PLOTS}

Los Alamos, NM 100k

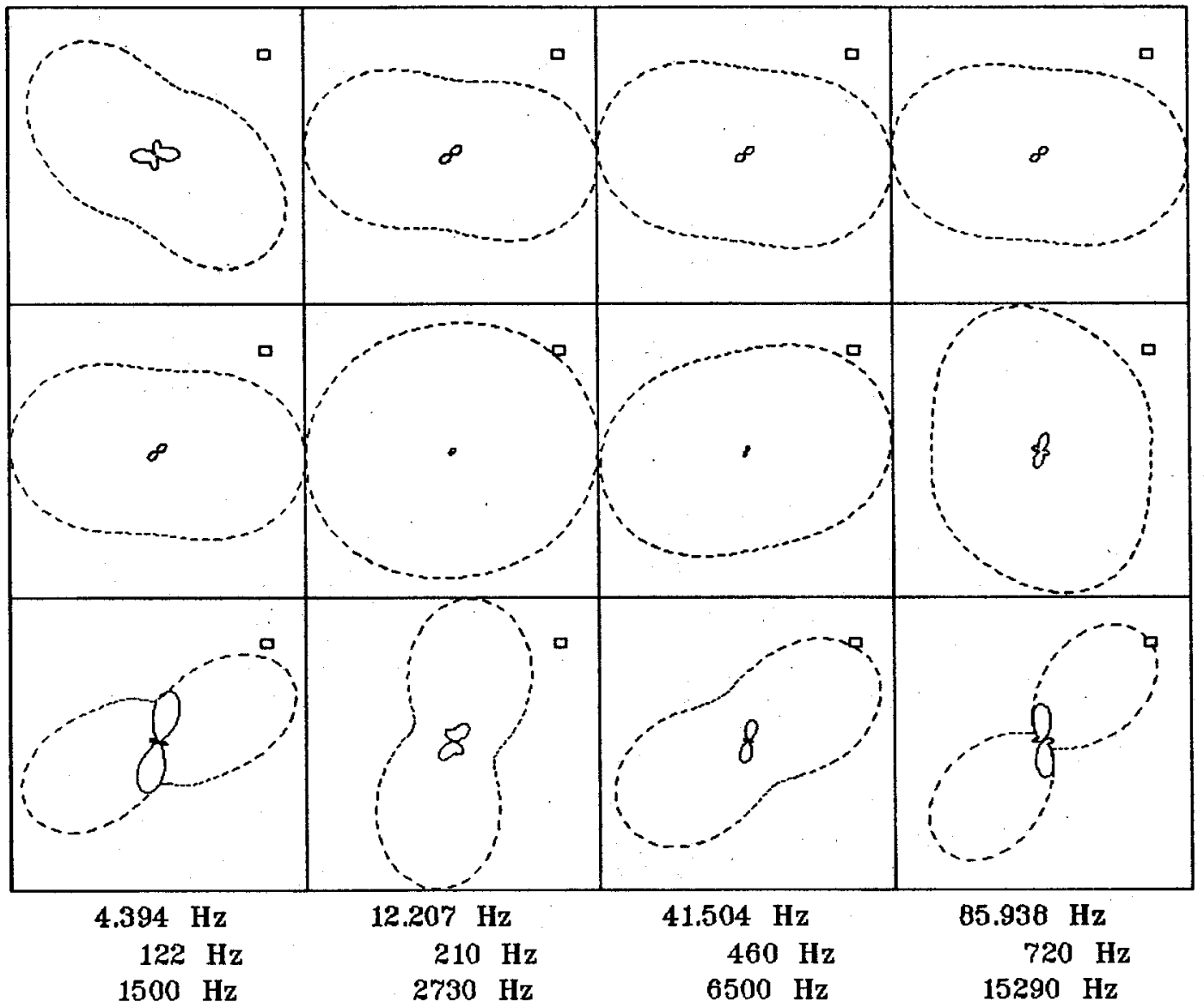

Client: Espanola Basin

Rotation:

Remote: none

Filename: cp33aall.avg

Channels: Ch1 Ch2 Ch3 Ch4 Ch5 Ch3 Ch4

Acquired: 16:0 Jul 13, 2004

Plotted: 15:01 Aug 18, 2004

Survey Co:USGS

< EMI - ElectroMagnetic Instruments 


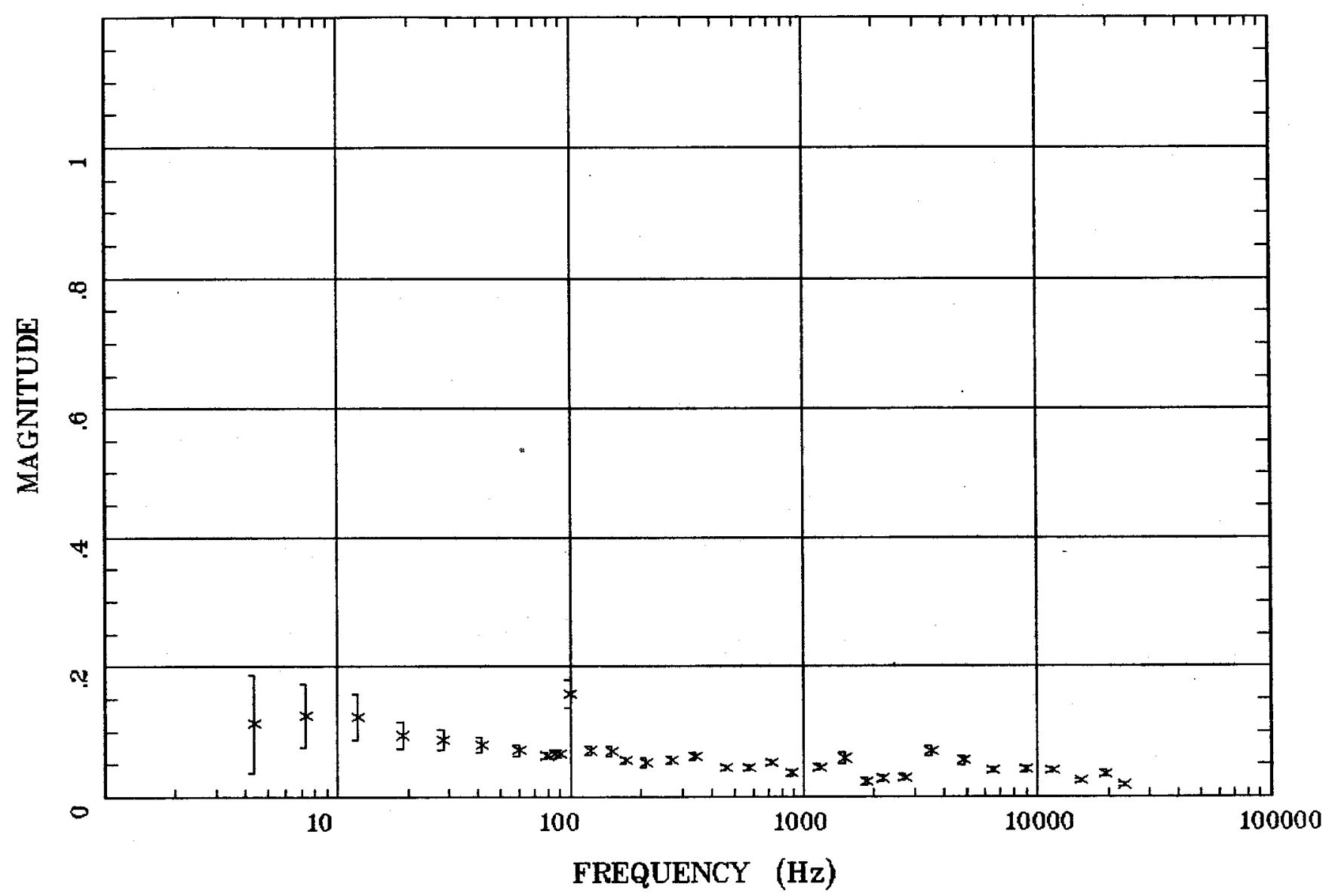

Client: Espanola Basin

Remote: none

Acquired: $16: 0$ Jul 13, 2004 Survey Co:USGS

\section{Rotation:}

Filename: cp33aall.avg

Channels: Ch1 Ch2 Ch3 Ch4 Ch5 Ch3 Ch4 Plotted: 15:01 Aug 18, 2004

< EMI - ElectroMagnetic Instruments 
Los Alamos, NM 100k

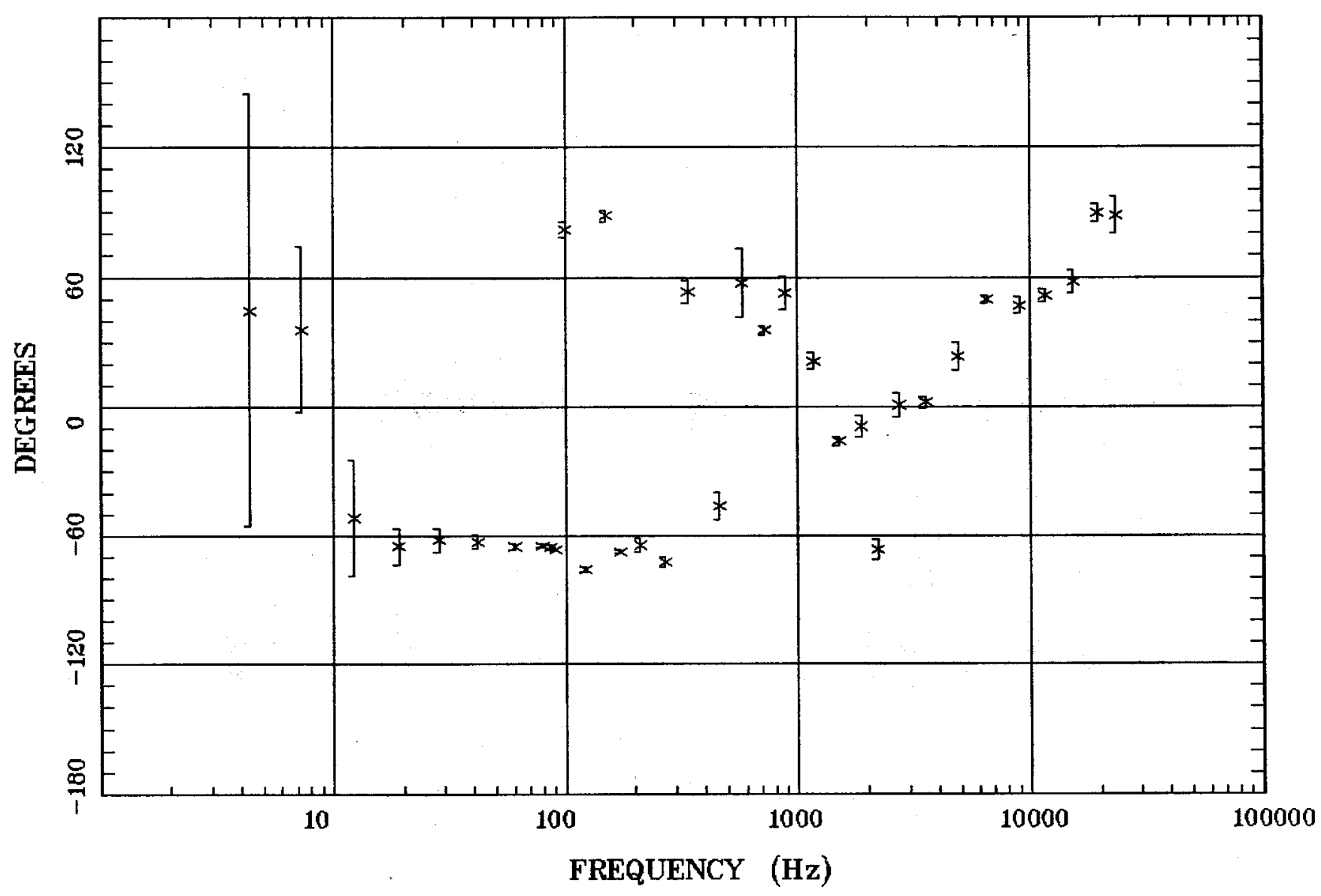

Client: Espanola Basin Remote: none Acquired: 16:0 Jul 13, 2004 Survey Co:USGS
Rotation:

Filename: cp33aall.avg

Channels: Ch1 Ch2 Ch3 Ch4 Ch5 Ch3 Ch4 Plotted: 15:01 Aug 18, 2004

< EMI - ElectroMagnetic Instrumients > 


\section{Station 33}

HzHx.x Coh HzHy.o

Los Alamos, NM 100k

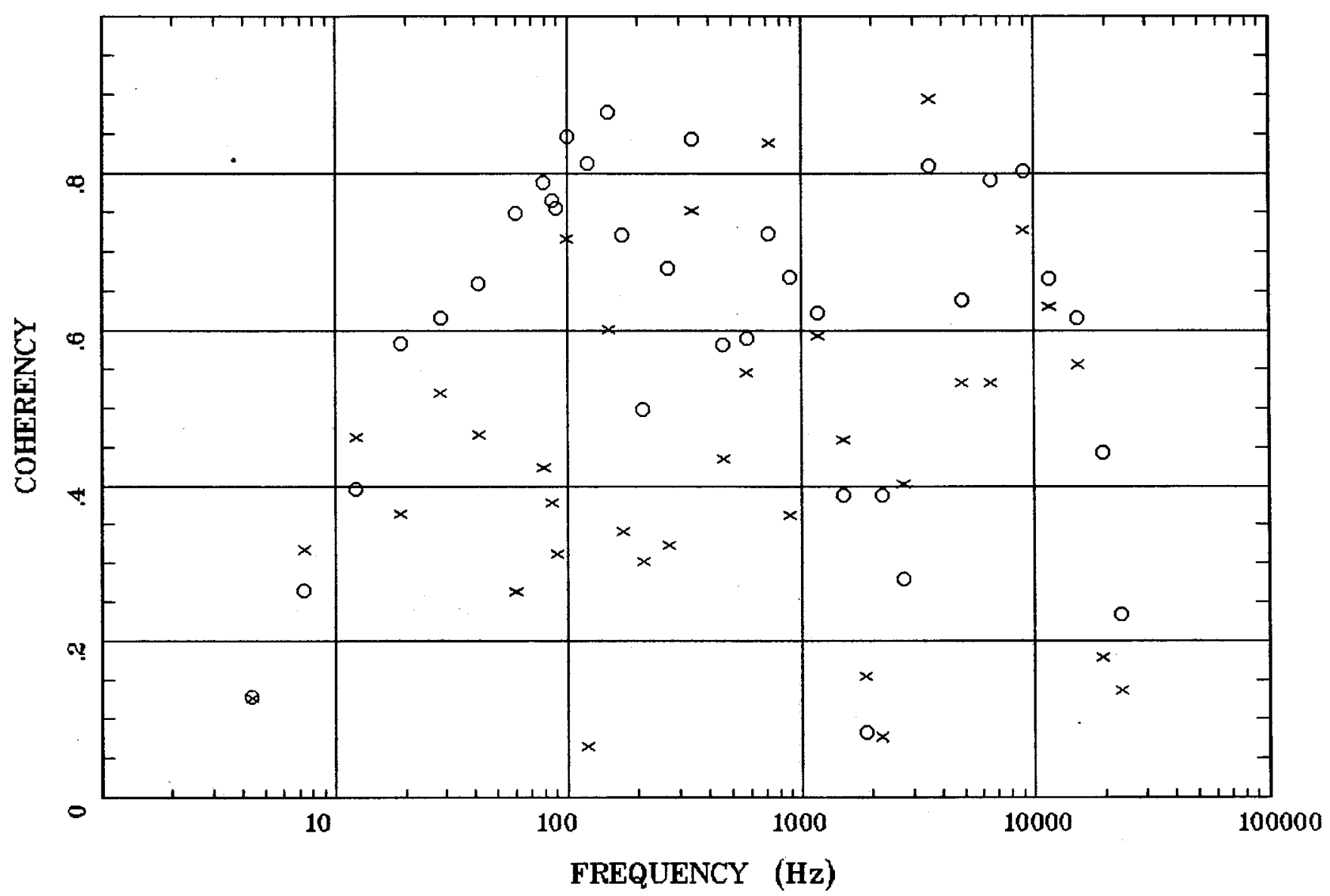

Client: Espanola Basin

Remote: none

Acquired: 16:0 Jul 13, 2004 Survey Co:USGS
Rotation:

Filename: cp33aallavg

Channels: Ch1 Ch2 Ch3 Ch4 Ch5 Ch3 Ch4

Plotted: 15:01 Aug 18, 2004

< EMI - ElectroMagnetic Instruments 


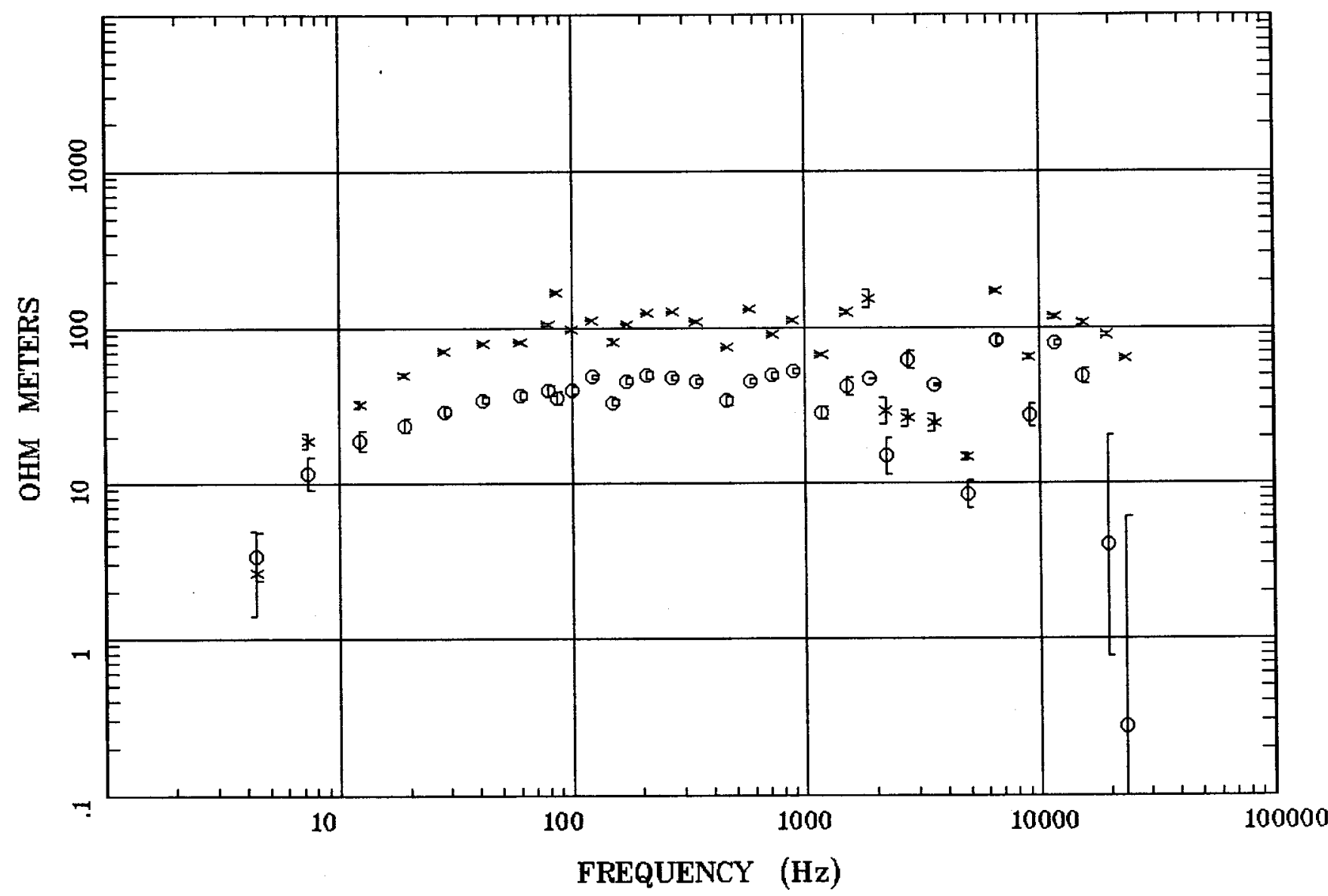

Client: Espanola Basin Remote: none Acquired: 13:1 Jul 15, 2004 Survey Co:USGS
Rotation:

Filename: cp32a.avg

Channels: Ch1 Ch2 Ch3 Ch4 Ch5 Ch3 Ch4

Plotted: 15:02 Aug 18, 2004

< EMI - ElectroMagnetic Instruments > 
IMPEDANCE PHASE

Los Alamos, NM 100k

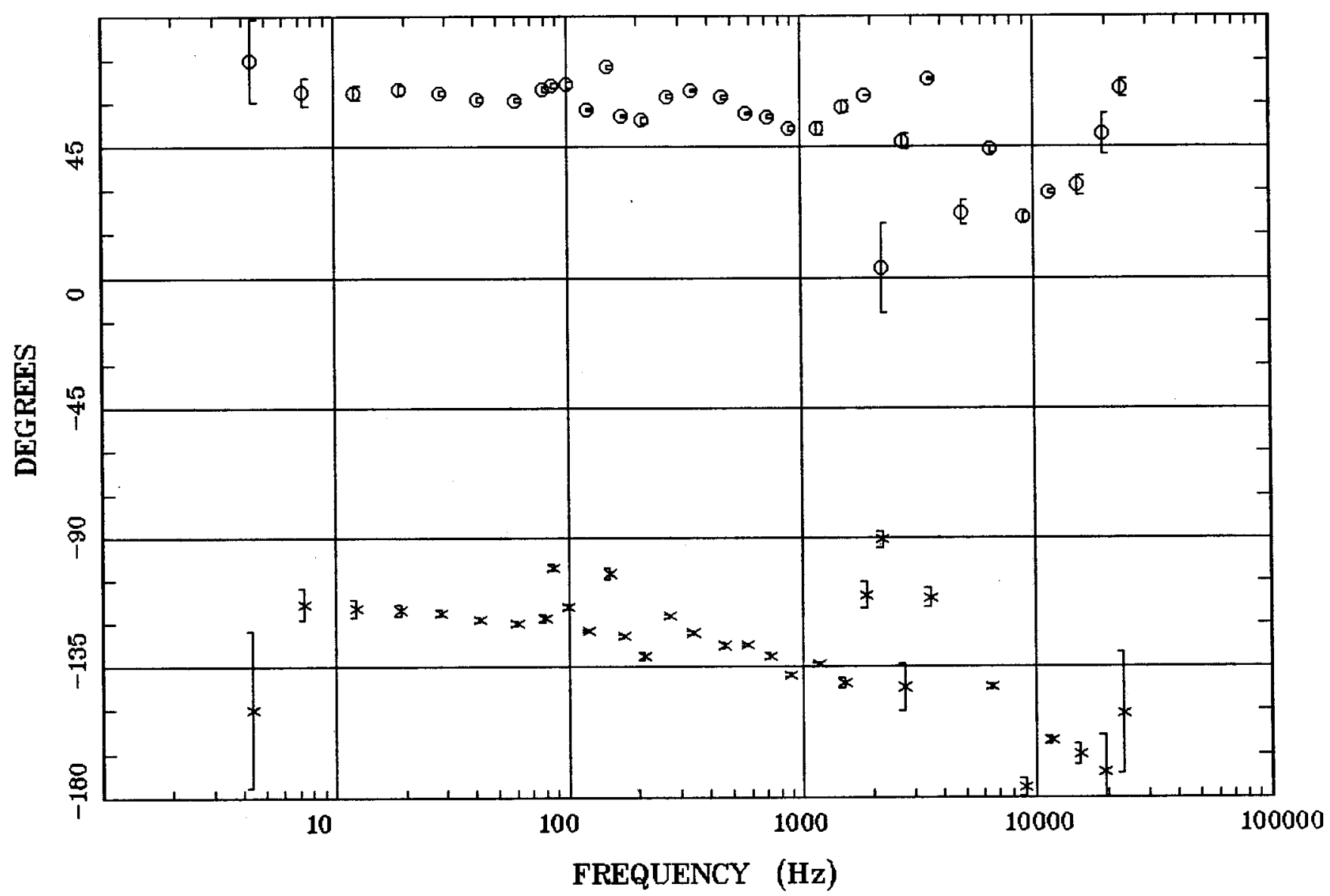

Client: Espanola Basin

Remote: none

Acquired: 13:1 Jul 15, 2004

Survey Co:USGS
Rotation:

Filename: cp32a.avg

Channels: Ch1 Ch2 Ch3 Ch4 Ch5 Ch3 Ch4

Plotted: 15:02 Aug 18, 2004

< EMI - ElectroMagnetic Instruments > 


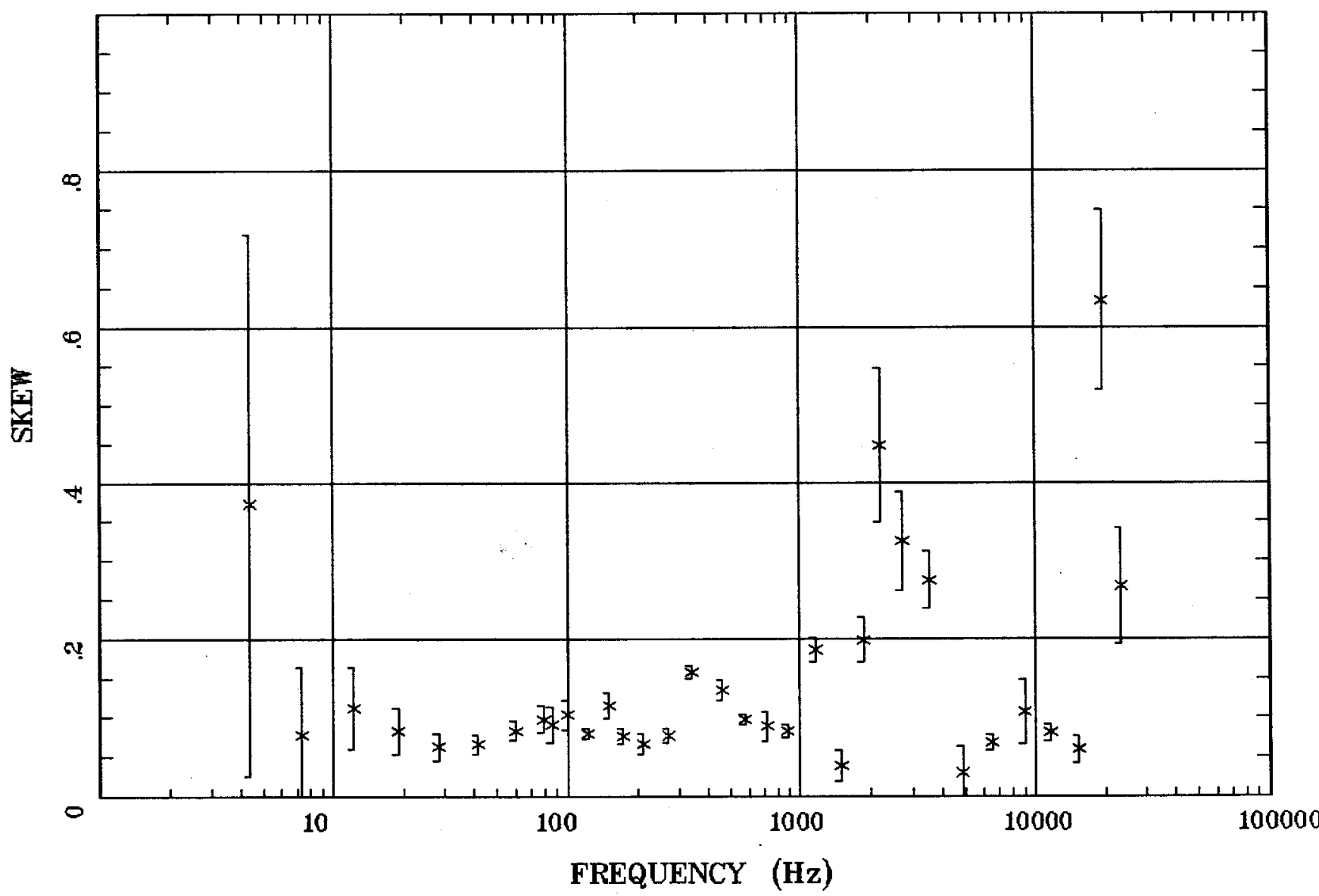

Client: Espanola Basin Remote: none Acquired: 13:1 Jul 15, 2004 Survey Co:USGS
Rotation:

Filename: cp32a.avg Channels: Ch1 Ch2 Ch3 Ch4 Ch5 Ch3 Ch4 Plotted: 15:02 Aug 18, 2004

< EMI - ElectroMagnetic Instruments > 
E MULT Coh.

Los Alamos, NM 100k

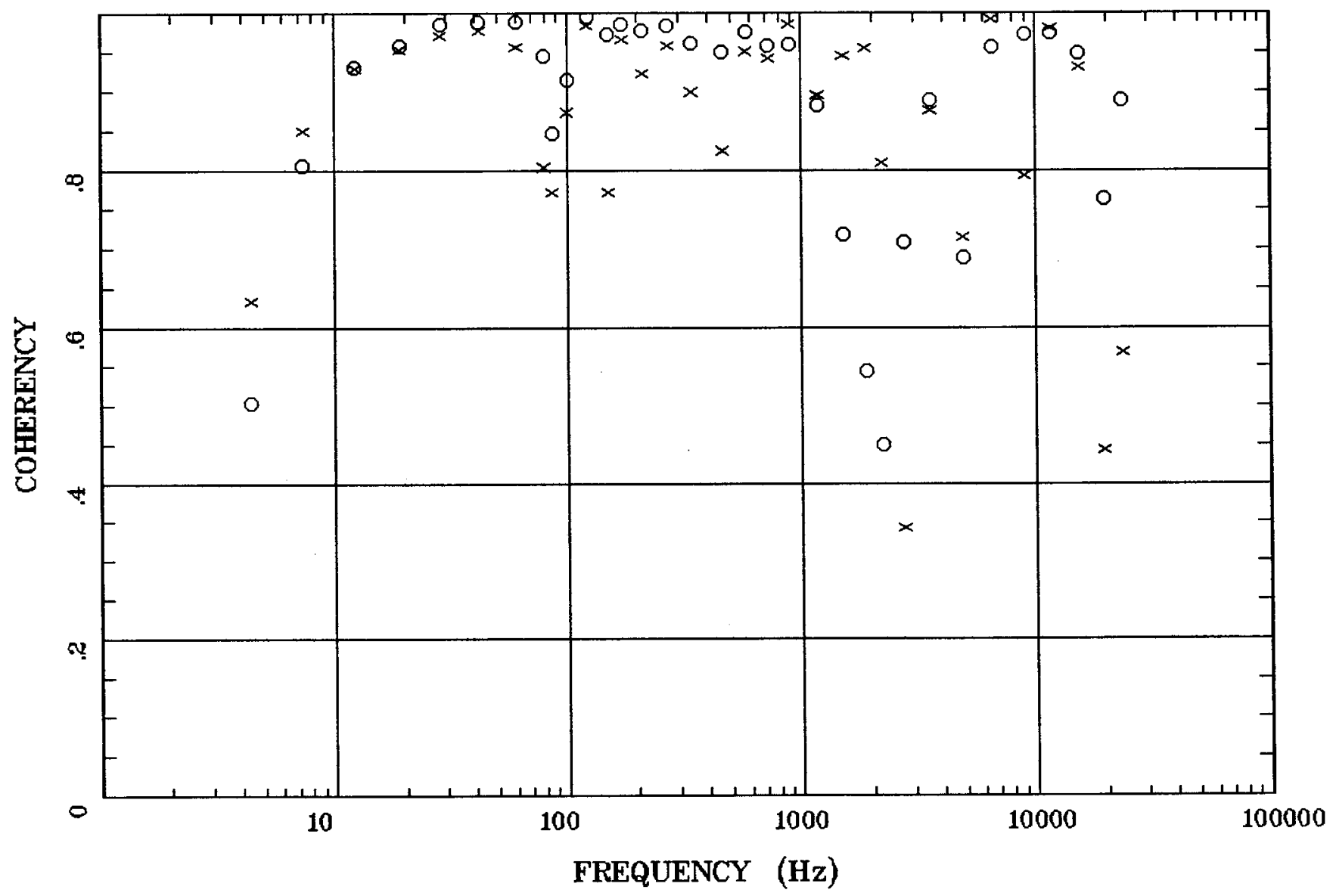

Client: Espanola Basin Remote: none

Acquired: 13:1 Jul 15, 2004

Survey Co:USGS
Rotation:

Filename: cp32a.avg

Channels: Ch1 Ch2 Ch3 Ch4 Ch5 Ch3 Ch4

Plotted: 15:02 Aug 18, 2004

< EMI - ElectroMagnetic Instruments > 
POLAR PLOTS

\section{Los Alamos, NM 100k}

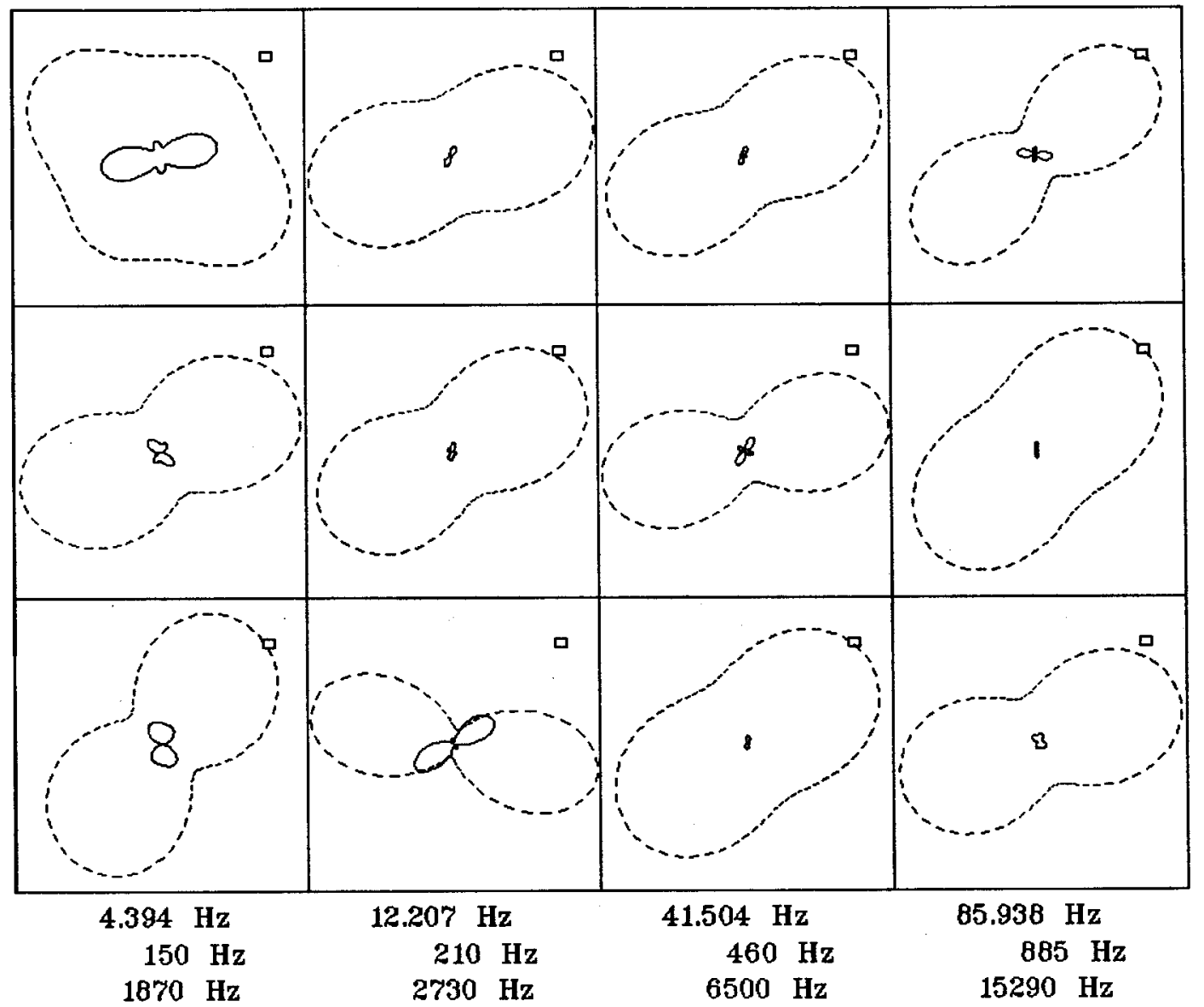

Client: Espanola Basin

Rotation:

Remote: none

Filename: cp32a.avg

Channels: Ch1 Ch2 Ch3 Ch4 Ch5 Ch3 Ch4 Acquired: 13:1 Jul 15, 2004 Survey Co:USGS

Plotted: 15:02 Aug 18, 2004

< EMI - ElectroMagnetic Instruments > 


\section{Station 32}

TIPPER MAGNITUDE

Los Alamos, NM 100k

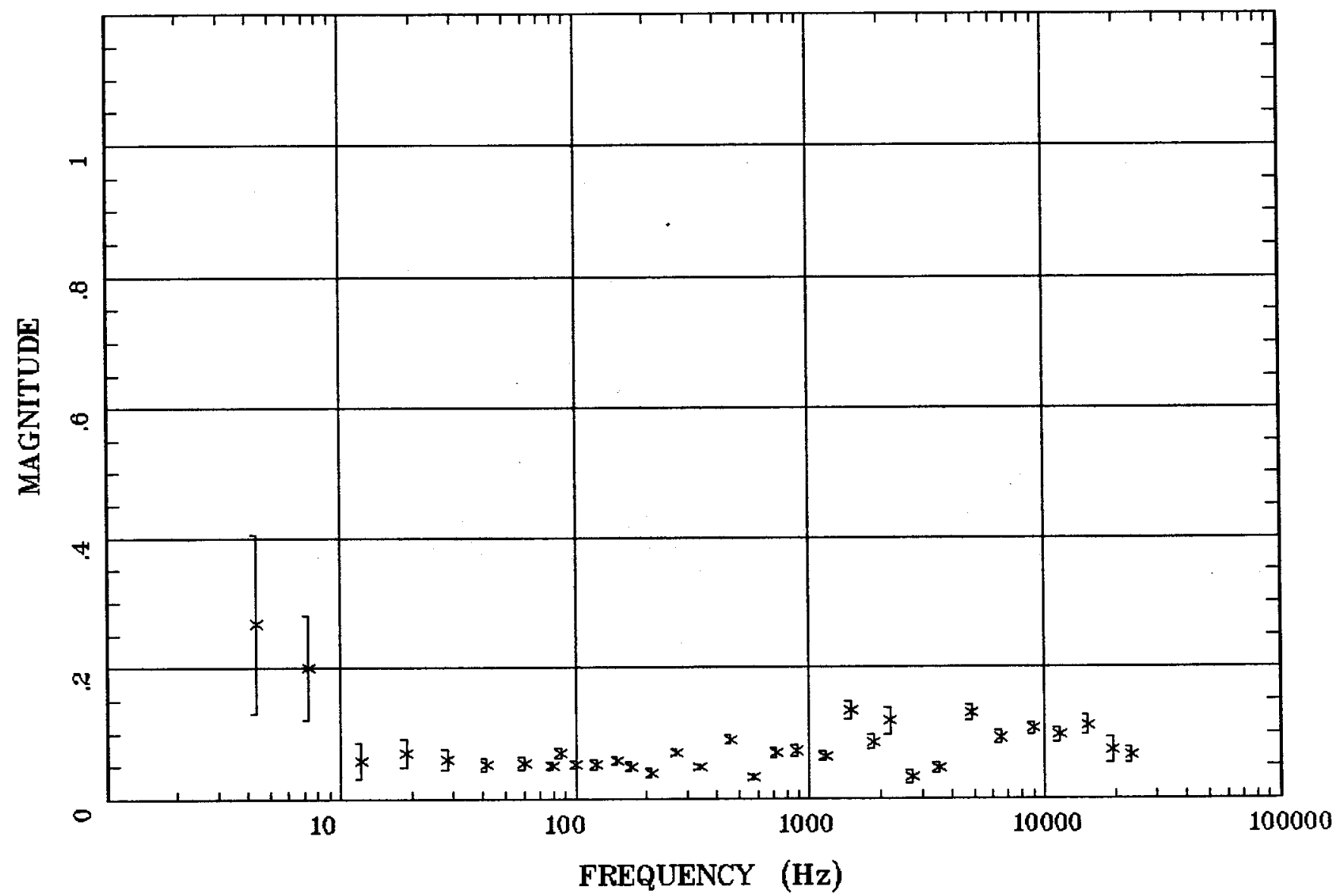

Client: Espanola Basin

Remote: none

Acquired: 13:1 Jul 15, 2004

Survey Co:USGS
Rotation:

Filename: cp32a.avg

Channels: Ch1 Ch2 Ch3 Ch4 Ch5 Ch3 Ch4

Plotted: 15:02 Aug 18, 2004

$<$ EMI - ElectroMagnetic Instruments > 
Los Alamos, NM 100k

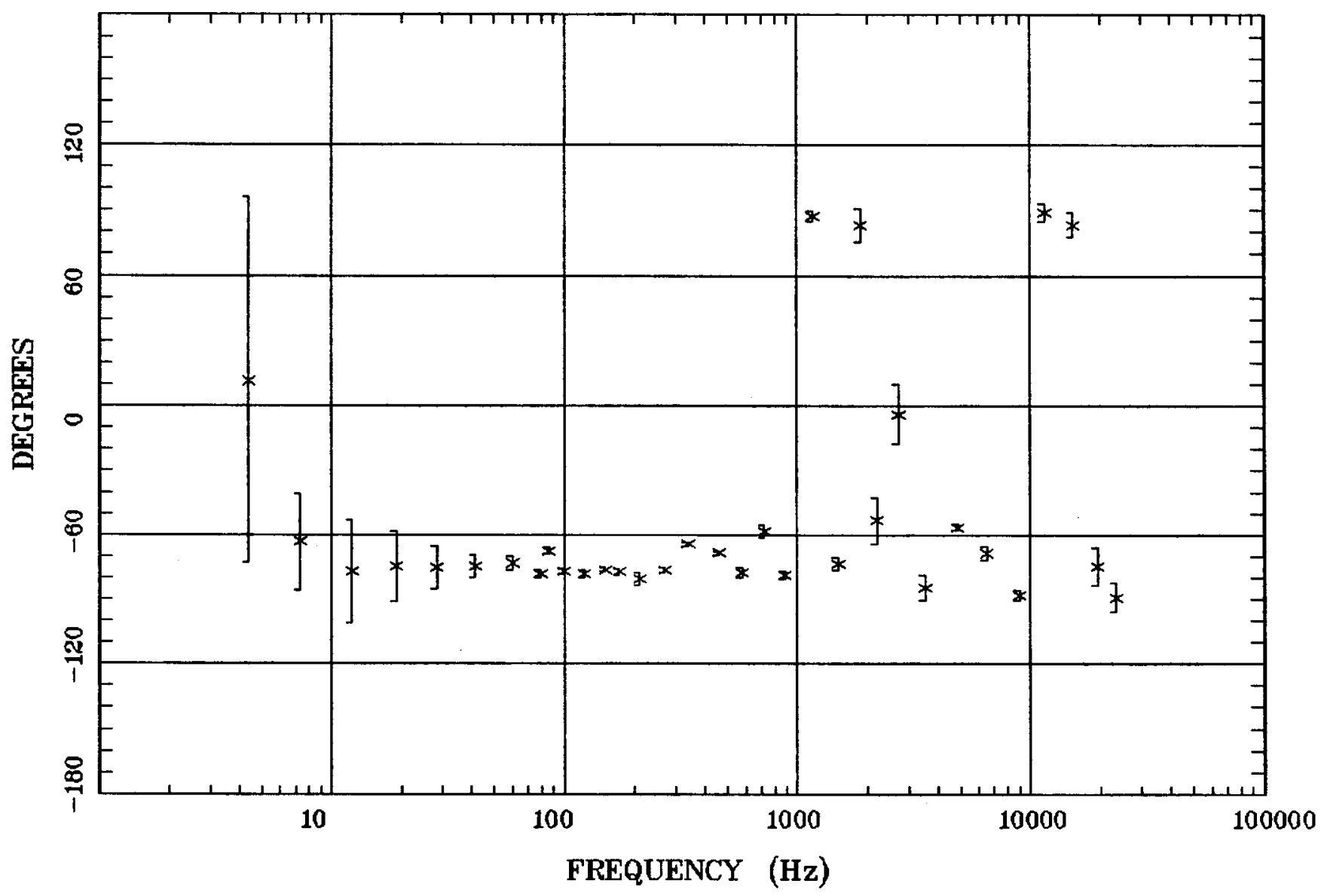

Client: Espanola Basin

Remote: none

Acquired: 13:1 Jul 15, 2004 Survey Co:USGS
Rotation:

Filename: cp32a.avg

Channels: Ch1 Ch2 Ch3 Ch4 Ch5 Ch3 Ch4 Plotted: 15:02 Aug 18, 2004

< EMI - ElectroMagnetic Instruments 
HzHx.x Coh HzHy.o

Los Alamos, NM 100k

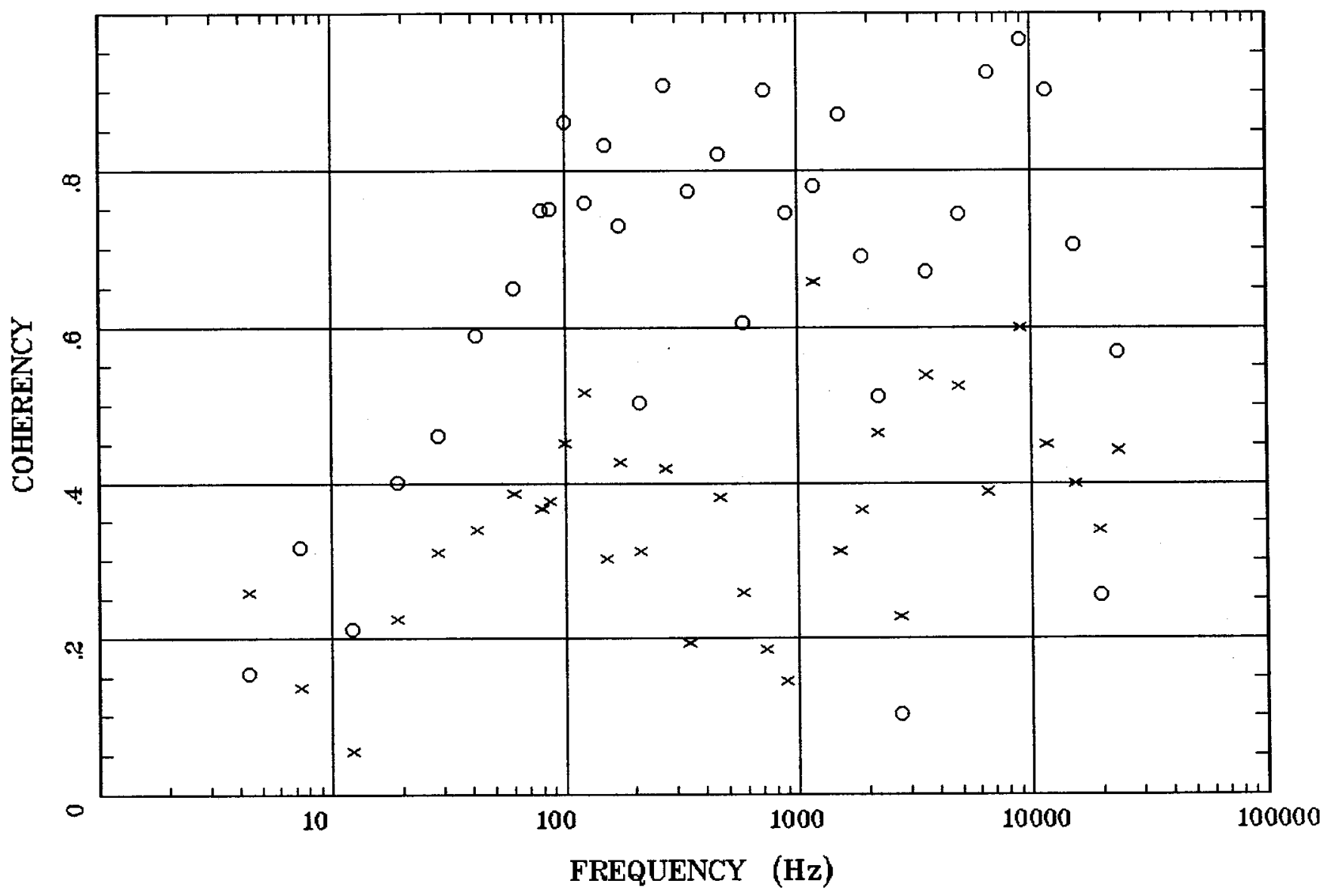

Client: Espanola Basin

Remote: none

Acquired: 13:1 Jul 15, 2004 Survey Co:USGS
Rotation:

Filename: cp32a.avg

Channels: Ch1 Ch2 Ch3 Ch4 Ch5 Ch3 Ch4

Plotted: 15:02 Aug 18, 2004

< EMI - ElectroMagnetic Instruments 


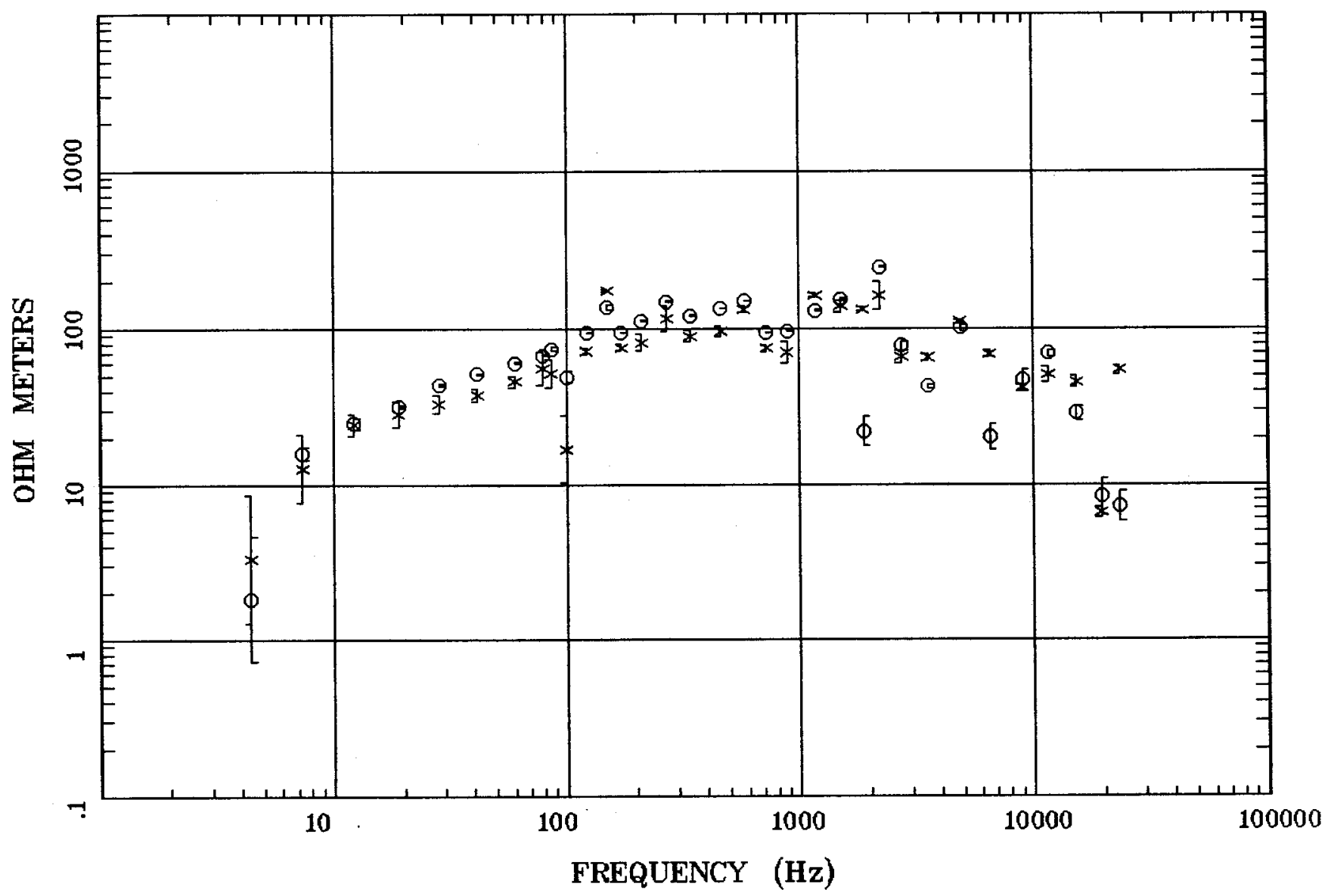

Client: Espanola Basin Remote: none

Acquired: 13:5 Jul 14, 2004 Survey Co:USGS
Rotation:

Filename: cp31a.avg

Channels: Ch1 Ch2 Ch3 Ch4 Ch5 Ch3 Ch4

Plotted: 10:29 Aug 23, 2004

< EMI - ElectroMagnetic Instruments > 
IMPEDANCE PHASE

Los Alamos, NM 100k

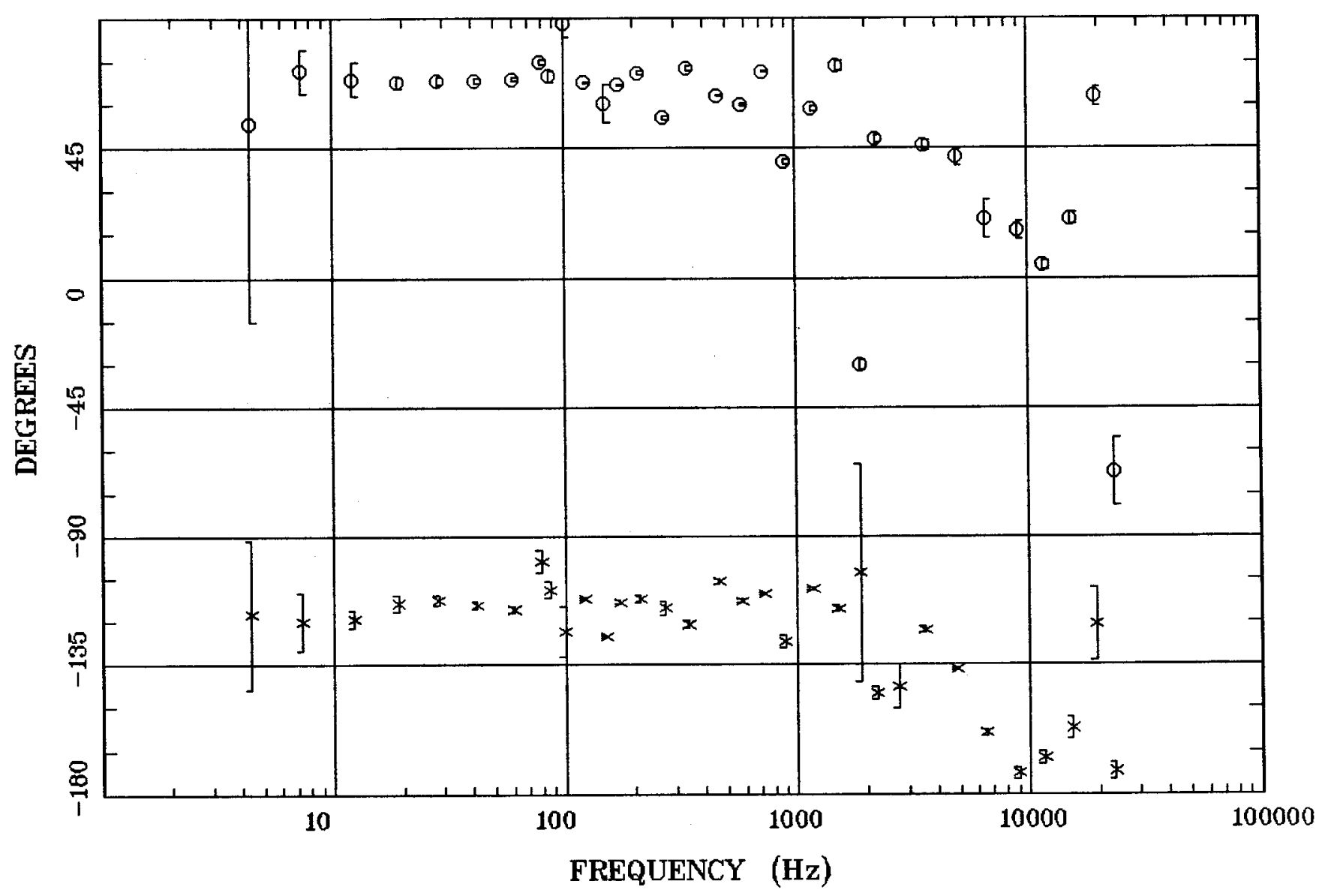

Client: Espanola Basin

Remote: none

Acquired: $13: 5$ Jul 14, 2004 Survey Co:USGS
Rotation:

Filename: cp31a.avg

Channels: Ch1 Ch2 Ch3 Ch4 Ch5 Ch3 Ch4

Plotted: 10:29 Aug 23, 2004

< EMI - ElectroMagnetic Instruments > 


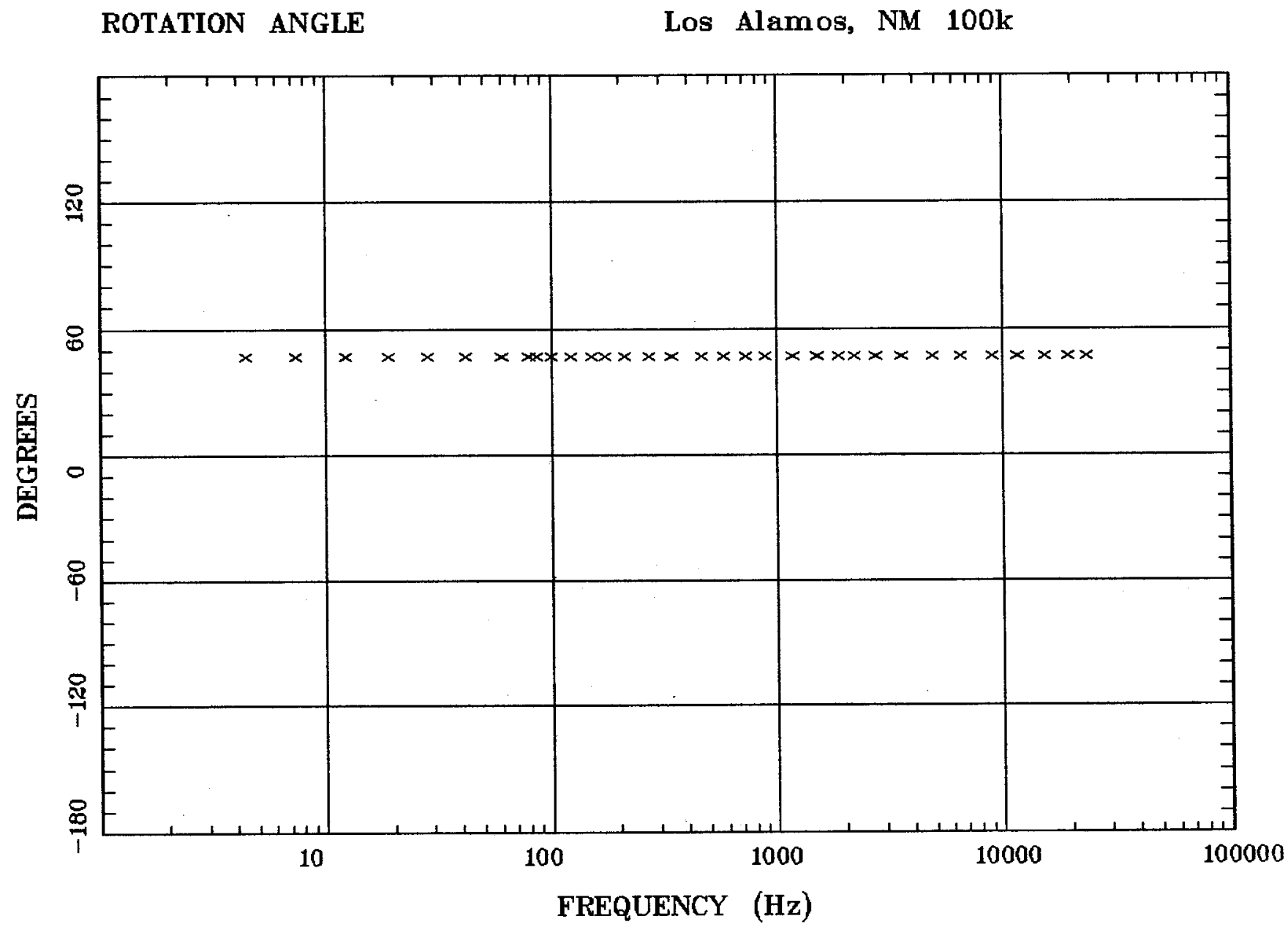

Client: Espanola Basin Remote: none

Acquired: 13:5 Jul 14, 2004 Survey Co:USGS
Rotation:

Filename: cp31a.avg

Channels: Ch1 Ch2 Ch3 Ch4 Ch5 Ch3 Ch4

Plotted: 10:29 Aug 23, 2004

< EMI - ElectroMagnetic Instruments > 


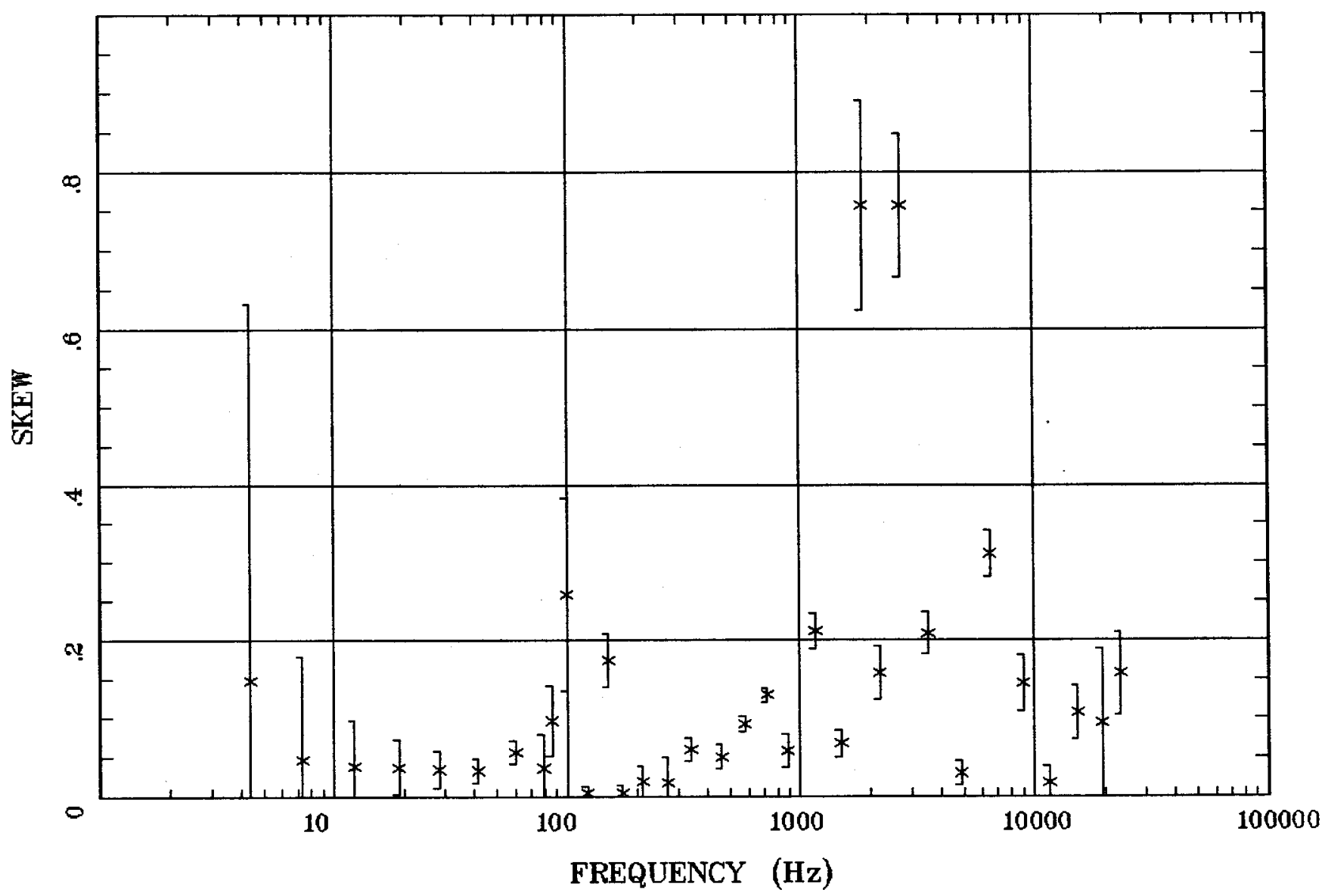

Client: Espanola Basin

Remote: none

Acquired: 13:5 Jul 14, 2004

Survey Co:USGS
Rotation:

Filename: cp31a.avg

Channels: Ch1 Ch2 Ch3 Ch4 Ch5 Ch3 Ch4 Plotted: 10:29 Aug 23, 2004

< EMI - ElectroMagnetic Instruments > 
E MULT Coh. Los Alamos, NM 100k

Station 31

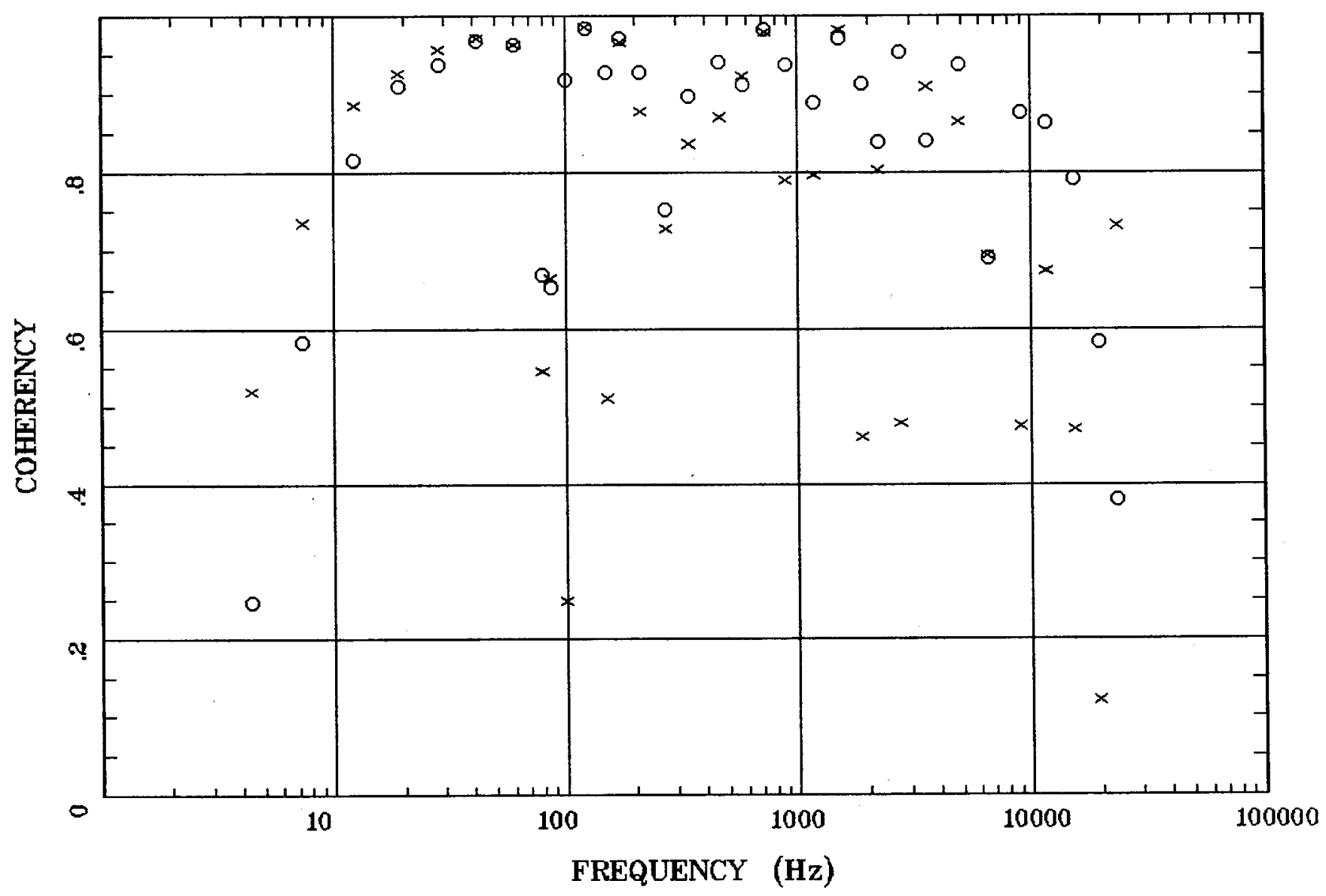

Client: Espanola Basin

Remote: none

Acquired: 13:5 Jul 14, 2004

Survey Co:USGS
Rotation:

Filename: cp31a.avg

Channels: Ch1 Ch2 Ch3 ch4 Ch5 Ch3 Ch4

Plotted: 10:29 Aug 23, 2004

< EMI - ElectroMagnetic Instruments > 


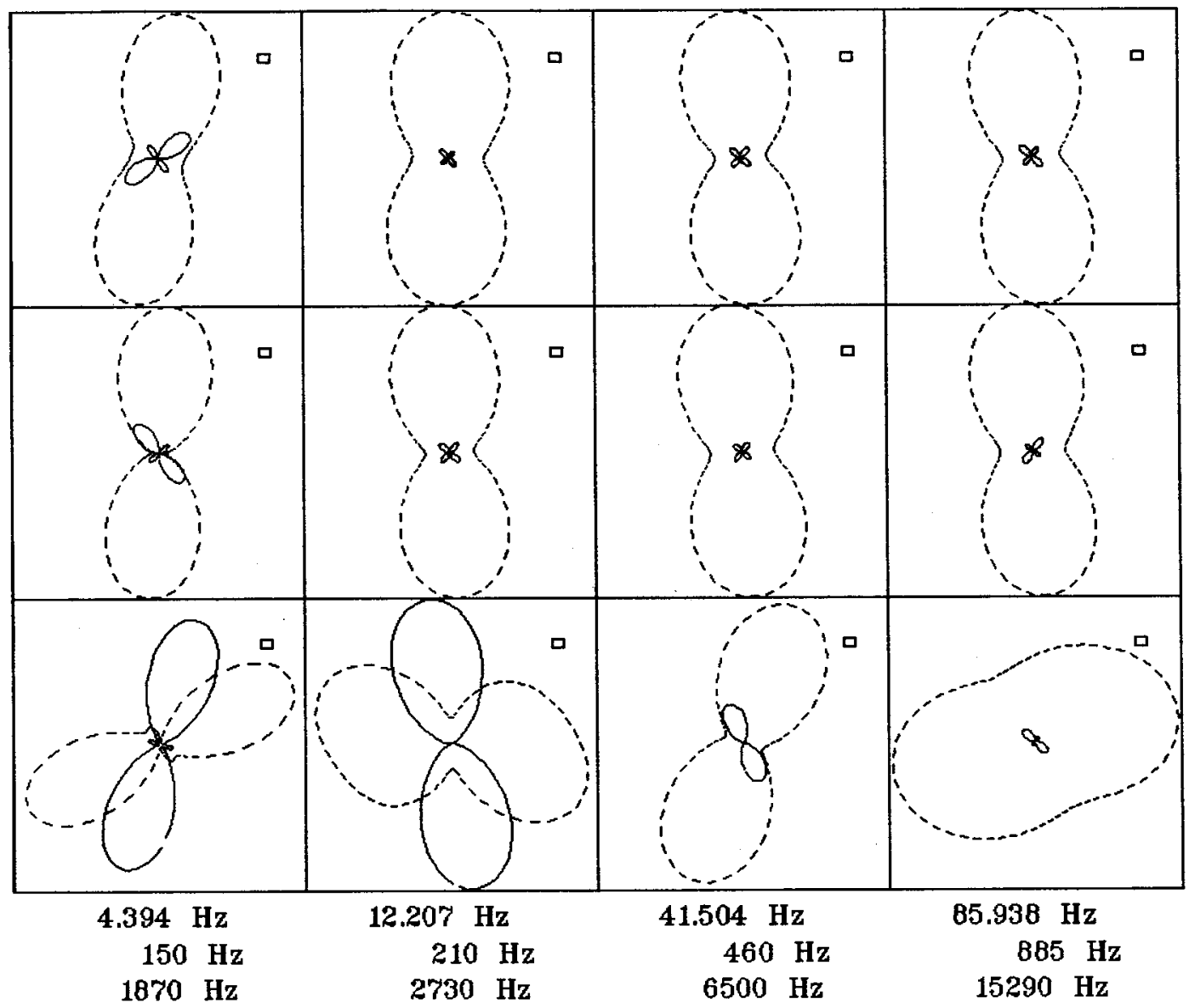

Client: Espanala Basin

Remote: none

Acquired: 13:5 Jul 14, 2004

Survey Co:USGS
Rotation:

Filename: cp31a.avg

Channels: Ch1 Ch2 Ch3 Ch4 Ch5 Ch3 Ch4

Plotted: 10:29 Aug 23, 2004

< EMI - ElectroMagnetic Instruments > 
Los Alamos, NM 100k

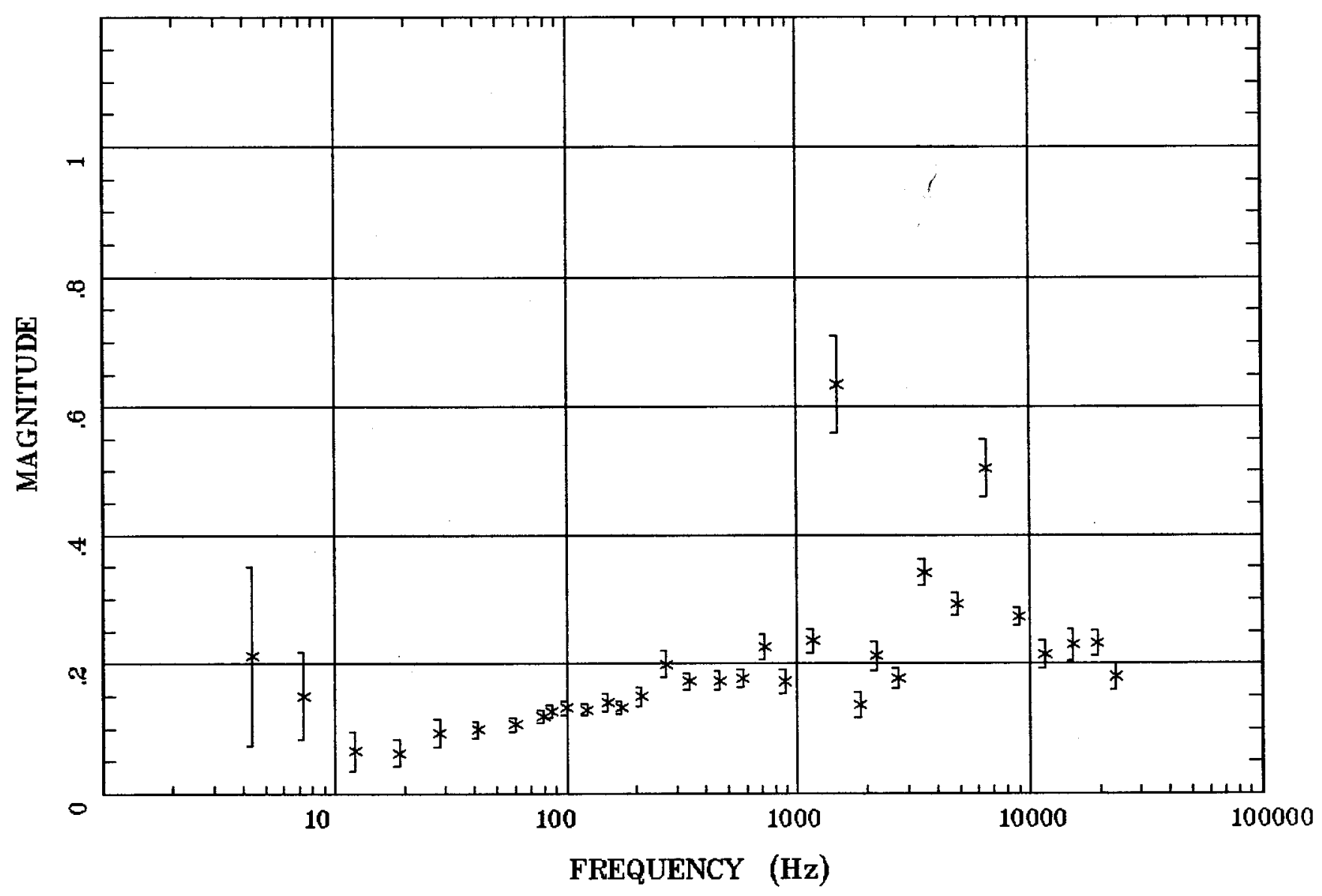

Client: Espanola Basin

Remote: none

Acquired: 13:5 Jul 14, 2004

Survey Co:USGS
Rotation:

Filename: cp31a.avg

Channels: Ch1 Ch2 Ch3 Ch4 Ch5 Ch3 Ch4 Plotted: 10:29 Aug 23, 2004

< EMI - ElectroMagnetic Instruments 


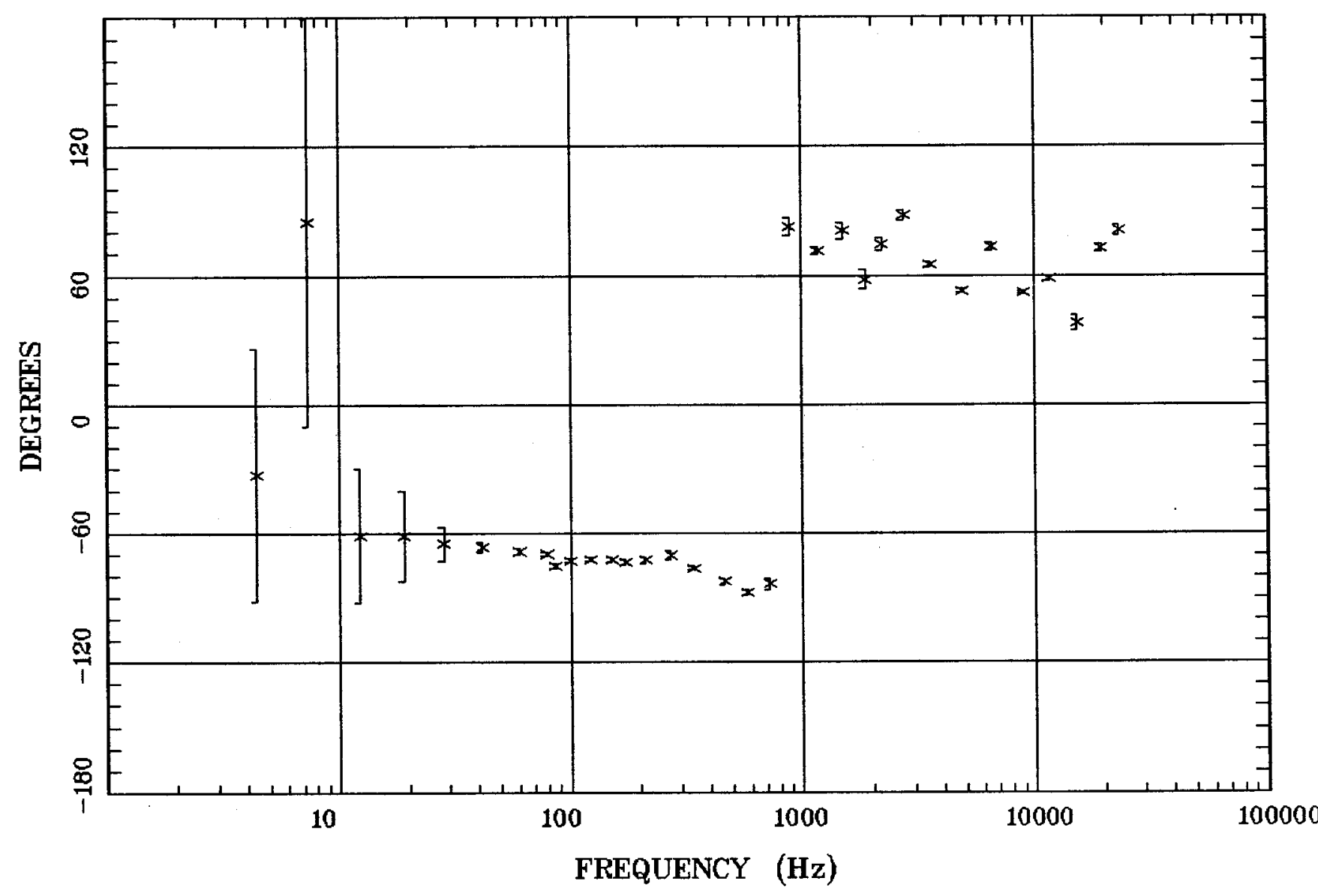

Client: Espanola Basin

Remote: none

Acquired: 13:5 Jul 14, 2004 Survey Co:USGS
Rotation:

Filename: cp31a.avg

Channels: Ch1 Ch2 Ch3 Ch4 Ch5 Ch3 Ch4 Plotted: 10:29 Aug 23, 2004

$<$ EMI - ElectroMagnetic Instruments 
Los Alamos, NM 100k

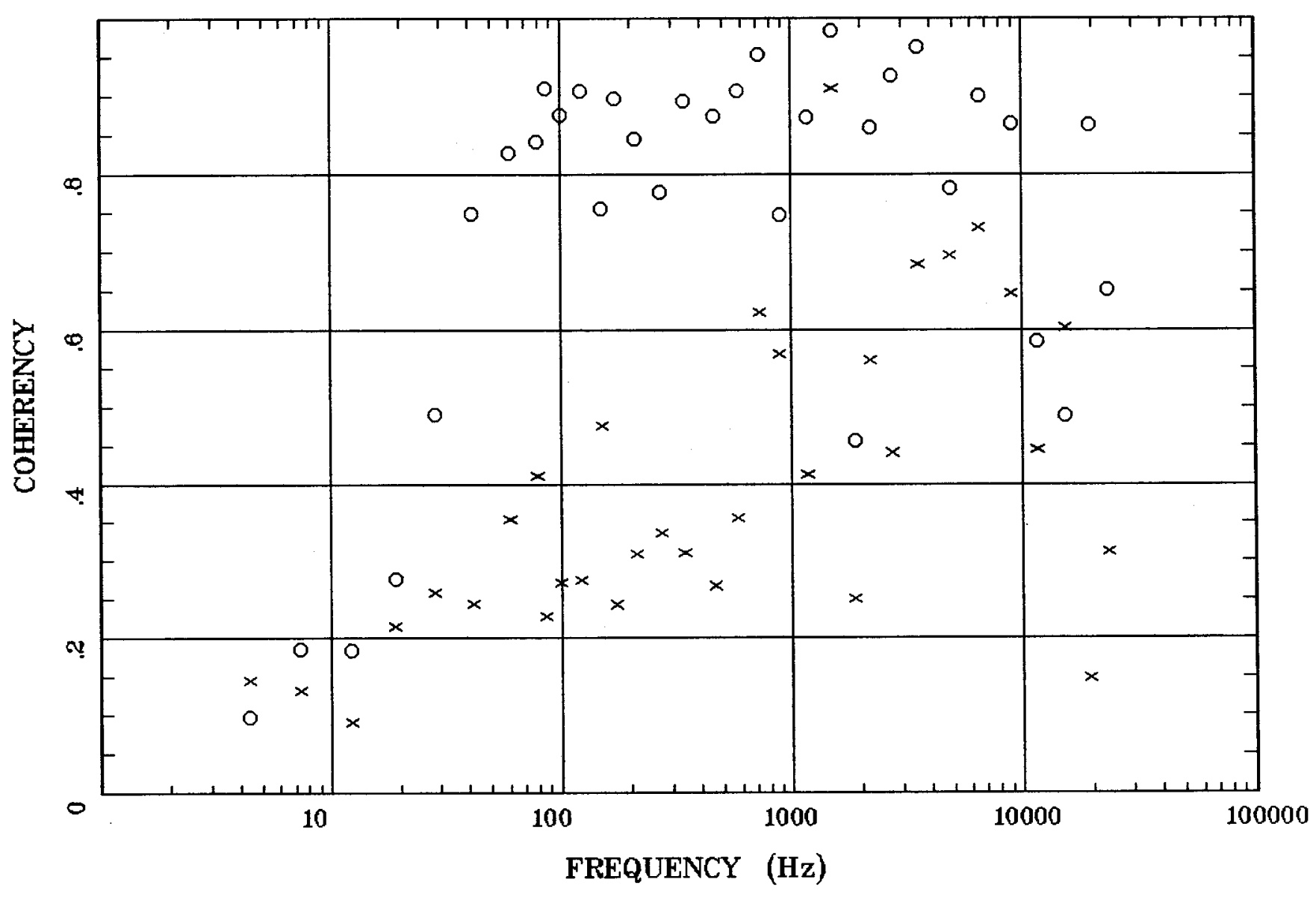

Client: Espanola Basin

Remote: none

Acquired: 13:5 Jul 14, 2004

Survey Co:USGS
Rotation:

Filename: cp31a.avg

Channels: Ch1 Ch2 Ch3 Ch4 Ch5 Ch3 Ch4

Plotted: 10:29 Aug 23, 2004

$<$ EMI - ElectroMagnetic Instruments 
Station $\mathbf{3 0}$

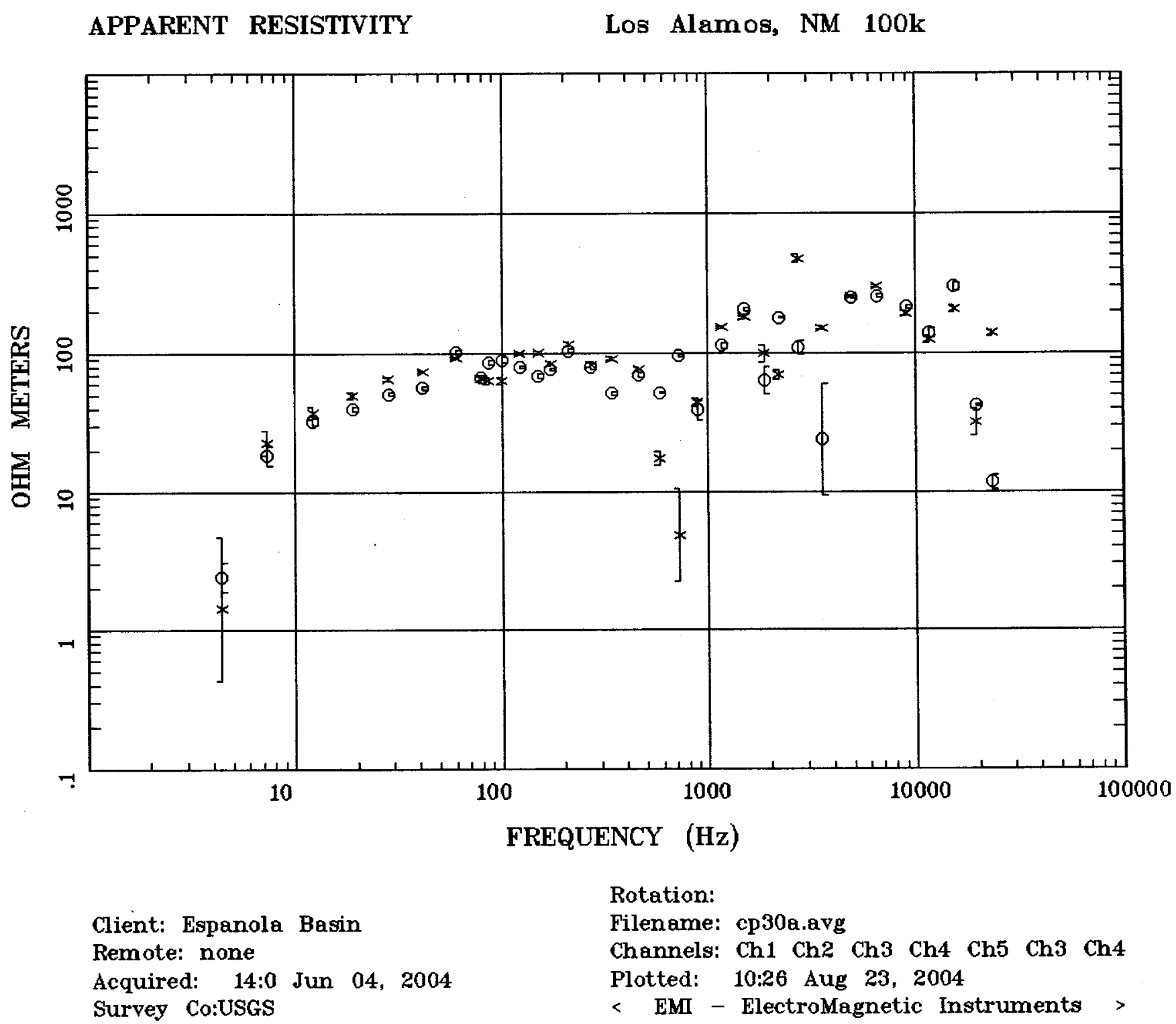


IMPEDANCE PHASE

Los Alamos, NM 100k

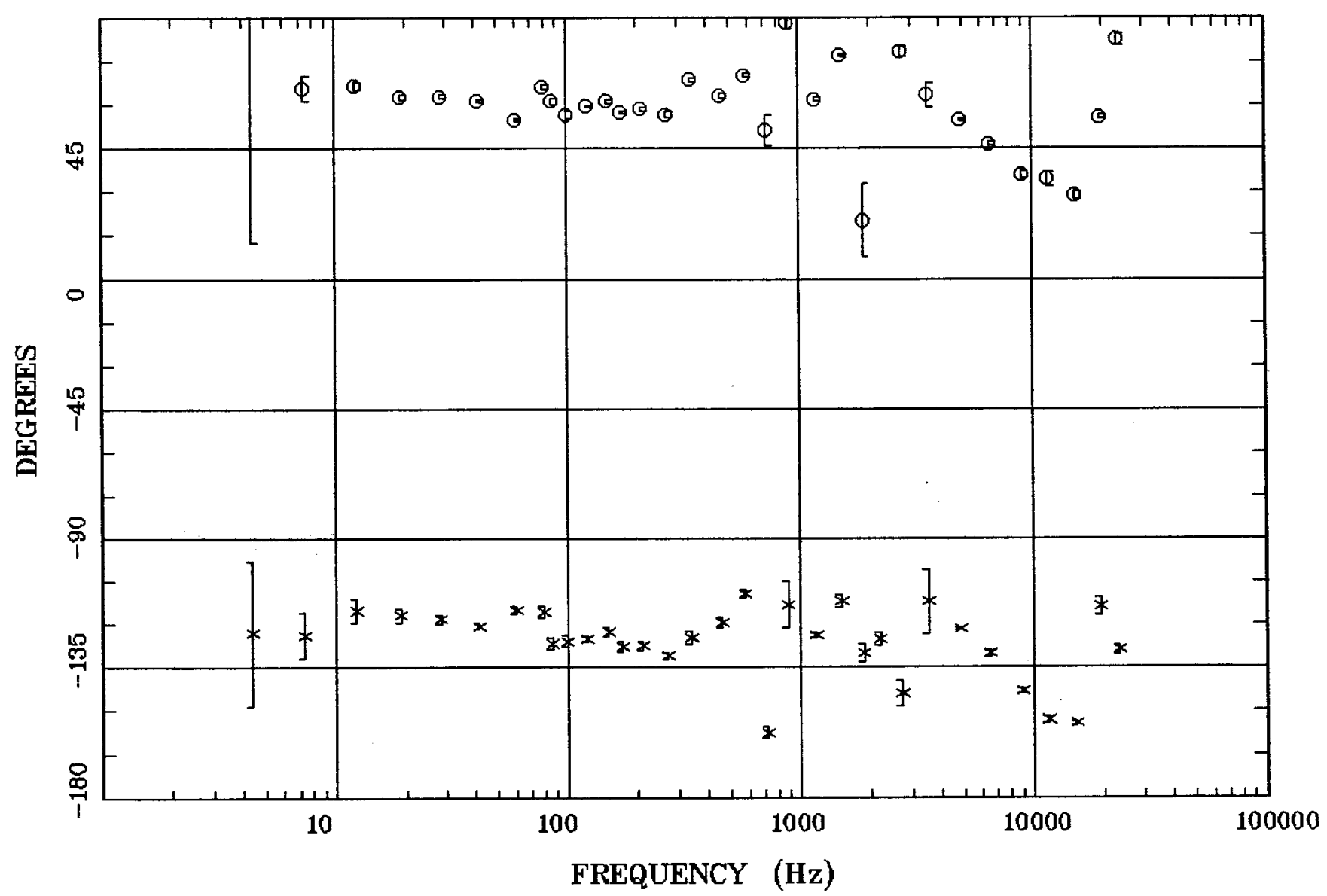

Client: Espanola Basin Remote: none Acquired: 14:0 Jun 04, 2004 Survey Co:USGS
Rotation:

Filename: cp30a.avg

Channels: Ch1 Ch2 Ch3 Ch4 Ch5 Ch3 Ch4 Plotted: 10:26 Aug 23, 2004

$<$ EMI - ElectroMagnetic Instruments 


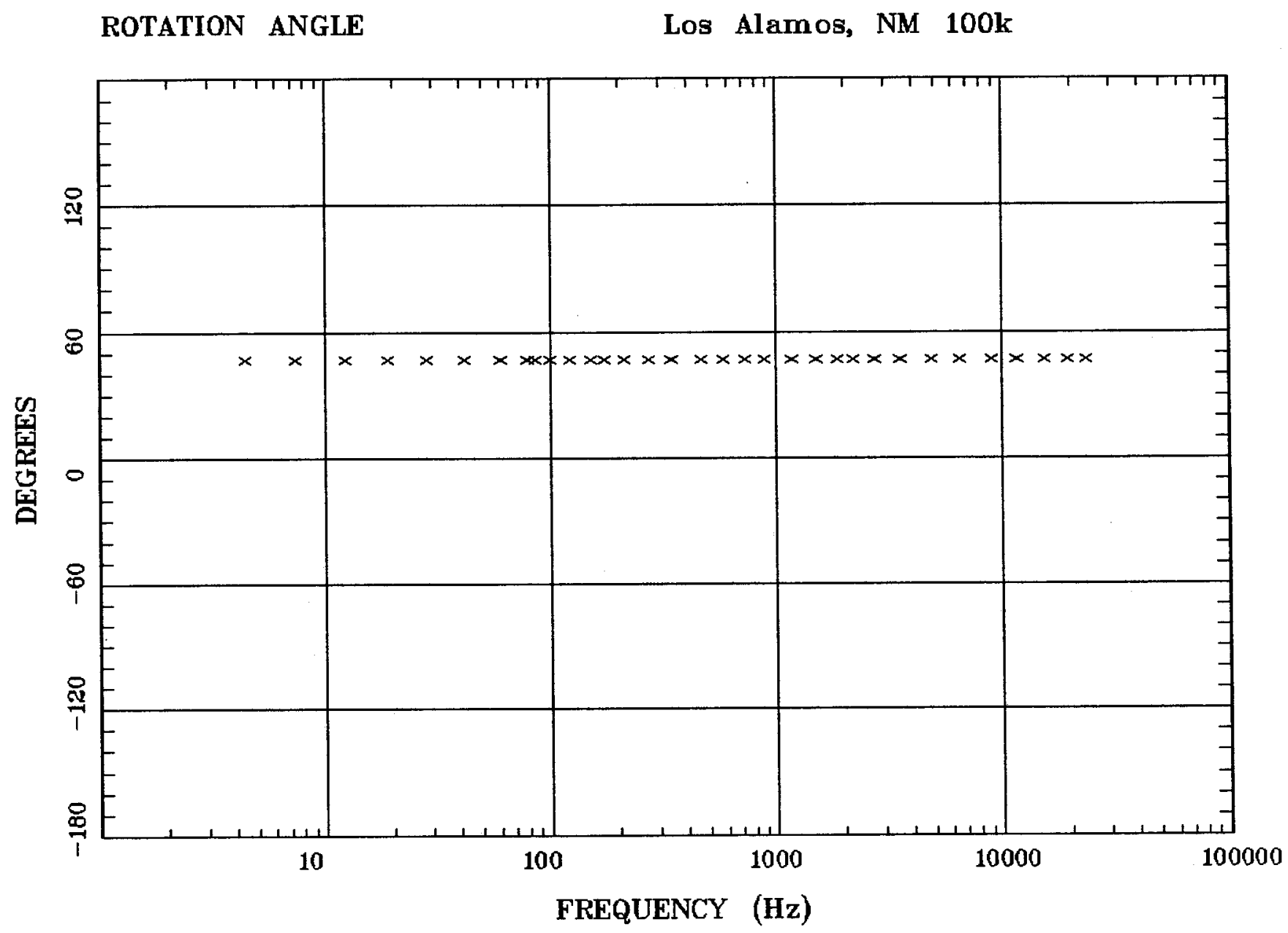

Client: Espanola Basin

Remote: none

Acquired: 14:0 Jun 04, 2004

Survey Co:USGS
Rotation:

Filename: cp30a.avg

Channels: Ch1 Ch2 Ch3 Ch4 Ch5 Ch3 Ch4 Plotted: 10:26 Aug 23, 2004

< EMI - ElectroMagnetic Instruments > 
Station 30

IMPEDANCE SKEW

Los Alamos, NM 100k

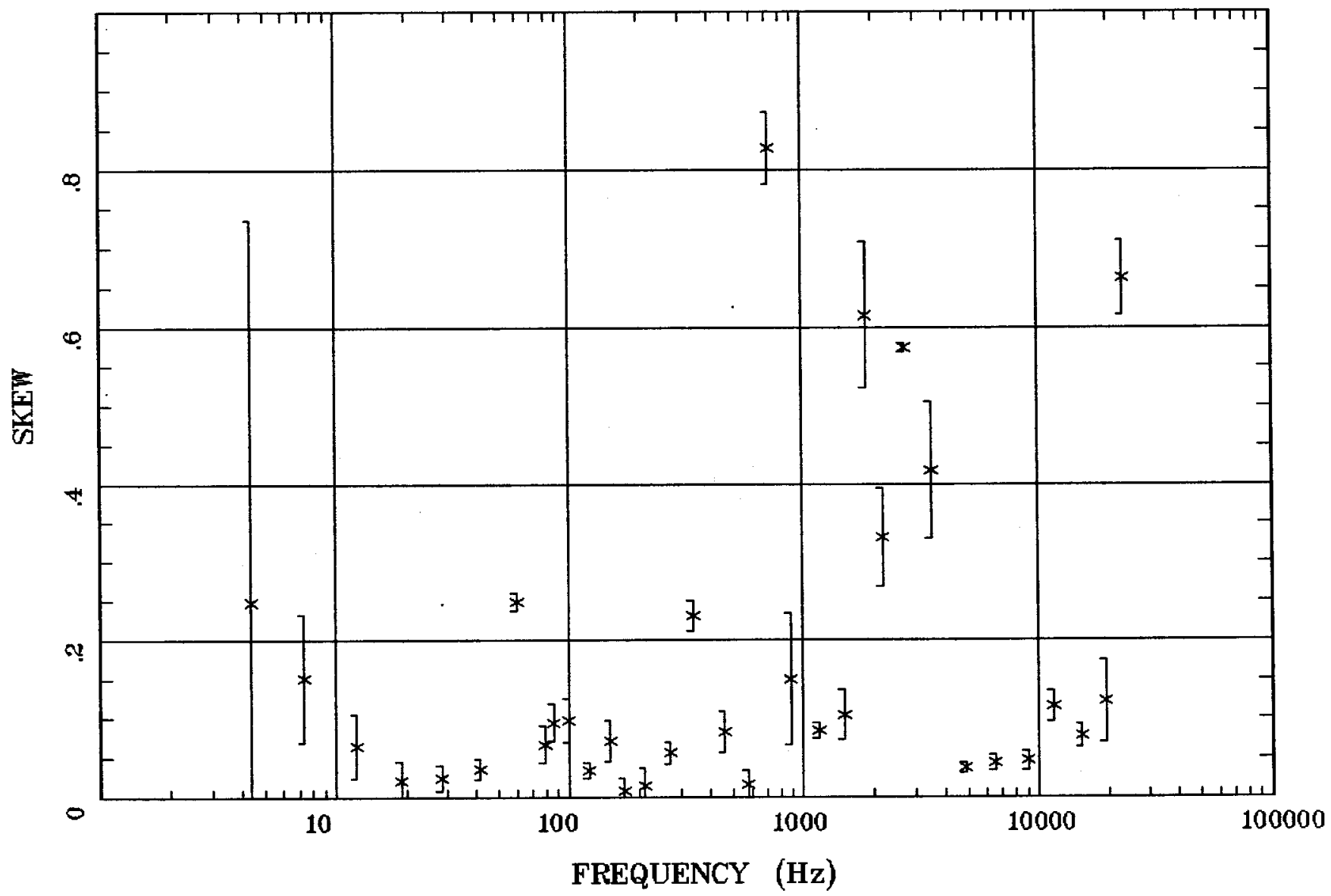

Client: Espanola Basin

Remote: nane

Acquired: 14:0 Jun 04, 2004

Survey Co:USGS
Rotation:

Filename: cp30a.avg

Channels: Ch1 Ch2 Ch3 Ch4 Ch5 Ch3 Ch4 Plotted: 10:26 Aug 23, 2004

< EMI - ElectroMagnetic Instruments > 
E MULT Coh.

Los Alamos, NM 100k

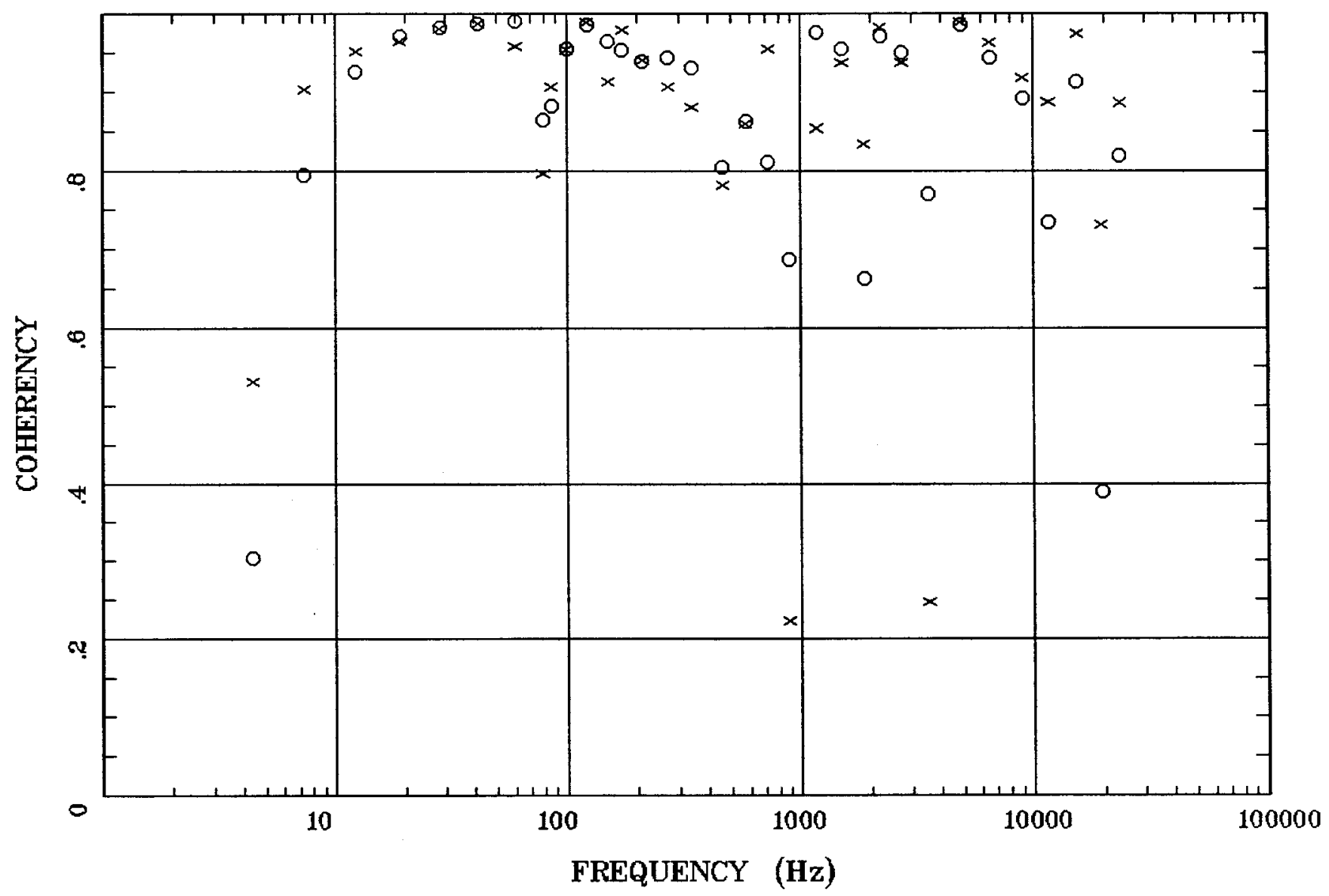

Client: Espanola Basin

Remote: none

Acquired: 14:0 Jun 04, 2004

Survey Co:USGS
Rotation:

Filename: cp30a.avg

Channels: Ch1 Ch2 Ch3 Ch4 Ch5 Ch3 Ch4

Plotted: 10:26 Aug 23, 2004

< EMI - ElectroMagnetic Instruments > 


\section{POLAR PLOTS}

Los Alamos, NM 100k

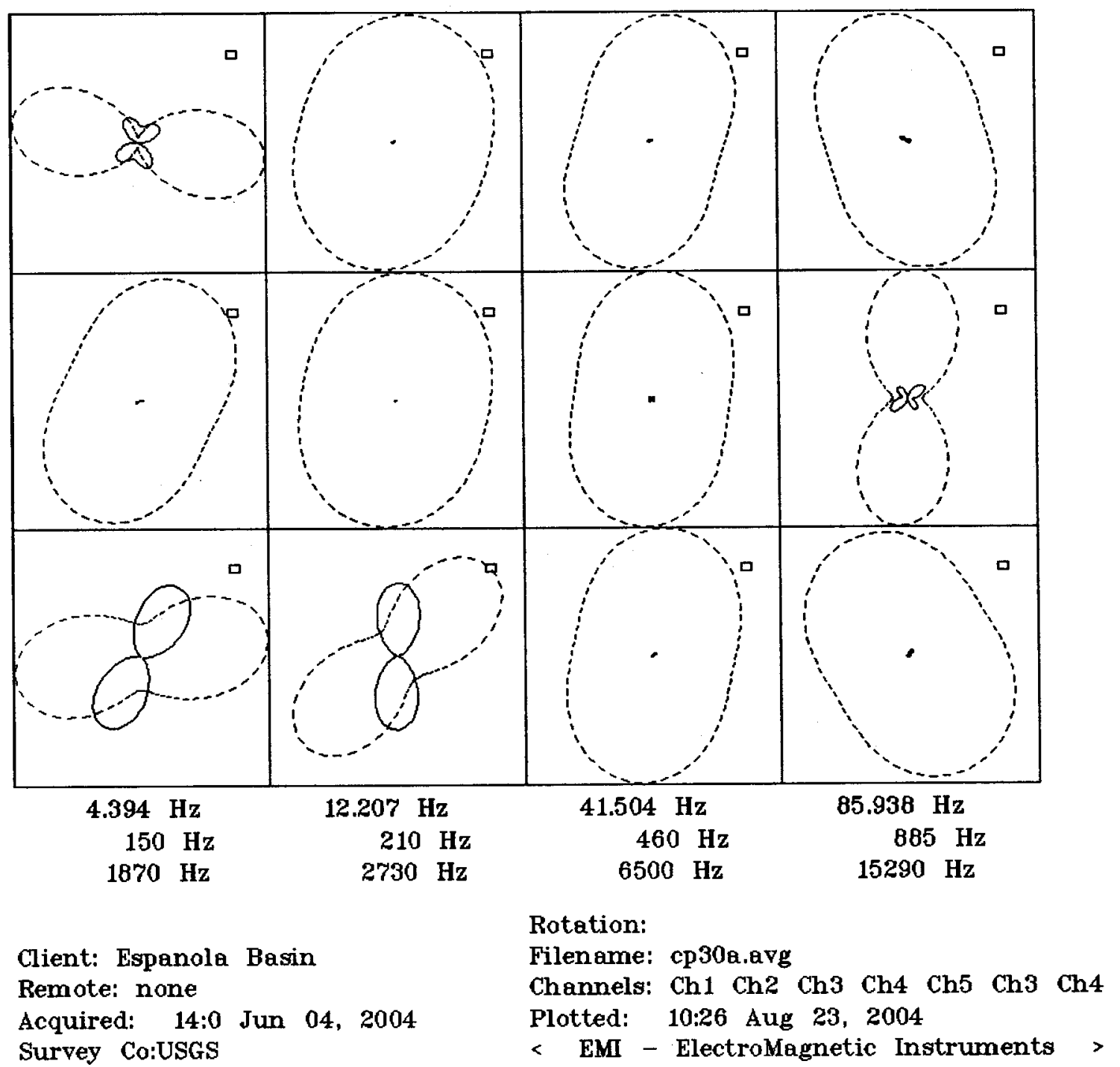




\section{Station 30}

TIPPER MAGNITUDE

Los Alamos, NM 100k

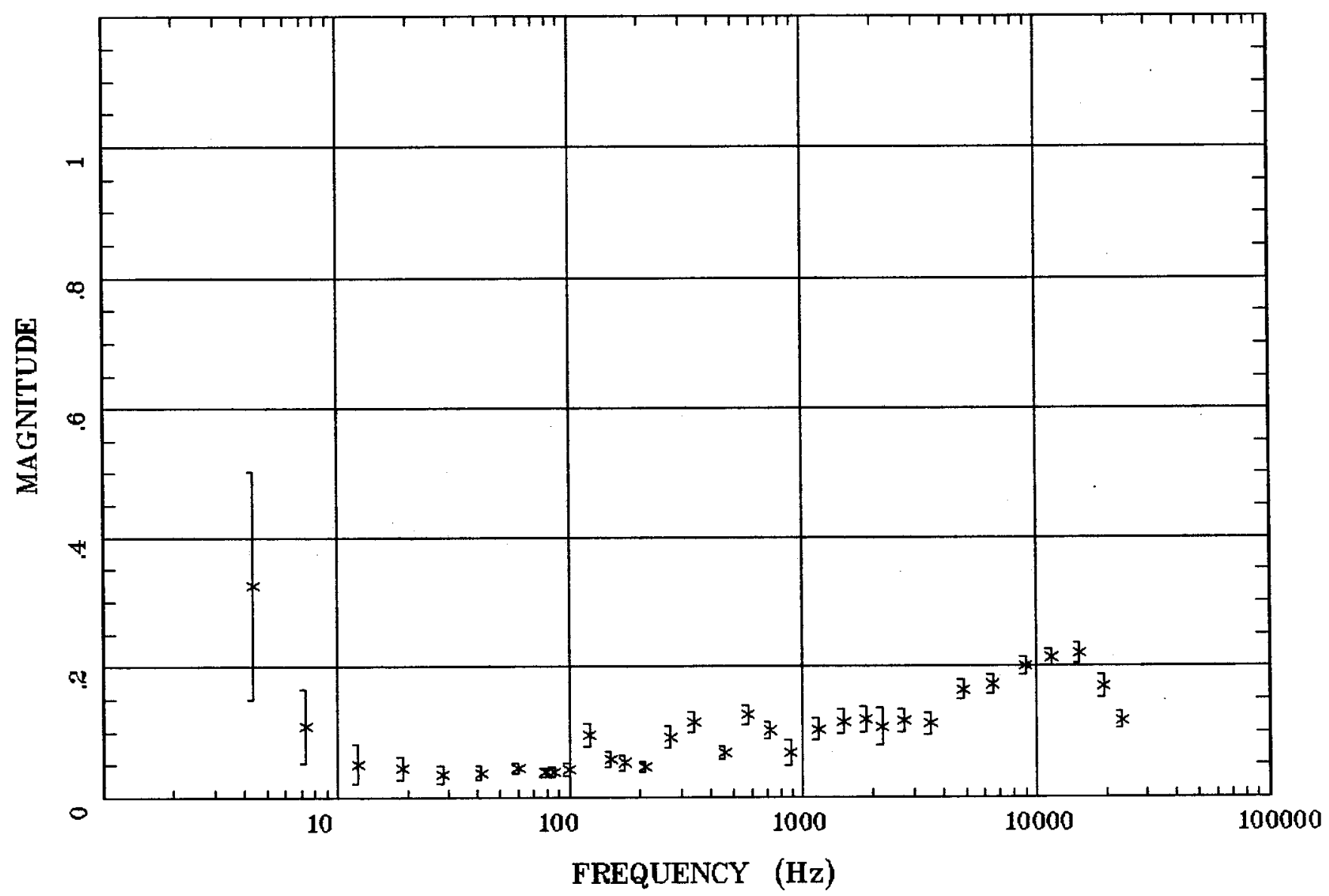

Client: Espanola Basin

Remote: none

Acquired: 14:0 Jun 04, 2004 Survey Co:USGS
Rotation:

Filename: cp30a.avg

Channels: Ch1 Ch2 Ch3 Ch4 Ch5 Ch3 Ch4 Plotted: 10:26 Aug 23, 2004

< EMI - ElectroMagnetic Instruments 
Los Alamos, NM 100k

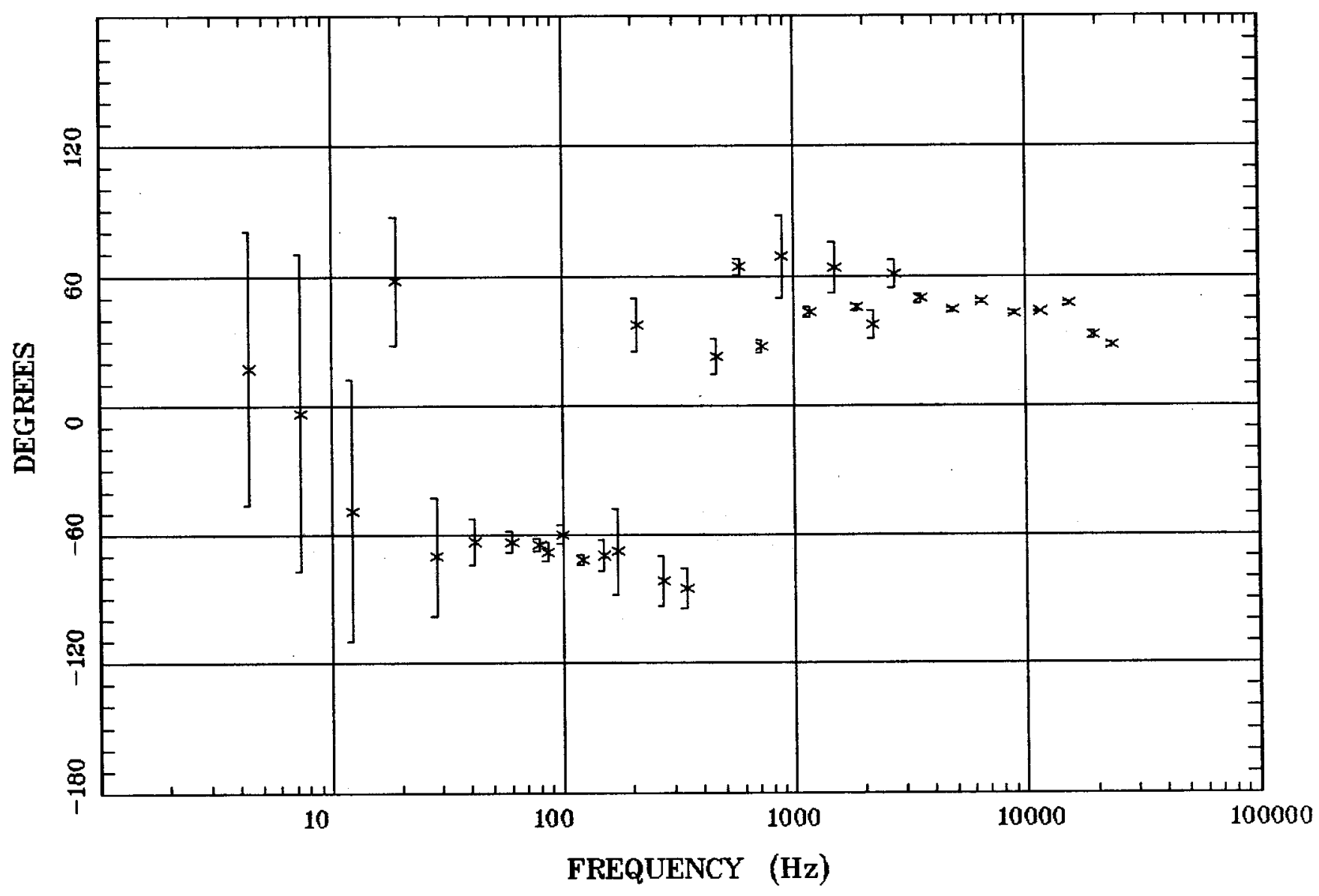

Client: Espanala Basin

Remote: none

Acquired: 14:0 Jun 04, 2004

Survey Co:USGS
Rotation:

Filename: cp30a.avg

Channels: Ch1 Ch2 Ch3 Ch4 Ch5 Ch3 Ch4

Plotted: 10:26 Aug 23, 2004

< EMI - ElectroMagnetic Instruments > 
Station 30

HzHx.x Coh HzHy.o Los Alamos, NM 100k

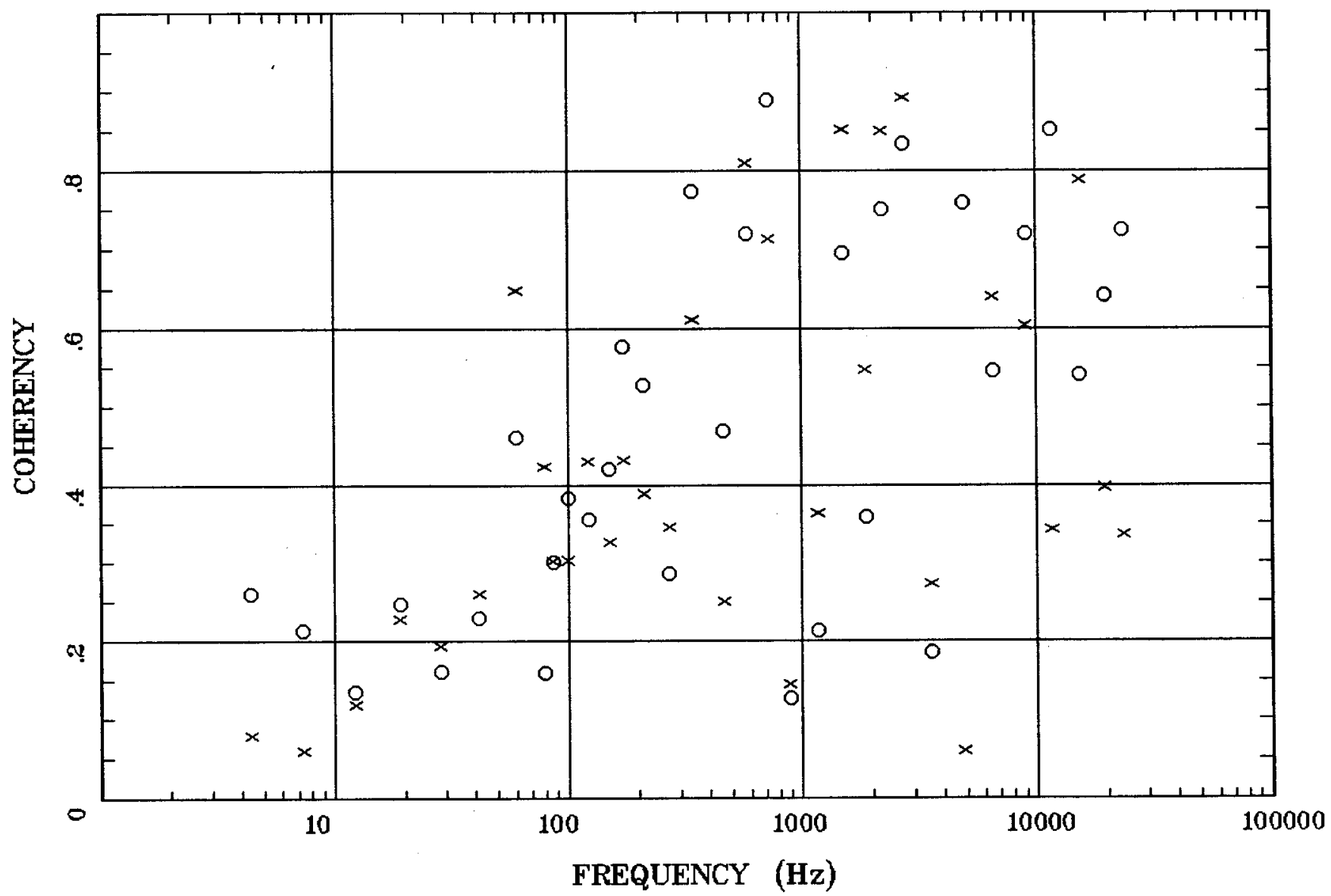

Client: Espanola Basin

Remote: none

Acquired: 14:0 Jun 04, 2004 Survey Co:USGS
Rotation:

Filename: cp30a.avg

Channels: Ch1 Ch2 Ch3 Ch4 Ch5 Ch3 Ch4 Plotted: 10:26 Aug 23, 2004

< EMI - ElectroMagnetic Instruments > 


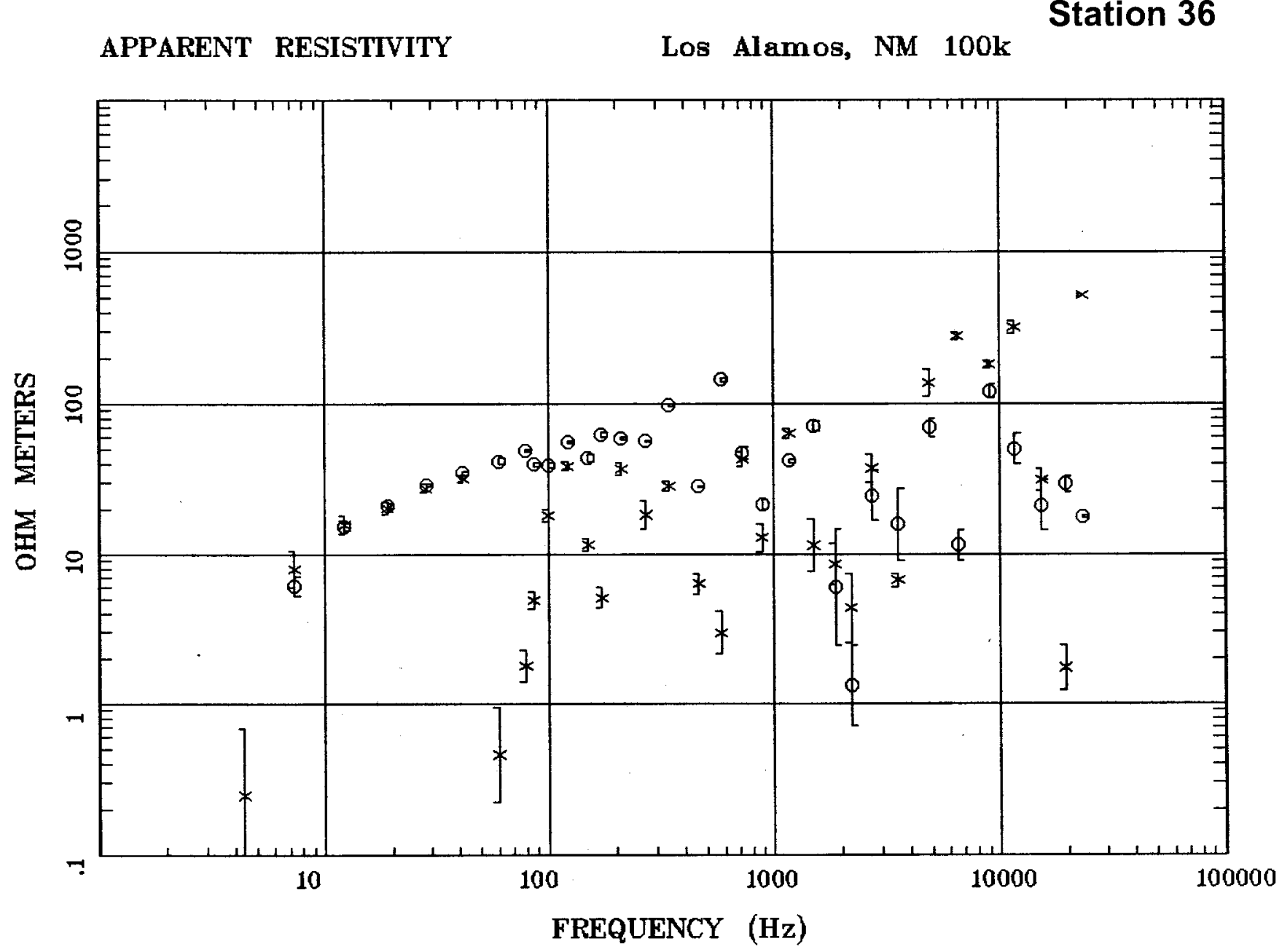

Client: Espanola Basin Remote: none Acquired: 12:1 Jun 07, 2004 Survey Co:USGS
Rotation:

Filename: cp36a.avg

Channels: Ch1 Ch2 Ch3 Ch4 Ch5 Ch3 Ch4 Plotted: 09:20 Aug 23, 2004

< EMI - ElectroMagnetic Instruments > 


\section{IMPEDANCE PHASE}

Los Alamos, NM 100k

Station 36

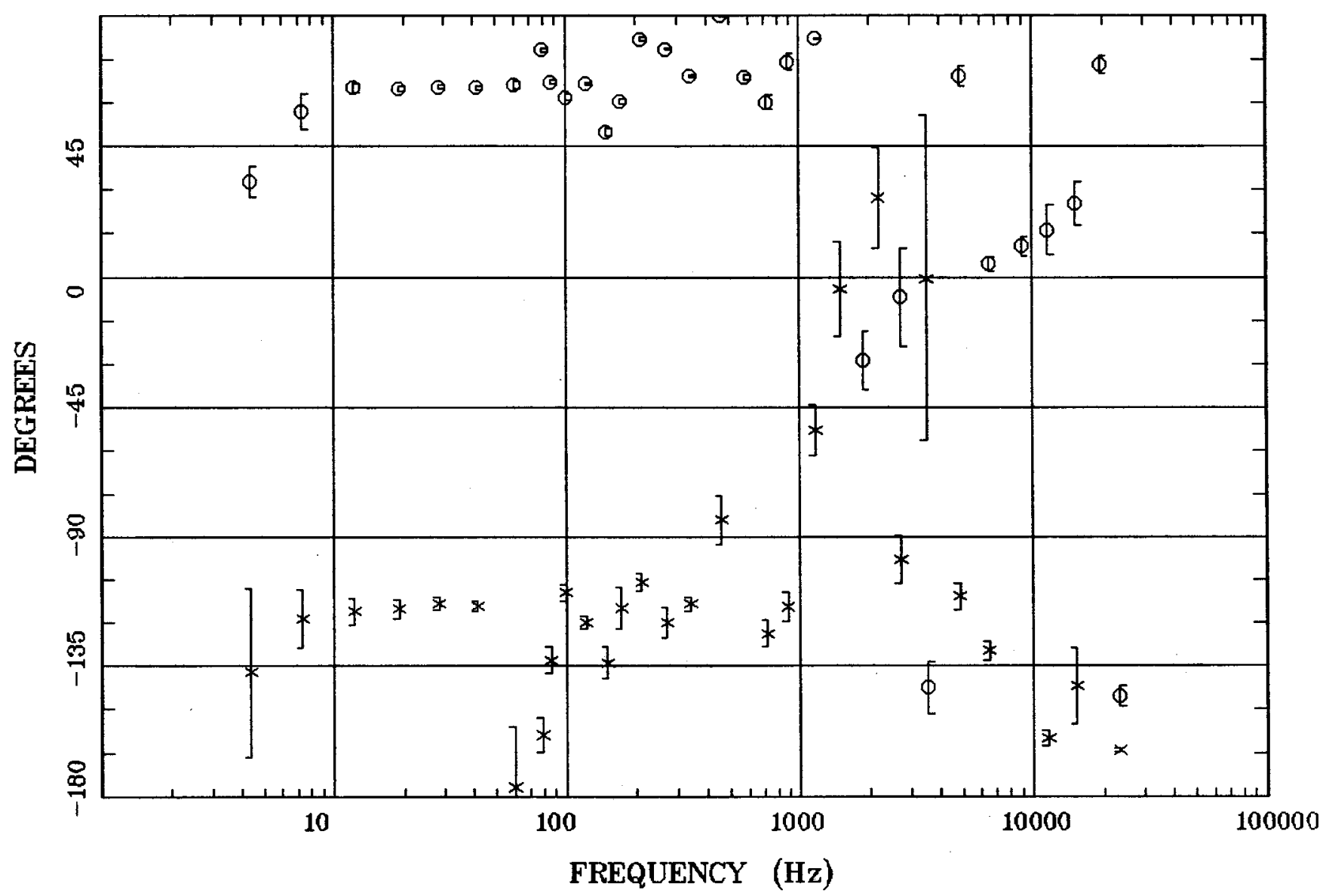

Client: Espanola Basin Remote: none

Acquired: 12:1 Jun 0\%, 2004 Survey Co:USGS
Rotation:

Filename: cp36a.avg

Channels: Ch1 Ch2 Ch3 Ch4 Ch5 Ch3 Ch4 Plotted: 09:20 Aug 23, 2004

< EMI - ElectroMagnetic Instruments > 


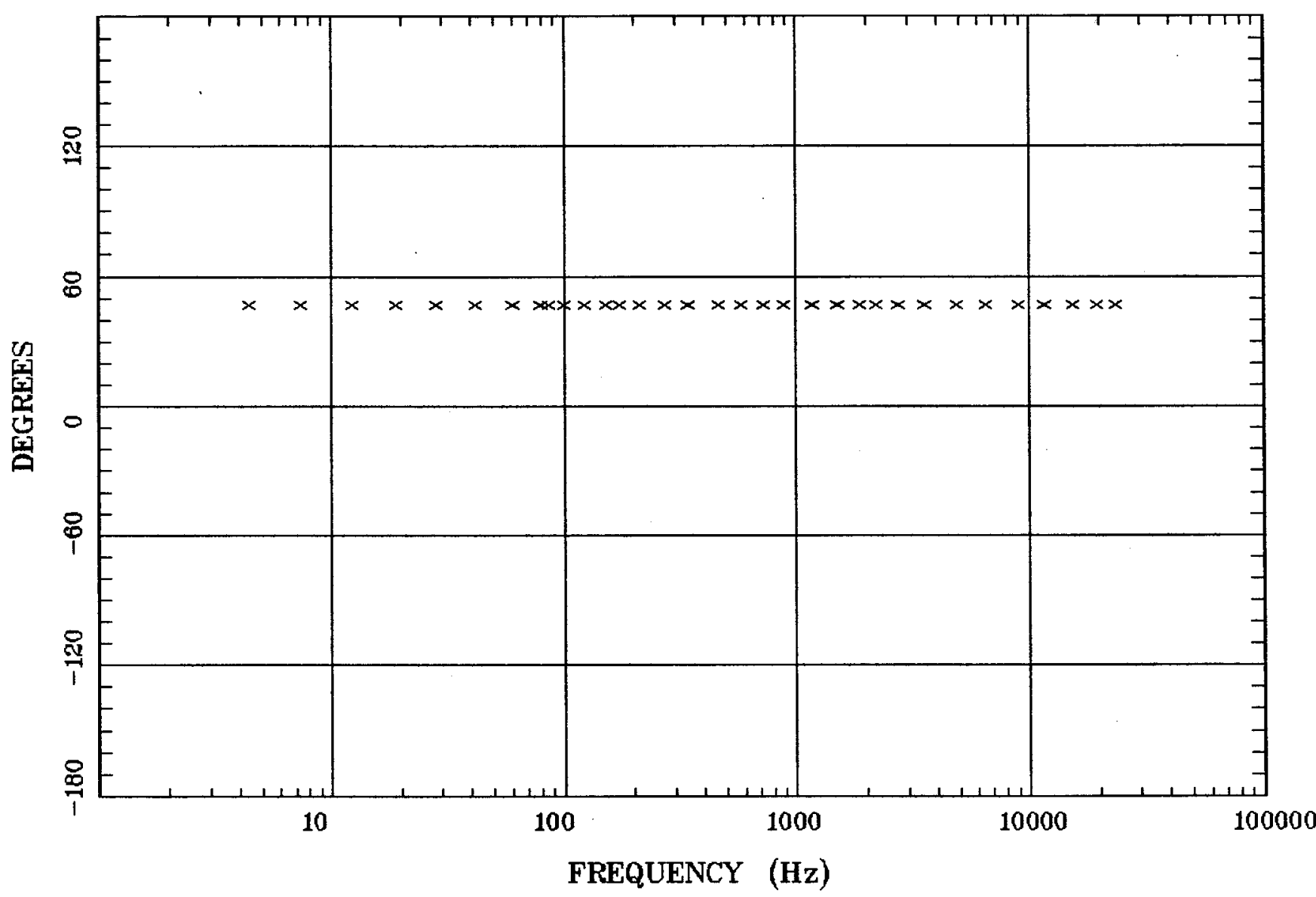

Client: Espanola Basin

Remote: none

Acquired: 12:1 Jun 07, 2004

Survey Co:USGS
Rotation:

Filename: cp36a.arg

Channels: Ch1 Ch2 Ch3 Ch4 Ch5 Ch3 Ch4 Plotted: 09:20 Aug 23, 2004

$<$ EMI - ElectroMagnetic Instruments 


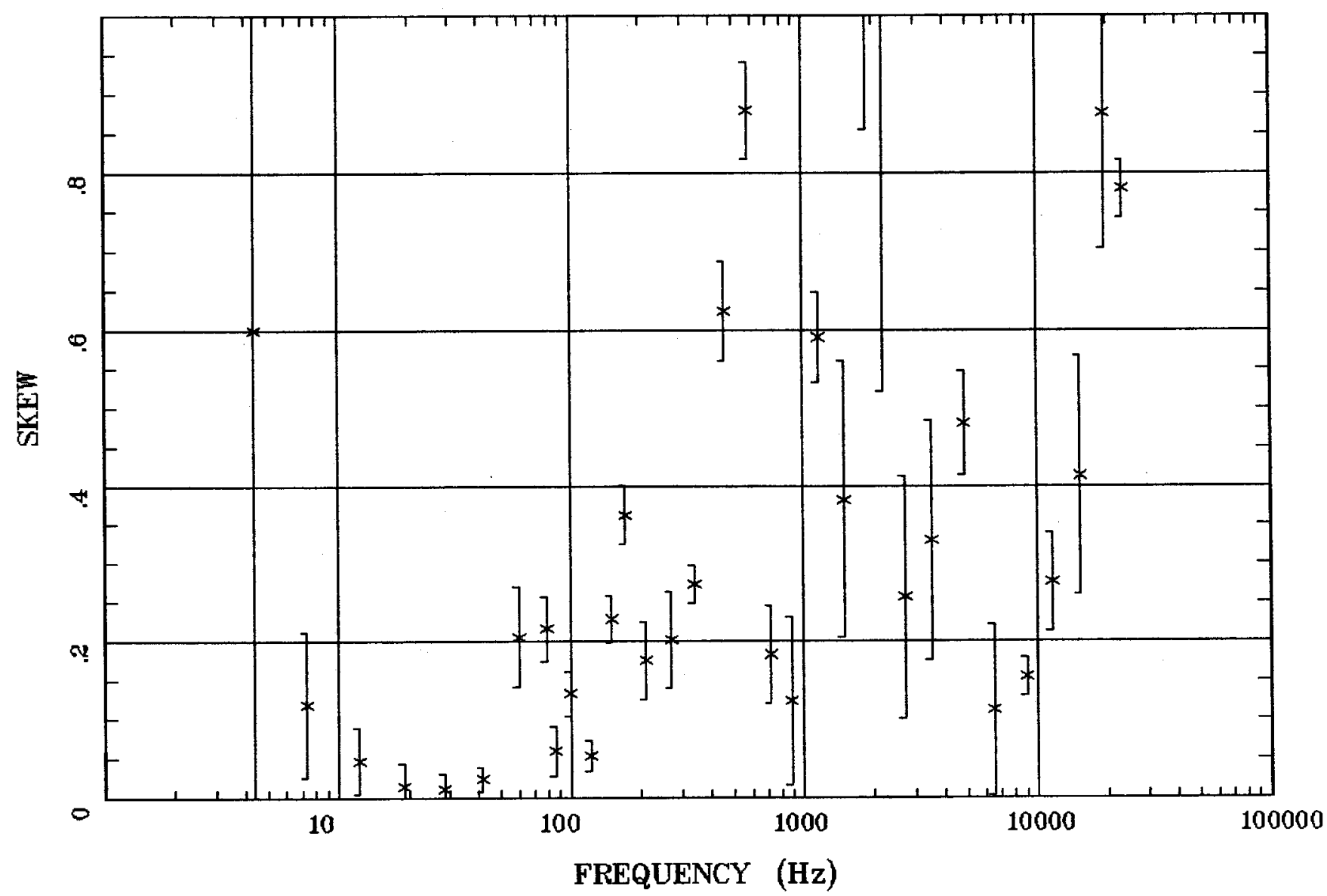

Client: Espanola Basin

Remote: none

Acquired: 12:1 Jun 07, 2004 Survey Co:USGS
Rotation:

Filename: cp36a.avg

Channels: Ch1 Ch2 Ch3 Ch4 Ch5 Ch3 Ch4 Plotted: 09:20 Aug 23, 2004

< EMI - ElectroMagnetic Instruments 


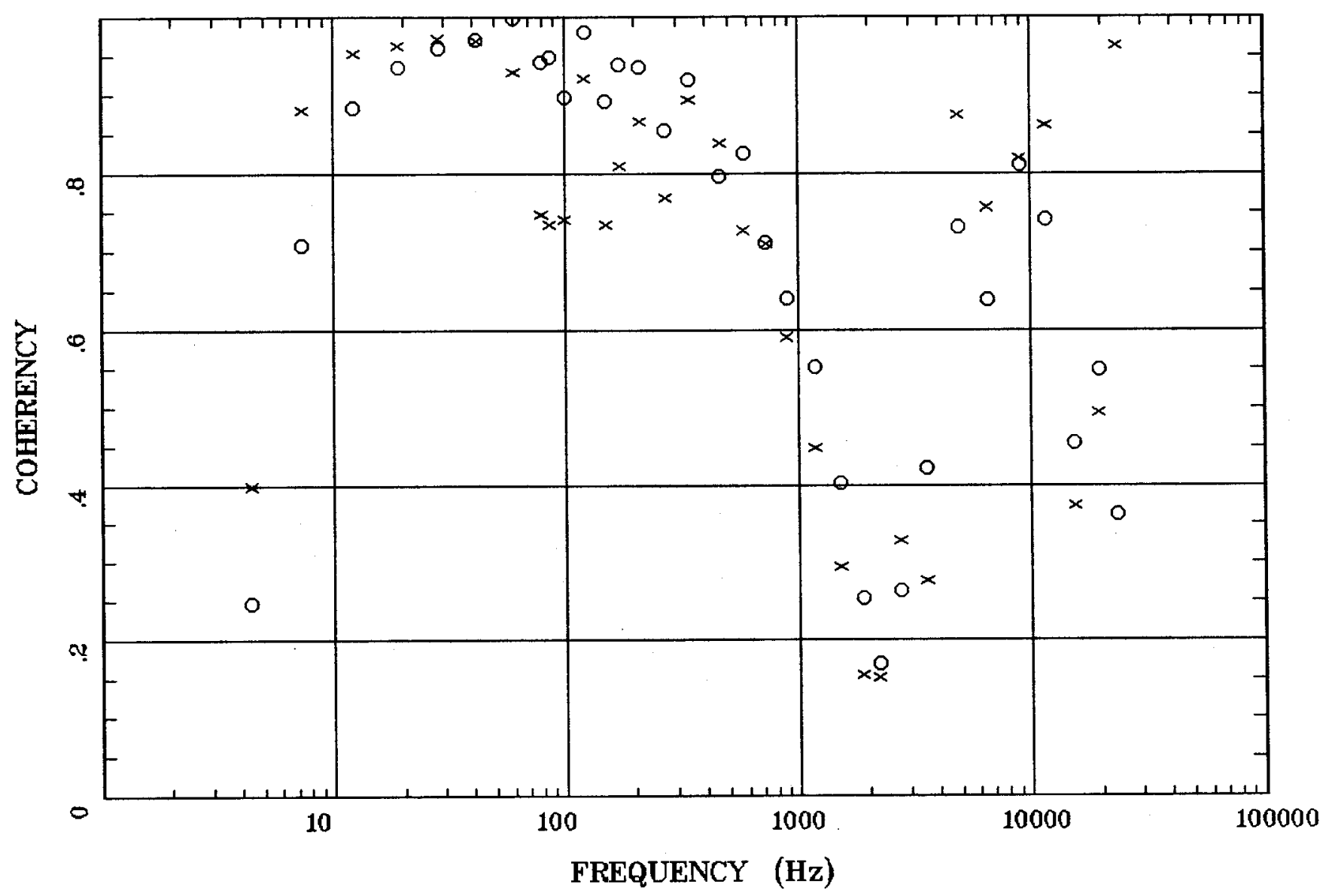

Client: Espanola Basin

Remote: none

Acquired: 12:1 Jun 07, 2004

Survey Co:USGS
Rotation:

Filename: cp36a.avg

Channels: Ch1 Ch2 Ch3 Ch4 Ch5 Ch3 Ch4 Plotted: 09:20 Aug 23, 2004

< EMI - ElectroMagnetic Instruments > 


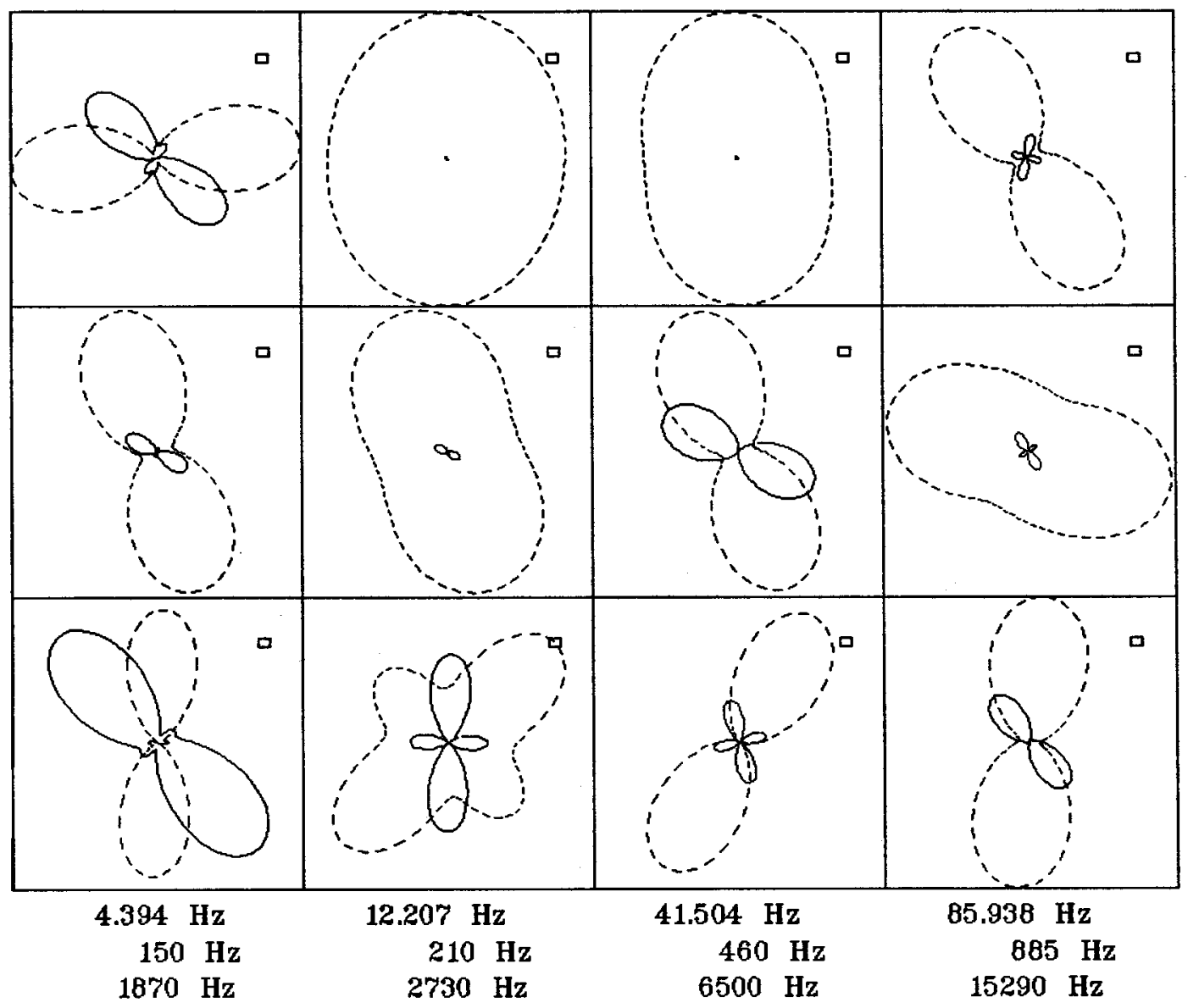

Client: Espanola Basin

Remote: none

Acquired: 12:1 Jun 07, 2004 Survey Co:USGS
Rotation:

Filename: cp36a.avg

Channels: Ch1 Ch2 Ch3 Ch4 Ch5 Ch3 Ch4 Plotted: 09:20 Aug 23, 2004

$<$ EMI - ElectroMagnetic Instruments 


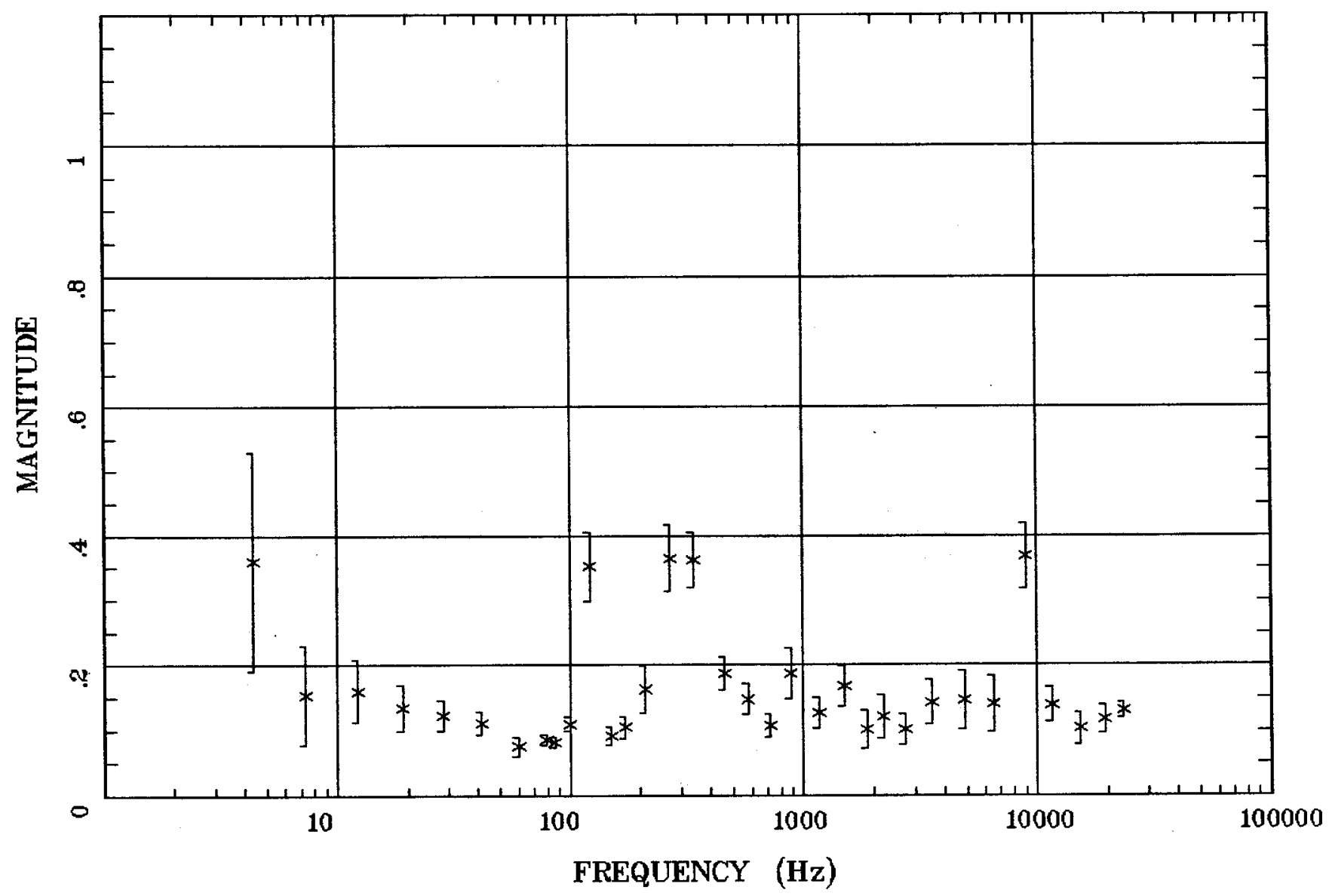

Client: Espanola Basin

Remote: none

Acquired: 12:1 Jun 07, 2004 Survey Co:USGS
Rotation:

Filename: cp36a.avg

Channels: Ch1 Ch2 Ch3 Ch4 Ch5 Ch3 Ch4 Plotted: 09:20 Aug 23, 2004

< EMI - ElectroMagnetic Instruments 


\section{TIPPER STRIKE}

Los Alamos, NM 100k

Station 36

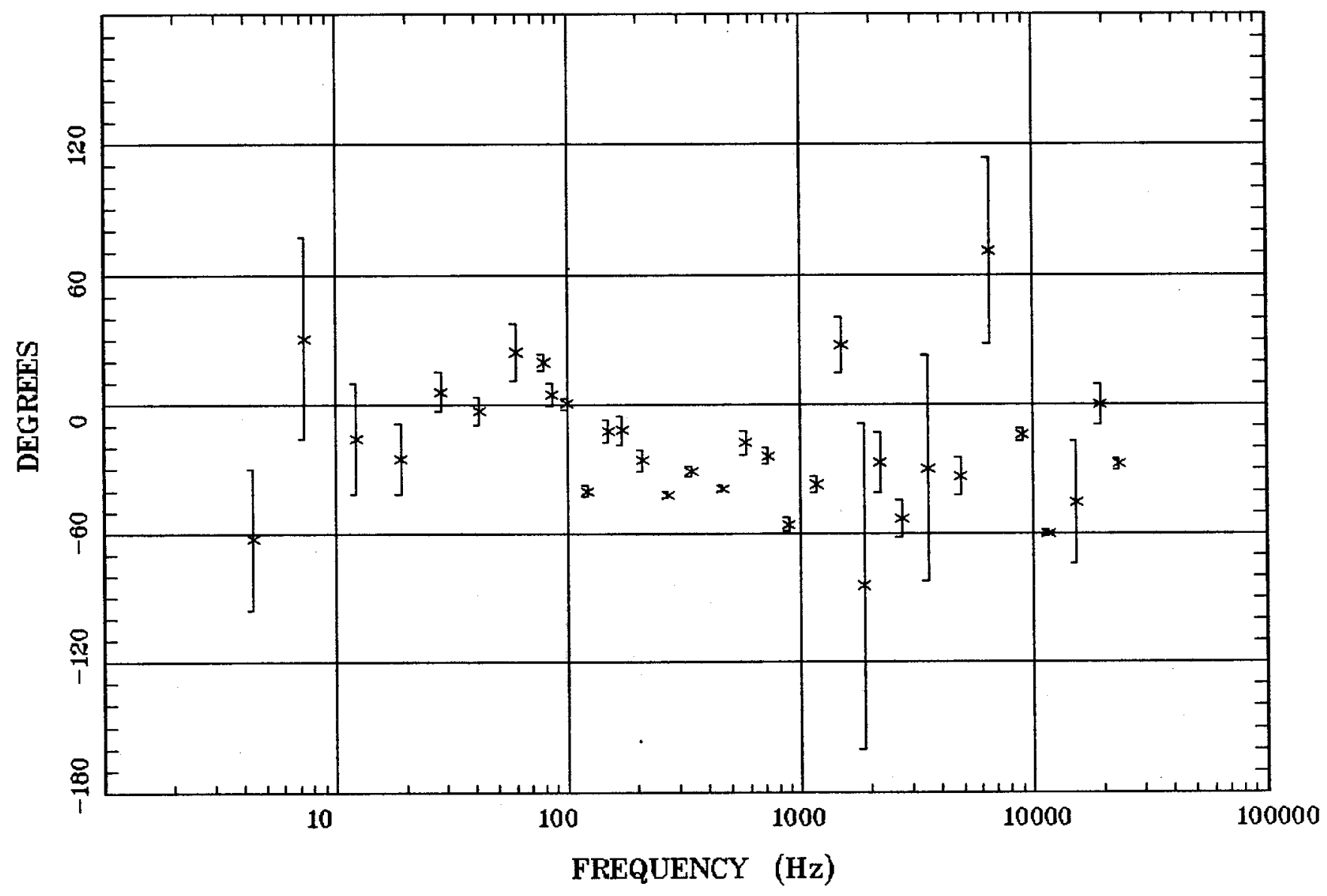

Client: Espanola Basin

Remote: none

Acquired: 12:1 Jun 07, 2004

Survey Co:USGS
Rotation:

Filename: cp36a.avg

Channels: Ch1 Ch2 Ch3 Ch4 Ch5 Ch3 Ch4

Plotted: 09:20 Aug 23, 2004

< EMI - ElectroMagnetic Instruments > 
HzHx.x Coh HzHy.o

Los Alamos, NM 100k

Station 36

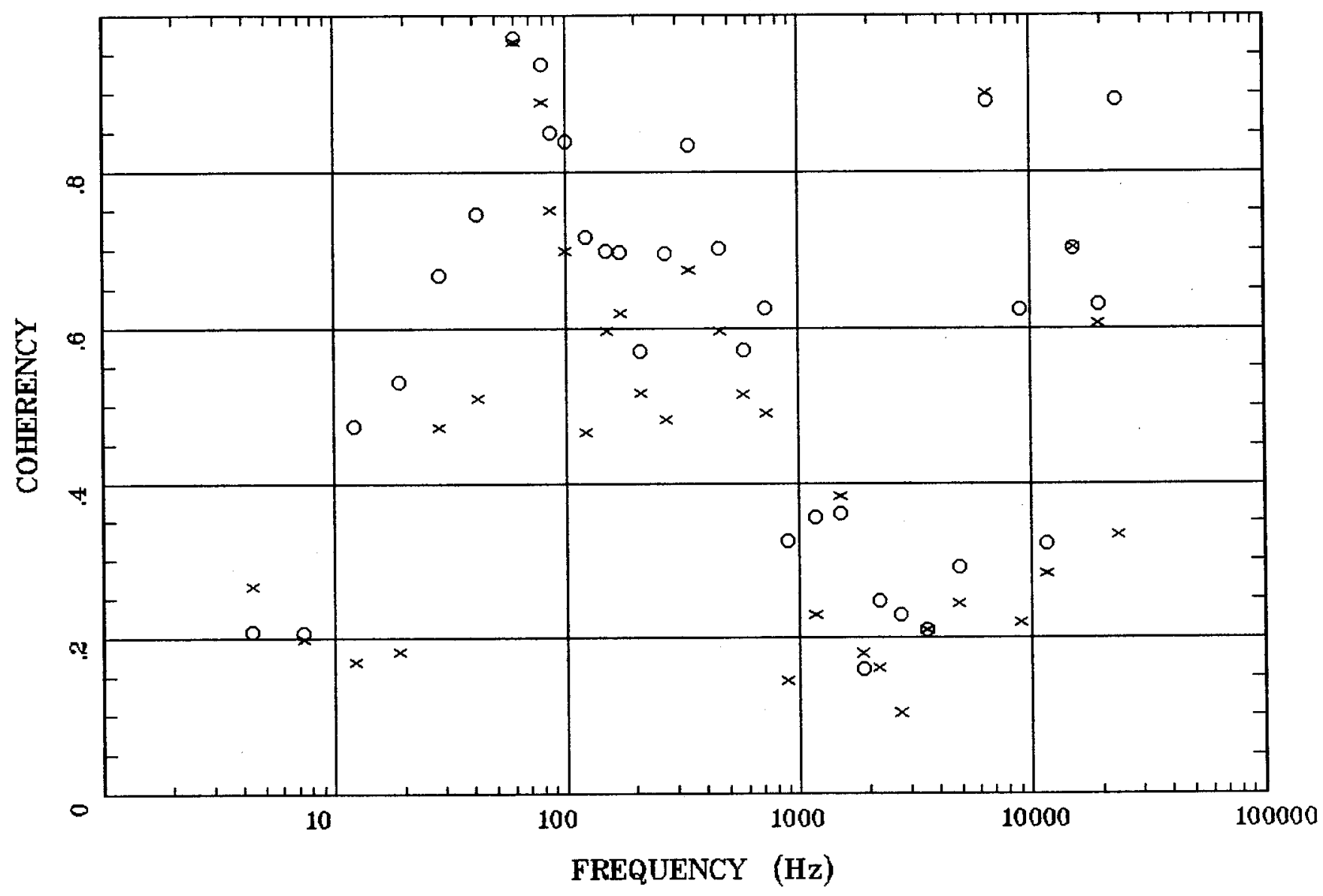

Client: Espanola Basin

Remote: none

Acquired: 12:1 Jun 07, 2004

Survey co:USGS
Rotation:

Filename: cp36a.avg

Channels: Ch1 Ch2 Ch3 Ch4 Ch5 Ch3 Ch4

Plotted: 09:20 Aug 23, 2004

\& EMI - ElectroMagnetic Instruments > 
APPARENT RESISTIVITY

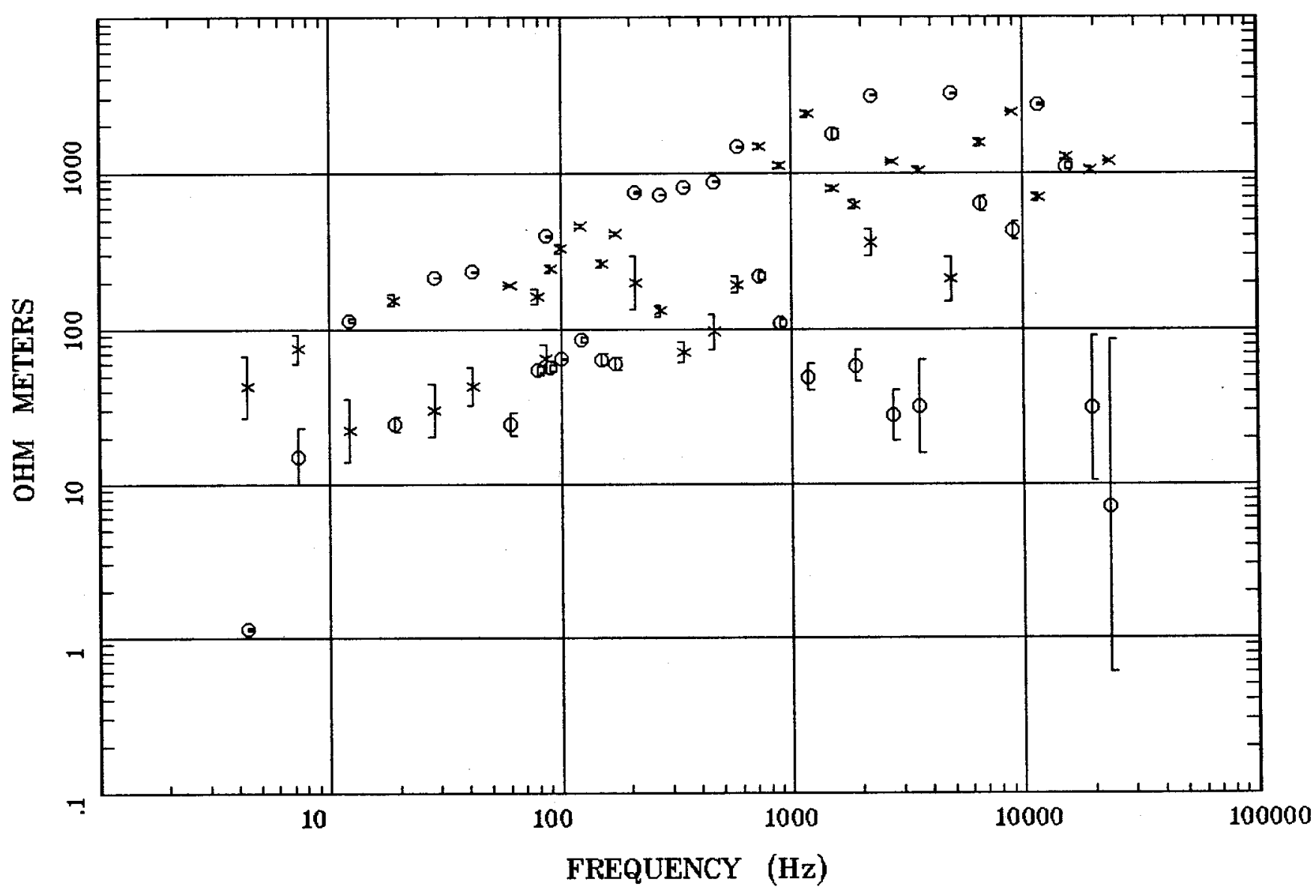

Client: Espanola Basin

Remote: none

Acquired: 12:2 Jun 03, 2004

Survey Co:USGS

Los Alamos, NM 100k

Station 15

Rotation:

Filename: cp 15aall.avg

Channels: Ch1 Ch2 Ch3 Ch4 Ch5 Ch3 Ch4

Plotted: 09:30 Aug 23, 2004

< EMI - ElectroMagnetic Instruments > 


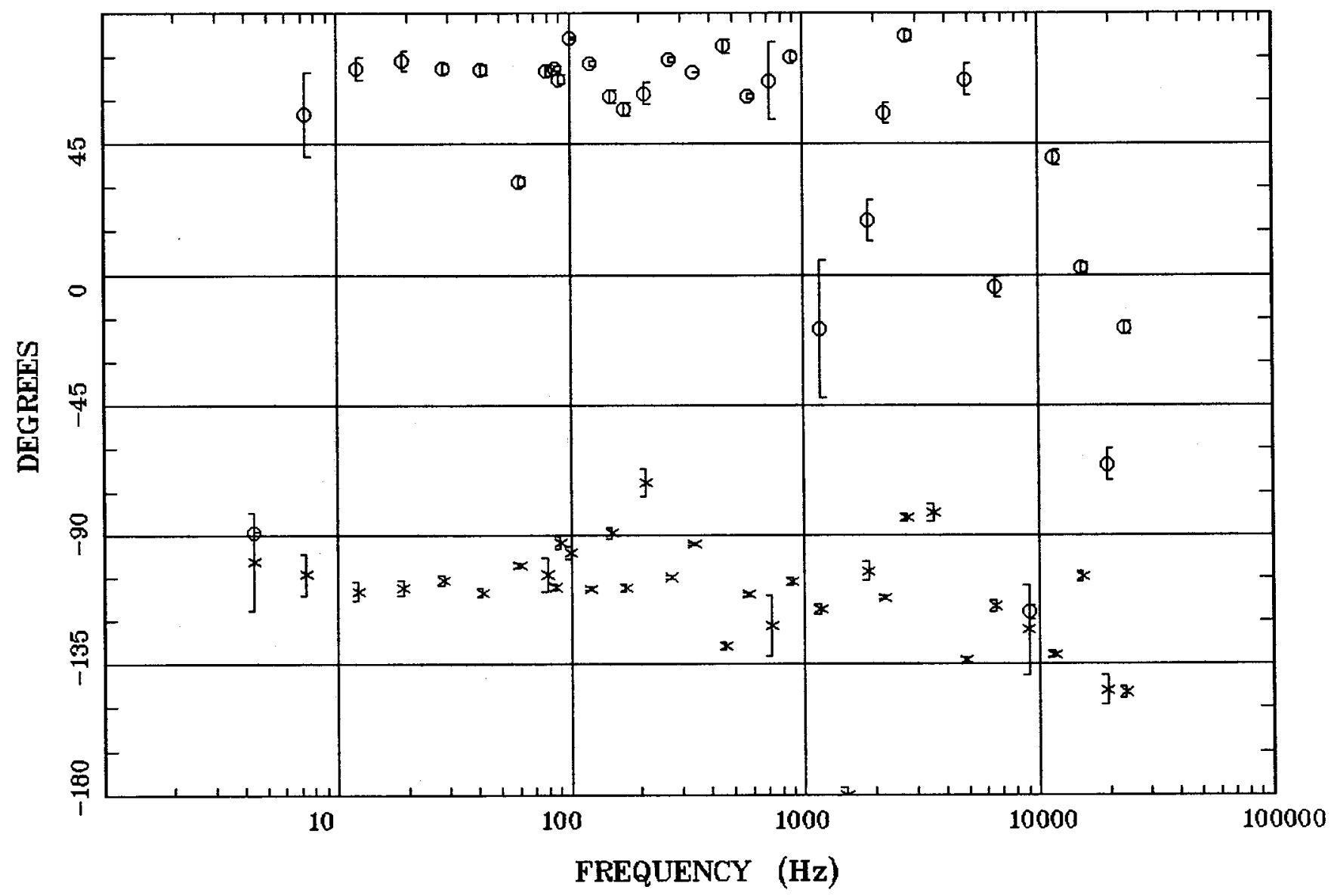

Client: Espanola Basin

Remote: none

Acquired: 12:2 Jun 03, 2004

Survey Co:USGS
Rotation:

Filename: cp 15aall.avg

Channels: Ch1 Ch2 Ch3 Ch4 Ch5 Ch3 Ch4

Plotted: 09:30 Aug 23, 2004

< EMI - ElectroMagnetic Instruments 


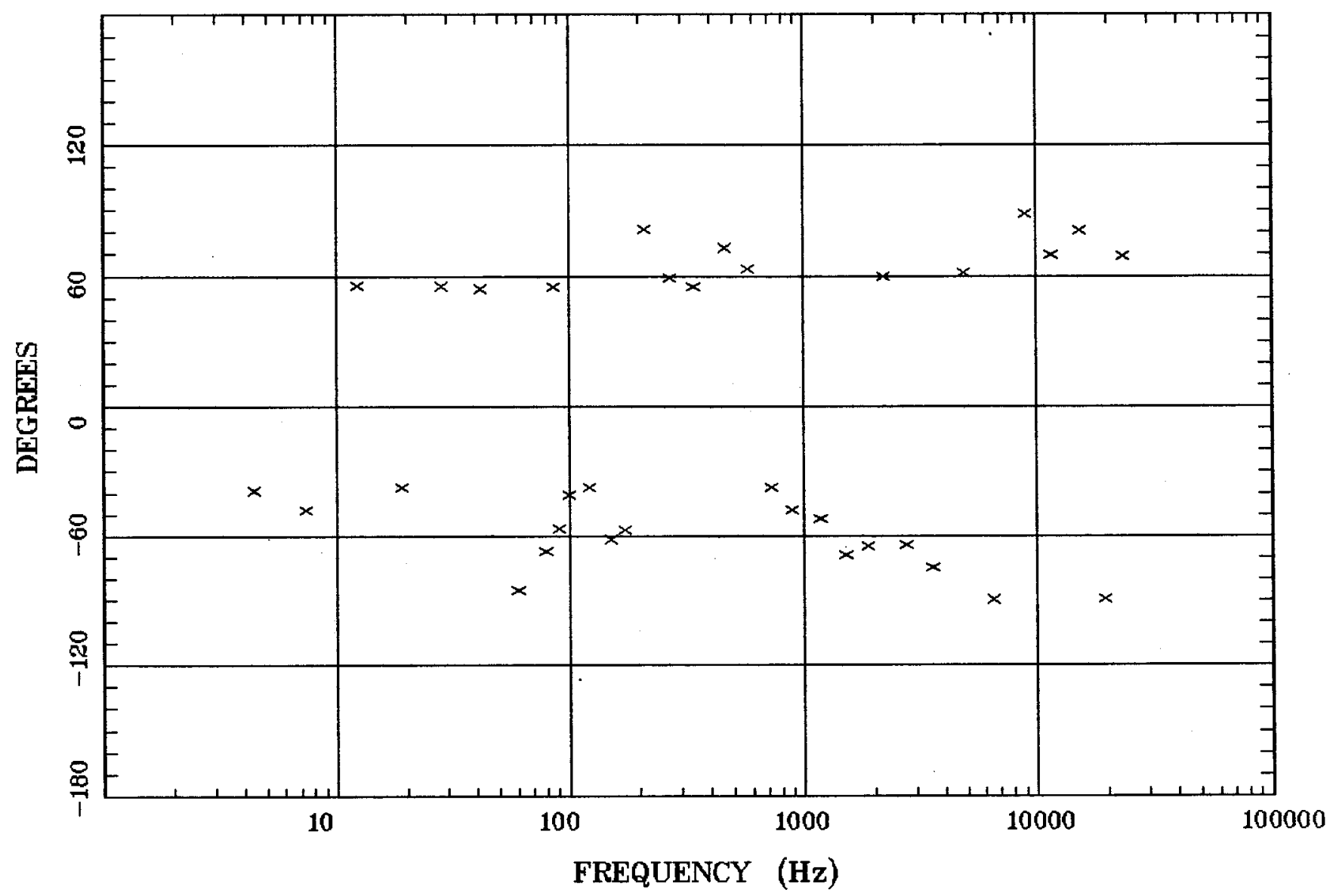

Client: Espanola Basin

Remote: none

Acquired: 12:2 Jun 03, 2004

Survey Co:USGS
Rotation:

Filename: cp 15aall.avg

Channels: Ch1 Ch2 Ch3 Ch4 Ch5 Ch3 Ch4

Platted: 09:31 Aug 23, 2004

< EMI - ElectroMagnetic Instruments > 


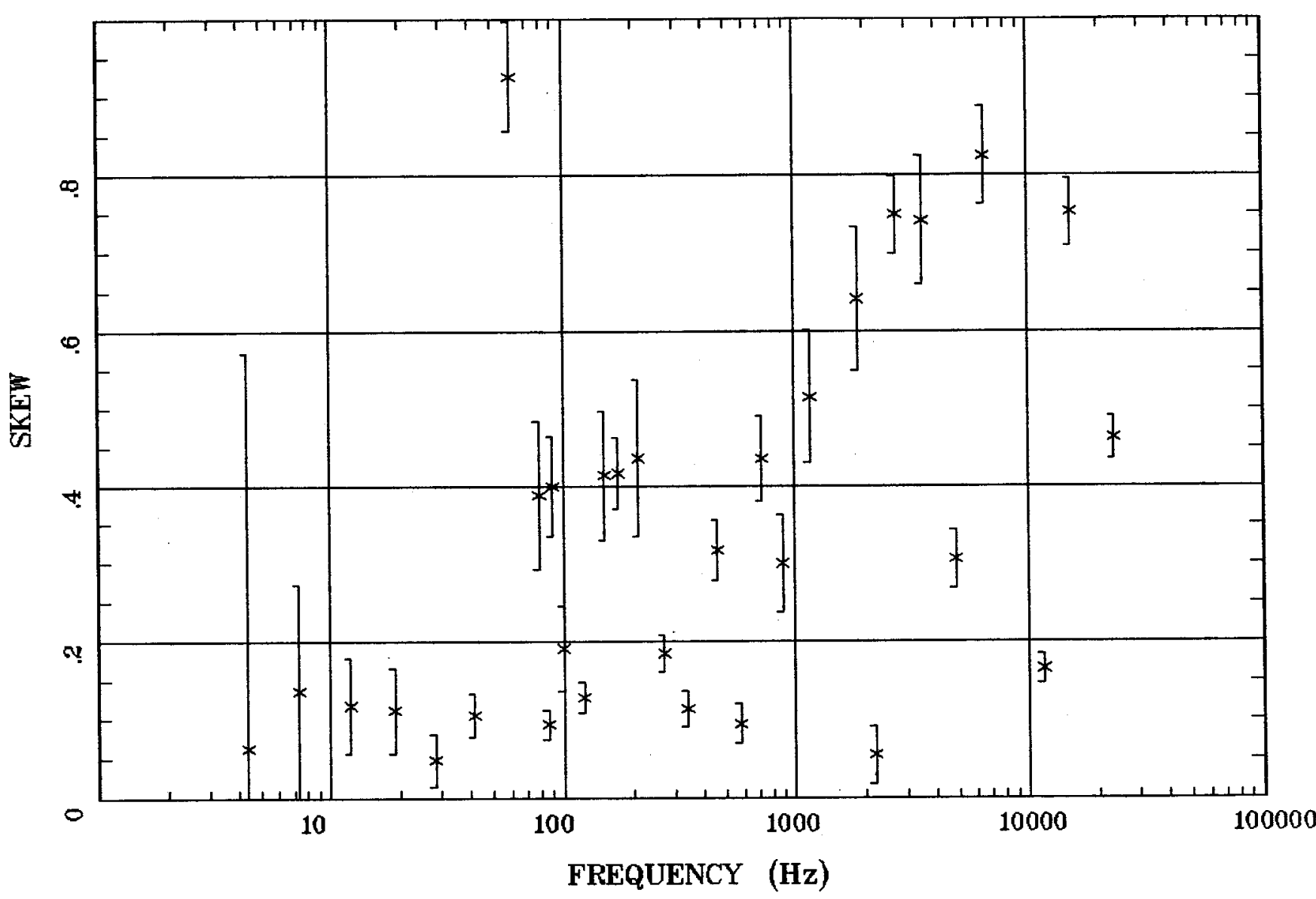

Client: Espanala Basin

Remote: none

Acquired: 12:2 Jun 03, 2004

Survey Co:USGS
Rotation:

Filename: cp 15aall.avg

Channels: Ch1 Ch2 Ch3 Ch4 Ch5 Ch3 Ch4 Plotted: 09:31 Aug 23, 2004

< EMI - ElectroMagnetic Instruments > 


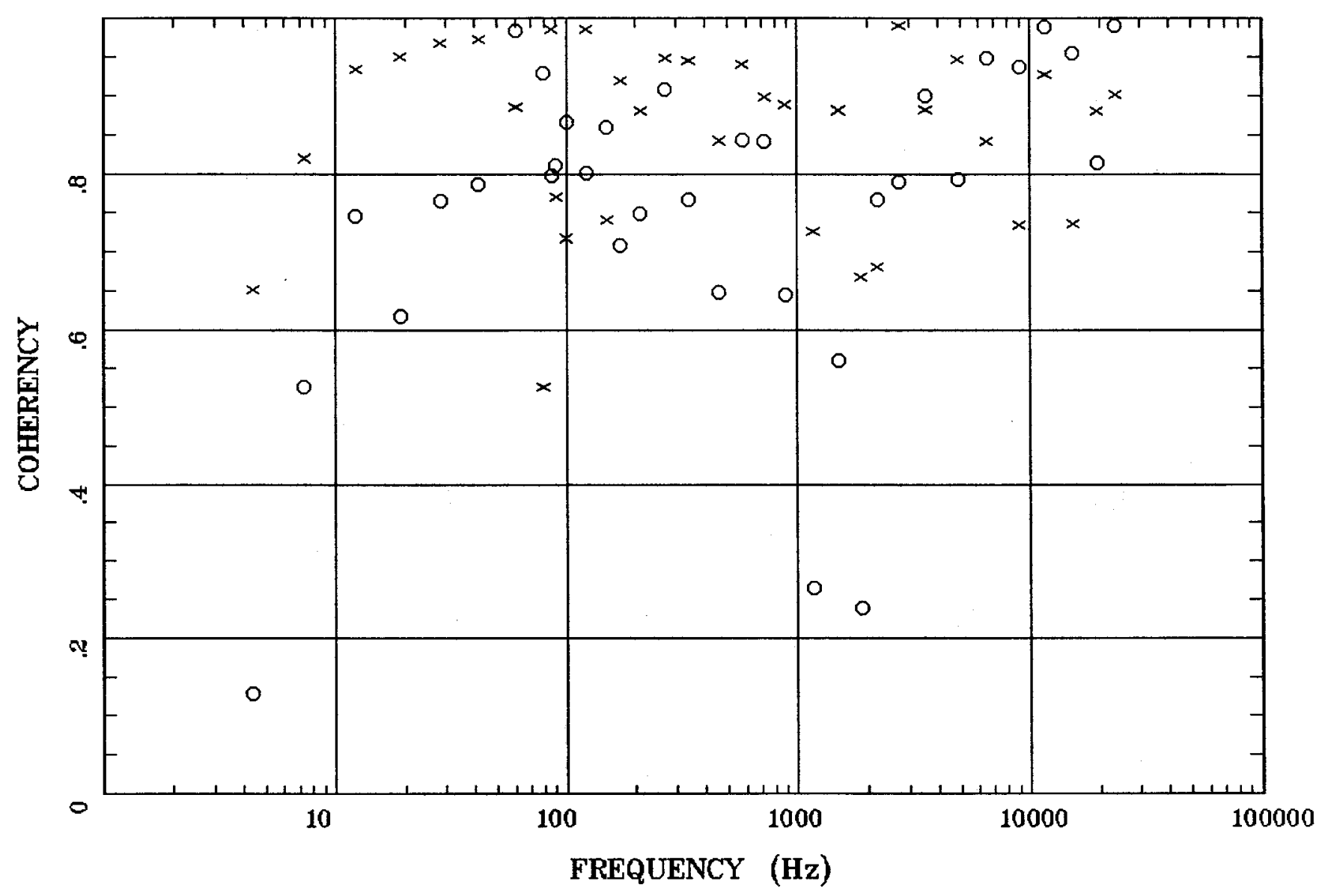

Client: Espanola Basin Remote: none Acquired: 12:2 Jun 03, 2004 Survey Co:USGS
Rotation:

Filename: cp15aall.avg

Channels: Ch1 Ch2 Ch3 Ch4 Ch5 Ch3 Ch4 Plotted: 09:31 Aug 23, 2004

$<$ EMI - ElectroMagnetic Instruments > 


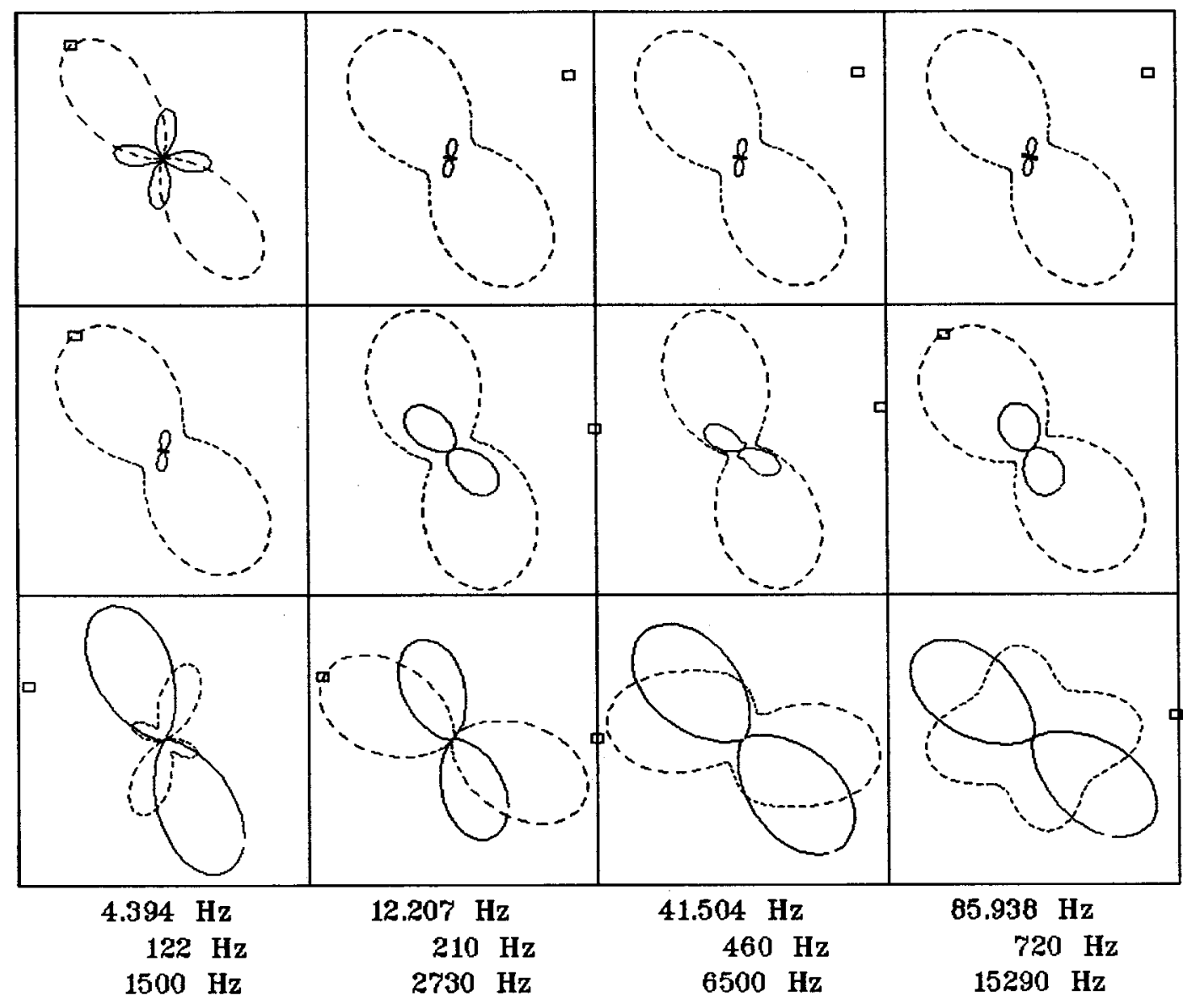

Client: Espanola Basin

Remote: none

Acquired: 12:2 Jun 03, 2004 Survey Co:USGS
Rotation:

Filename: cp15aall.avg

Channels: Ch1 Ch2 Ch3 Ch4 Ch5 Ch3 Ch4

Plotted: 09:31 Aug 23, 2004

< EMI - ElectroMagnetic Instruments 
TIPPER MAGNITUDE

Los Alamos, NM 100k

Station 15

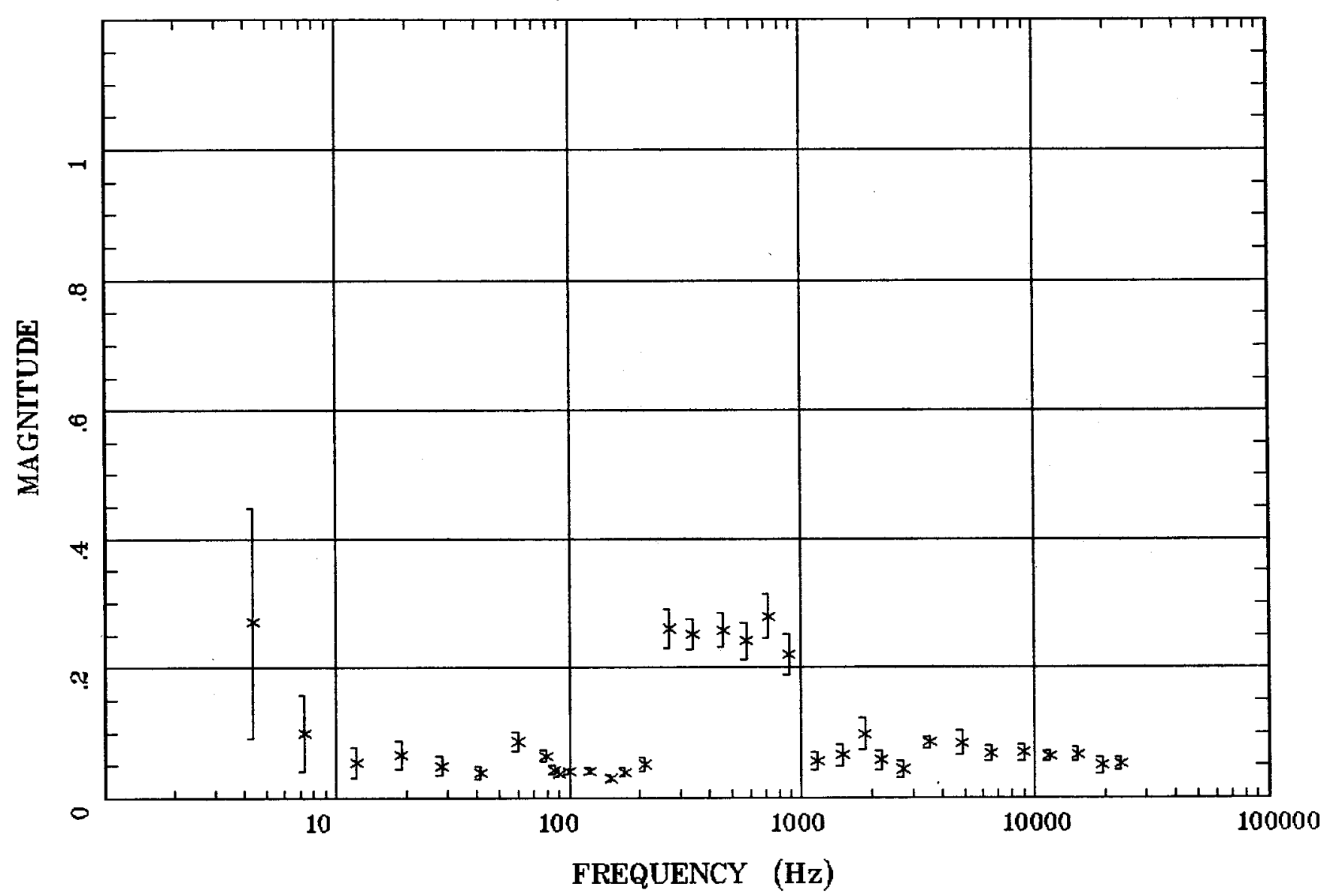

Client: Espanola Basin

Remote: none

Acquired: 12:2 Jun 03, 2004 Survey Co:USGS
Rotation:

Filename: cp15aall.avg

Channels: Ch1 Ch2 Ch3 Ch4 Ch5 Ch3 Ch4 Plotted: 09:31 Aug 23, 2004

< EMI - ElectroMagnetic Instruments 


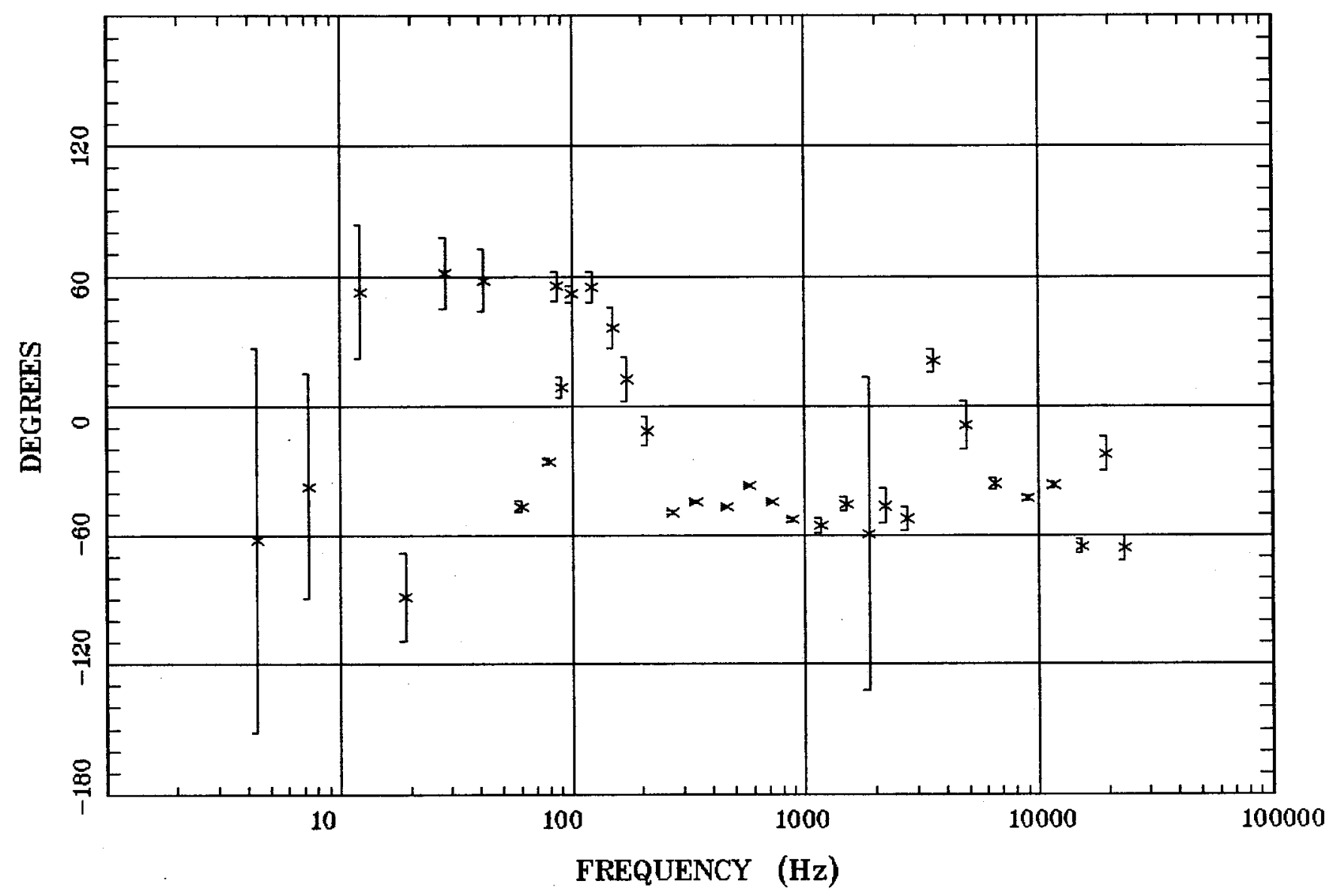

Client: Espanola Basin Remote: none Acquired: 12:2 Jun 03, 2004 Survey Co:USGS
Rotation:

Filename: cp 15aall.avg

Channels: Ch1 Ch2 Ch3 Ch4 Ch5 Ch3 Ch4

Plotted: 09:31 Aug 23, 2004

< EMI - ElectroMagnetic Instruments > 


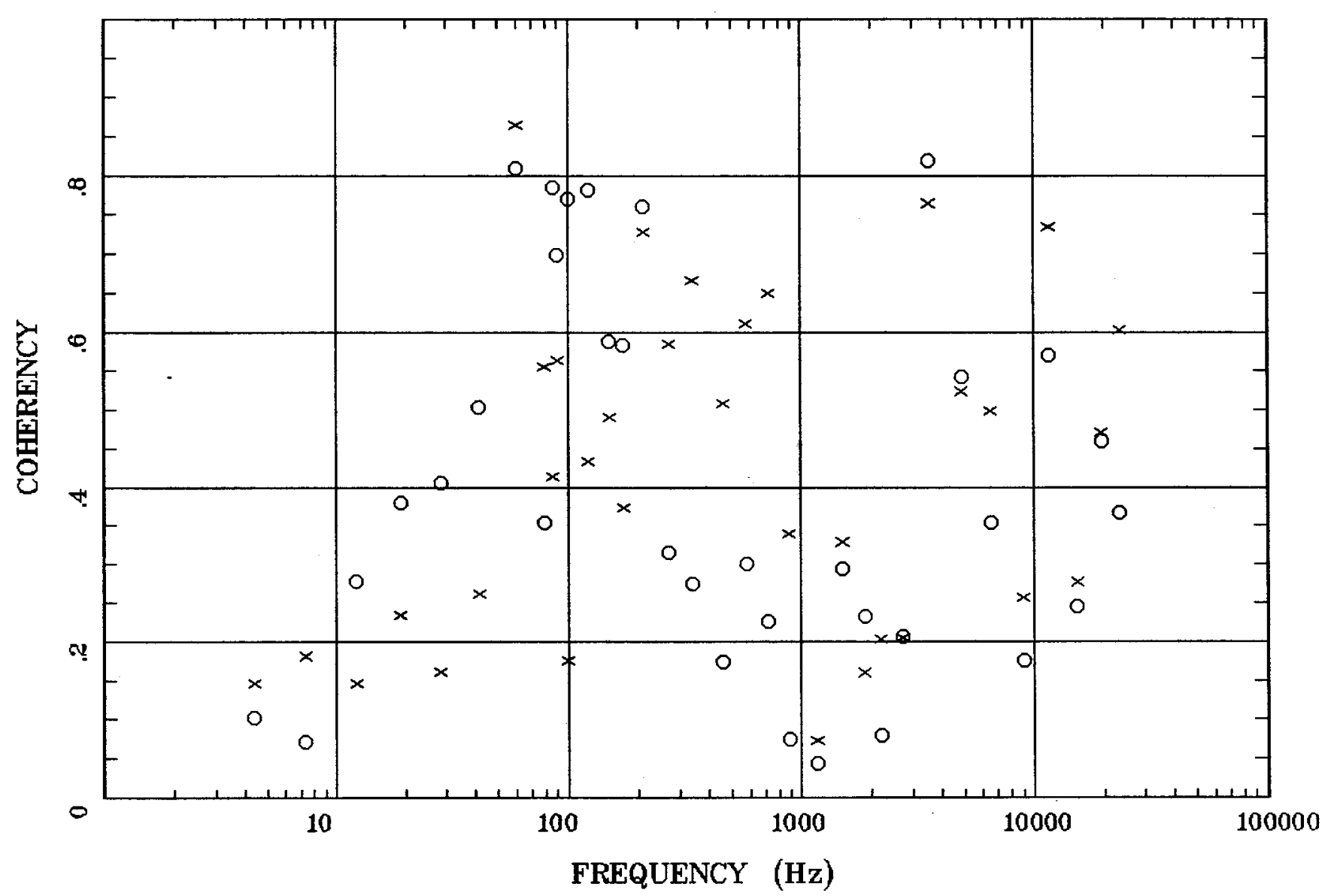

Client: Espanola Basin Remote: none

Acquired: 12:2 Jun 03, 2004 Survey co:USGS
Rotation:

Filename: cp 15aall.avg

Channels: Ch1 Ch2 Ch3 Ch4 Ch5 Ch3 Ch4

Plotted: 09:31 Aug 23, 2004

< EMI - ElectroMagnetic Instruments 


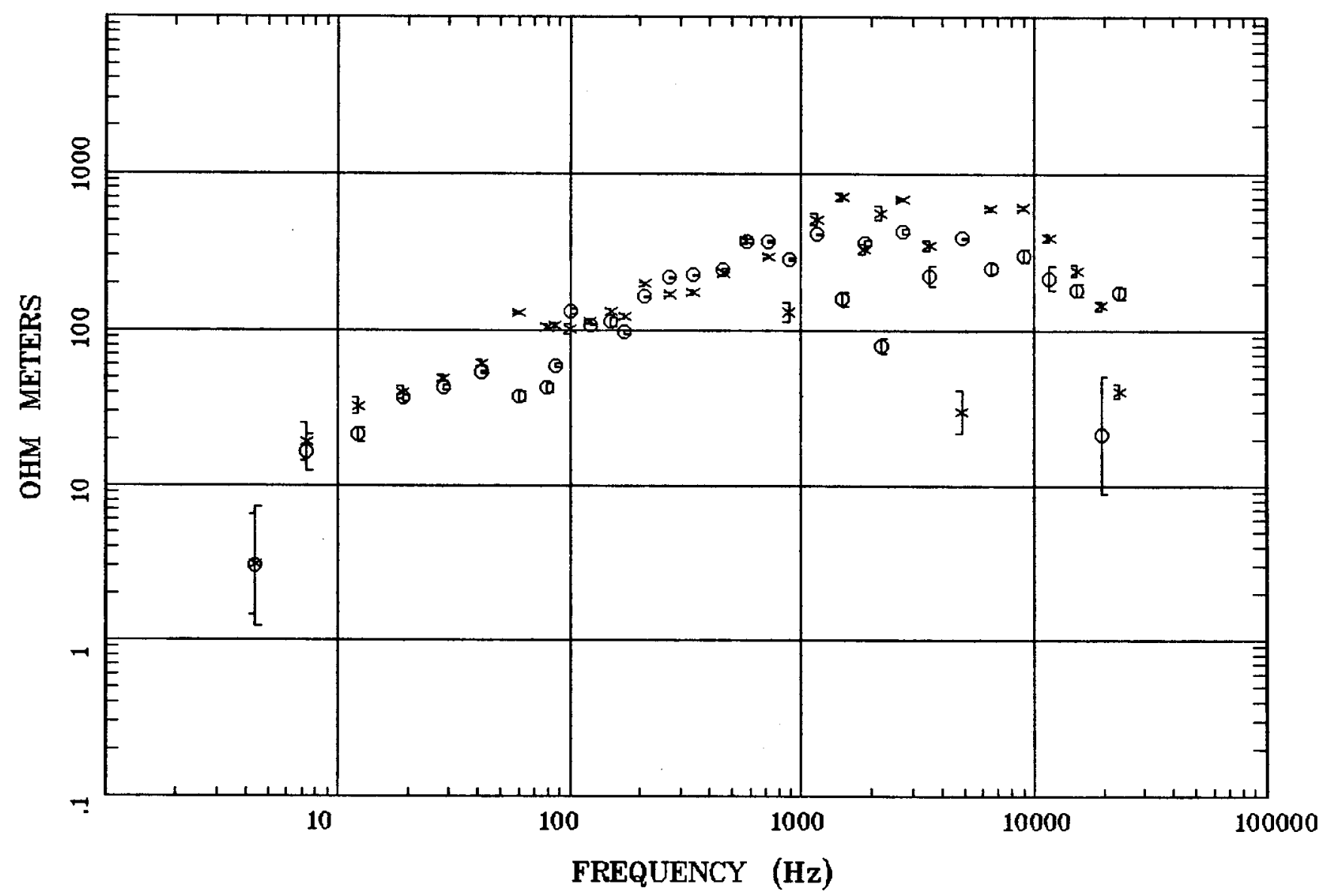

Client: Espanola Basin

Remote: none

Acquired: 16:1 Jun 03, 2004

Survey Co:USGS
Rotation:

Filename: cp14a.avg

Channels: Ch1 Ch2 Ch3 Ch4 Ch5 Ch3 Ch4

Plotted: 09:32 Aug 23, 2004

< EMI - ElectroMagnetic Instruments > 


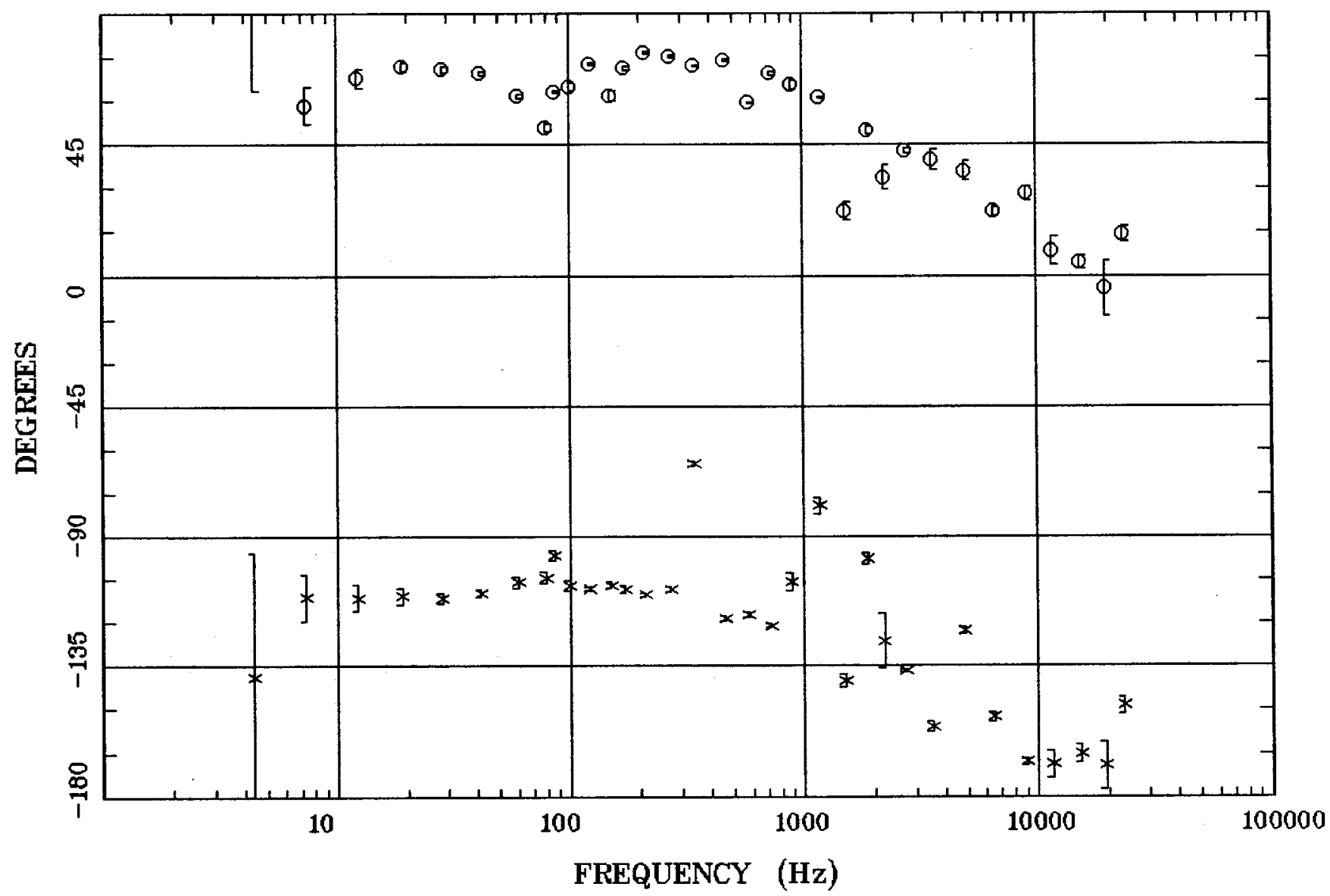

Client: Espanola Basin

Remote: none

Acquired: 16:1 Jun 03, 2004 Survey Co:USGS
Rotation:

Filename: cp14a.avg

Channels: Ch1 Ch2 Ch3 Ch4 Ch5 Ch3 Ch4 Plotted: 09:32 Aug 23, 2004

< EMI - ElectroMagnetic Instruments > 


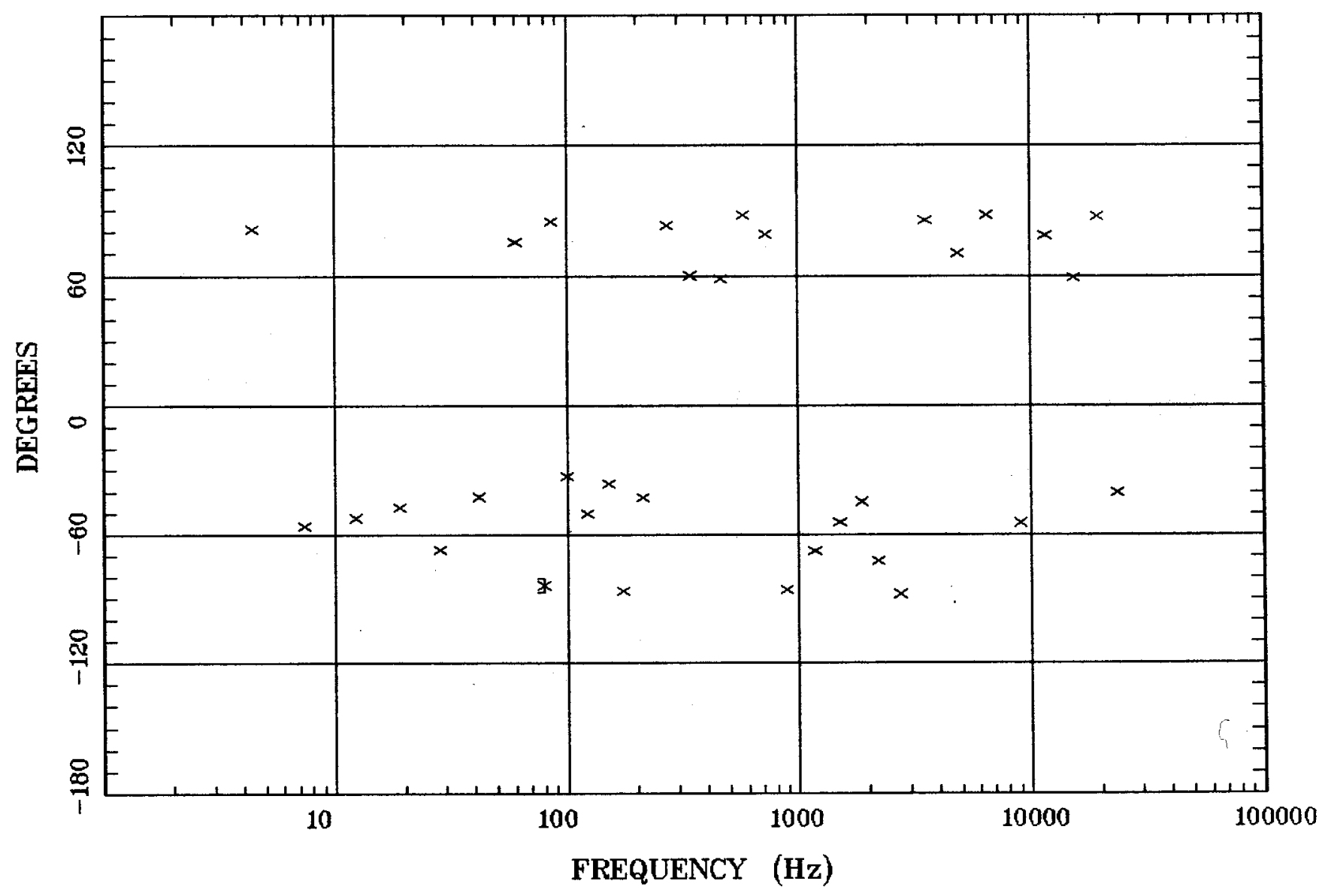

Client: Espanola Basin Remote: none Acquired: 16:1 Jun 03, 2004 Survey Co:USGS
Rotation:

Filename: cp 14a.avg

Channels: Ch1 Ch2 Ch3 Ch4 Ch5 Ch3 Ch4 Plotted: 09:32 Aug 23, 2004

< EMI - ElectroMagnetic Instruments 


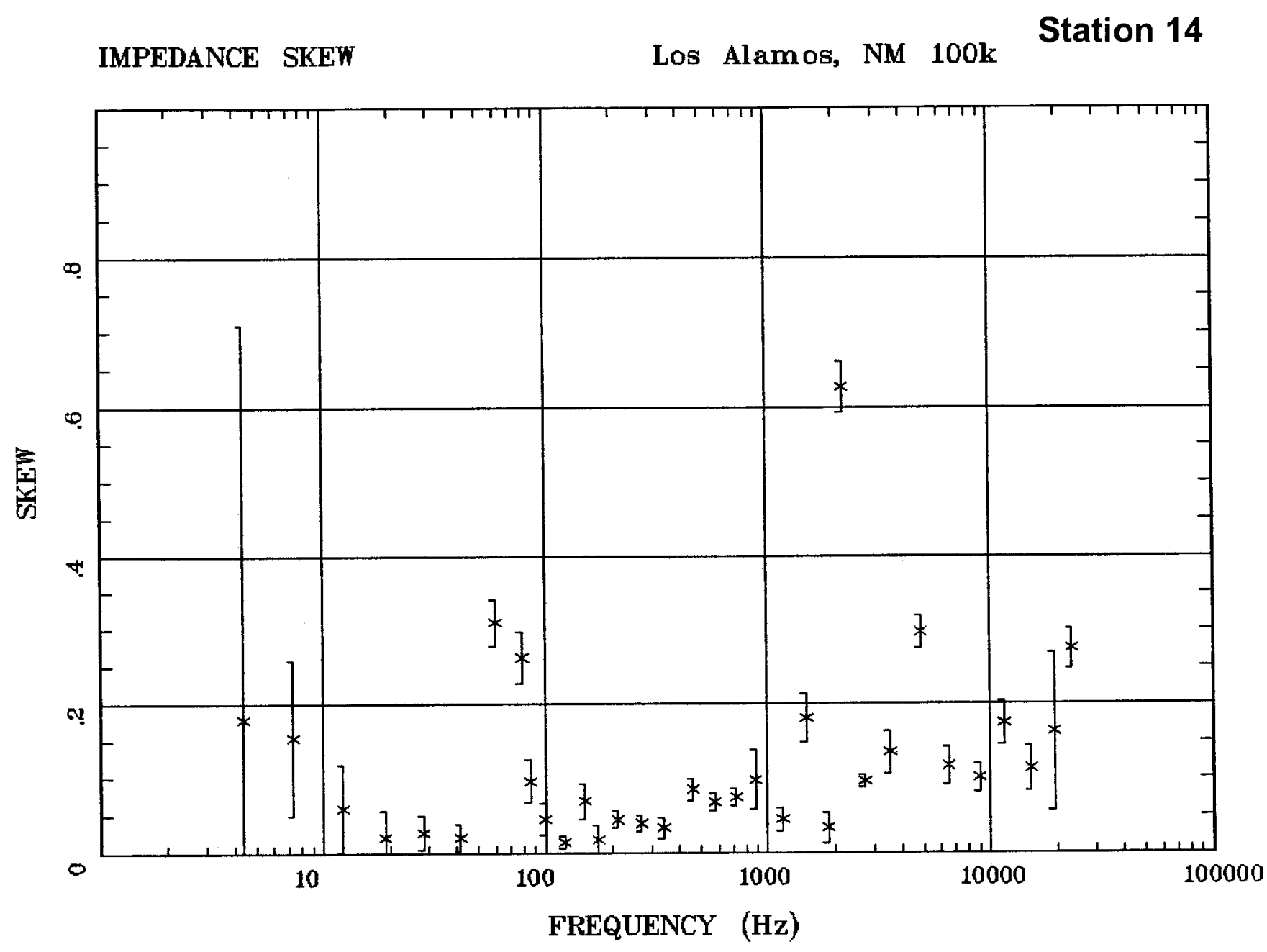

Client: Espanola Basin

Remote: none

Acquired: 16:1 Jun 03, 2004 Survey Co:USGS
Rotation:

Filename: cp 14a.avg

Channels: Ch1 Ch2 Ch3 Ch4 Ch5 Ch3 Ch4 Plotted: 09:32 Aug 23, 2004

< EMI - ElectroMagnetic Instruments 


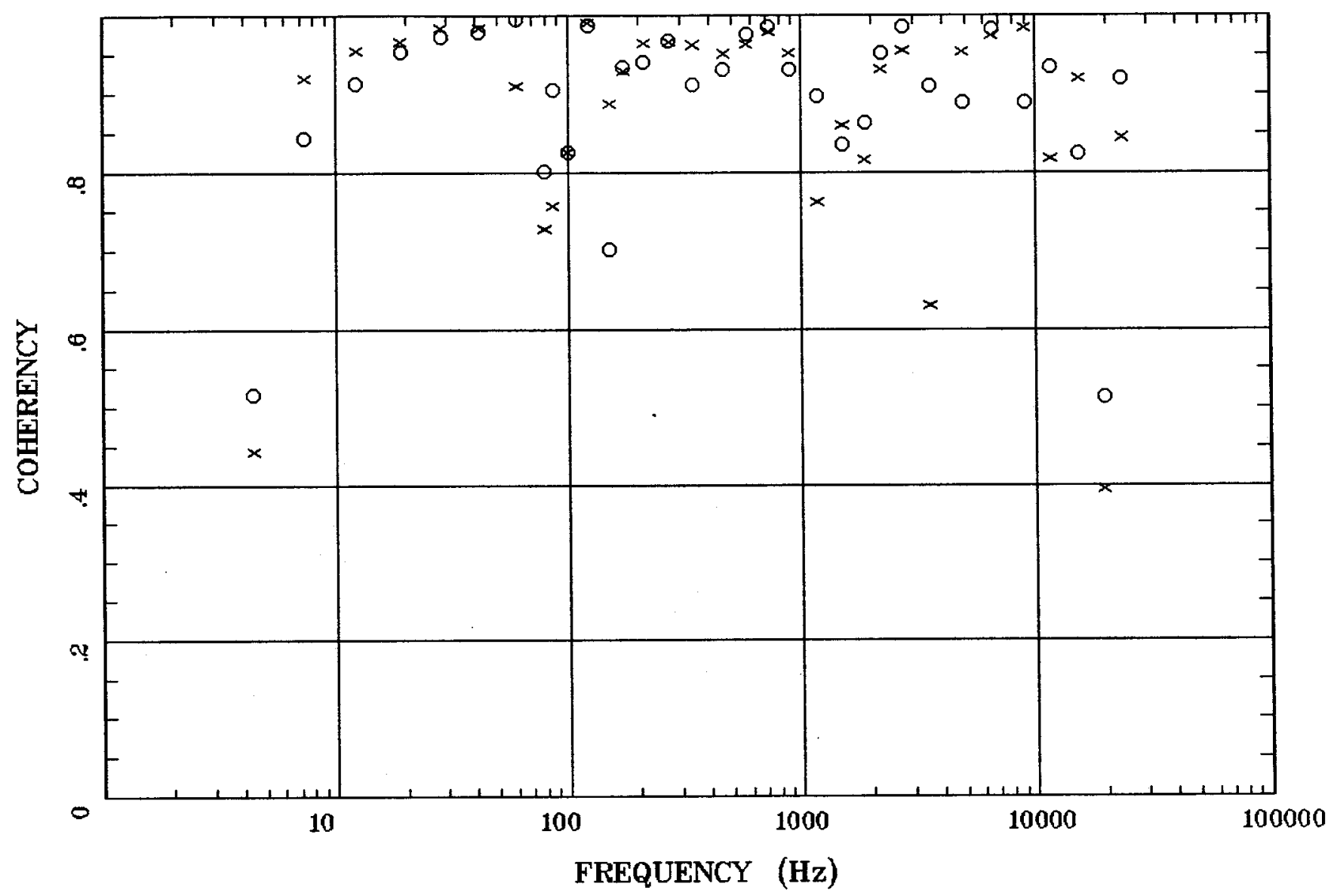

Client: Espanola Basin

Remote: none

Acquired: 16:1 Jun 03, 2004

Survey Co:USGS
Rotation:

Filename: cp 14a.avg

Channels: Ch1 Ch2 Ch3 Ch4 Ch5 Ch3 Ch4

Plotted: 09:32 Aug 23, 2004

< EMI - ElectroMagnetic Instruments > 


\section{POLAR PLOTS}

\section{Los Alamos, NM 100k}

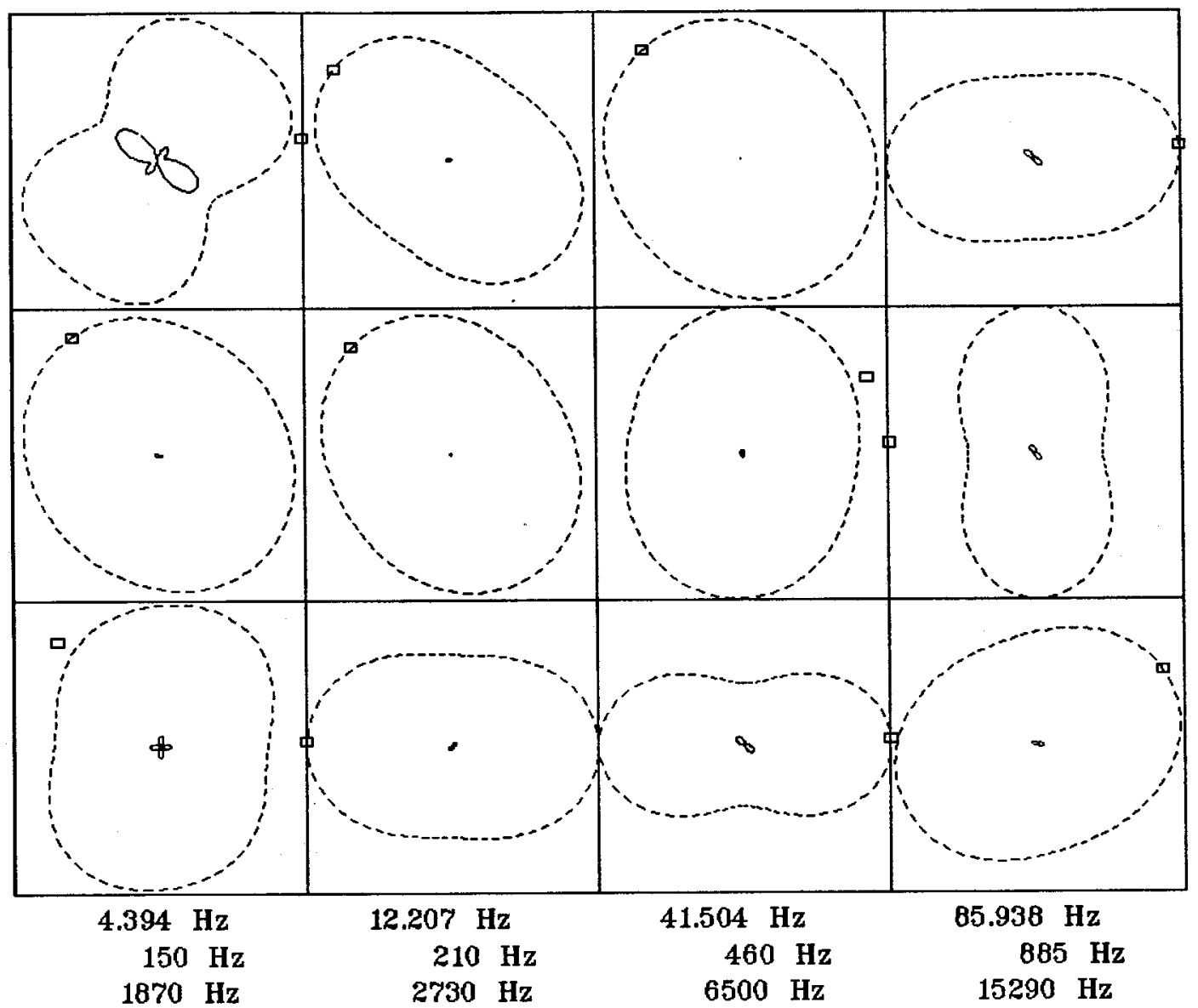

Client: Espanola Basin

Remote: none

Acquired: 16:1 Jun 03, 2004 Survey Co:USGS
Rotation:

Filename: cp 14a.evg

Channels: Ch1 Ch2 Ch3 Ch4 Ch5 Ch3 Ch4

Plotted: 09:32 Aug 23, 2004

$<$ EMI - ElectroMagnetic Instruments > 


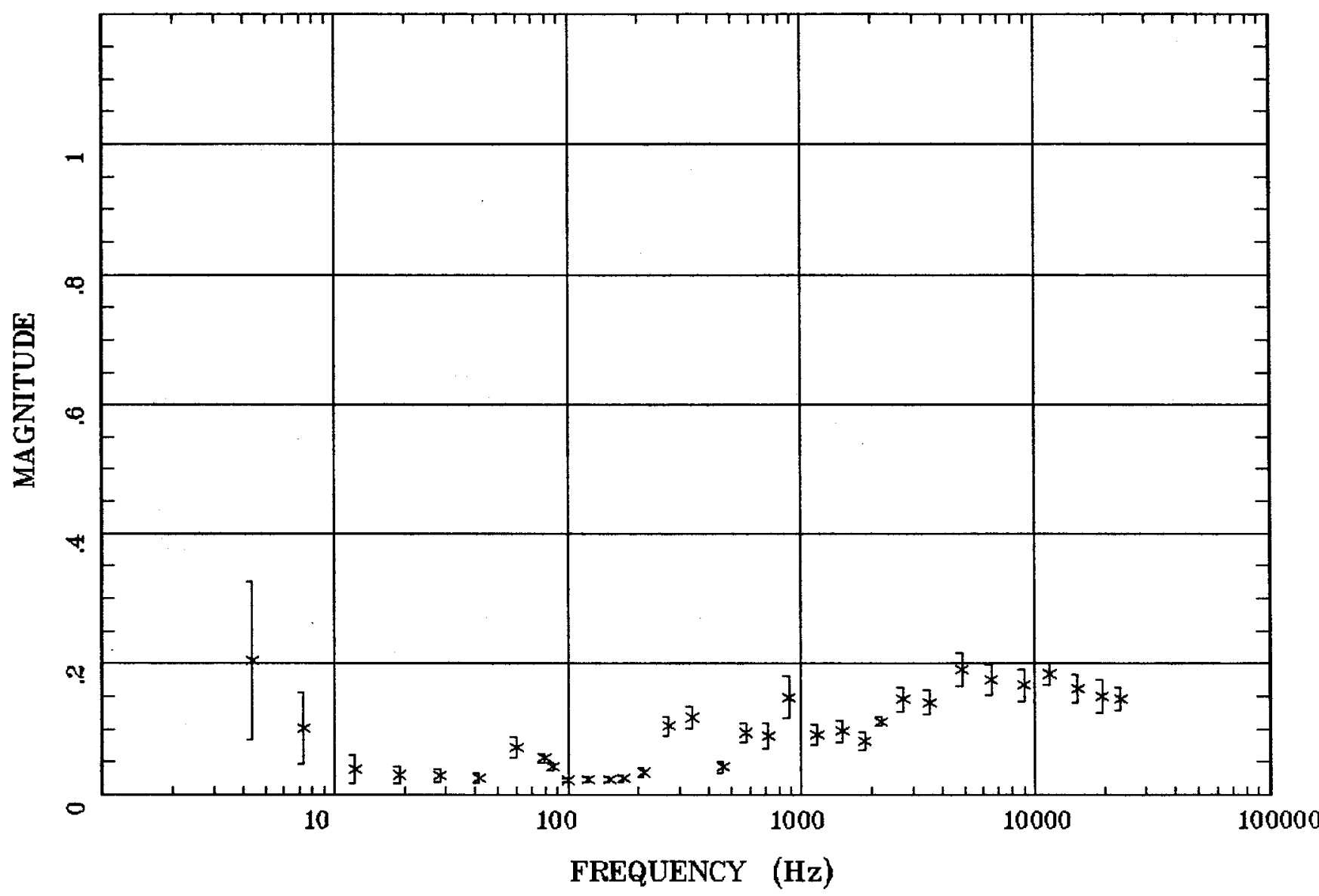

Client: Espanola Basin Remote: none Acquired: 16:1 Jun 03, 2004 Survey Co:USGS

\section{Rotation:}

Filename: cp14a.avg Channels: Ch1 Ch2 Ch3 Ch4 Ch5 Ch3 Ch4 Plotted: 09:32 Aug 23, 2004

< EMI - ElectroMagnetic Instruments 


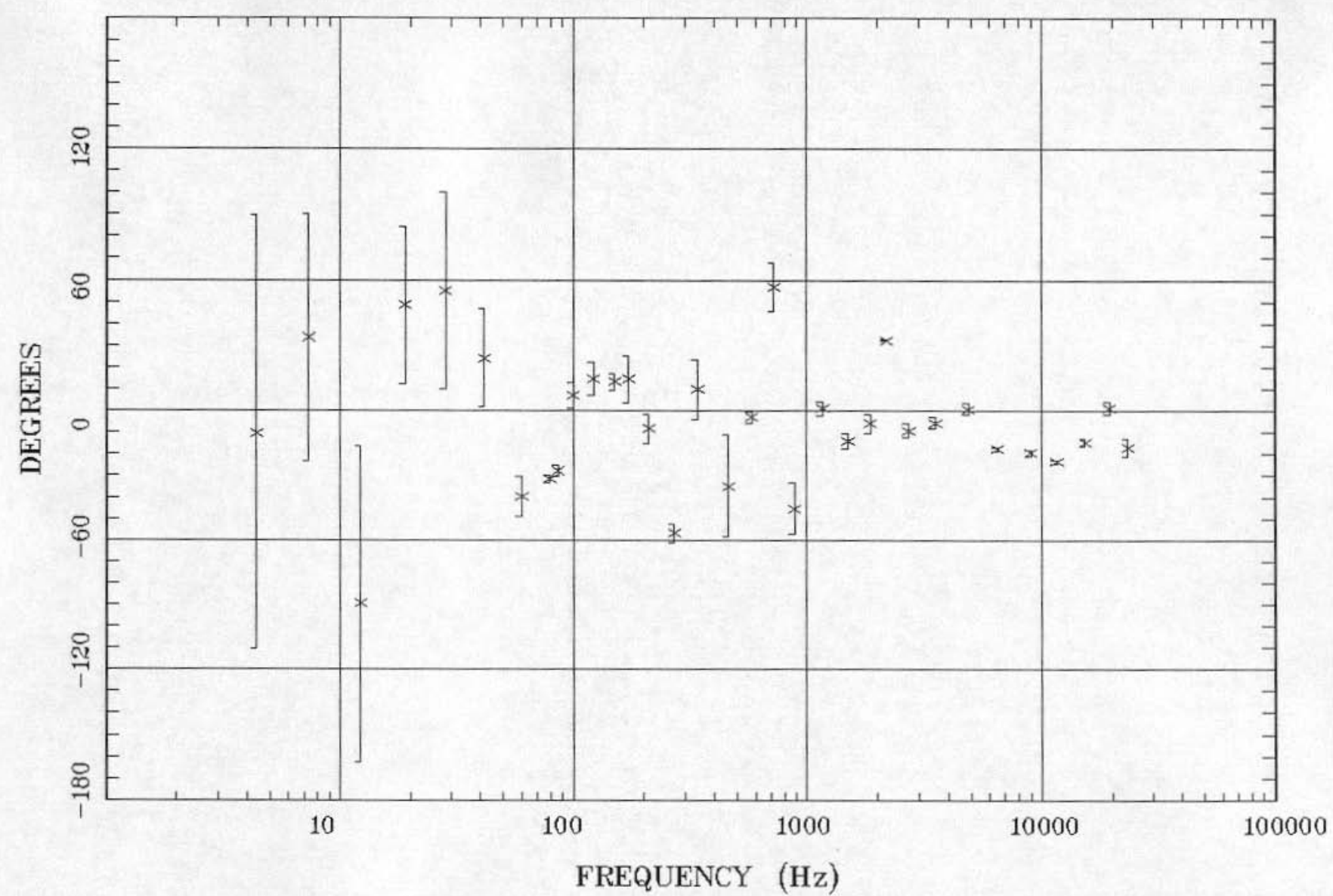

Client: Espanola Basin

Remote: none

Acquired: 16:1 Jun 03, 2004

Survey Co:USGS
Rotation:

Filename: cp 14a.avg

Channels: Ch1 Ch2 Ch3 Ch4 Ch5 Ch3 Ch4

Plotted: 09:32 Aug 23, 2004

< EMI - ElectroMagnetic Instruments > 
Los Alamos, NM 100k

Station 14

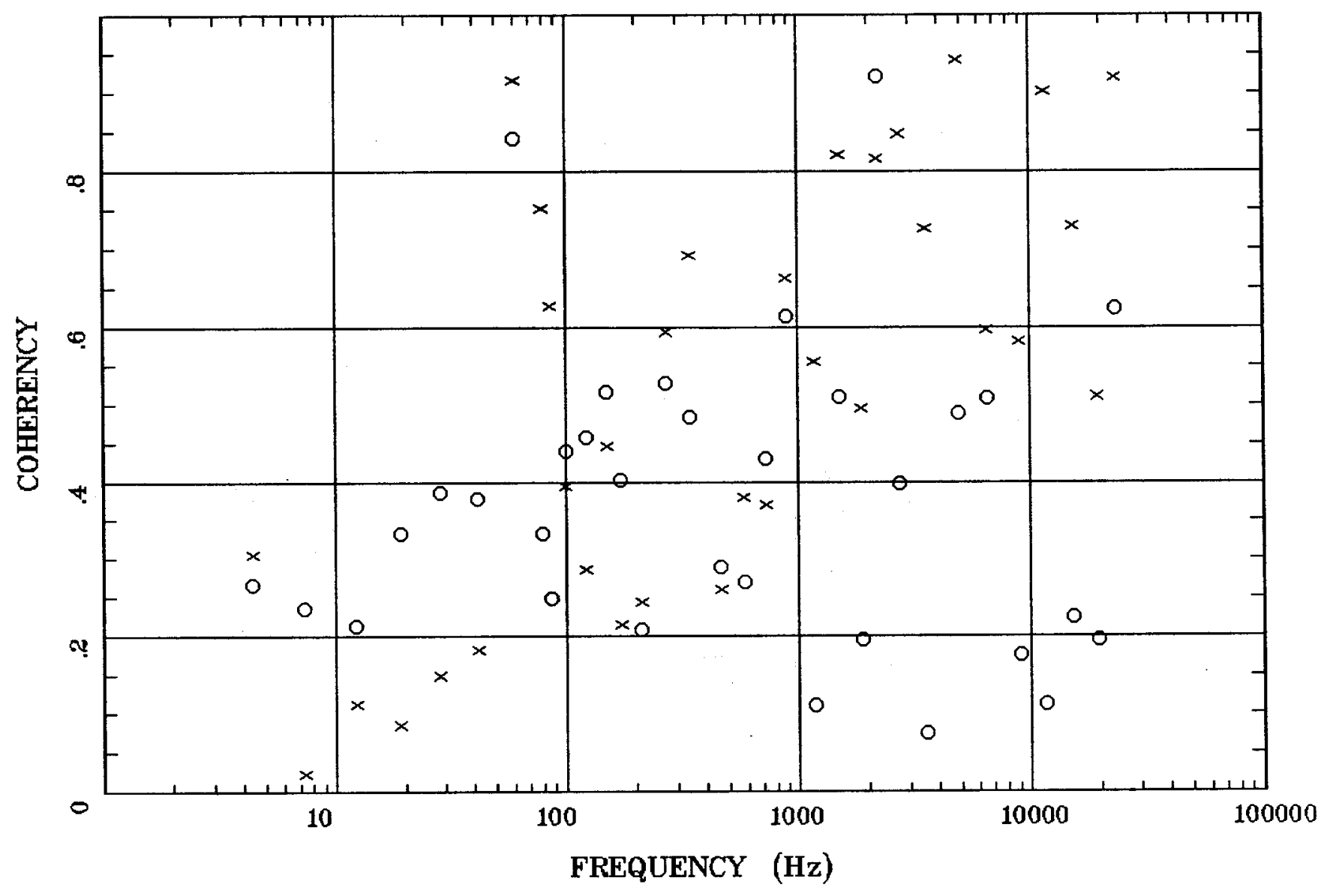

Client: Espanola Basin

Remote: none

Acquired: 16:1 Jun 03, 2004 Survey Co:USGS
Rotation:

Filename: cp 14a.avg

Channels: Ch1 Ch2 Ch3 Ch4 Ch5 Ch3 Ch4

Plotted: 09:32 Aug 23, 2004

< EMI - ElectroMagnetic Instruments > 


\section{Station 29}

APPARENT RESISTIVITY

Los Alamos, NM 100k

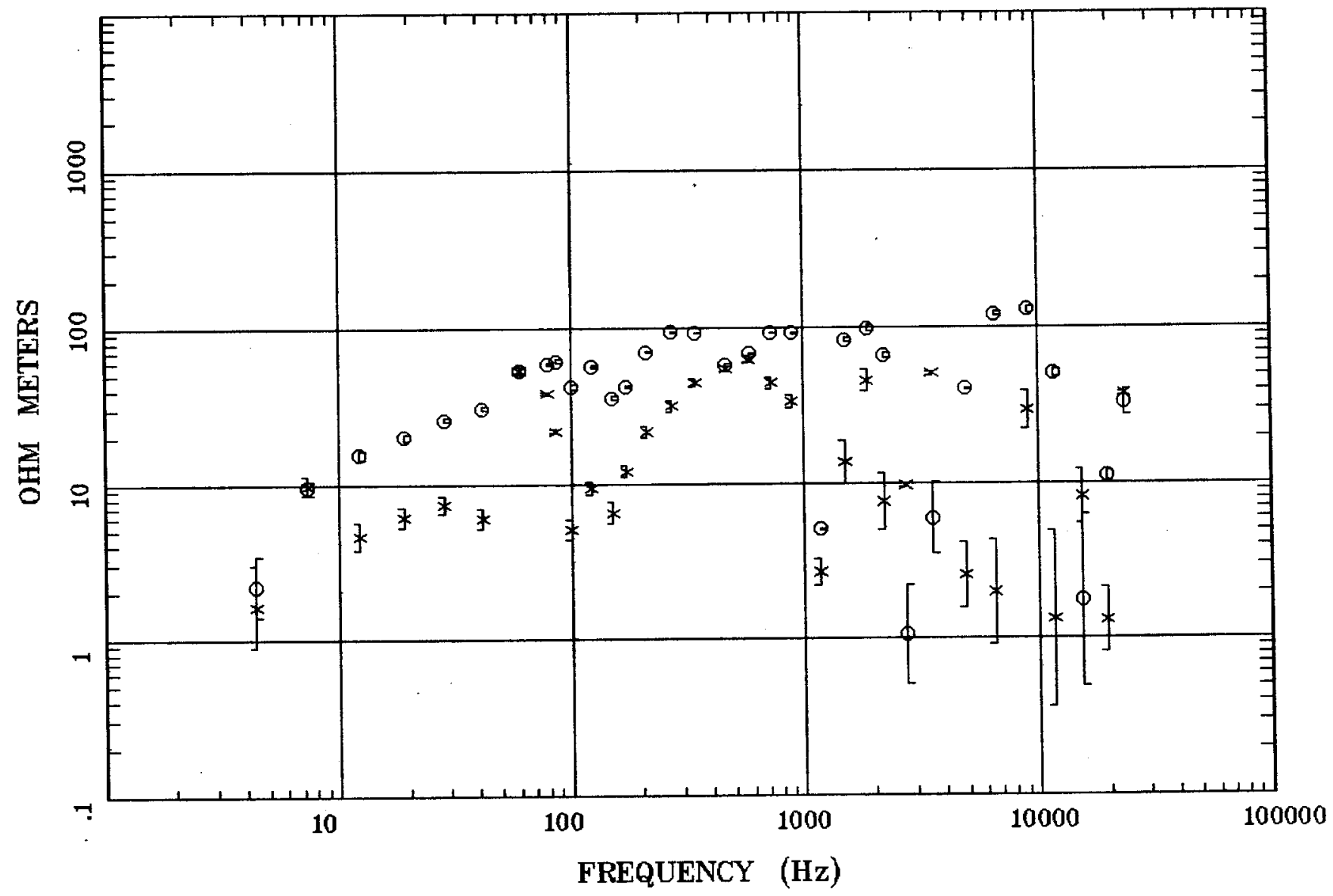

Client: Espanola Basin

Remote: none

Acquired: 13:4 Jun 06, 2004

Survey Co:USGS
Rotation:

Filename: cp29b.avg

Channels: Ch1 Ch2 Ch3 Ch4 Ch5 Ch3 Ch4

Plotted: 09:18 Aug 23, 2004

< EMI - ElectroMagnetic Instruments > 
IMPEDANCE PHASE

Los Alamos, NM 100k

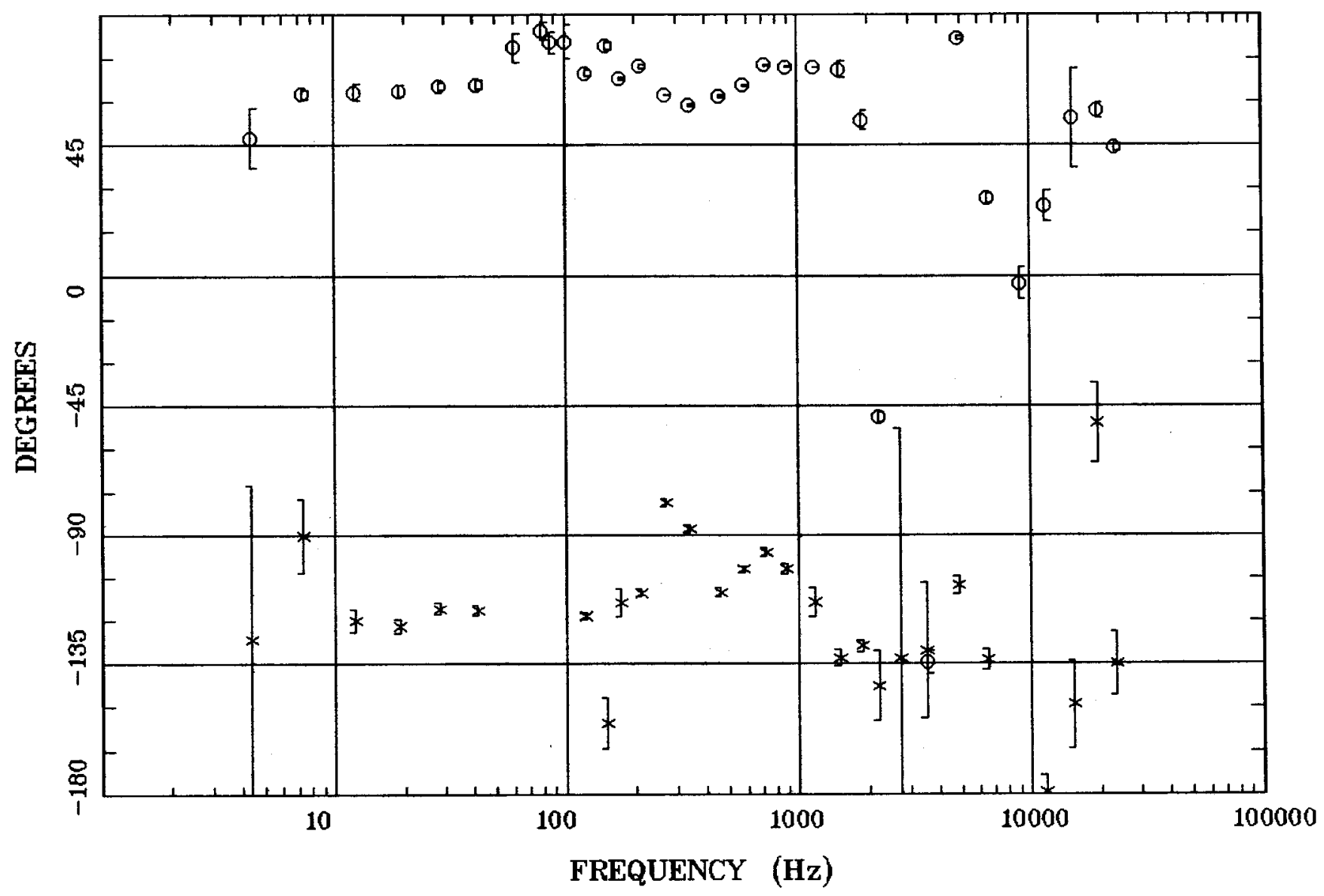

Client: Espanola Basin

Remote: none

Acquired: 13:4 Jun 06, 2004 Survey Co:USGS
Rotation:

Filename: cp29b.avg

Channels: Ch1 Ch2 Ch3 Ch4 Ch5 Ch3 Ch4 Plotted: 09:18 Aug 23, 2004

< EMI - ElectroMagnetic Instruments 
Station 29

ROTATION ANGLE

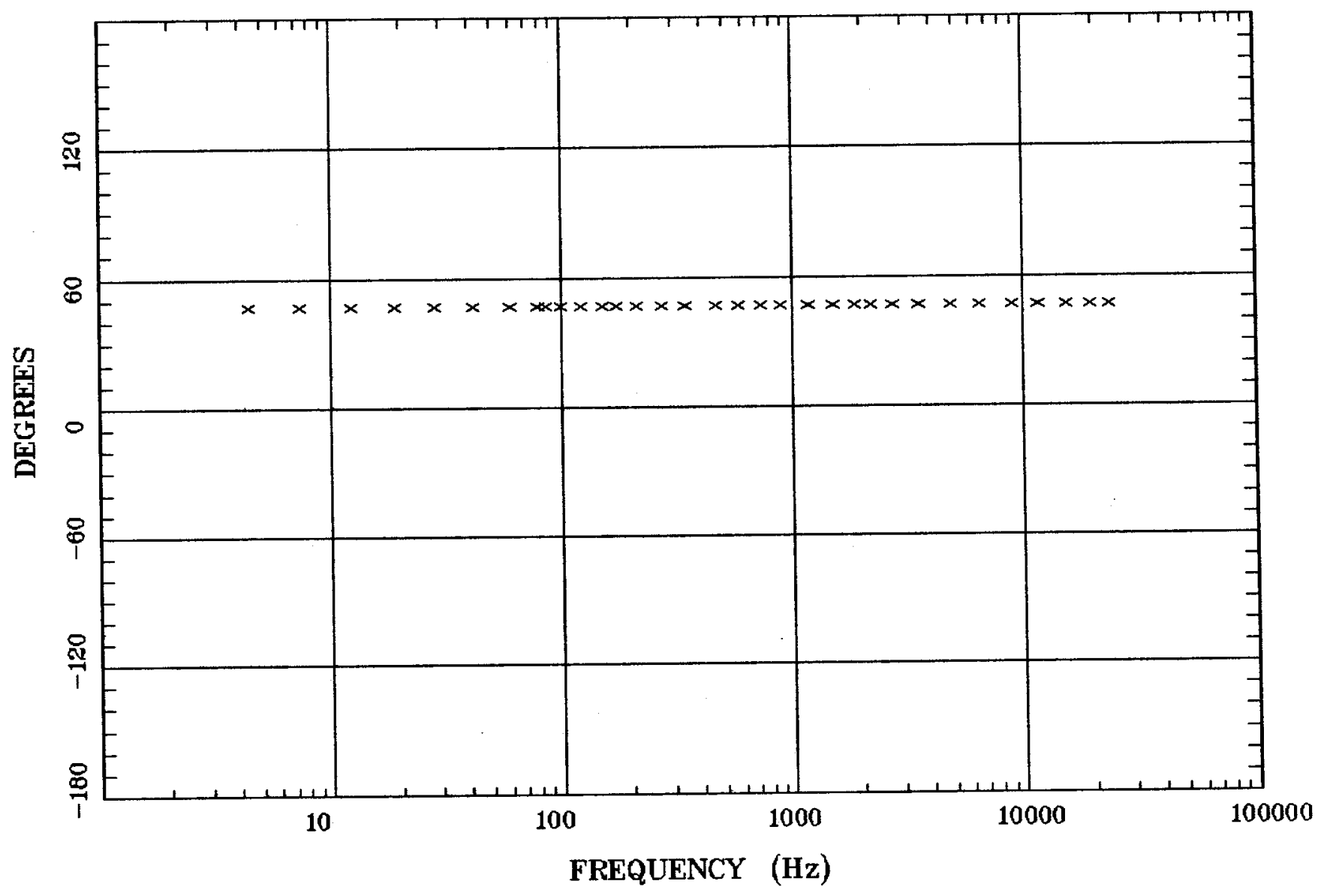

Client: Espanola Basin

Remote: none

Acquired: 13:4 Jun 06, 2004

Survey Co:USGS
Rotation:

Los Alamos, NM 100k

Filename: cp29b.avg

Plotted: 09:18 Aug 23, 2004

< EMI - ElectroMagnetic Instruments
Channels: Ch1 Ch2 Ch3 Ch4 Ch5 Ch3 Ch4 


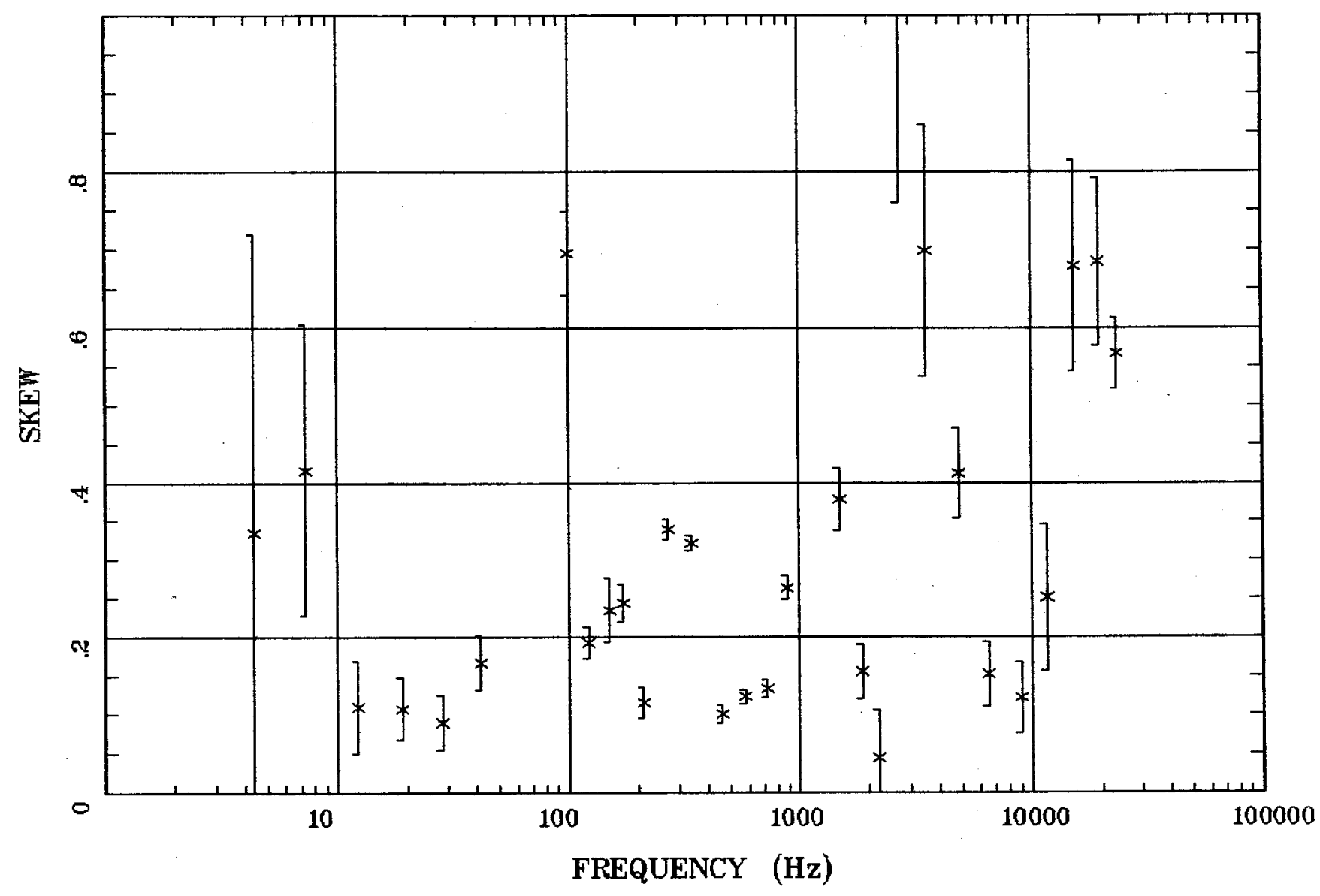

Client: Espanola Basin Remote: none

Acquired: 13:4 Jun 06, 2004 Survey Co:USGS
Rotation:

Filename: cp29b.avg

Channels: Ch1 Ch2 Ch3 ch4 Ch5 Ch3 Ch4 Plotted: 09:18 Aug 23, 2004

< EMI - ElectroMagnetic Instruments 
E MULT Coh.

Los Alamos, NM 100k

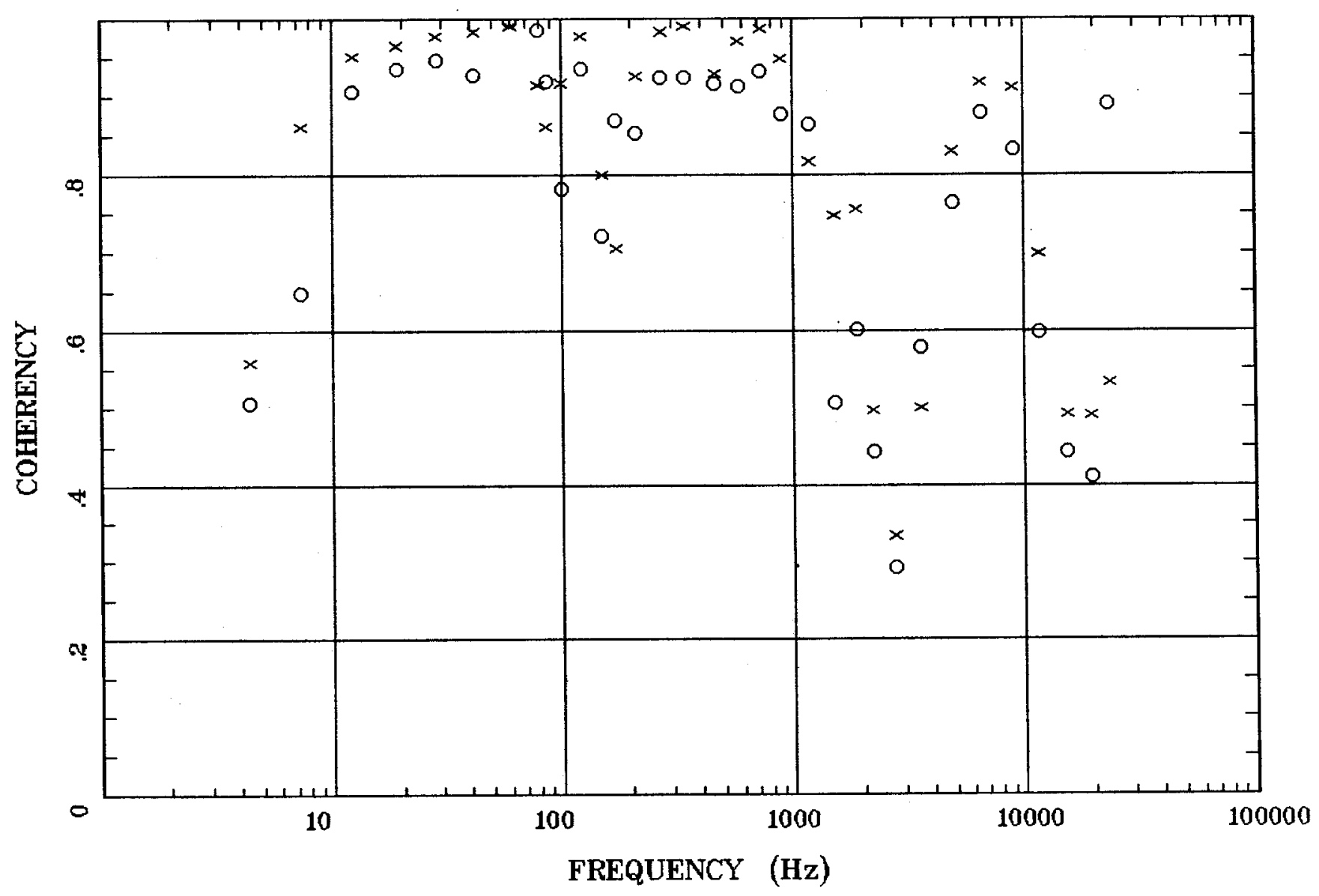

Client: Espanola Basin

Remote: none

Acquired: 13:4 Jun 06, 2004 Survey Co:USGS
Rotation:

Filename: cp 29b.avg

Channels: Ch1 Ch2 Ch3 Ch4 Ch5 Ch3 Ch4 Plotted: 09:18 Aug 23, 2004

$<$ EMI - ElectroMagnetic Instruments 


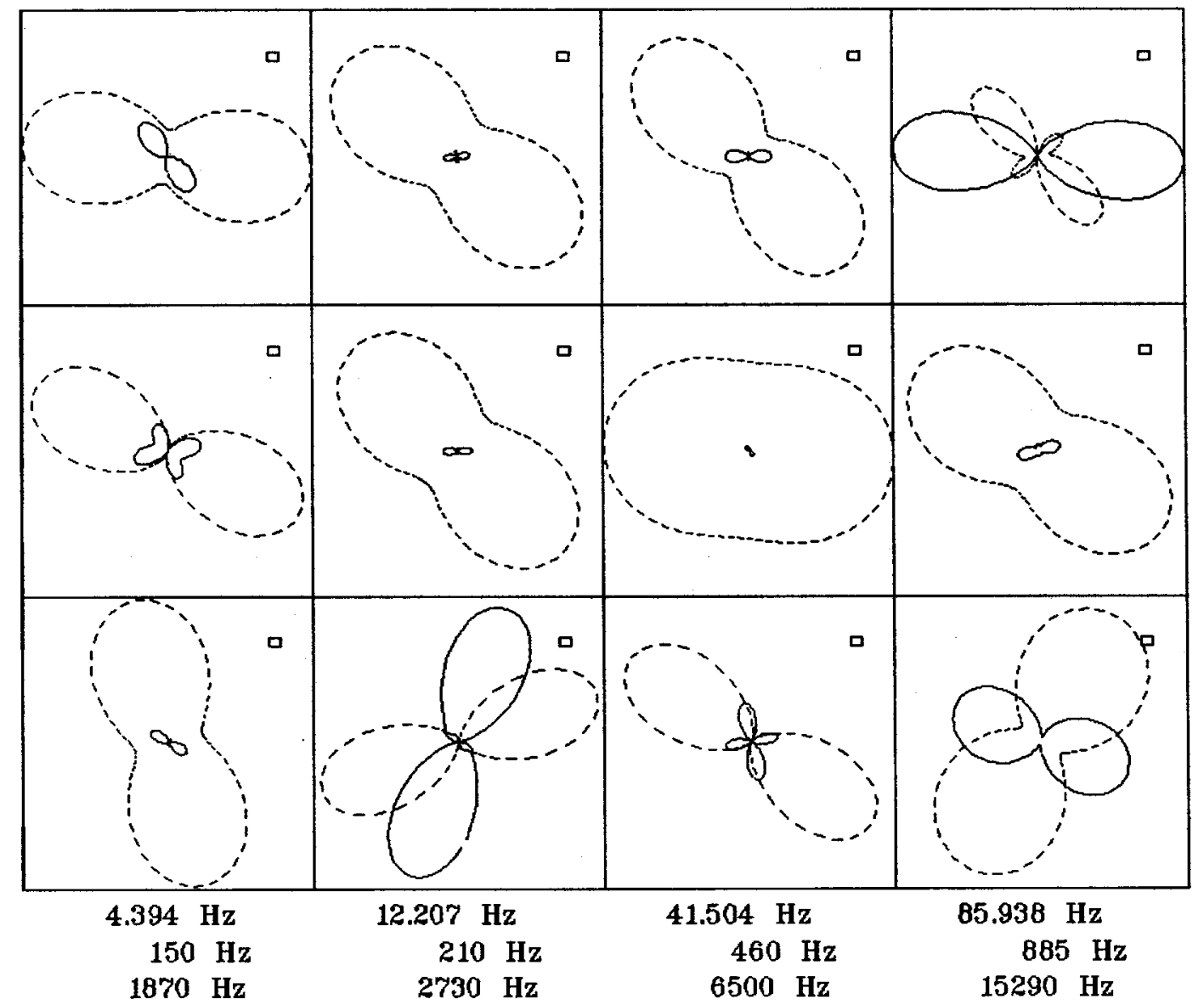

Client: Espanola Basin

Remote: none

Acquired: 13:4 Jun 06, 2004 Survey Co:USGS
Rotation:

Filename: cp29b.avg

Channels: Ch1 Ch2 Ch3 Ch4 Ch5 Ch3 Ch4 Plotted: 09:18 Aug 23, 2004

< EMI - ElectroMagnetic Instruments > 
Los Alamos, NM 100k

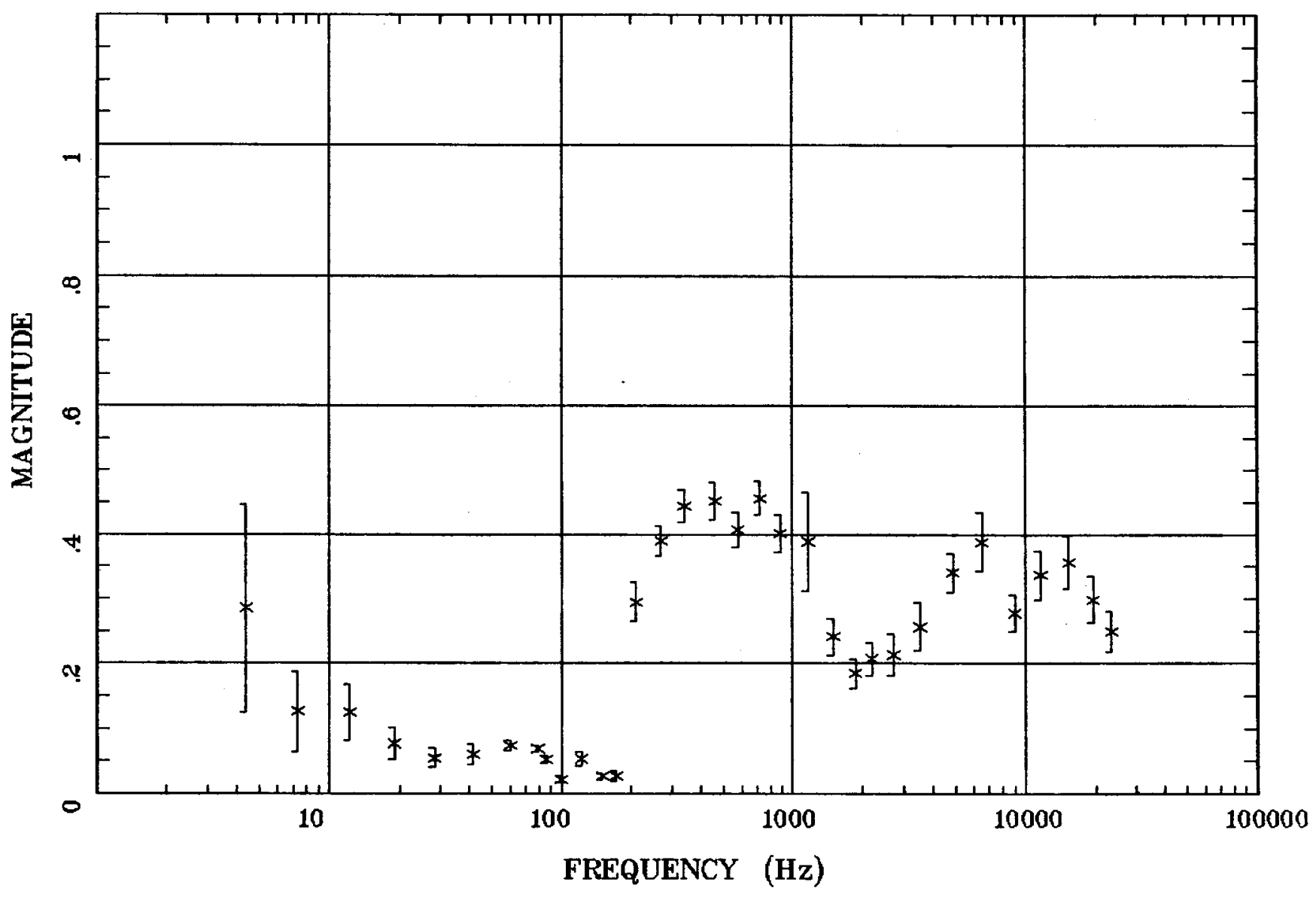

Client: Espanola Basin Remote: none Acquired: 13:4 Jun 06, 2004 Survey Co:USGS
Rotation:

Filename: cp29b.avg

Channels: Ch1 Ch2 Ch3 Ch4 Ch5 Ch3 Ch4 Plotted: 09:18 Aug 23, 2004

$<$ EMI - ElectroMagnetic Instruments 
TIPPER STRIKE

Los Alamos, NM $100 \mathrm{k}$

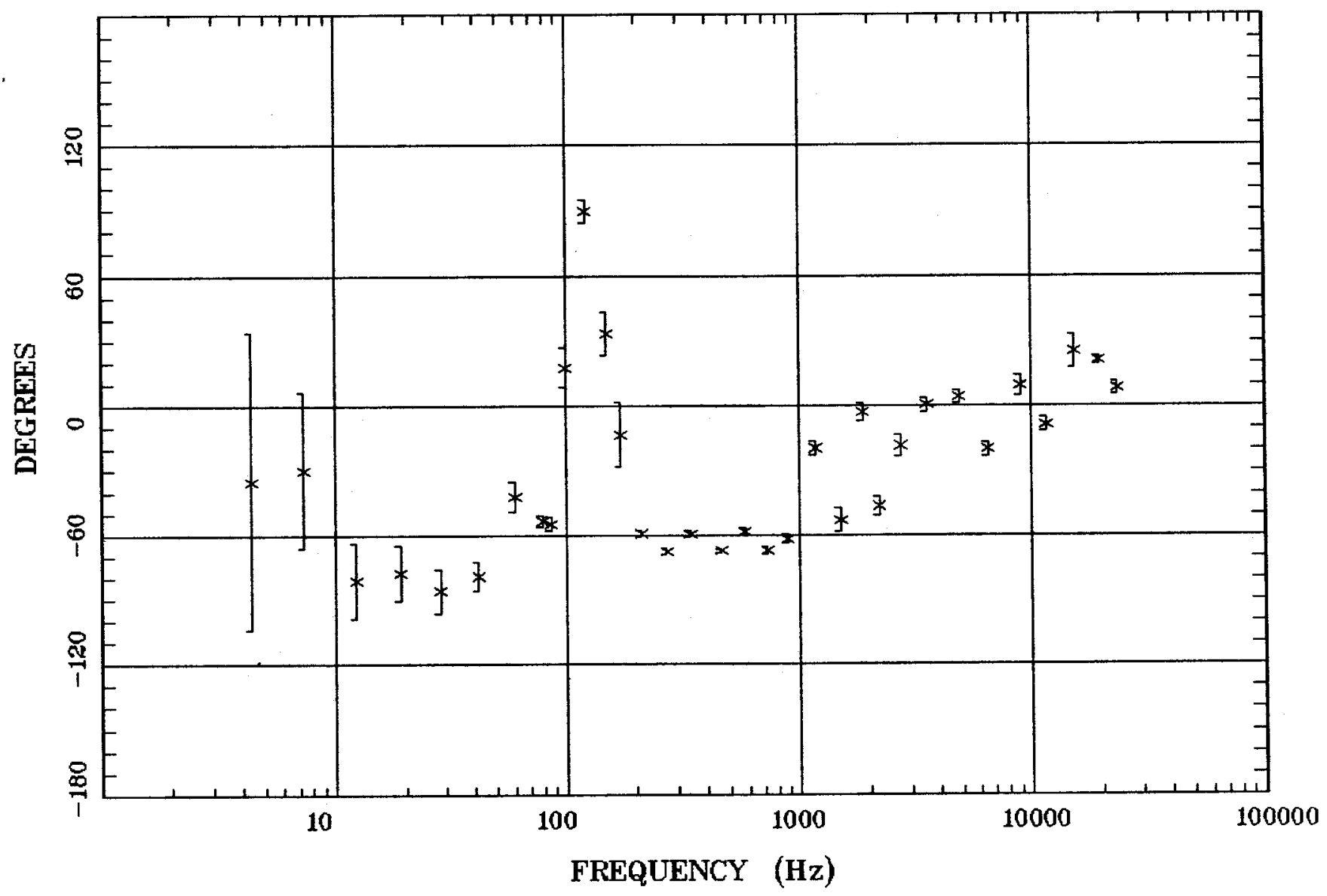

Client: Espanola Basin

Remote: none

Acquired: 13:4 Jun 06, 2004 Survey Co:USGS
Rotation:

Filename: cp29b.avg

Channels: Ch1 Ch2 Ch3 Ch4 Ch5 Ch3 Ch4 Plotted: 09:18 Aug 23, 2004

$<$ EMI - ElectroMagnetic Instruments > 
Station 29

HzHx.x Coh HzHy.o

Los Alamos, NM 100k

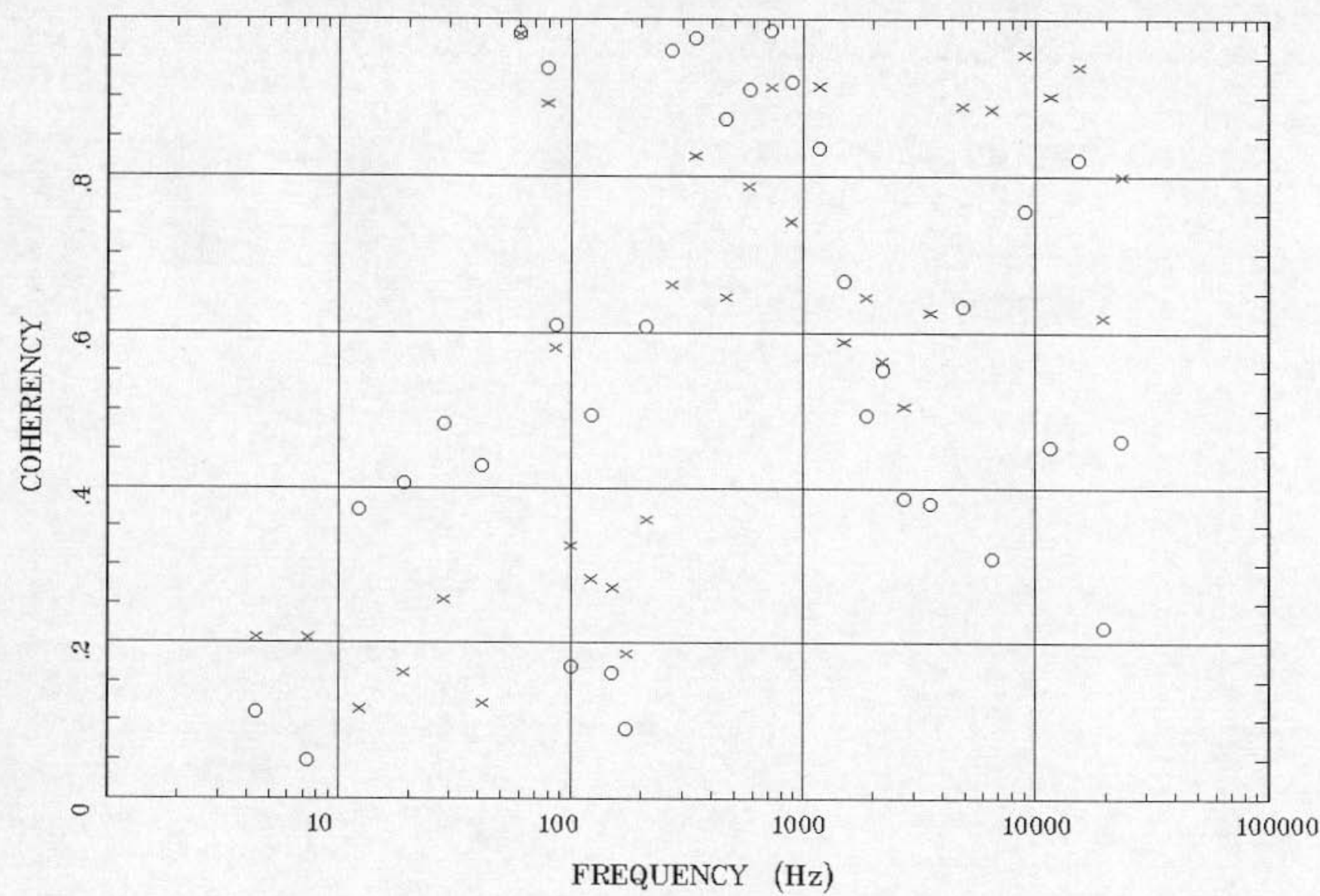

Client: Espanola Basin

Remote: none

Acquired: 13:4 Jun 06, 2004 Survey Co:USGS
Rotation:

Filename: cp29b.avg

Channels: Ch1 Ch2 Ch3 Ch4 Ch5 Ch3 Ch4

Plotted: 09:18 Aug 23, 2004

< EMI - ElectroMagnetic Instruments 


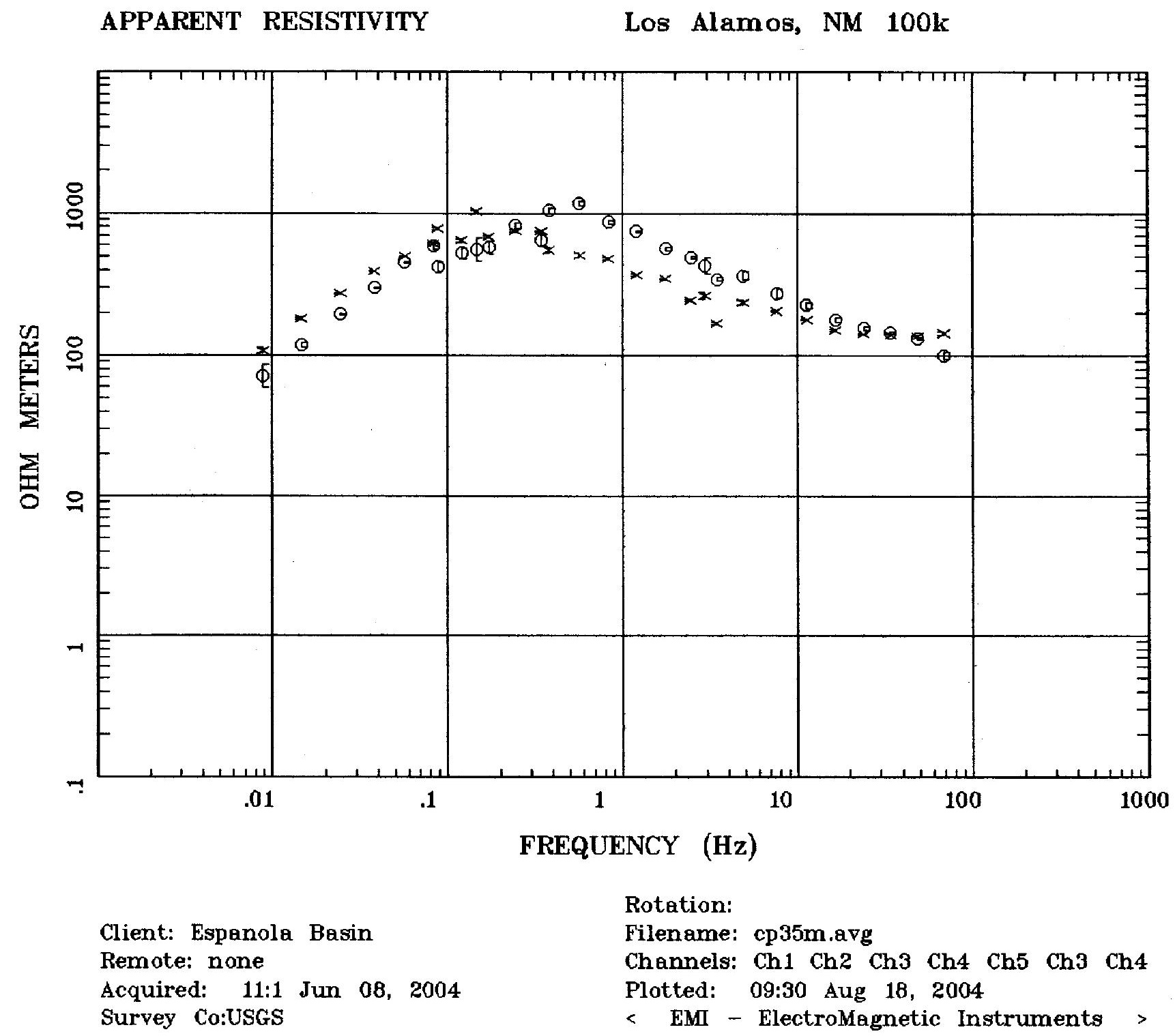


Station 35

IMPEDANCE PHASE

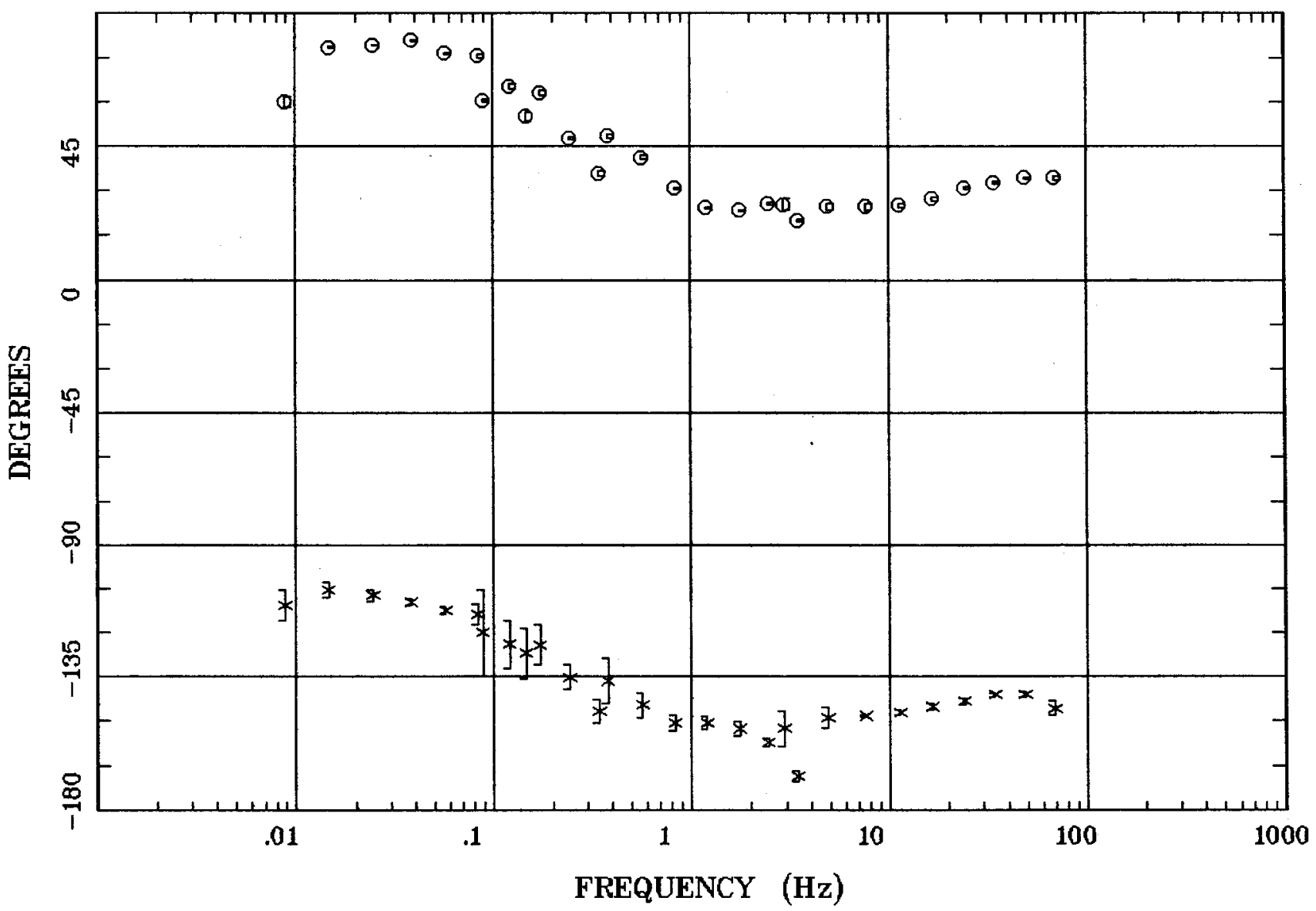

Client: Espanola Basin Remote: none

Acquired: 11:1 Jun 08, 2004 Survey Co:USGS
Los Alamos, NM 100k

Rotation:

Filename: cp35m.avg

Channels: Ch1 Ch2 Ch3 Ch4 Ch5 Ch3 Ch4

Plotted: 09:30 Aug 18, 2004

< EMI - ElectroMagnetic Instruments > 


\section{Station 35}

IMPEDANCE SKEW

Los Alamos, NM 100k

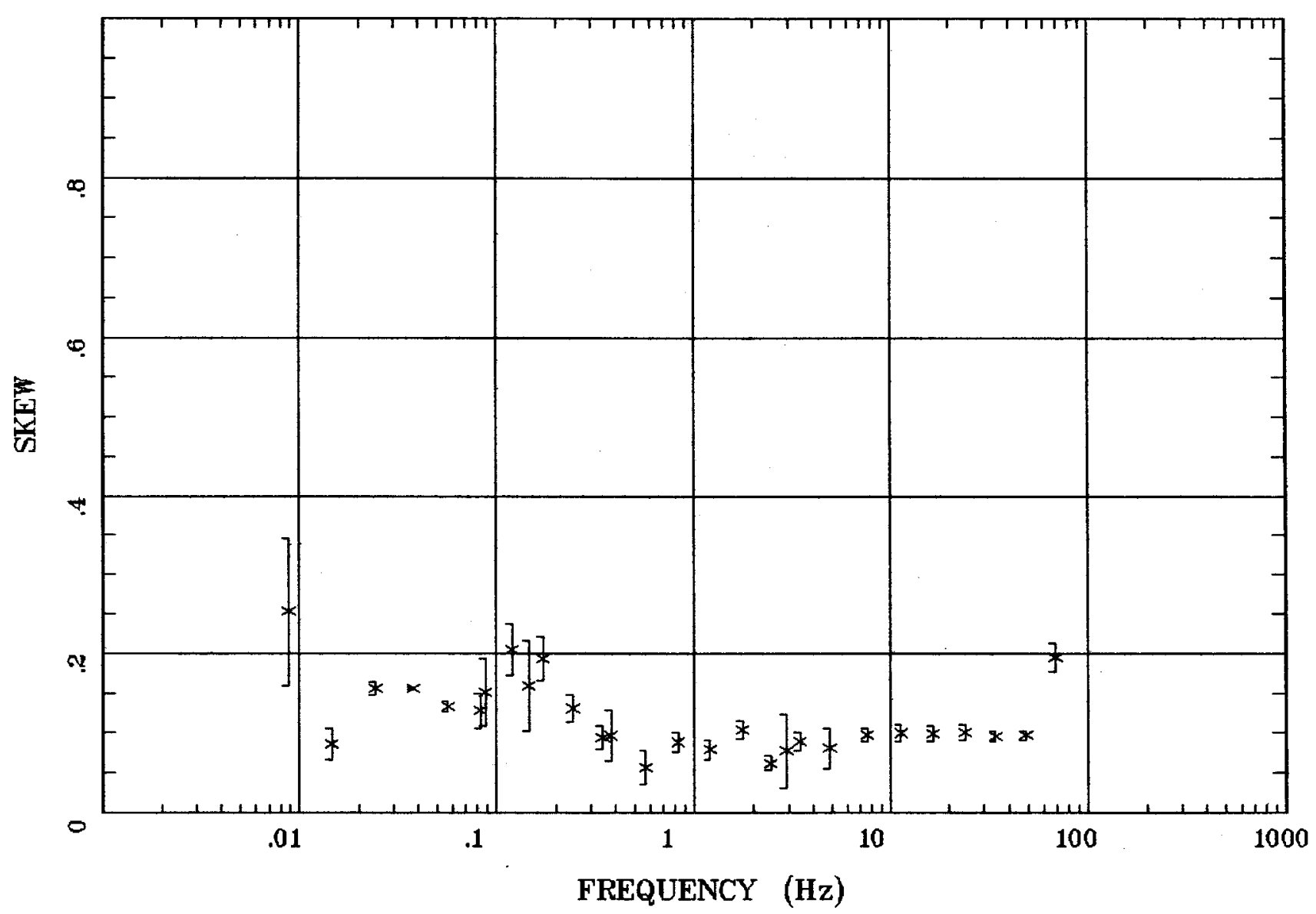

Client: Espanola Basin

Remote: none

Acquired: 11:1 Jun 08, 2004 Survey Co:USGS
Rotation:

Filename: cp35m.avg

Channels: Ch1 Ch2 Ch3 Ch4 Ch5 Ch3 Ch4 Plotted: 09:30 Aug 18, 2004

$<$ EMI - ElectroMagnetic Instruments 


\section{Station 35}

E MULT Coh.

Los Alamos, NM 100k

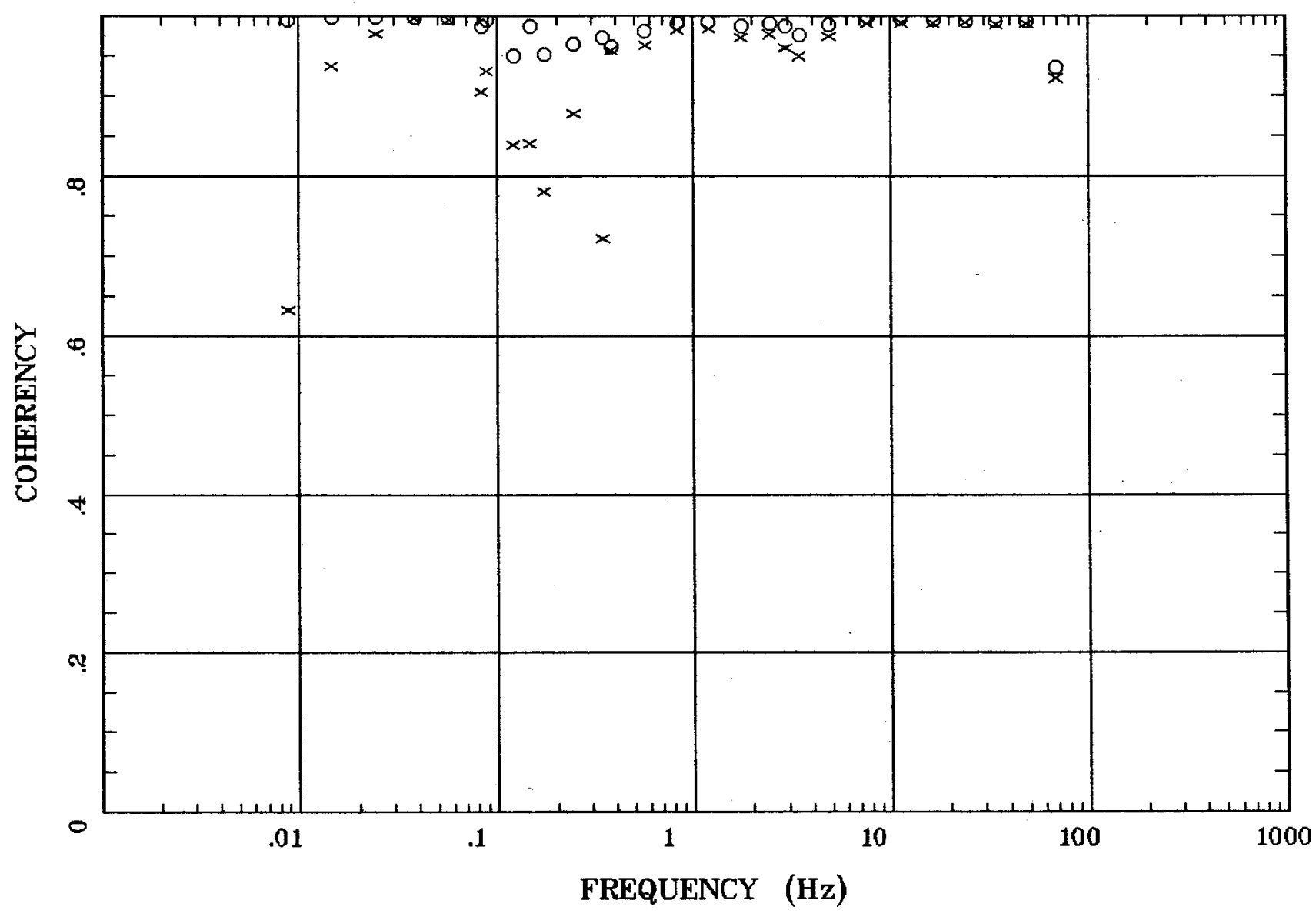

Client: Espanola Basin

Remote: none

Acquired: 11:1 Jun 08, 2004

Survey Co:USGS
Rotation:

Filename: cp35m.avg

Channels: Ch1 Ch2 Ch3 Ch4 Ch5 Ch3 Ch4 Platted: 09:30 Aug 18, 2004

$<$ EMI - ElectroMagnetic Instruments > 


\section{POLAR PLOTS}

Los Alamos, NM 100k

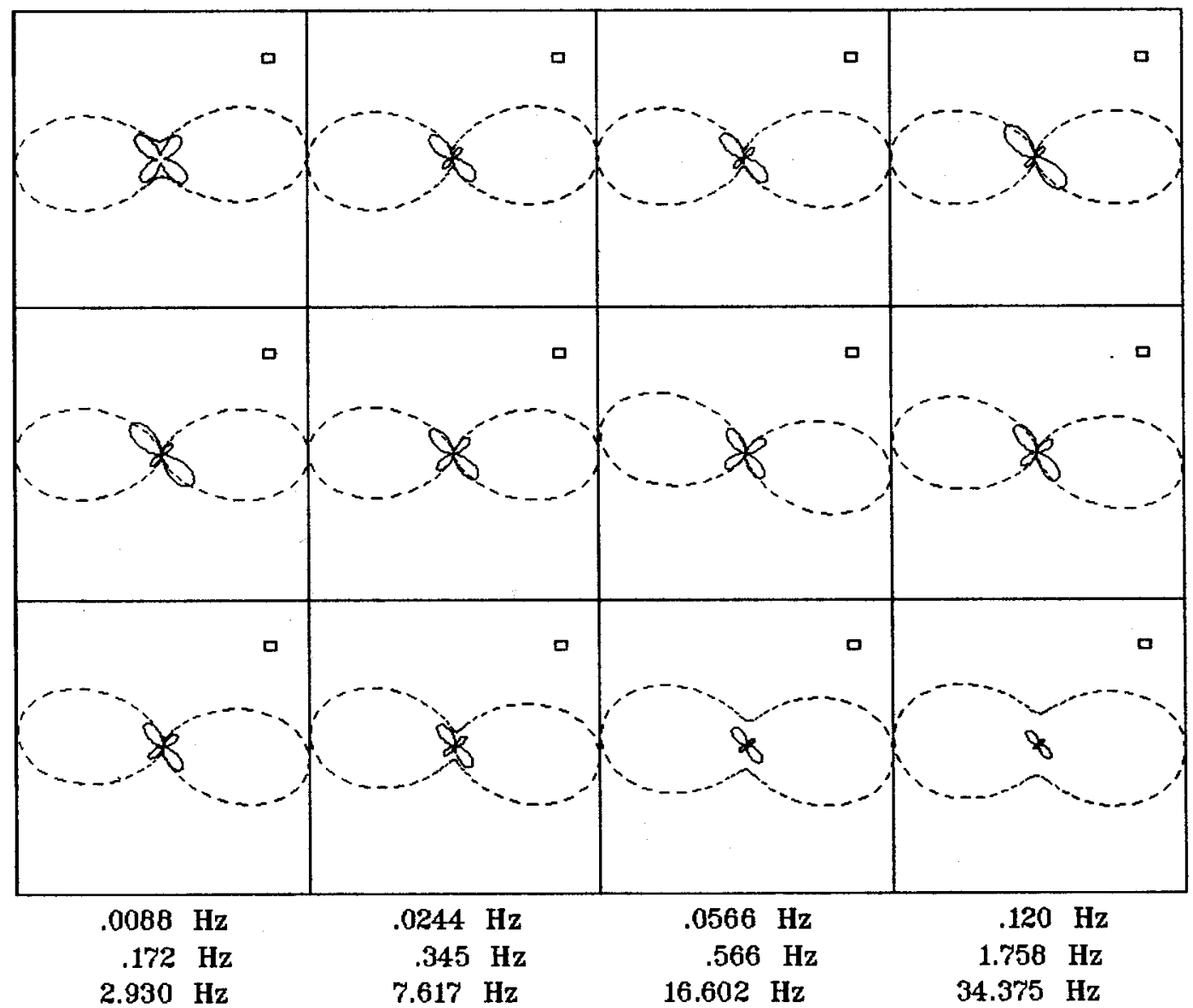

Client: Espanola Basin

Remote: none

Acquired: 11:1 Jun 08, 2004

Survey Co:USGS
Rotation:

Filename: cp35m.avg

Channels: Ch1 Ch2 Ch3 Ch4 Ch5 Ch3 Ch4

Plotted: 09:30 Aug 18, 2004

< EMI - ElectroMagnetic Instruments > 


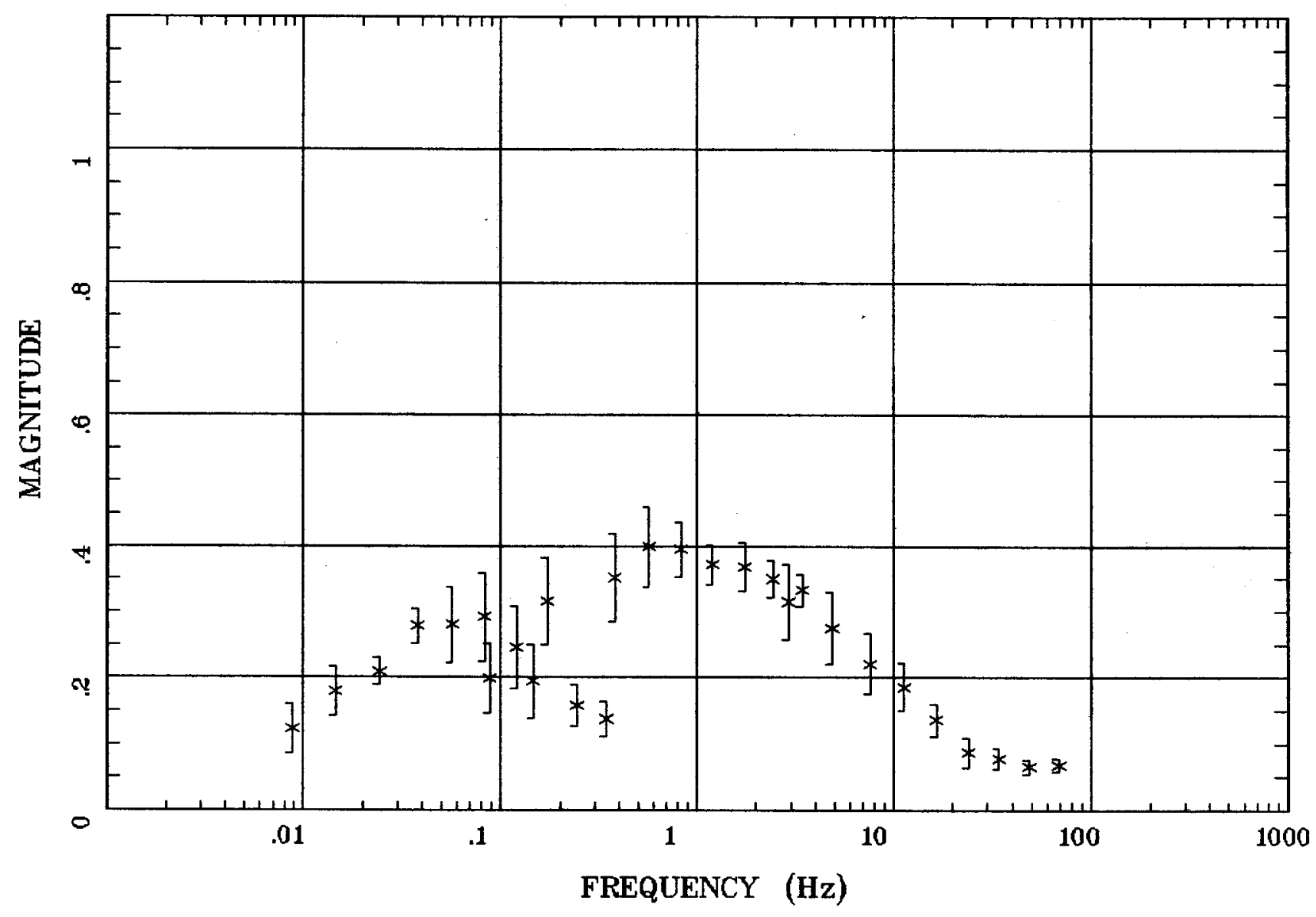

Client: Espanola Basin

Remote: none

Acquired: 11:1 Jun 08, 2004

Survey Co:USGS
Rotation:

Filename: cp35m.avg

Channels: Ch1 Ch2 Ch3 Ch4 Ch5 Ch3 Ch4

Plotted: 09:30 Aug 18, 2004

< EMI - ElectroMagnetic Instruments 


\section{Station 35}

TIPPER STRIKE

Los Alamos, NM 100k

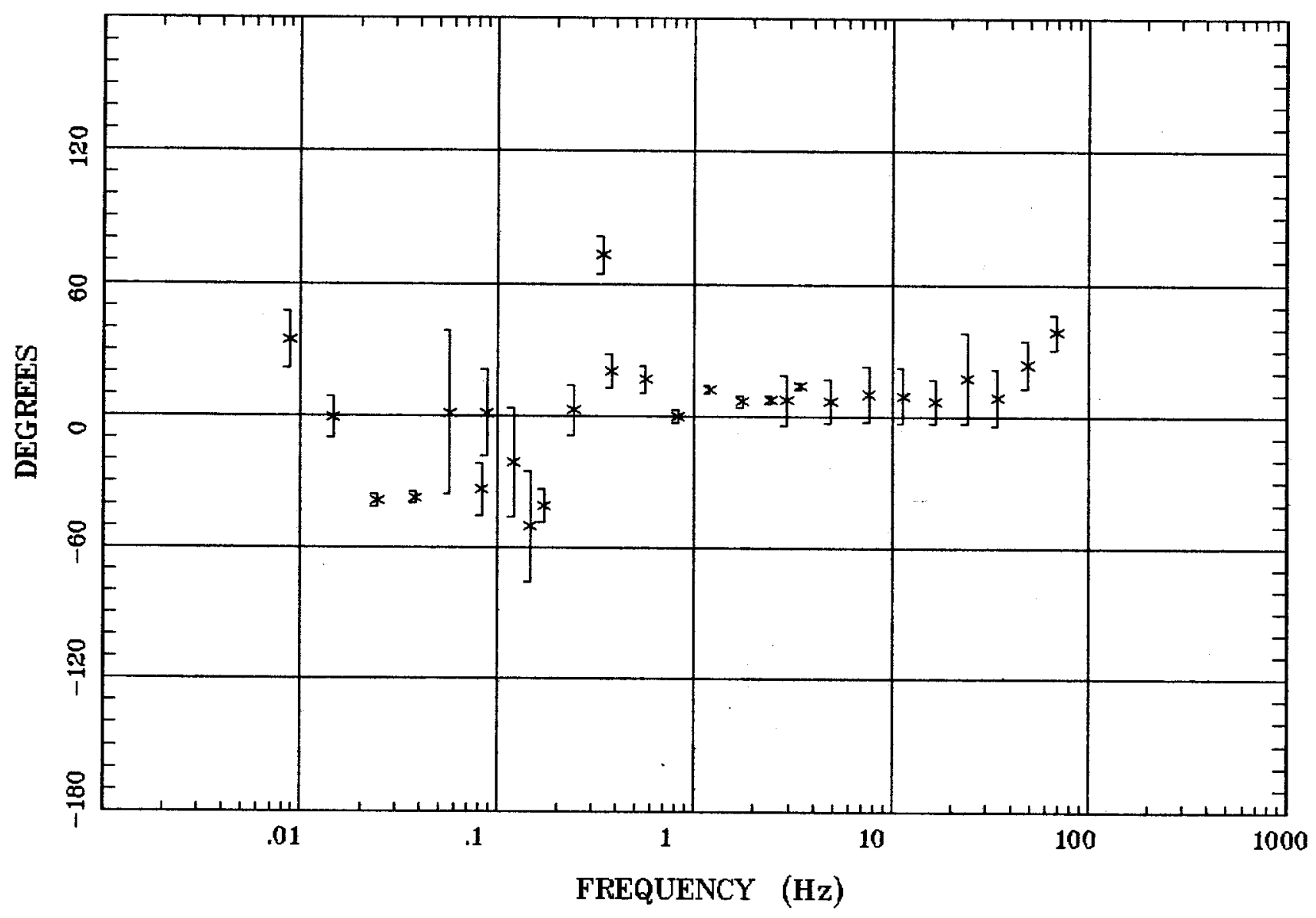

Client: Espanola Basin Remote: none Acquired: 11:1 Jun 08, 2004 Survey Co:USGS
Rotation:

Filename: cp35m.avg

Channels: Ch1 Ch2 Ch3 Ch4 Ch5 Ch3 Ch4 Plotted: 09:30 Aug 18, 2004

< EMI - ElectroMagnetic Instruments > 
HzHx.x Coh HzHy.o

Los Alamos, NM 100k

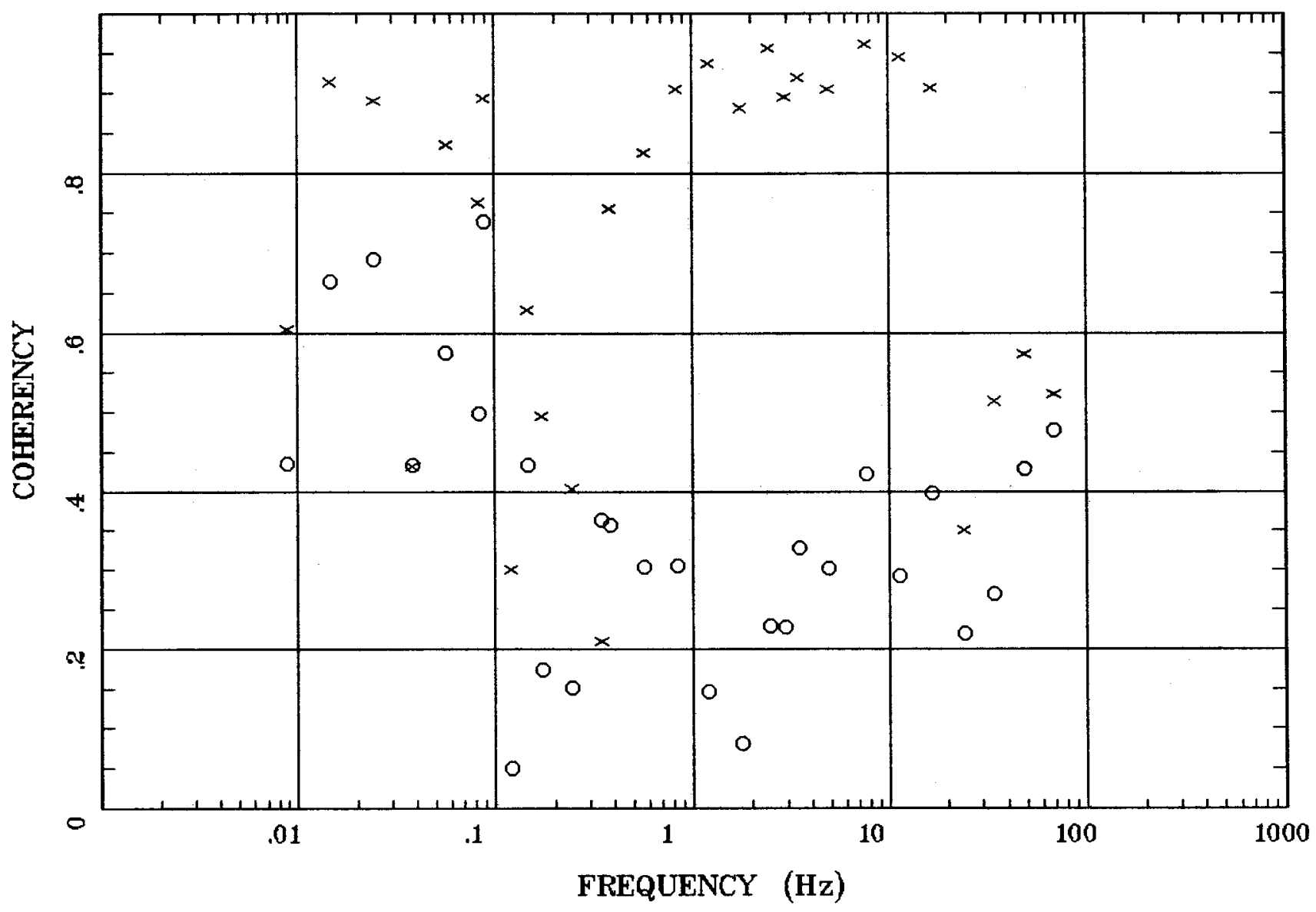

Client: Espanola Basin

Remote: none

Acquired: 11:1 Jun 08, 2004

Survey Co:USGS
Rotation:

Filename: cp35m.avg

Channels: Ch1 Ch2 Ch3 Ch4 Ch5 Ch3 Ch4 Plotted: 09:30 Aug 18, 2004

$<$ EMI - ElectroMagnetic Instruments 


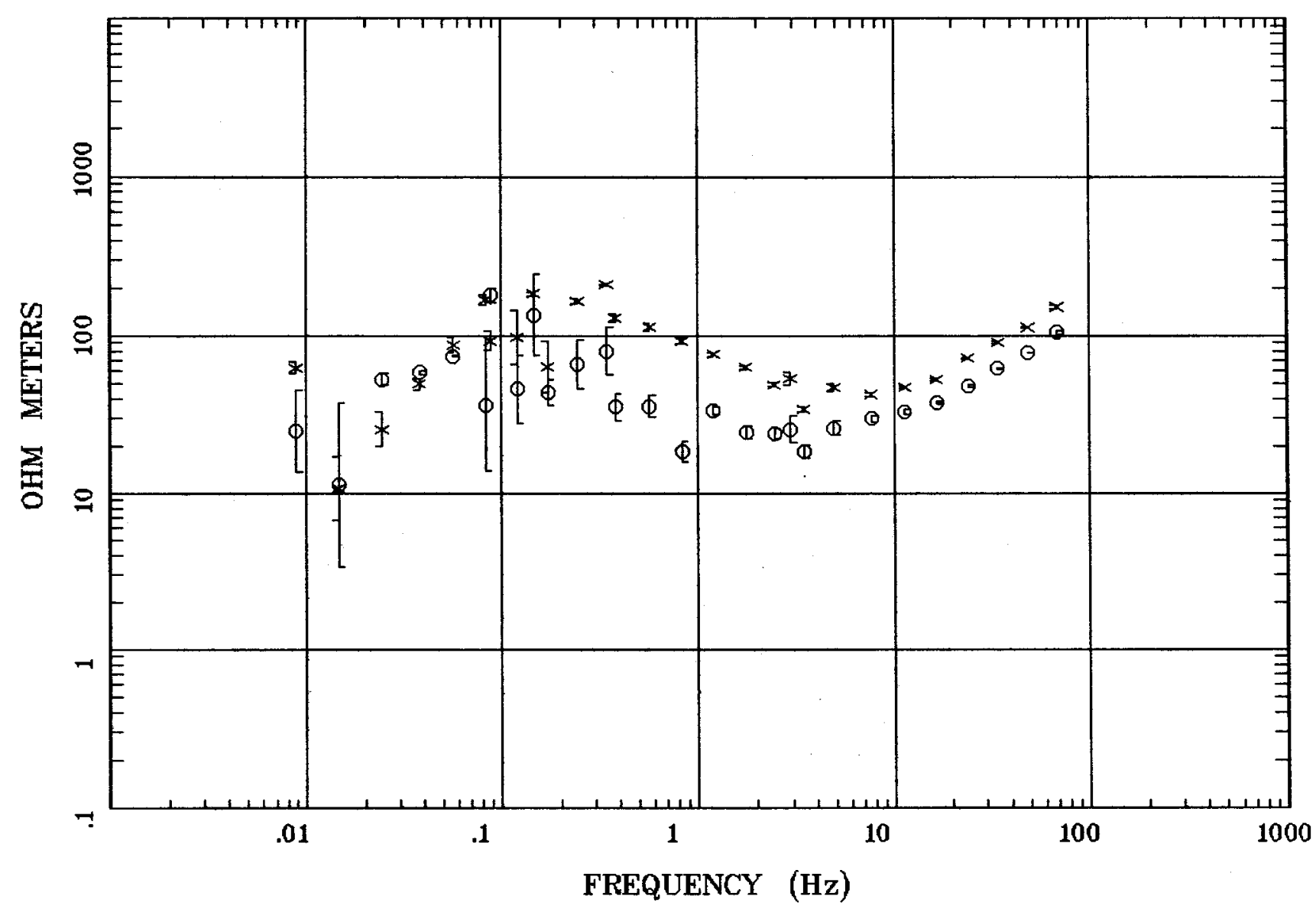

Client: Espanola Basin

Remote: none

Acquired: 10:1 Jul 16, 2004

Survey Co:USGS
Rotation:

Filename: cp34m.avg

Channels: Ch1 Ch2 Ch3 Ch4 Ch5 Ch3 Ch4

Plotted: 14:02 Aug 18, 2004

< EMI - ElectroMagnetic Instruments > 


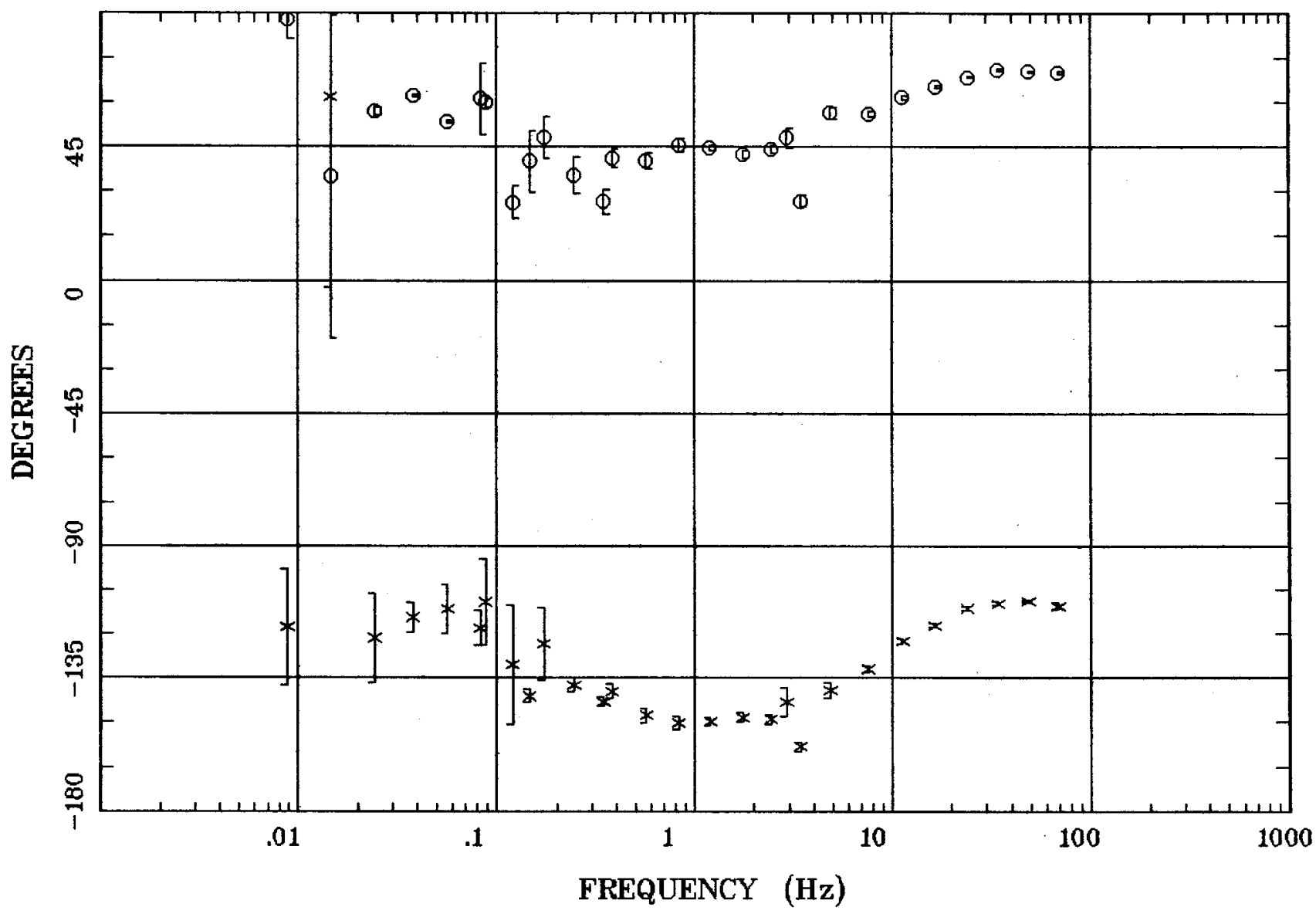

Client: Espanola Basin Remote: none Acquired: 10:1 Jul 16, 2004 Survey Co:USGS
Rotation:

Filename: cp34m.avg Channels: Ch1 Ch2 Ch3 Ch4 Ch5 Ch3 Ch4 Plotted: 14:03 Aug 18, 2004

< EMI - ElectroMagnetic Instruments > 


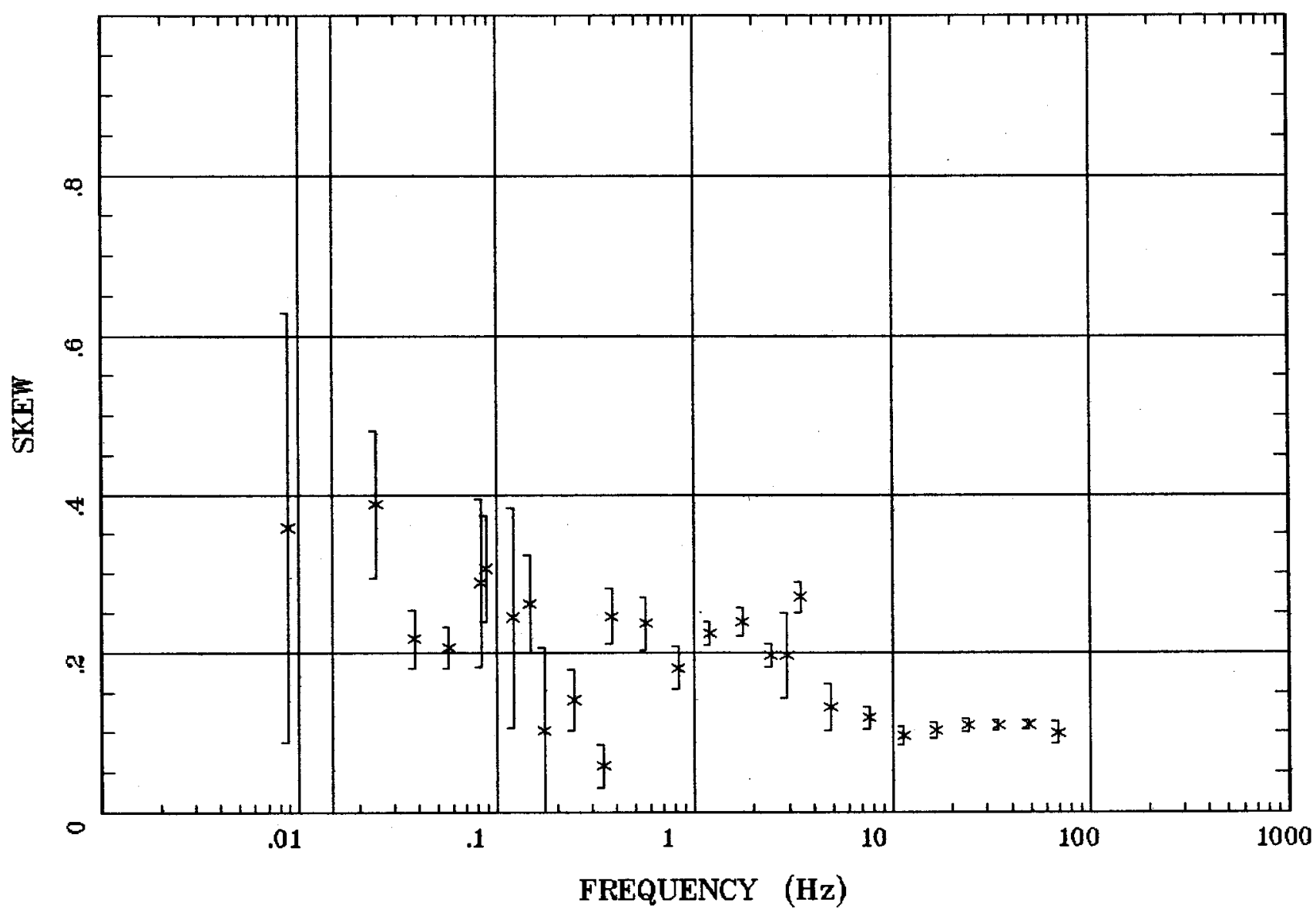

Client: Espanola Basin Remote: none Acquired: 10:1 Jul 16, 2004 Survey Co:USGS
Rotation:

Filename: cp34m.avg

Channels: Ch1 Ch2 Ch3 Ch4 Ch5 Ch3 Ch4 Plotted: 14:03 Aug 18, 2004

< EMI - ElectroMagnetic Instruments 
E MULT Coh.

Los Alamos, NM 100k

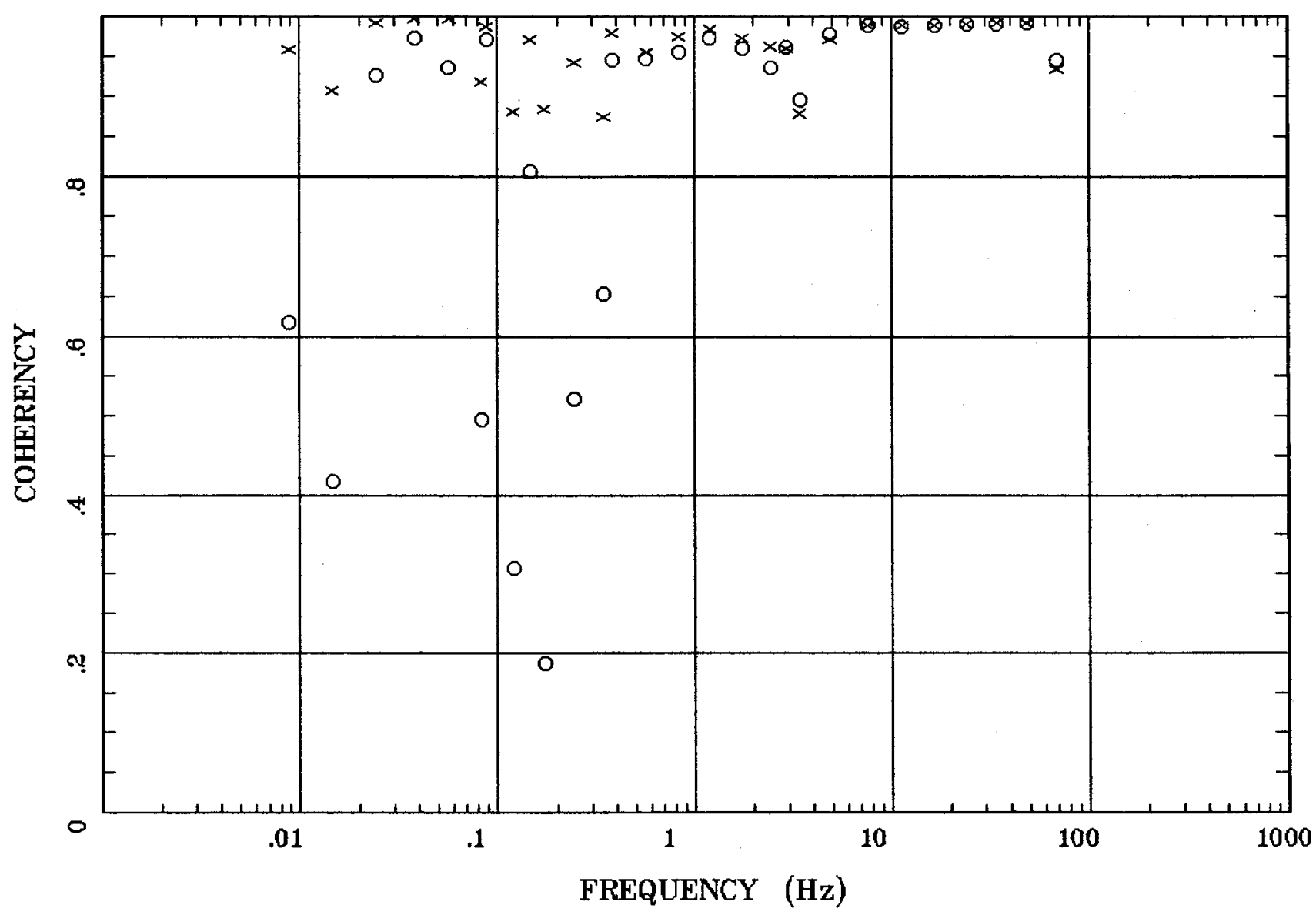

Client: Espanola Basin

Remote: none

Acquired: 10:1 Jul 16, 2004

Survey Co:USGS
Rotation:

Filename: cp34m.avg

Channels: Ch1 Ch2 Ch3 Ch4 Ch5 Ch3 Ch4

Platted: 14:03 Aug 18, 2004

$\langle$ EMI - ElectroMagnetic Instruments > 


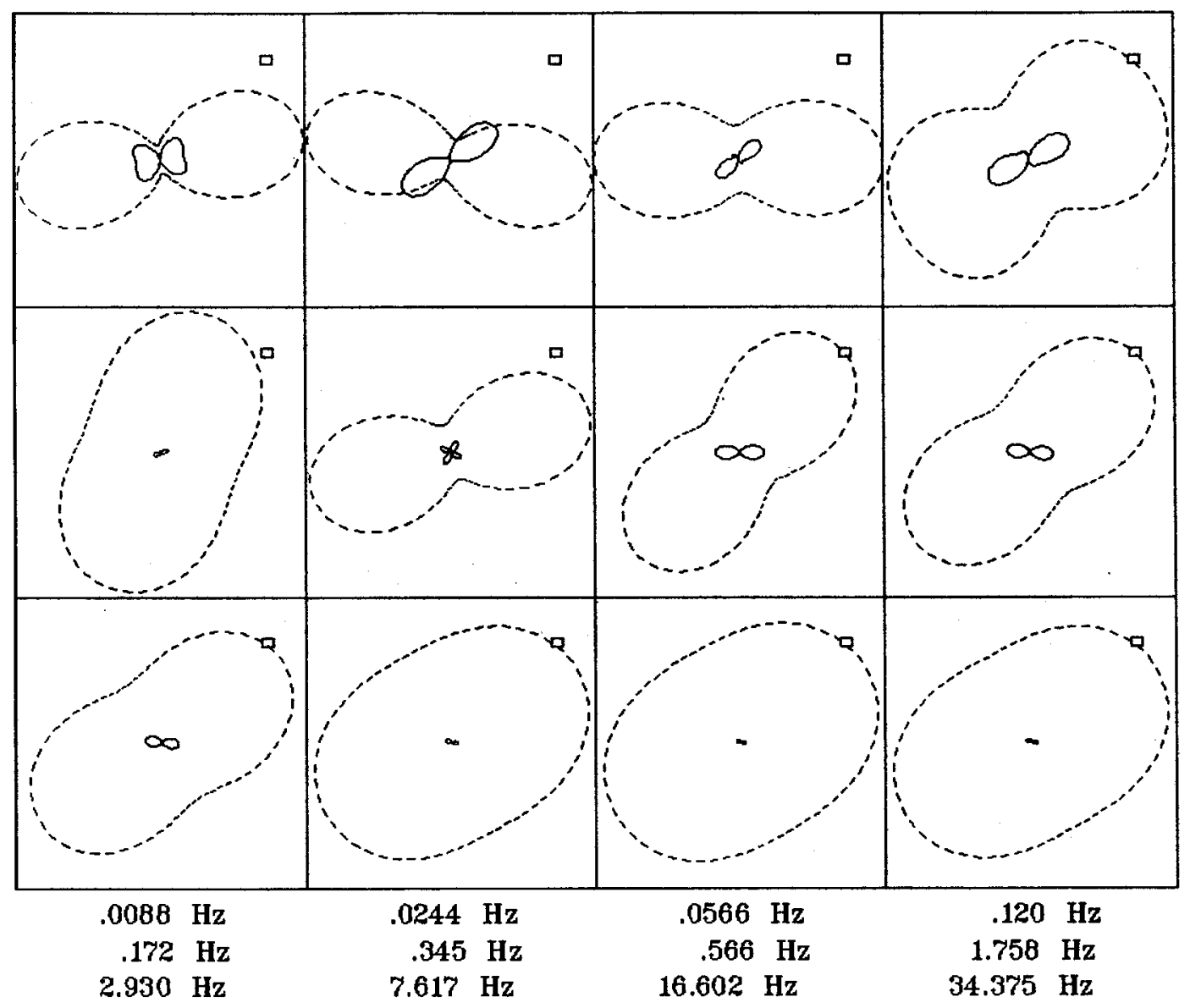

Client: Espanola Basin

Remote: none

Acquired: 10:1 Jul 16, 2004

Survey Co:USGS
Rotation:

Filename: cp34m.avg

Channels: Ch1 Ch2 Ch3 Ch4 Ch5 Ch3 Ch4

Plotted: 14:03 Aug 18, 2004

< EMI - ElectroMagnetic Instruments > 


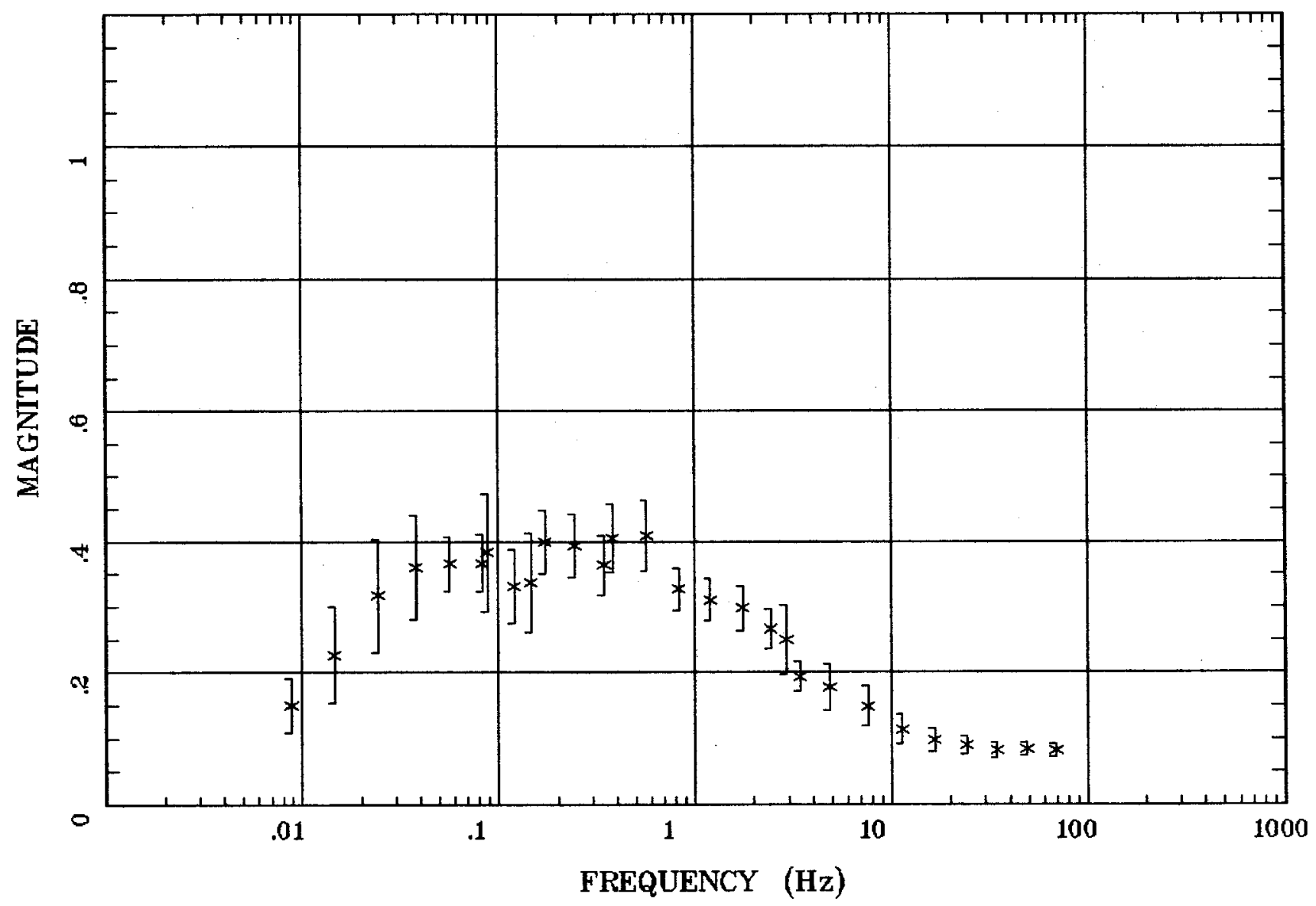

Client: Espanola Basin

Remote: none

Acquired: 10:1 Jul 16, 2004

Survey Ca:USGS
Rotation:

Filename: cp34m.avg

Channels: Ch1 Ch2 Ch3 Ch4 Ch5 Ch3 Ch4 Plotted: 14:03 Aug 18, 2004

< EMI - ElectroMagnetic Instruments 


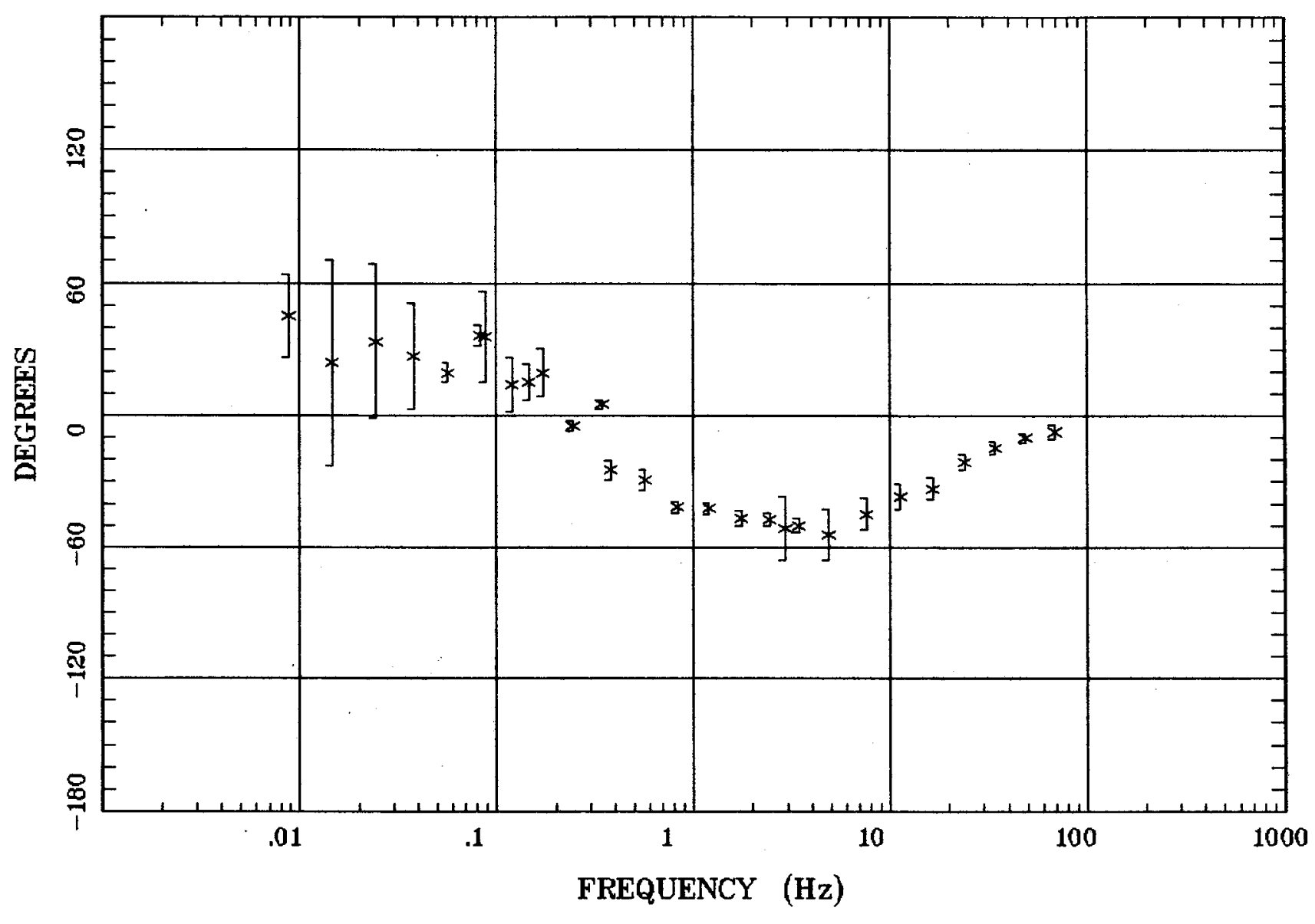

Client: Espanola Basin Remote: none Acquired: 10:1 Jul 16, 2004 Survey Co:USGS
Rotation:

Filename: cp34m.avg

Channels: Ch1 Ch2 Ch3 Ch4 Ch5 Ch3 Ch4 Plotted: 14:03 Aug 18, 2004

$<$ EMI - ElectroMagnetic Instruments 


\section{HzHx.x Coh HzHy.o}

Los Alamos, NM 100k

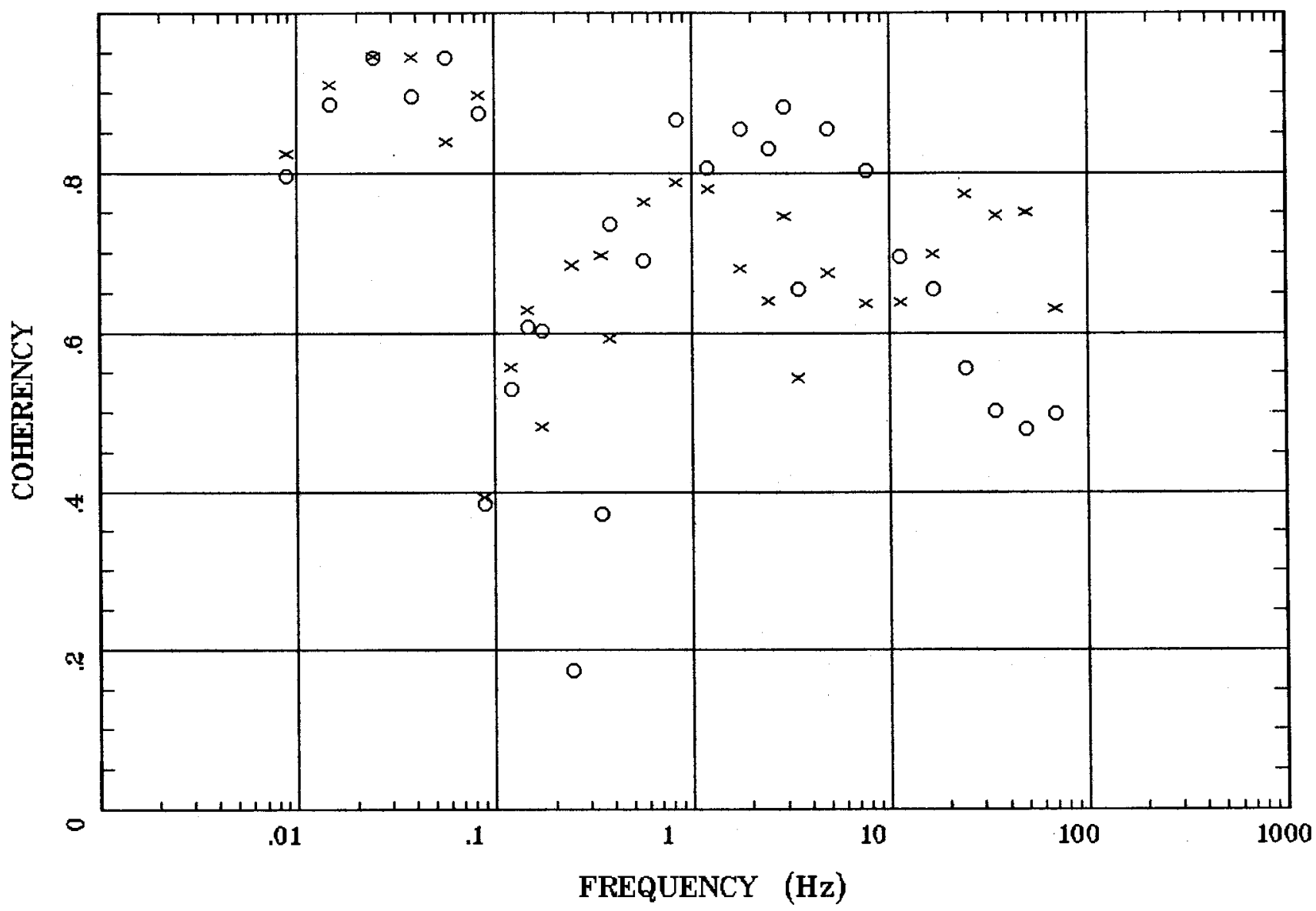

Client: Espanola Basin Remote: none Acquired: 10:1 Jul 16, 2004 Survey Co:USGS
Rotation:

Filename: cp34m.avg Channels: Ch1 Ch2 Ch3 Ch4 Ch5 Ch3 Ch4 Plotted: 14:03 Aug 18, 2004

< EMI - ElectroMagnetic Instruments 
Station 33

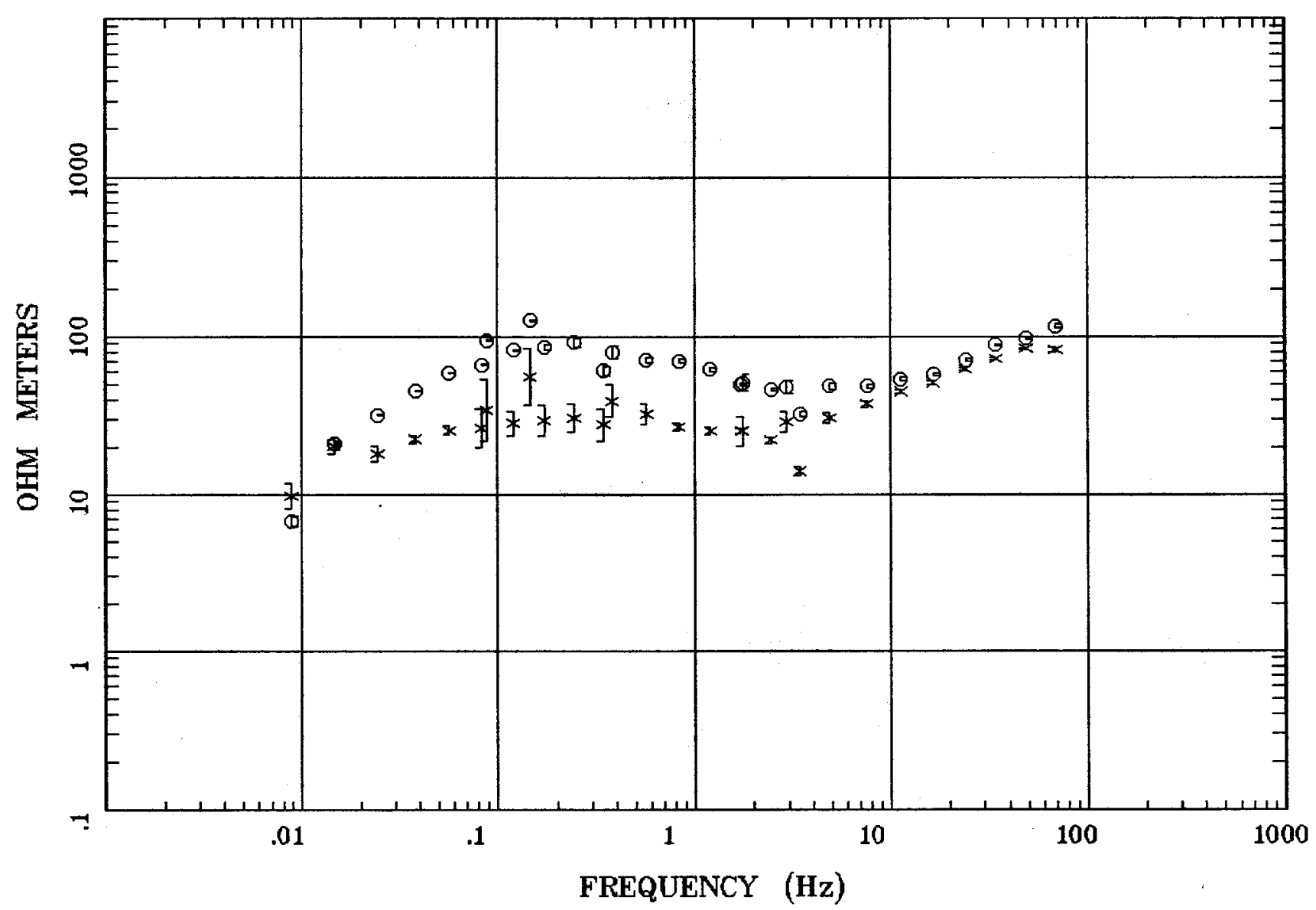

Client: Espanola Basin Remote: none

Acquired: 13:2 Jul 13, 2004 Survey Co:USGS

\section{Rotation:}

Filename: cp33mall.avg

Channels: Ch1 Ch2 Ch3 Ch4 Ch5 Ch3 Ch4 Plotted: 14:09 Aug 18, 2004

< EMI - ElectroMagnetic Instruments > 


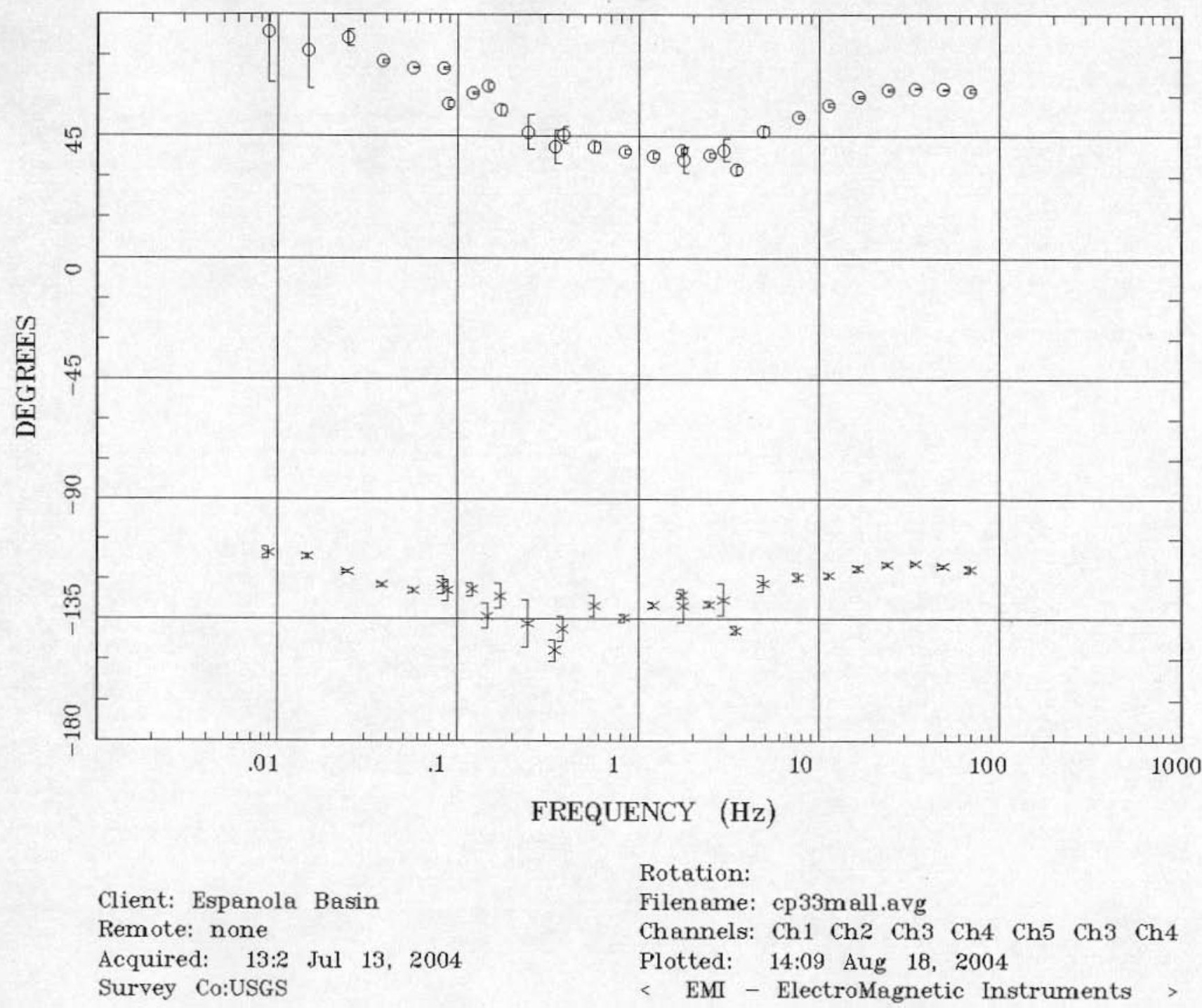




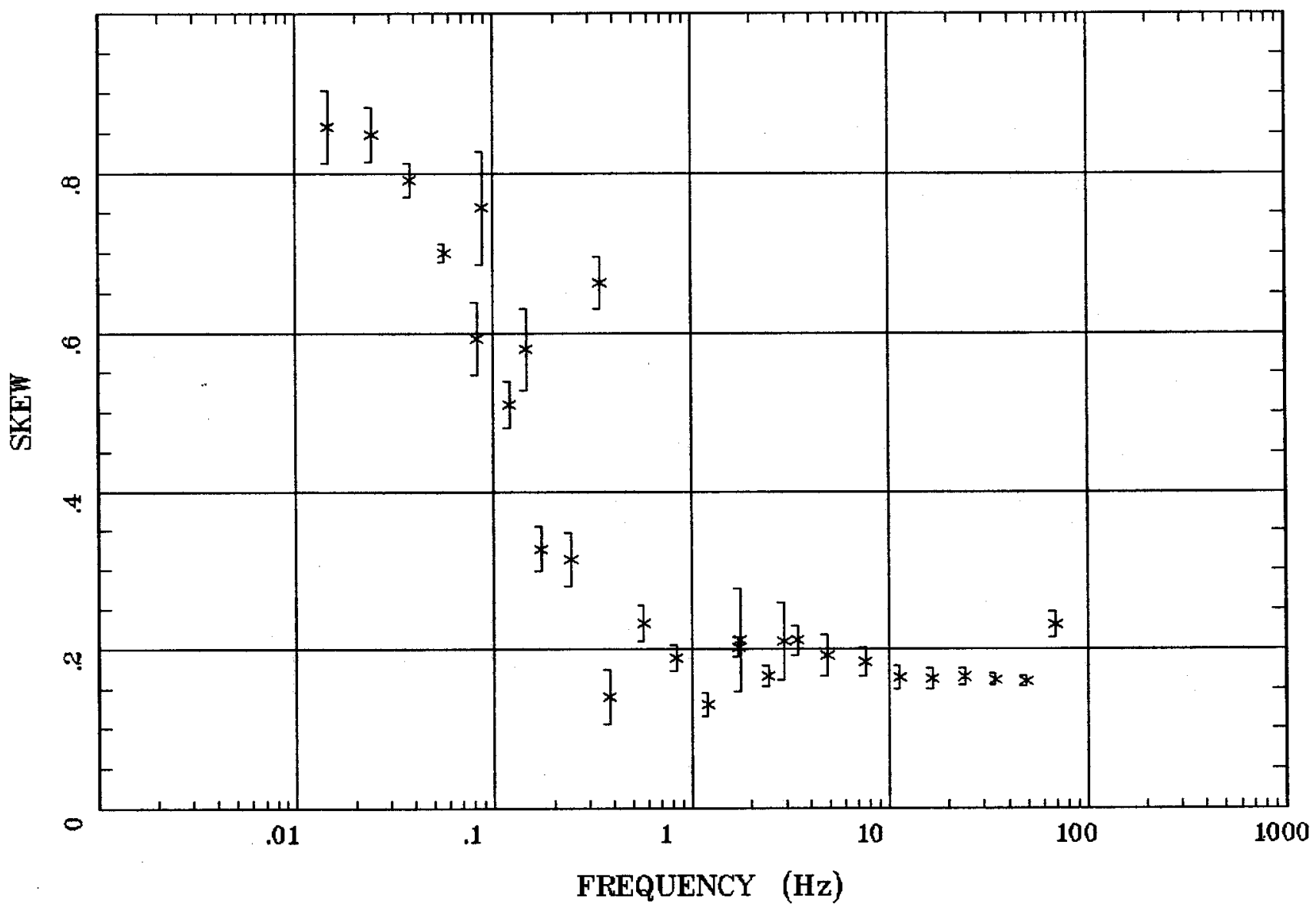

Client: Espanola Basin

Remote: none

Acquired: 13:2 Jul 13, 2004 Survey Co:USGS
Rotation:

Filename: cp33mall.avg

Channels: Ch1 Ch2 Ch3 Ch4 Ch5 Ch3 Ch4

Plotted: 14:09 Aug 18, 2004

< EMI - ElectroMagnetic Instruments 
E MULT Coh.

Los Alamos, NM 100k

Station 33

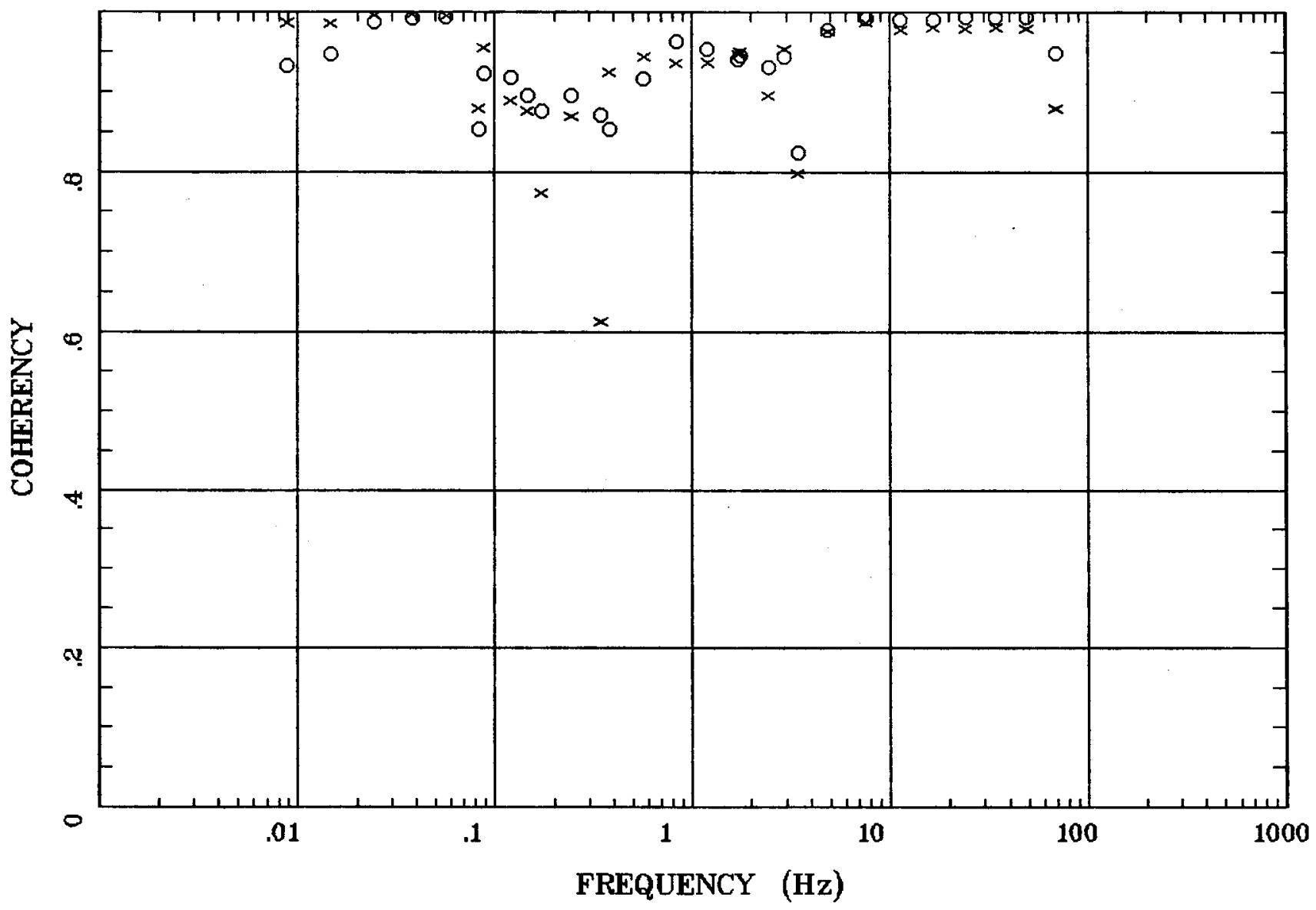

Client: Espanola Basin Remote: none Acquired: 13:2 Jul 13, 2004 Survey Co:USGS
Rotation:

Filename: cp33mall.avg

Channels: Ch1 Ch2 Ch3 Ch4 Ch5 Ch3 Ch4 Plotted: 14:09 Aug 18, 2004

< EMI - ElectroMagnetic Instruments 


\section{POLAR PLOTS}

Los Alamos, NM 100k

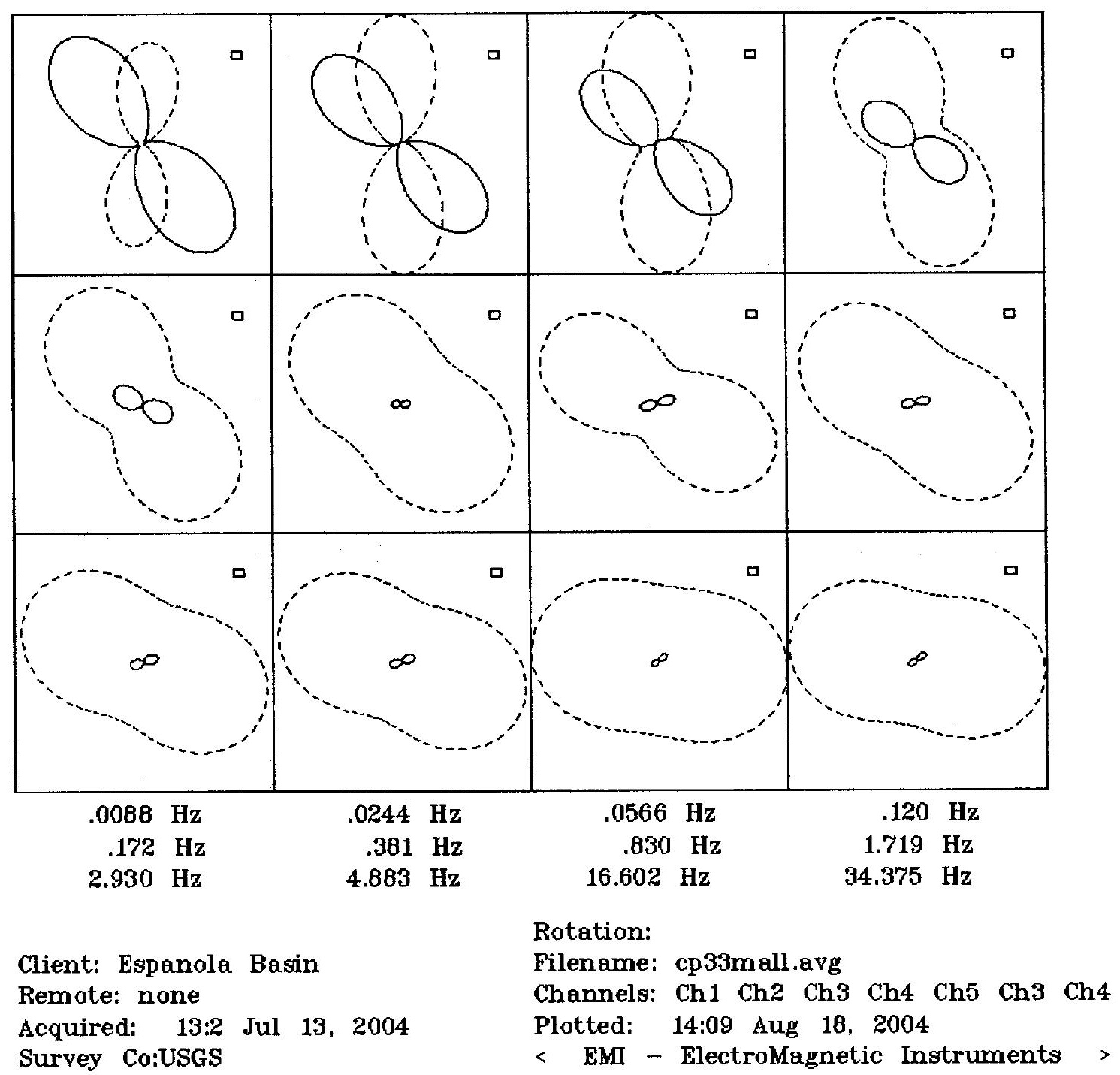




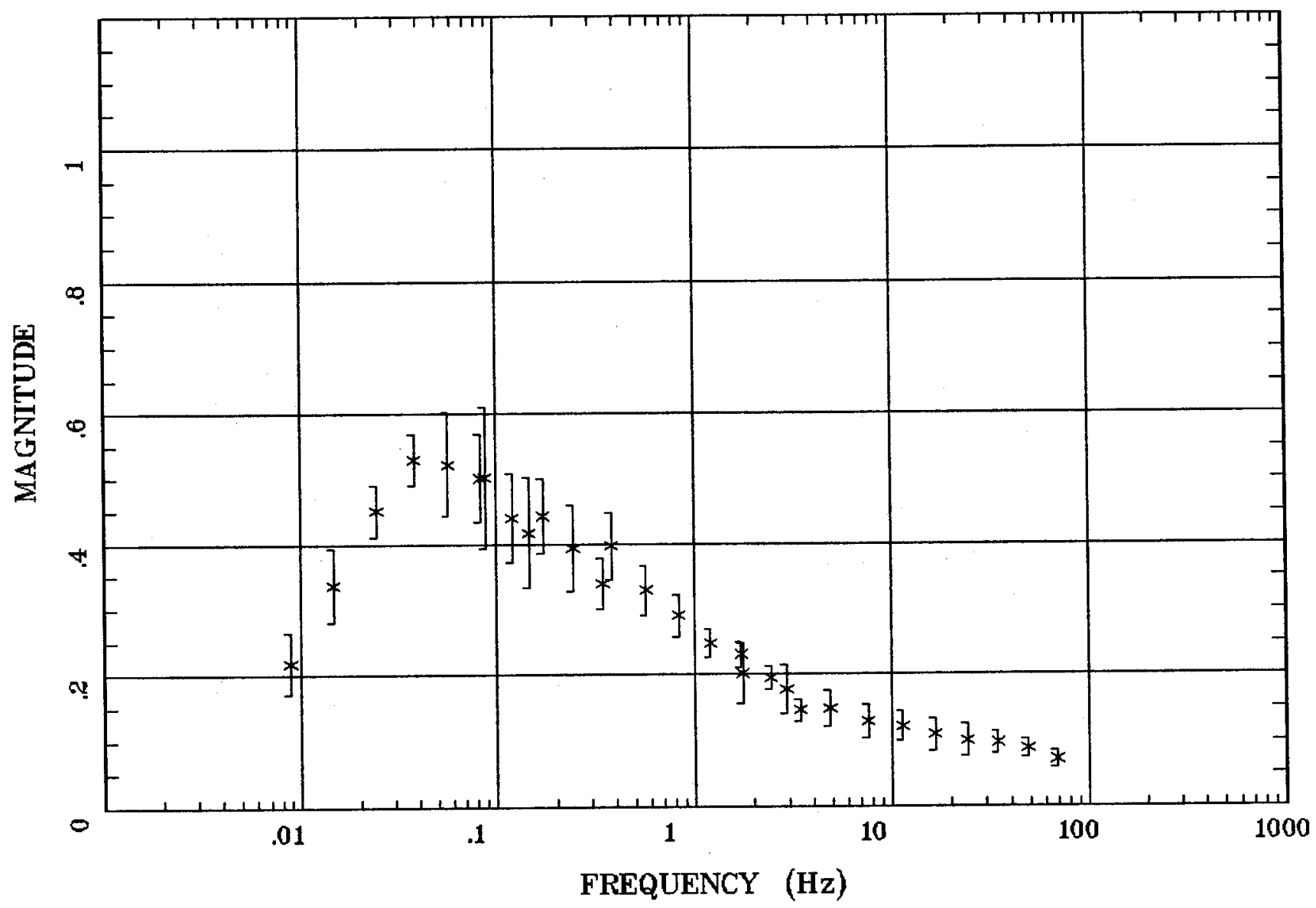

Client: Espanola Basin

Remote: none

Acquired: 13:2 Jul 13, 2004

Survey Co:USGS
Rotation:

Filename: cp33mall.avg

Channels: Ch1 Ch2 Ch3 Ch4 Ch5 Ch3 Ch4

Plotted: 14:09 Aug 18, 2004

< EMI - ElectroMagnetic Instruments > 
Station 33

TIPPER STRIKE

Los Alamos, NM 100k

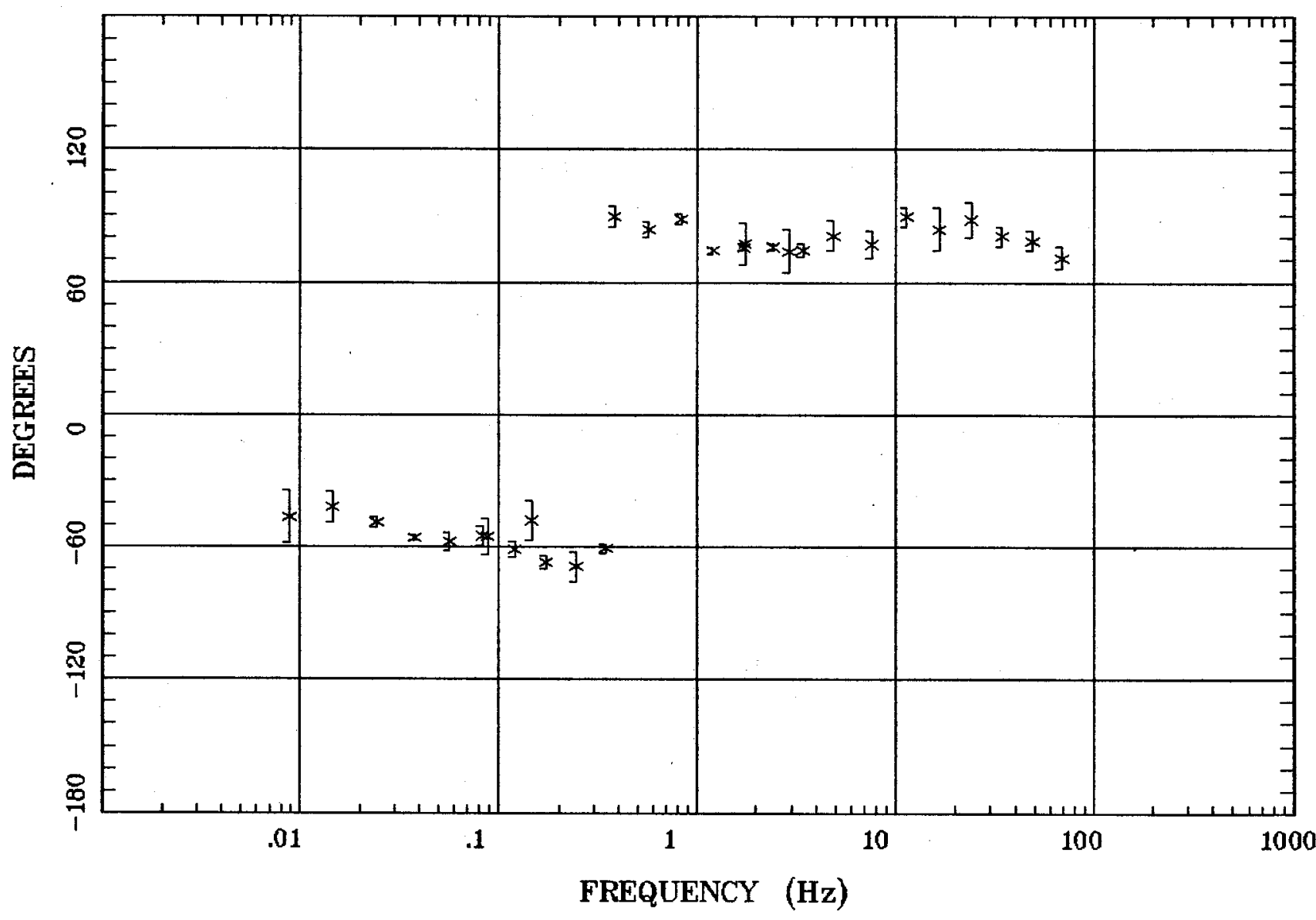

Client: Espanola Basin

Remote: none

Acquired: 13:2 Jul 13, 2004

Survey Co:USGS
Rotation:

Filename: cp33mall.avg

Channels: Ch1 Ch2 Ch3 Ch4 Ch5 Ch3 Ch4 Platted: 14:09 Aug 18, 2004

< EMI - ElectroMagnetic Instruments > 
HzHx.x Coh HzHy.o

Los Alamos, NM 100k

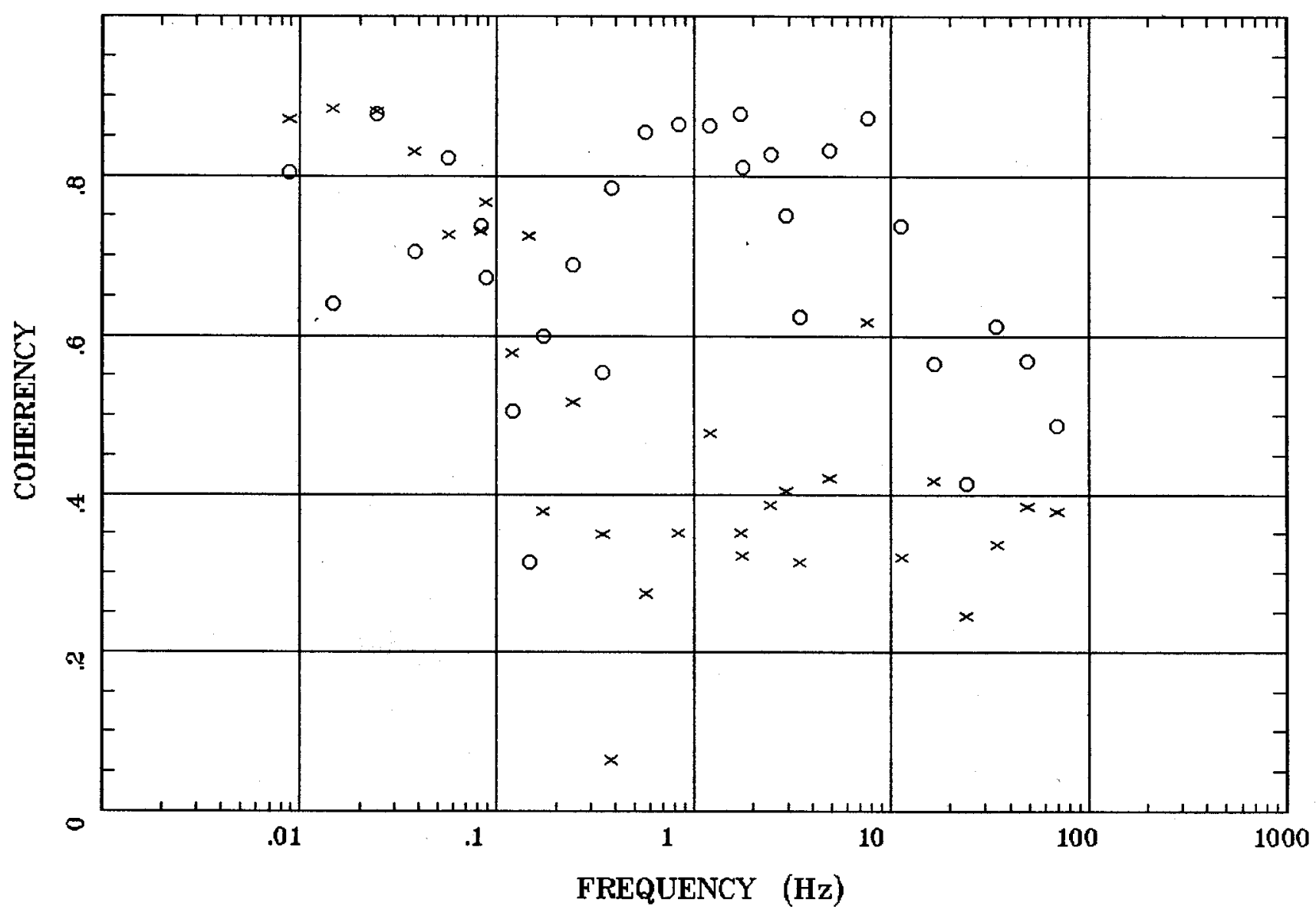

Client: Espanola Basin

Remote: none

Acquired: 13:2 Jul 13, 2004

Survey Co:USGS
Rotation:

Filename: cp33mall.avg

Channels: Ch1 Ch2 Ch3 Ch4 Ch5 Ch3 Ch4

Plotted: 14:09 Aug 18, 2004

$<$ EMI - ElectroMagnetic Instruments 


\section{APPARENT RESISTIVITY}

Los Alamos, NM 100k

Station 32

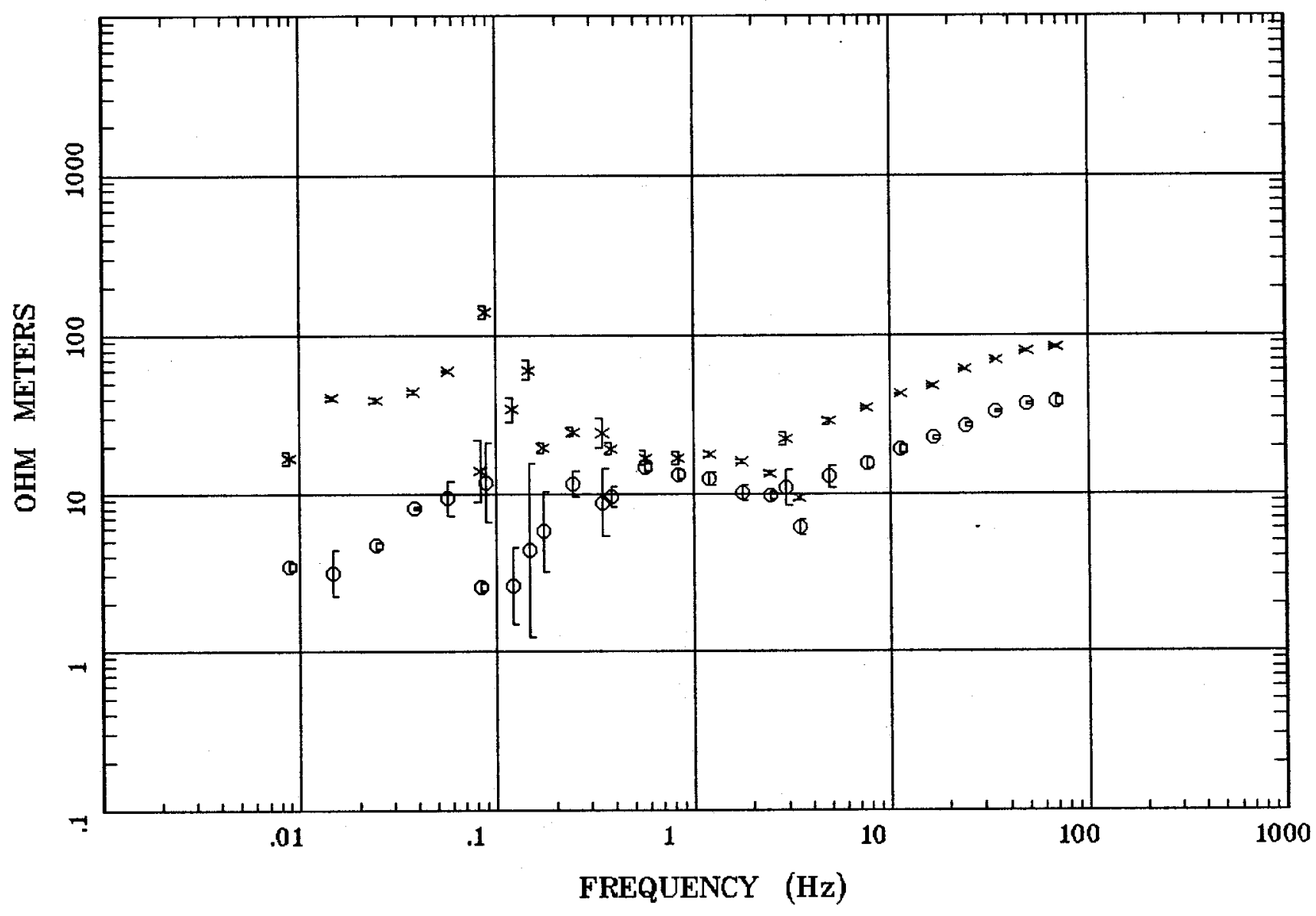

Client: Espanola Basin

Remote: none

Acquired: 10:4 Jul 15, 2004

Survey Co:USGS
Rotation:

Filename: cp32m.avg

Channels: Ch1 Ch2 Ch3 Ch4 Ch5 Ch3 Ch4

Plotted: 14:04 Aug 18, 2004

$<$ EMI - ElectroMagnetic Instruments > 


\section{IMPEDANCE PHASE}

Los Alamos, NM 100k

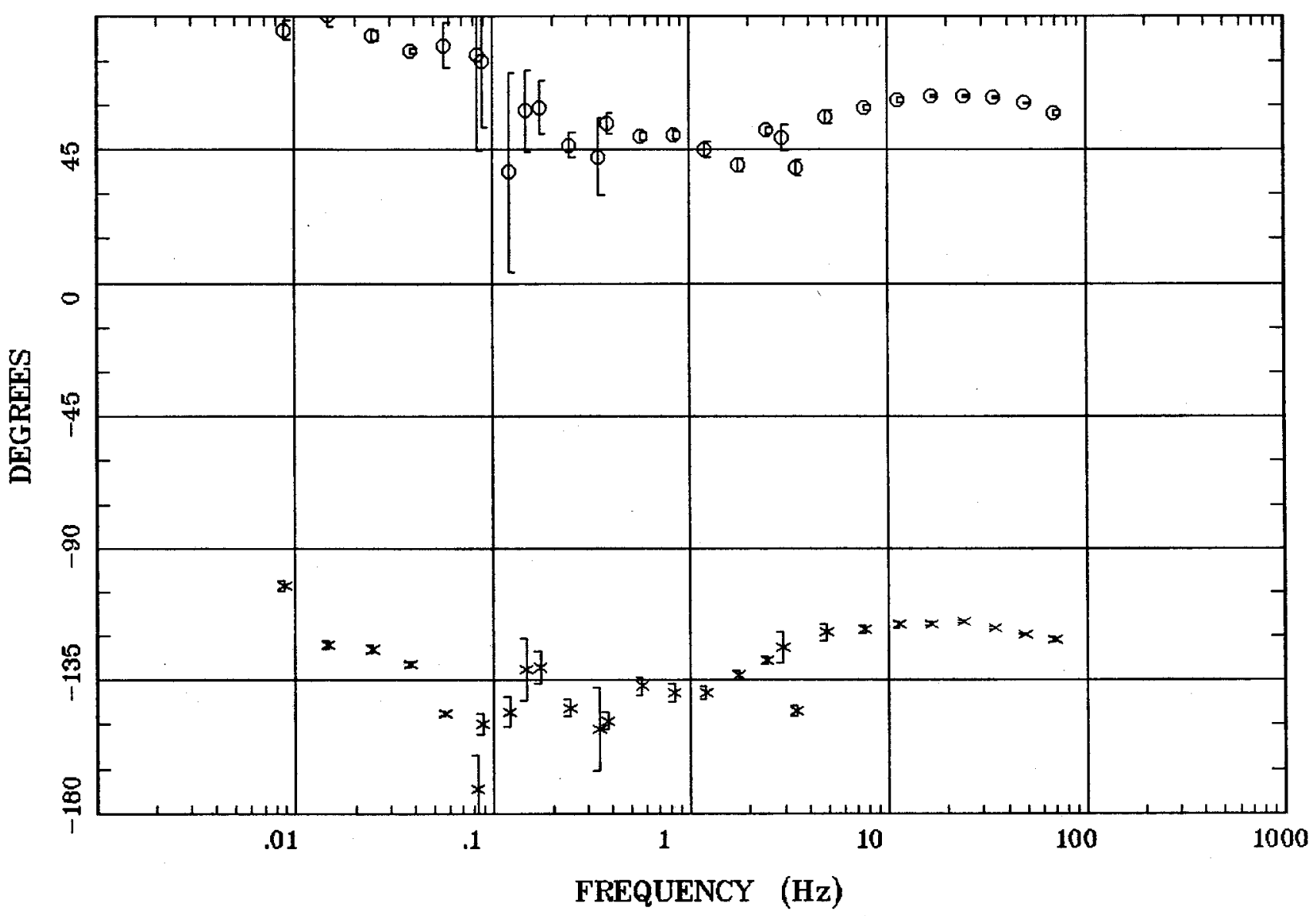

Client: Espanola Basin

Remote: none

Acquired: 10:4 Jul 15, 2004

Survey Co:USGS
Rotation:

Filename: cp32m.avg

Channels: Ch1 Ch2 Ch3 Ch4 Ch5 Ch3 Ch4

Plotted: 14:04 Aug 18, 2004

$<$ EMI - ElectroMagnetic Instruments 
Los Alamos, NM 100k

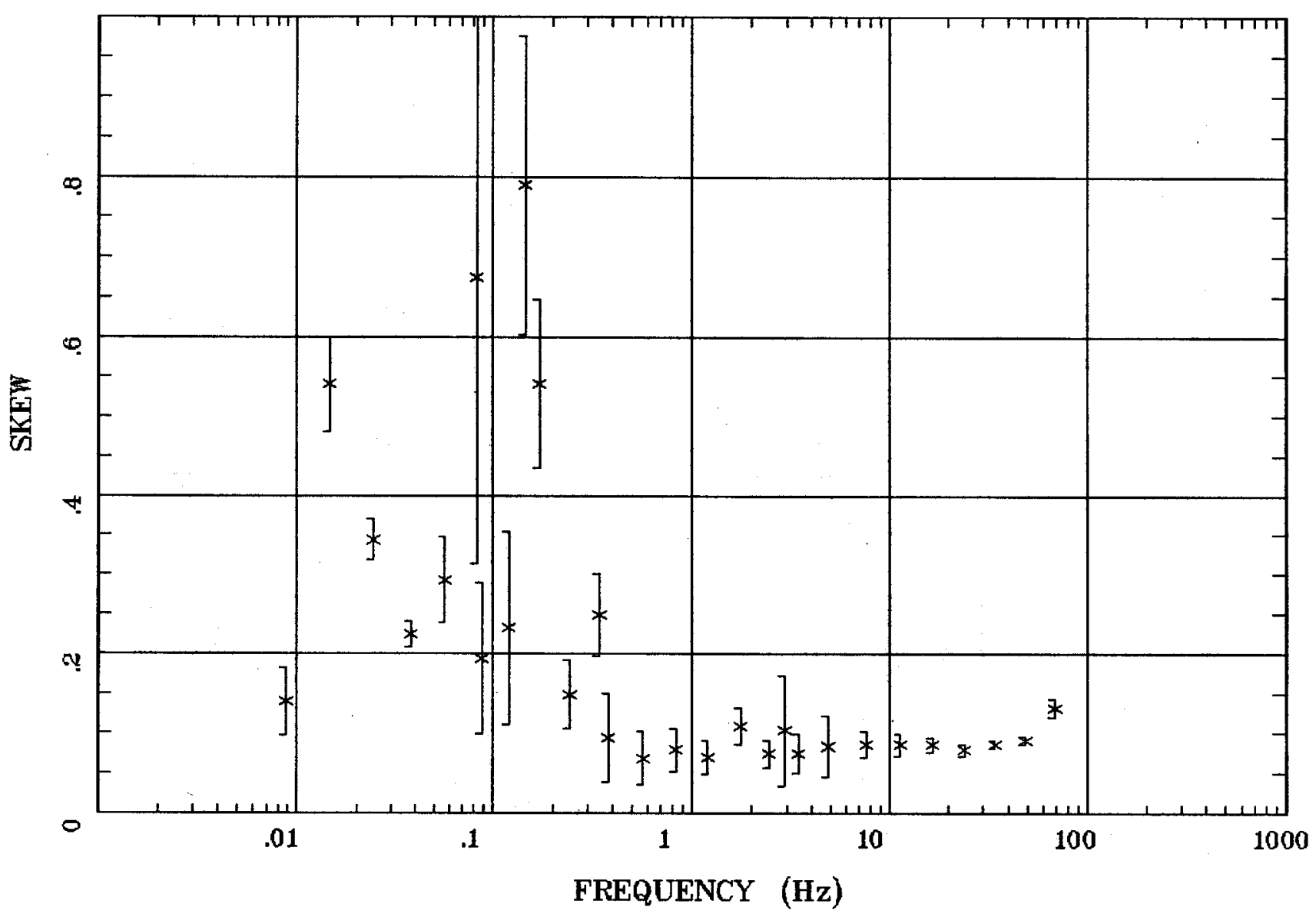

Client: Espanola Basin

Remote: none

Acquired: 10:4 Jul 15, 2004 Survey Co:USGS
Rotation:

Filename: cp32m.avg

Channels: Ch1 Ch2 Ch3 Ch4 Ch5 Ch3 Ch4

Platted: 14:04 Aug 18, 2004

$<$ EMI - ElectroMagnetic Instruments 
E MULT Coh.

Los Alamos, NM 100k

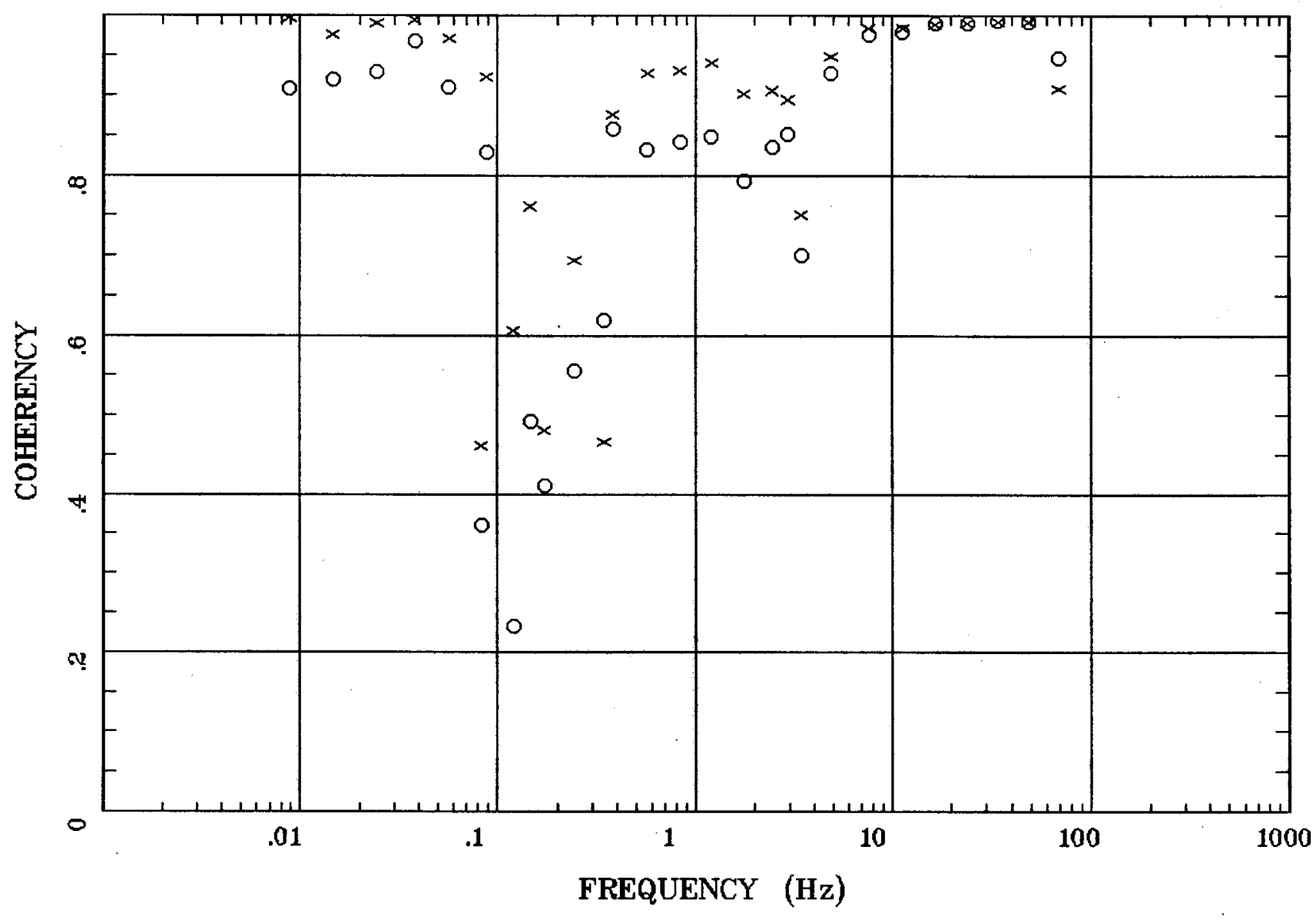

Client: Espanola Basin Remote: none Acquired: 10:4 Jul 15, 2004 Survey Co:USGS
Rotation:

Filename: cp32m.avg

Channels: Ch1 Ch2 Ch3 Ch4 Ch5 Ch3 Ch4 Plotted: 14:04 Aug 18, 2004

$<$ EMI - ElectroMagnetic Instruments 


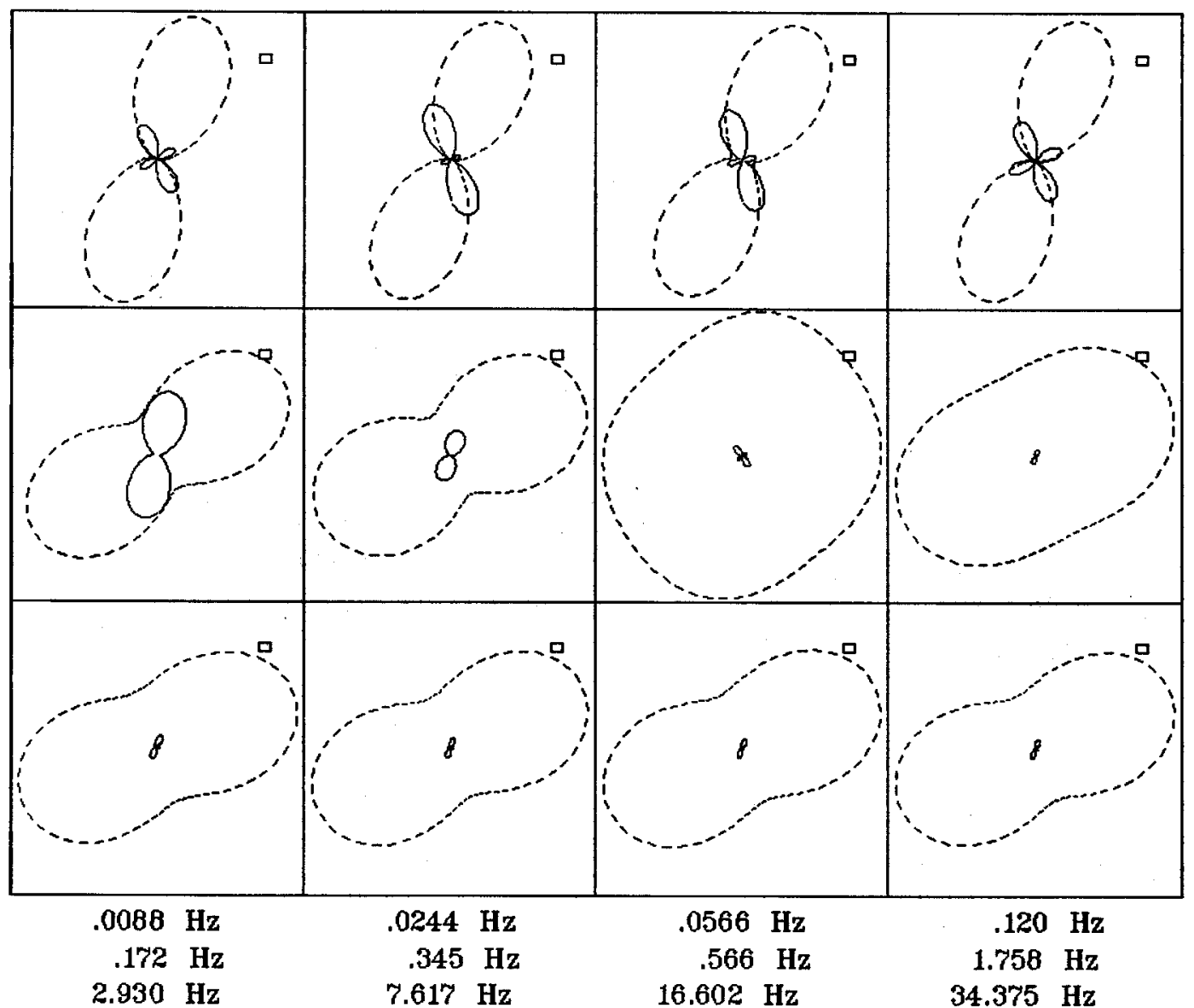

Client: Espanola Basin Remote: none

Acquired: 10:4 Jul 15, 2004 Survey Co:USGS
Rotation:

Filename: cp32m.avg

Channels: Ch1 Ch2 Ch3 Ch4 Ch5 Ch3 Ch4 Plotted: 14:04 Aug 18, 2004

< EMI - ElectroMagnetic Instruments 


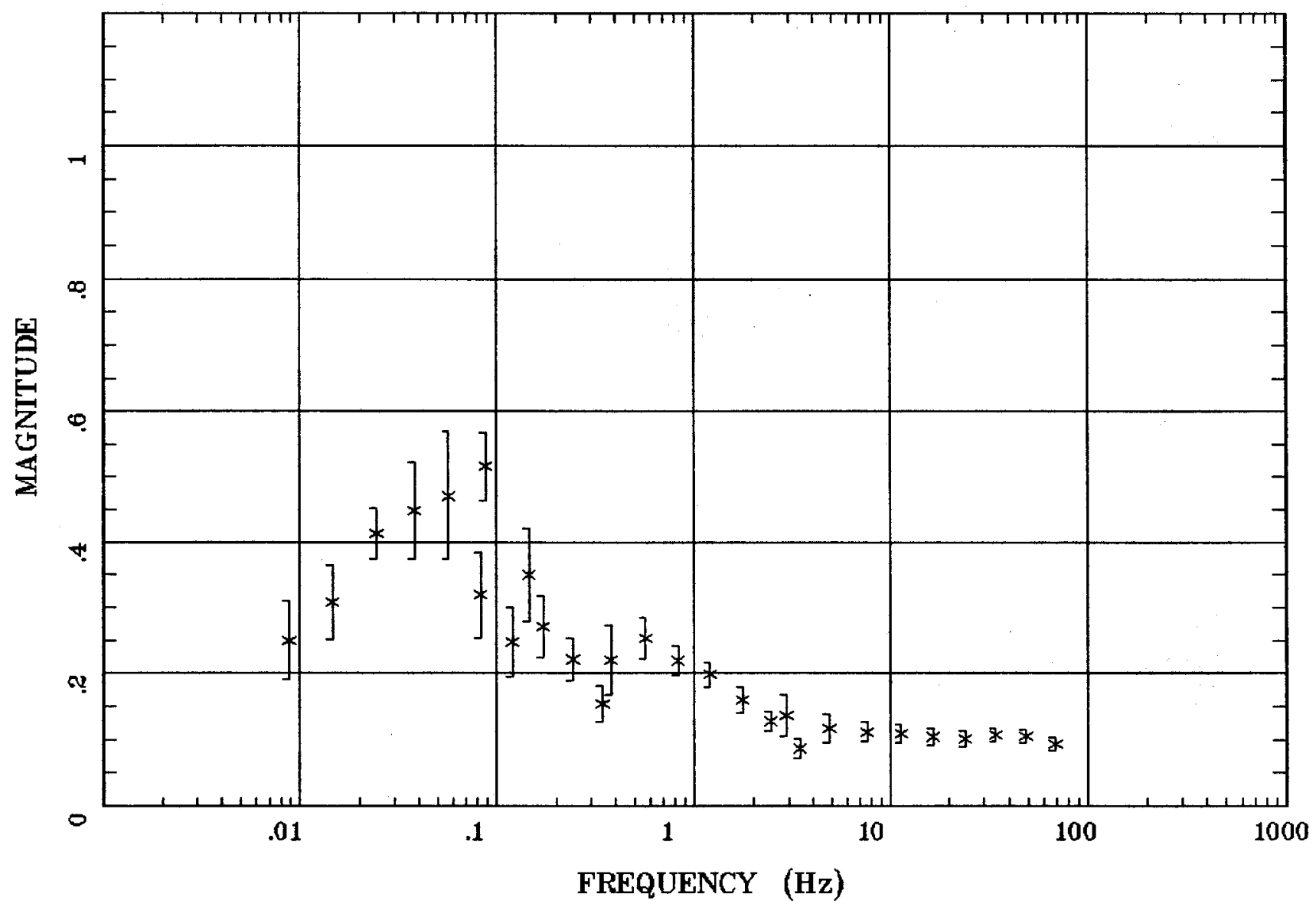

Client: Espanola Basin

Remate: none

Acquired: 10:4 Jul 15, 2004

Survey Co:USGS

\section{Rotation:}

Filename: cp32m.avg

Channels: Ch1 Ch2 Ch3 Ch4 Ch5 Ch3 Ch4 Plotted: 14:04 Aug 18, 2004.

$<$ EMI - ElectroMagnetic Instruments 


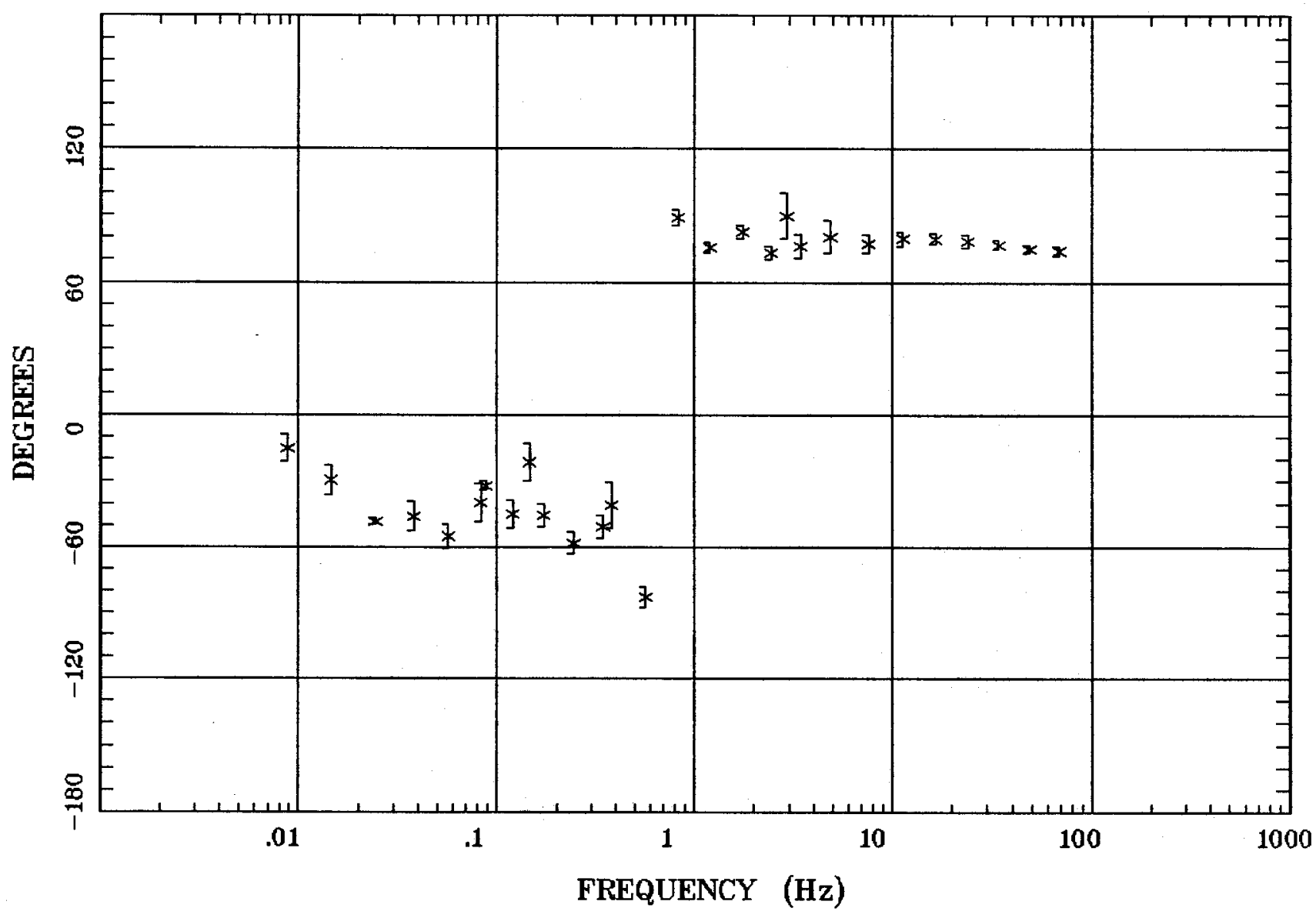

Client: Espanala Basin Remote: none Acquired: 10:4 Jul 15, 2004 Survey Co:USGS
Rotation:

Filename: cp32m.avg

Channels: Ch1 Ch2 Ch3 Ch4 Ch5 Ch3 Ch4 Plotted: 14:04 Aug 18, 2004

< EMI - ElectroMagnetic Instruments 
HzHx.x Coh HzHy.o

Los Alamos, NM 100k

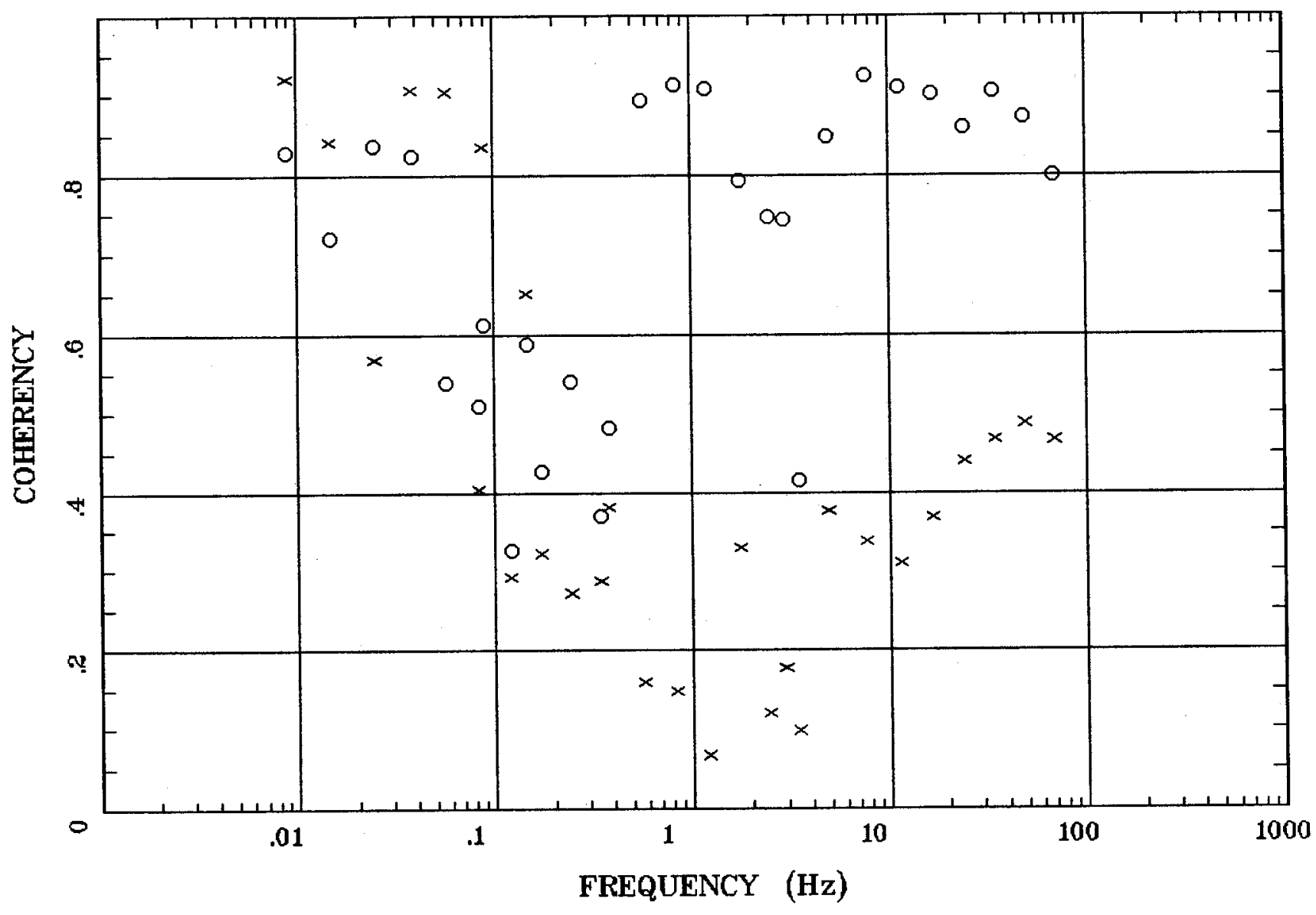

Client: Espanola Basin

Remote: none

Acquired: 10:4 Jul 15, 2004 Survey Co:USGS
Rotation:

Filename: cp32m.avg

Channels: Ch1 Ch2 Ch3 Ch4 Ch5 Ch3 Ch4

Plotted: 14:04 Aug 18, 2004

< EMI - ElectroMagnetic Instruments 


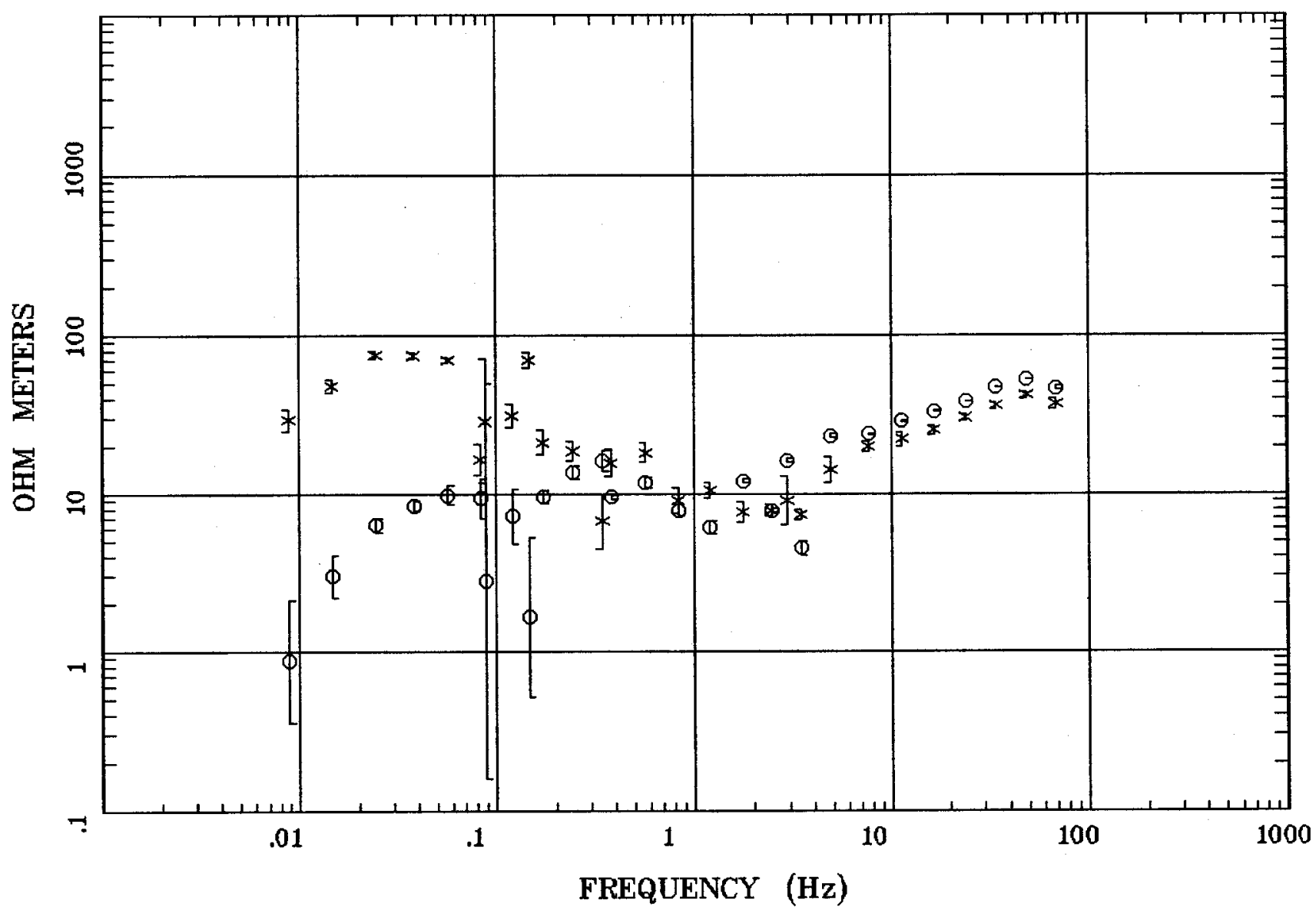

Client: Espanola Basin

Remote: none

Acquired: 11:1 Jul 14, 2004

Survey Co:USGS
Rotation:

Filename: cp31m68.avg

Channels: Ch1 Ch2 Ch3 Ch4 Ch5 Ch3 Ch4

Plotted: 14:01 Sep 01, 2004

< EMI - ElectroMagnetic Instruments > 


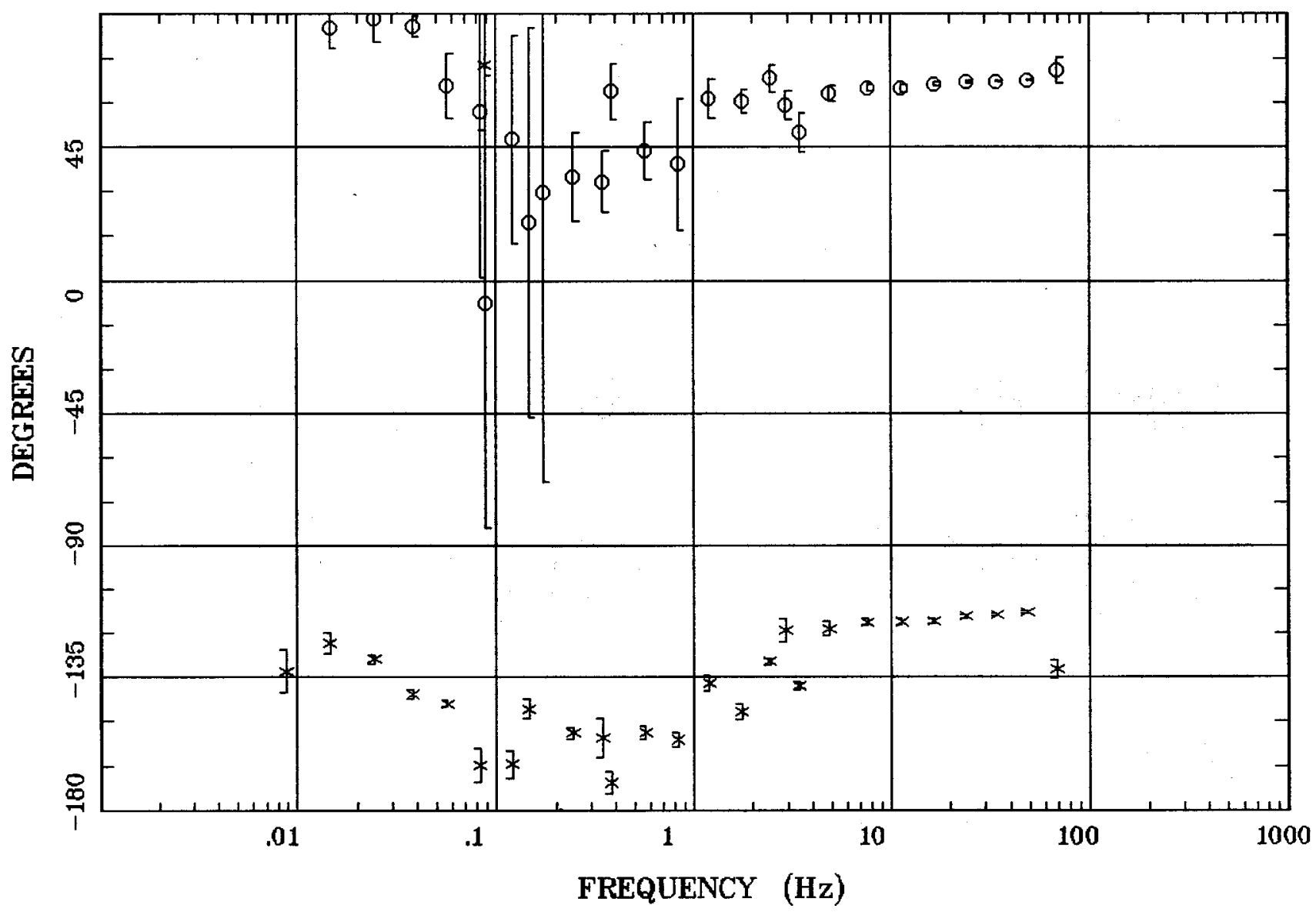

Client: Espanola Basin

Remote: none

Acquired: 11:1 Jul 14, 2004

Survey Co:USGS
Rotation:

Filename: cp31m68.avg

Channels: Ch1 Ch2 Ch3 Ch4 Ch5 Ch3 Ch4

Plotted: 14:01 Sep 01, 2004

< EMI - ElectroMagnetic Instruments > 


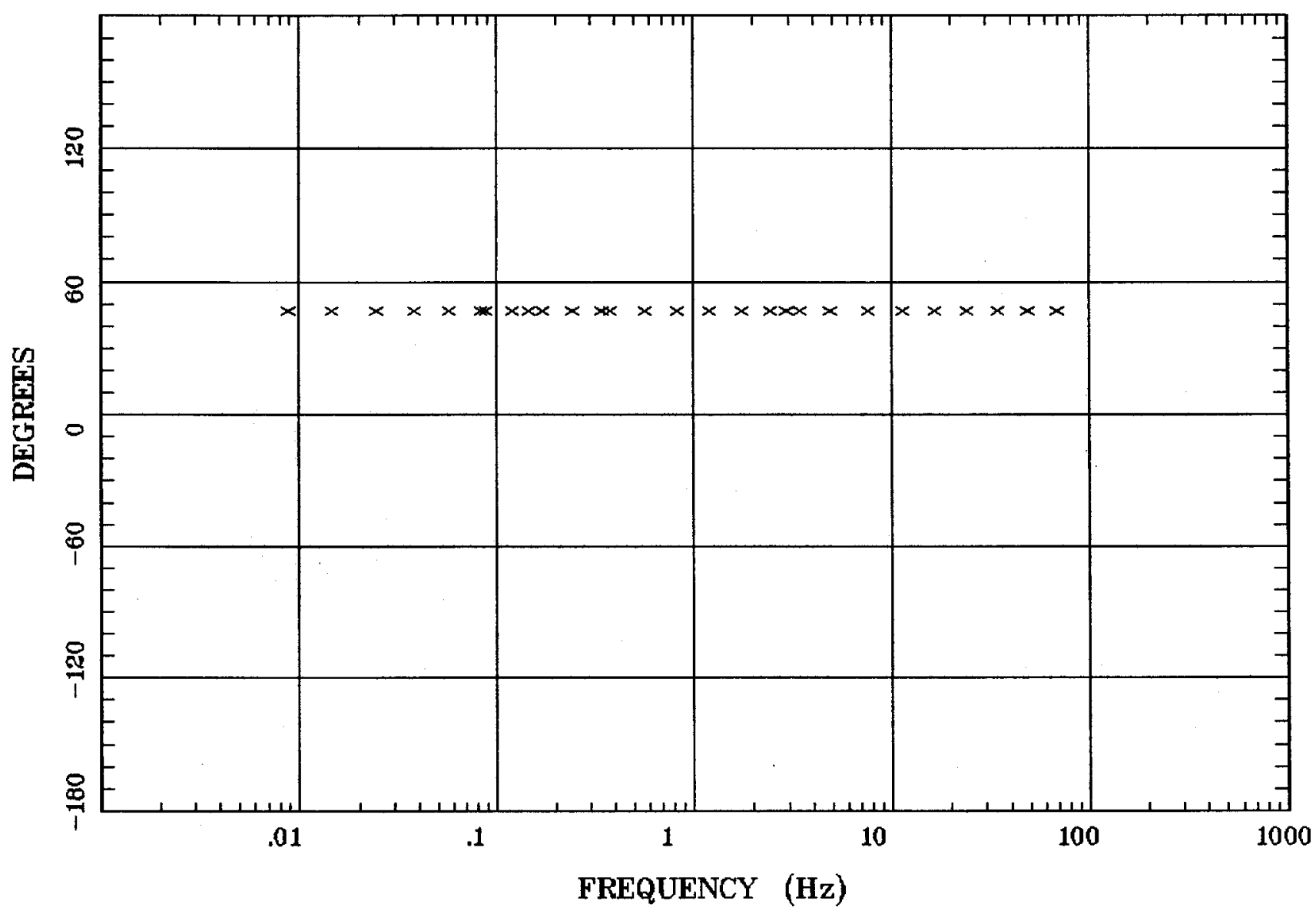

Client: Espanola Basin

Remote: none

Acquired: 11:1 Jul 14, 2004

Survey Co:USGS
Rotation:

Filename: cp31m68.avg

Channels: Ch1 Ch2 Ch3 Ch4 Ch5 Ch3 Ch4

Plotted: 14:01 Sep 01, 2004

< EMI - ElectroMagnetic Instruments 


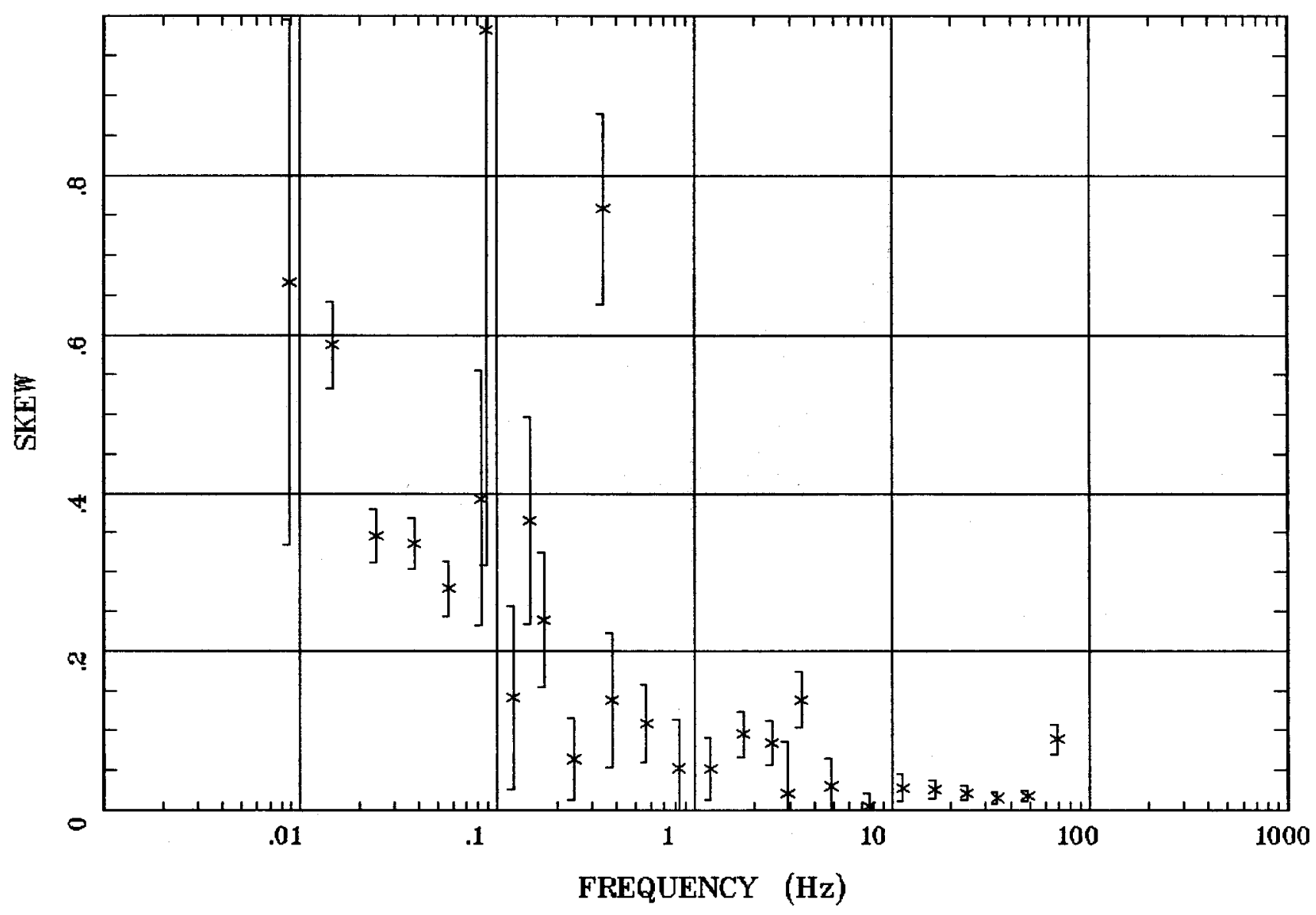

Client: Espanola Basin

Remote: none

Acquired: 11:1 Jul 14, 2004 Survey Co:USGS
Rotation:

Filename: cp31m68.avg

Channels: Ch1 Ch2 Ch3 Ch4 Ch5 Ch3 Ch4 Plotted: 14:01 Sep 01, 2004

< EMI - ElectroMagnetic Instruments 


\section{Station 31}

E MULT Coh.

Los Alamos, NM 100k

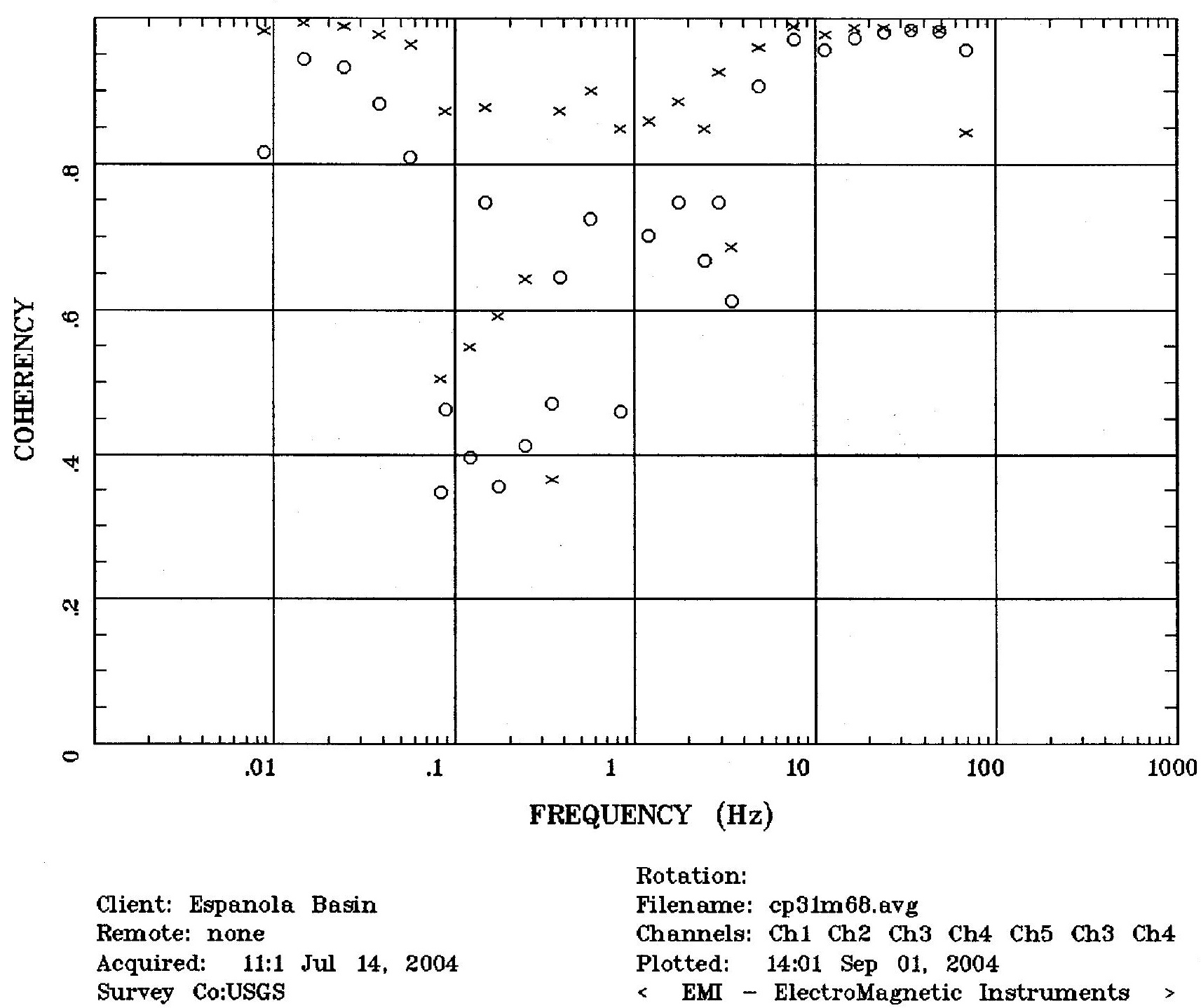




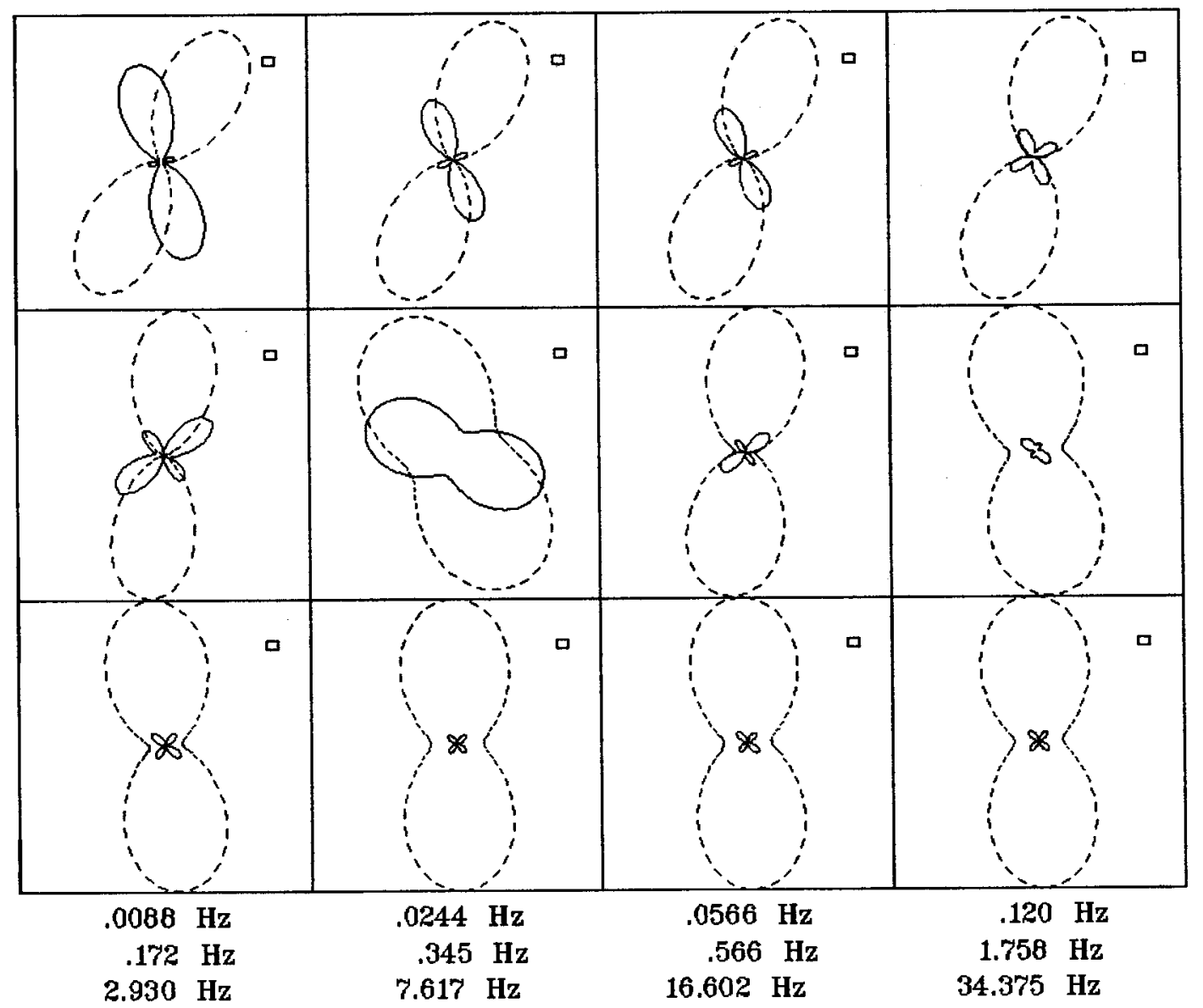

Client: Espanola Basin Remote: none

Acquired: 11:1 Jul 14, 2004 Survey Co:USGS
Rotation:

Filename: cp31m68.avg

Channels: Ch1 Ch2 Ch3 Ch4 Ch5 Ch3 Ch4 Plotted: 14:01 Sep 01, 2004

< EMI - ElectroMagnetic Instruments 


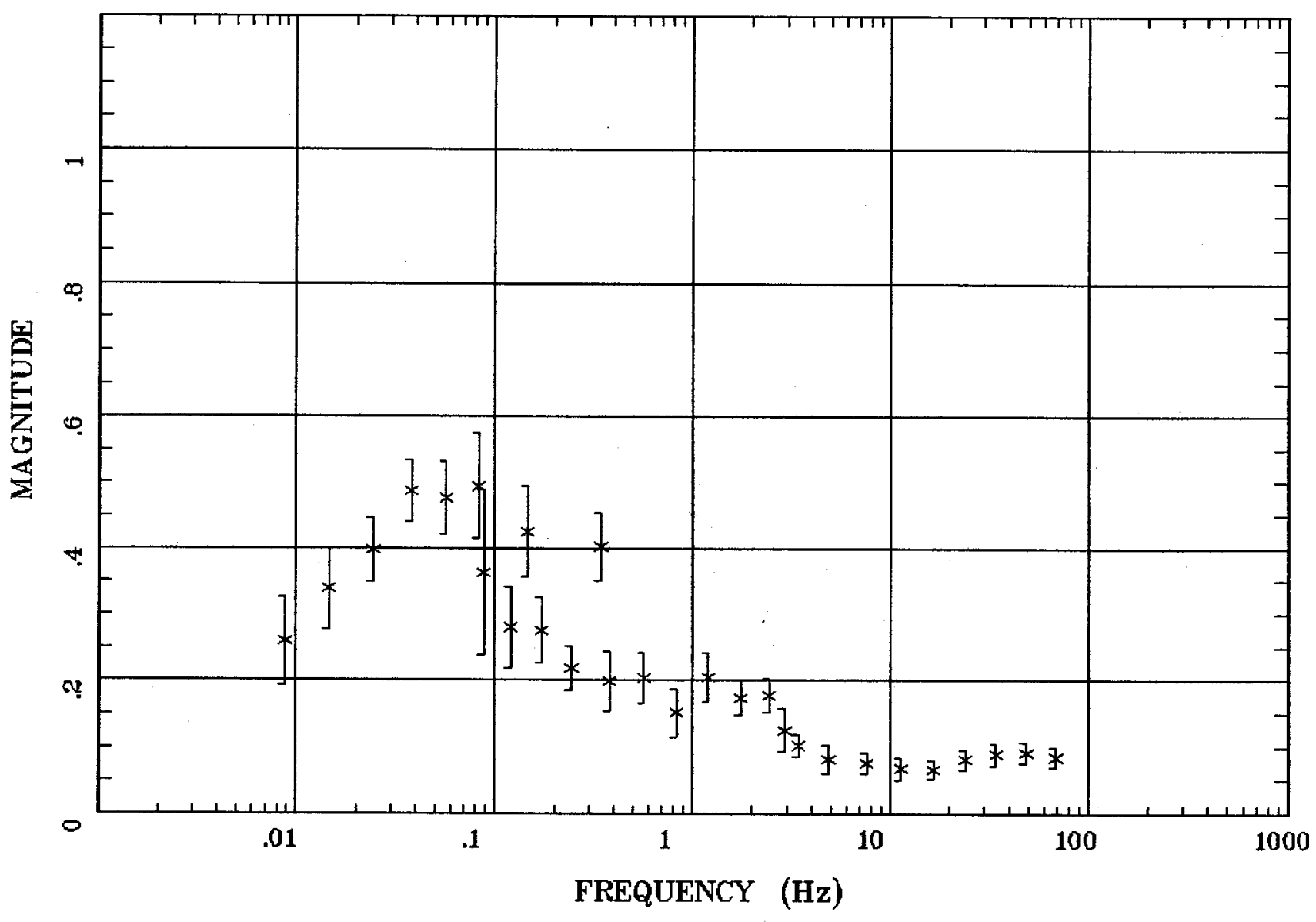

Client: Espanola Basin

Remote: none

Acquired: 11:1 Jul 14, 2004

Survey Co:USGS
Rotation:

Filename: cp31m68.avg

Channels: Ch1 Ch2 Ch3 Ch4 Ch5 Ch3 Ch4

Plotted: 14:01 Sep 01, 2004

$<$ EMI - ElectroMagnetic Instruments 


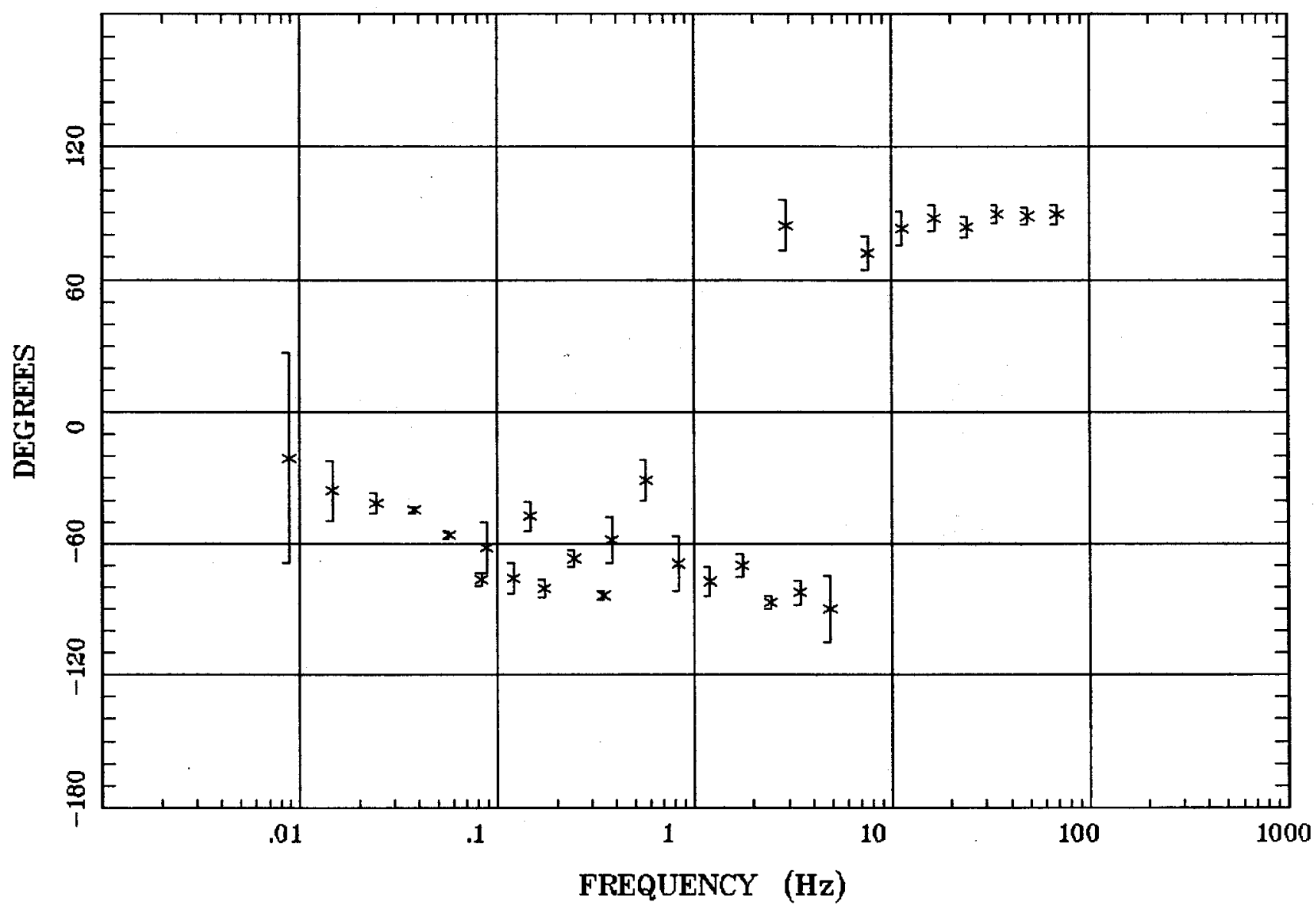

Client: Espanola Basin Remote: none

Acquired: 11:1 Jul 14, 2004 Survey Co:USGS
Rotation:

Filename: cp31m68.avg

Channels: Ch1 Ch2 Ch3 Ch4 Ch5 Ch3 Ch4 Plotted: 14:01 Sep 01, 2004

< EMI - ElectroMagnetic Instruments 
HzHx.x Coh HzHy.o

Los Alamos, NM 100k

Station 31

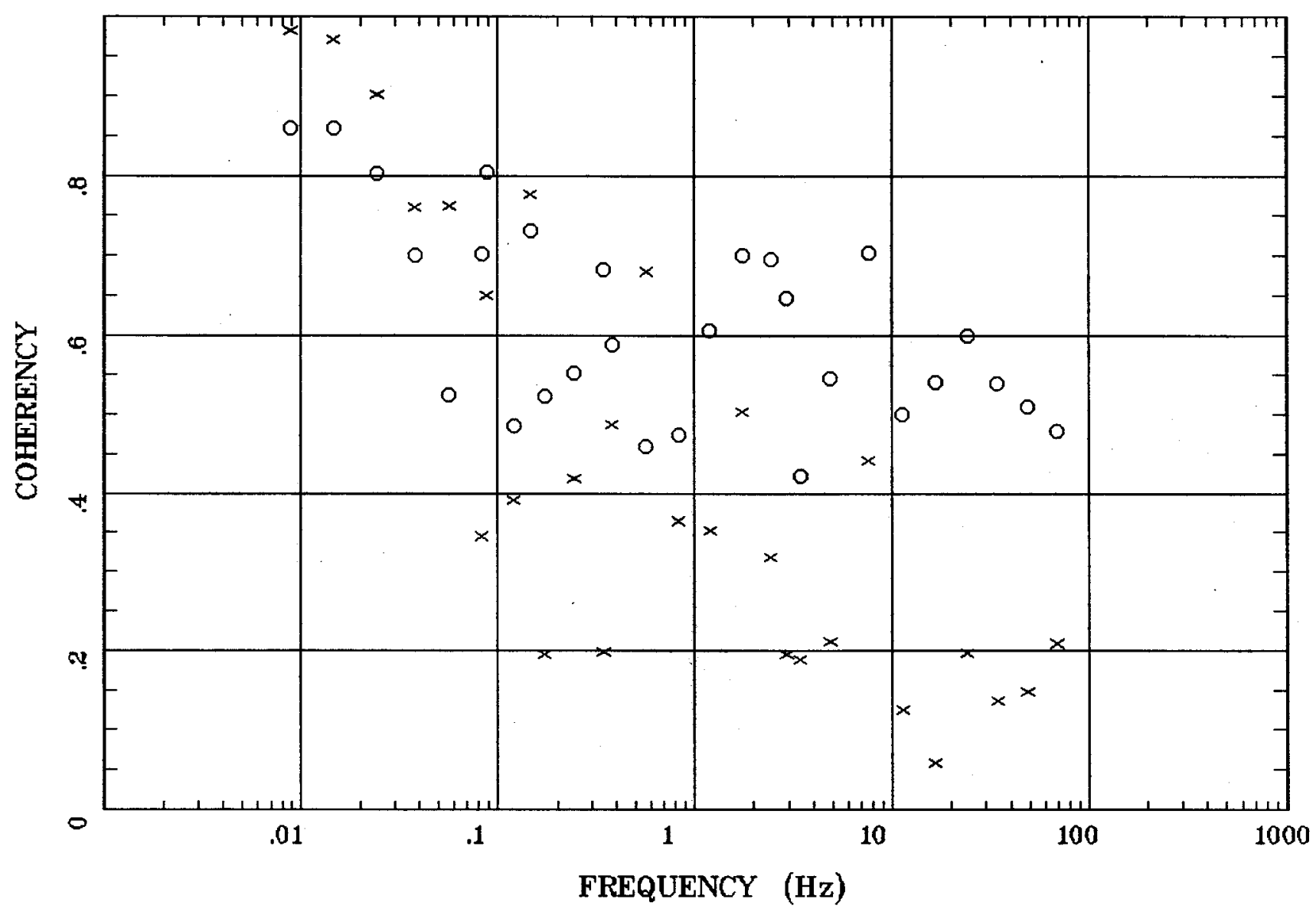

Client: Espanola Basin Remote: none

Acquired: 11:1 Jul 14, 2004 Survey Co:USGS
Rotation:

Filename: cp31m68.avg

Channels: Ch1 Ch2 Ch3 Ch4 Ch5 Ch3 Ch4 Plotted: 14:01 Sep 01, 2004

< EMI - ElectroMagnetic Instruments > 
APPARENT RESISTIVITY

Los Alomose NM 100k

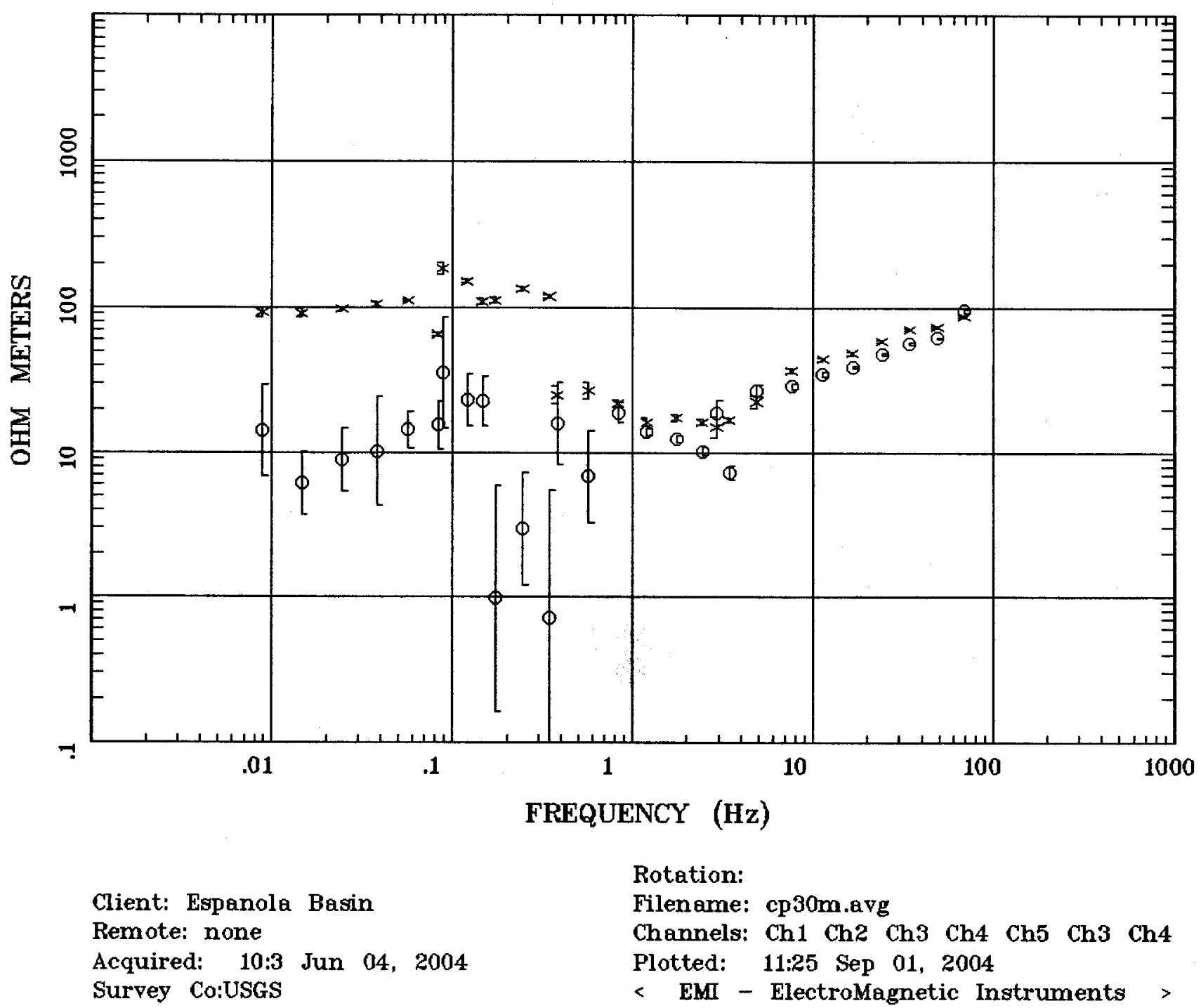




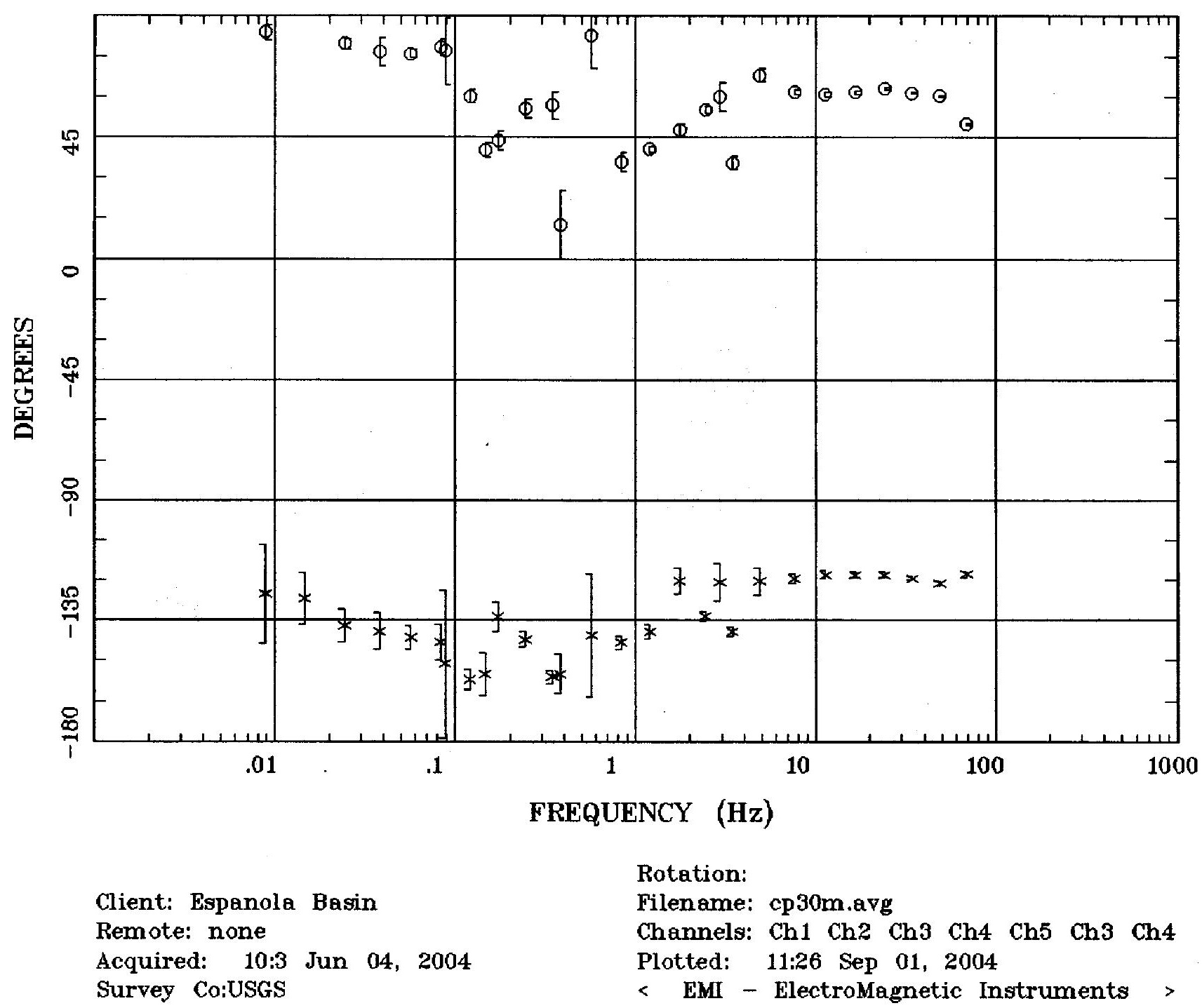


ROTATION ANGLE

Los Alomose NM 100k

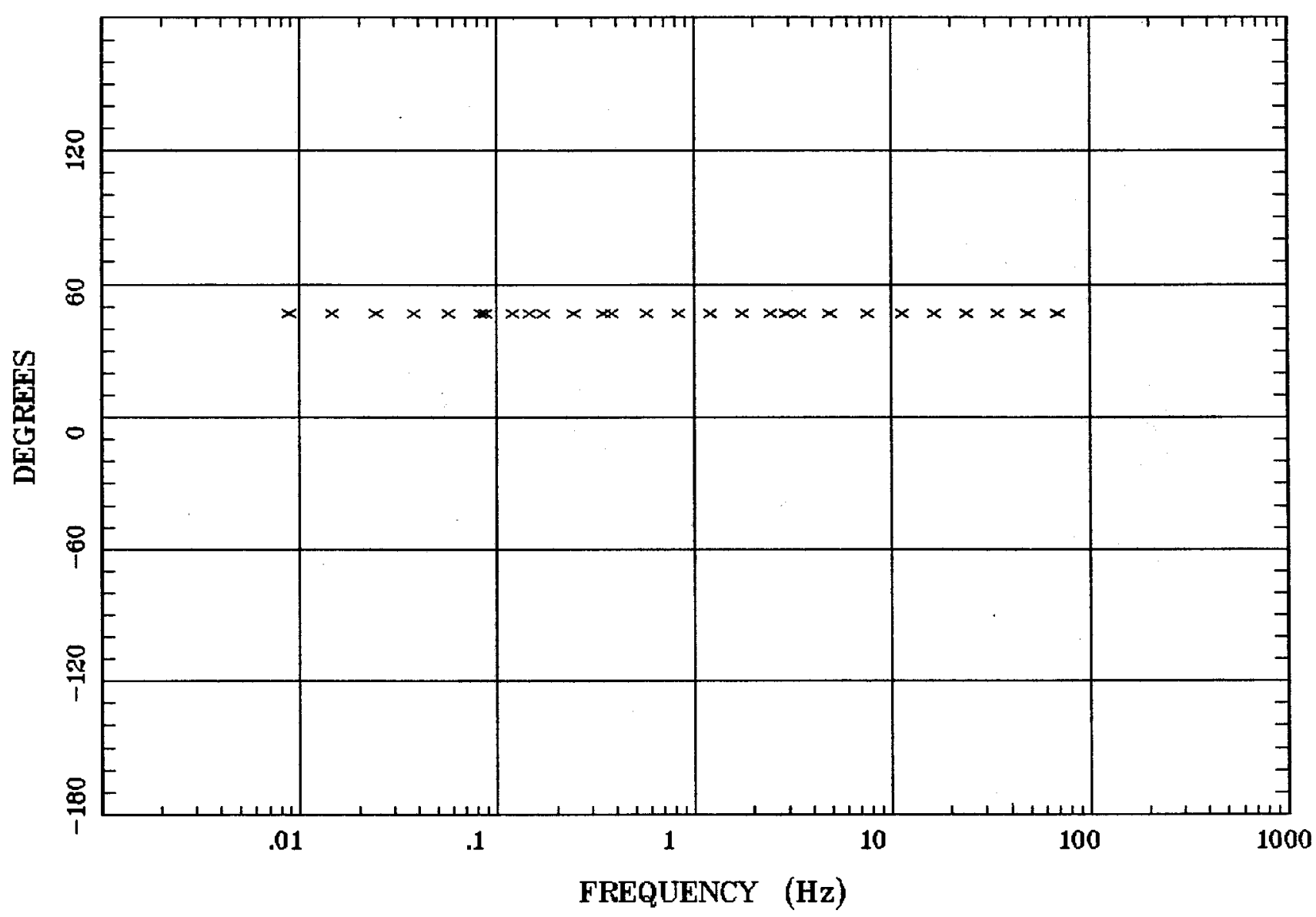

Client: Espanola Basin

Remote: none

Acquired: 10:3 Jun 04, 2004 Survey Co:USGS
Rotation:

Filename: cp30m.avg

Channels: Ch1 Ch2 Ch3 Ch4 Ch5 Ch3 Ch4 Plotted: 11:26 Sep 01, 2004

$<$ EMI - ElectroMagnetic Instruments 


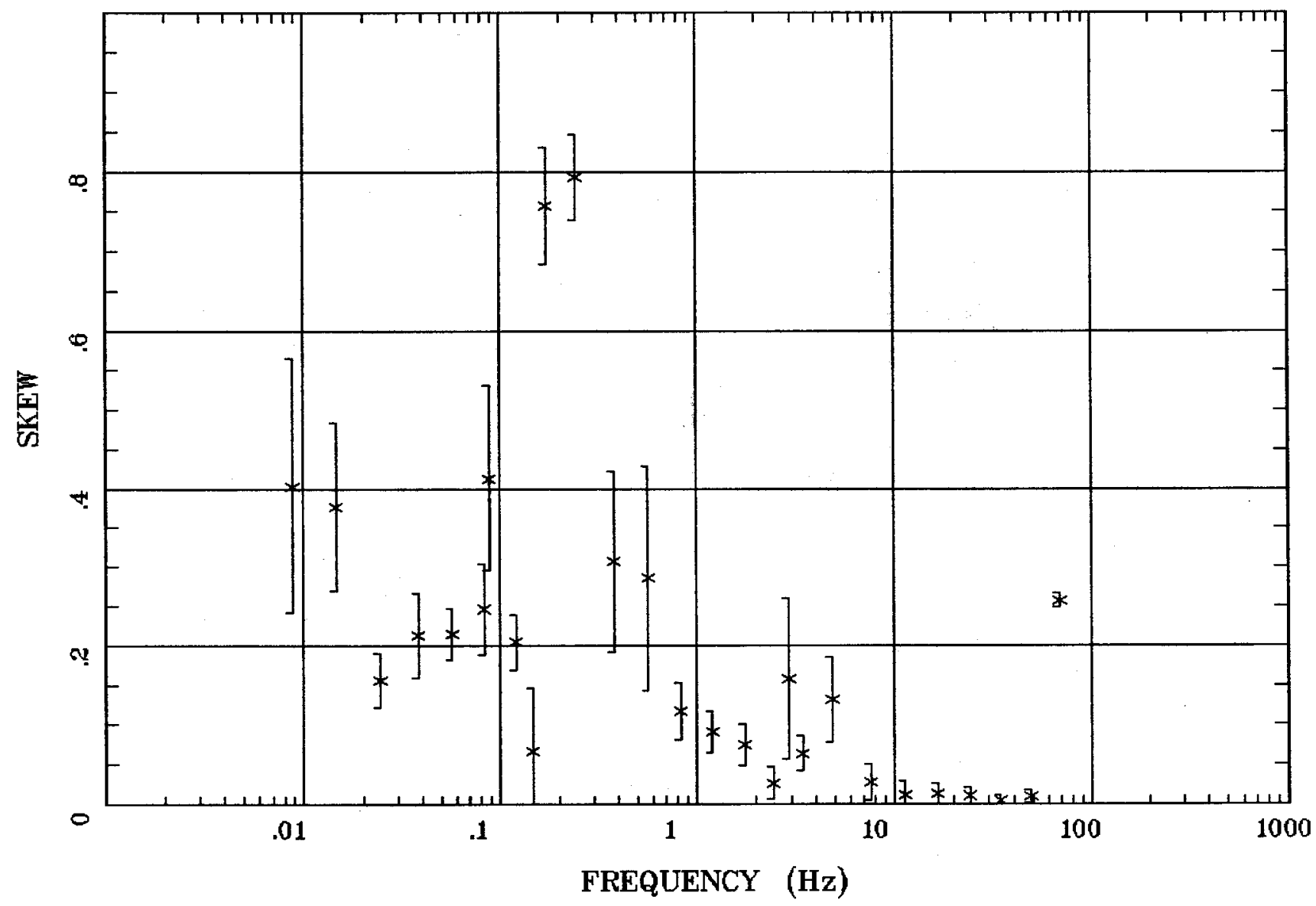

Client: Espanola Basin Remote: none Acquired: 10:3 Jun 04, 2004 Survey Co:USGS
Rotation:

Filename: cp $30 \mathrm{~m}$.avg

Channels: Ch1 Ch2 Ch3 Ch4 Ch5 Ch3 Ch4 Plotted: 11:26 Sep 01, 2004

< EMI - ElectroMagnetic Instruments > 

E MULT Coh.
Los Alomose NM 100k

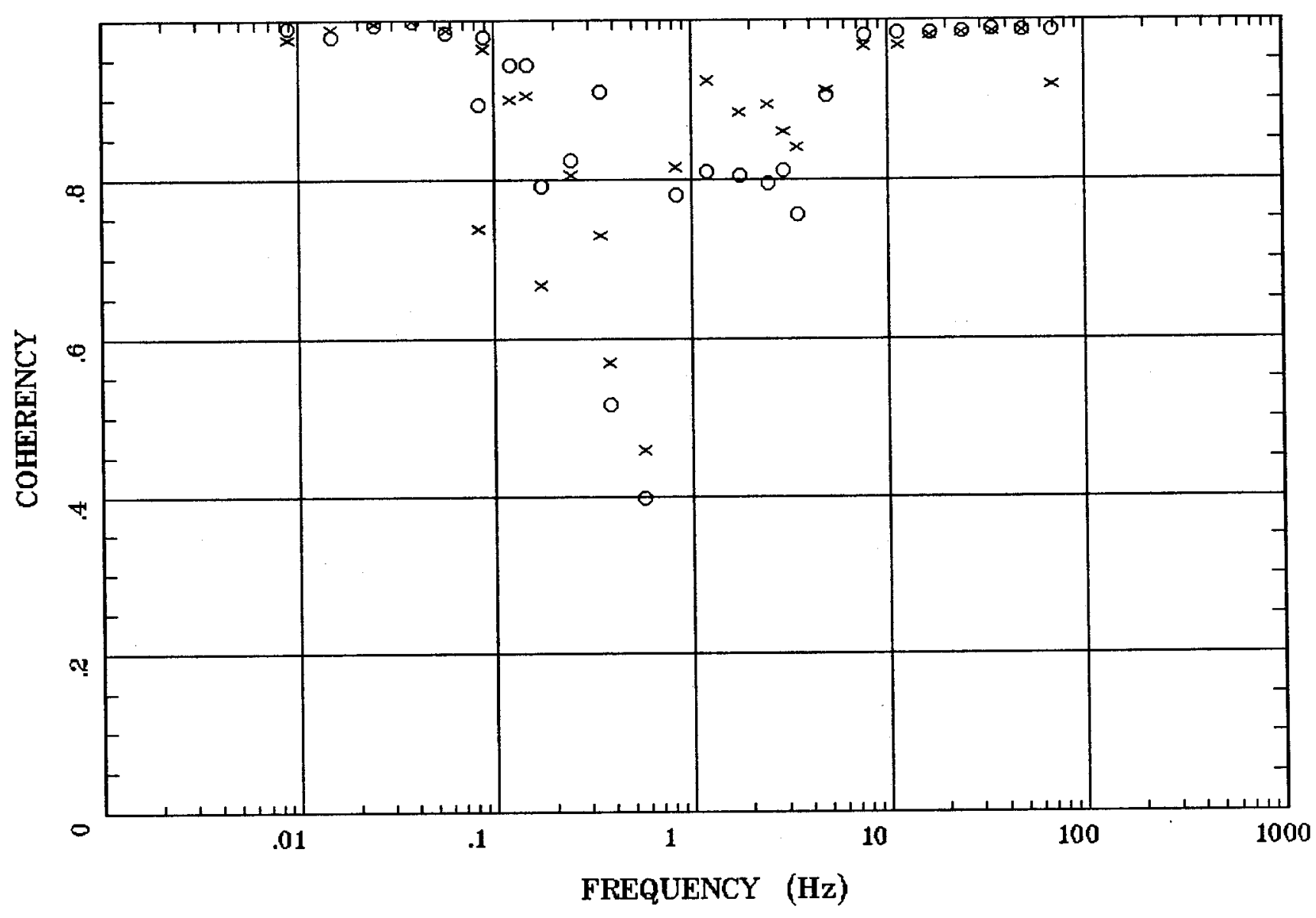

Client: Espanola Basin

Remote: none

Acquired: 10:3 Jun 04, 2004 Survey Co:USGS
Rotation:

Filename: cp30m.avg

Channels: Ch1 Ch2 Ch3 Ch4 Ch5 Ch3 Ch4 Plotted: 11:26 Sep 01, 2004

< EMI - ElectroMagnetic Instruments 


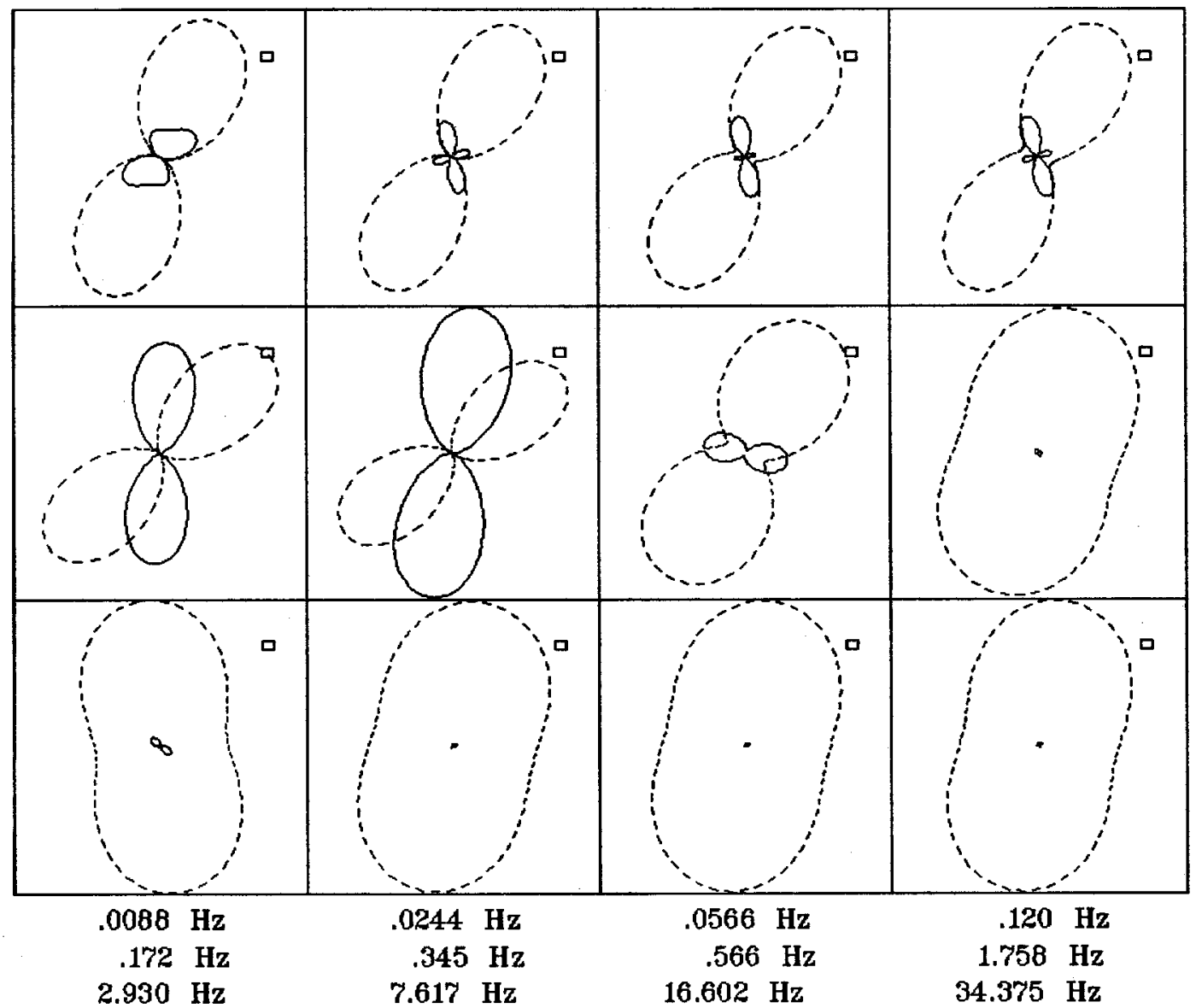

Client: Espanola Basin

Remote: none

Acquired: 10:3 Jun 04, 2004 Survey Co:USGS
Rotation:

Filename: cp $30 \mathrm{~m} . \mathrm{avg}$

Channels: Ch1 Ch2 Ch3 Ch4 Ch5 Ch3 Ch4 Platted: 11:26 Sep 01, 2004

< EMI - ElectroMagnetic Instruments 


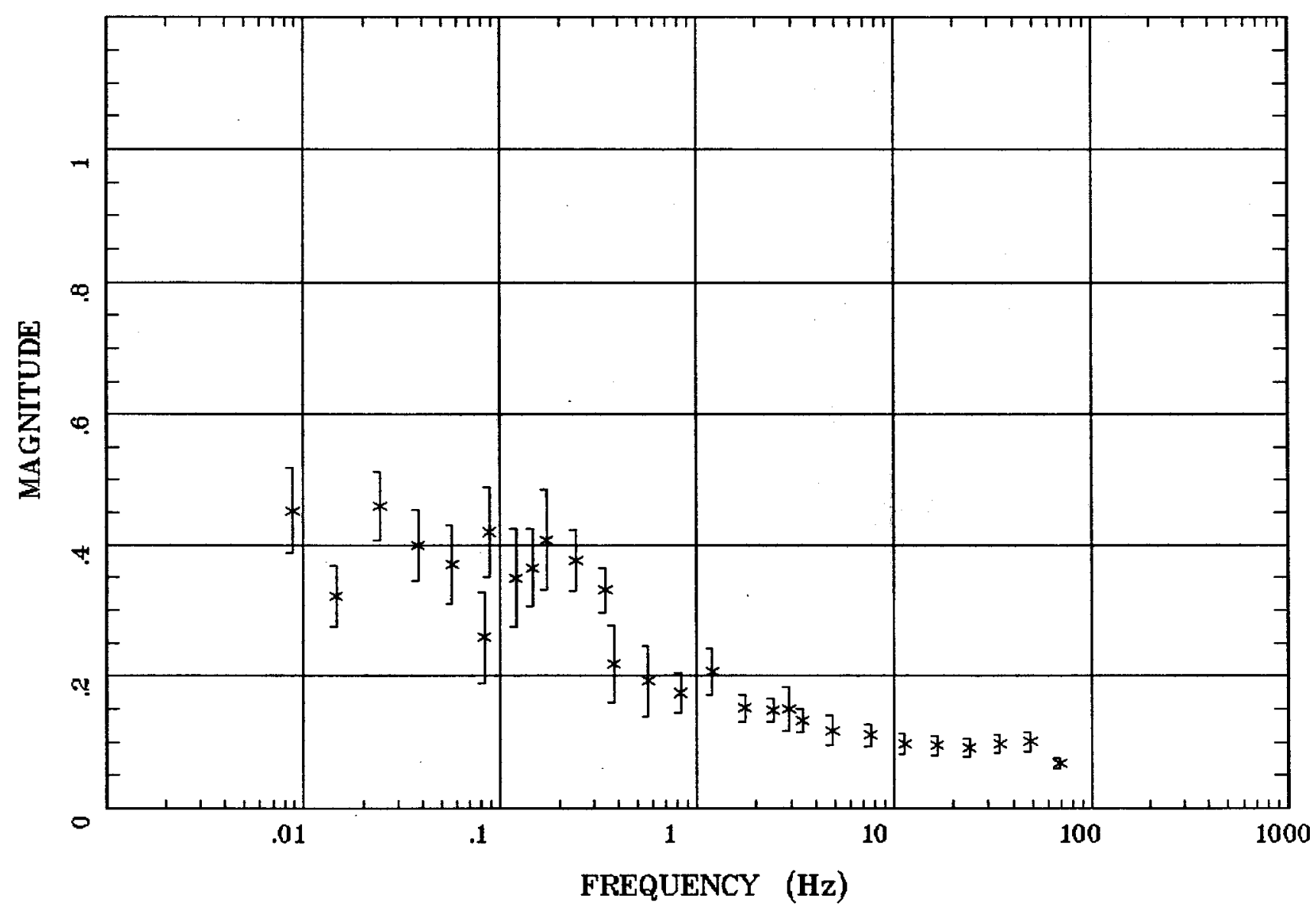

Client: Espanala Basin Remote: none Acquired: 10:3 Jun 04, 2004 Survey Co:USGS
Rotation:

Filename: cp30m.avg

Channels: Ch1 Ch2 Ch3 Ch4 Ch5 Ch3 Ch4 Plotted: 11:26 Sep 01, 2004

< EMI - ElectroMagnetic Instruments 
Station 30

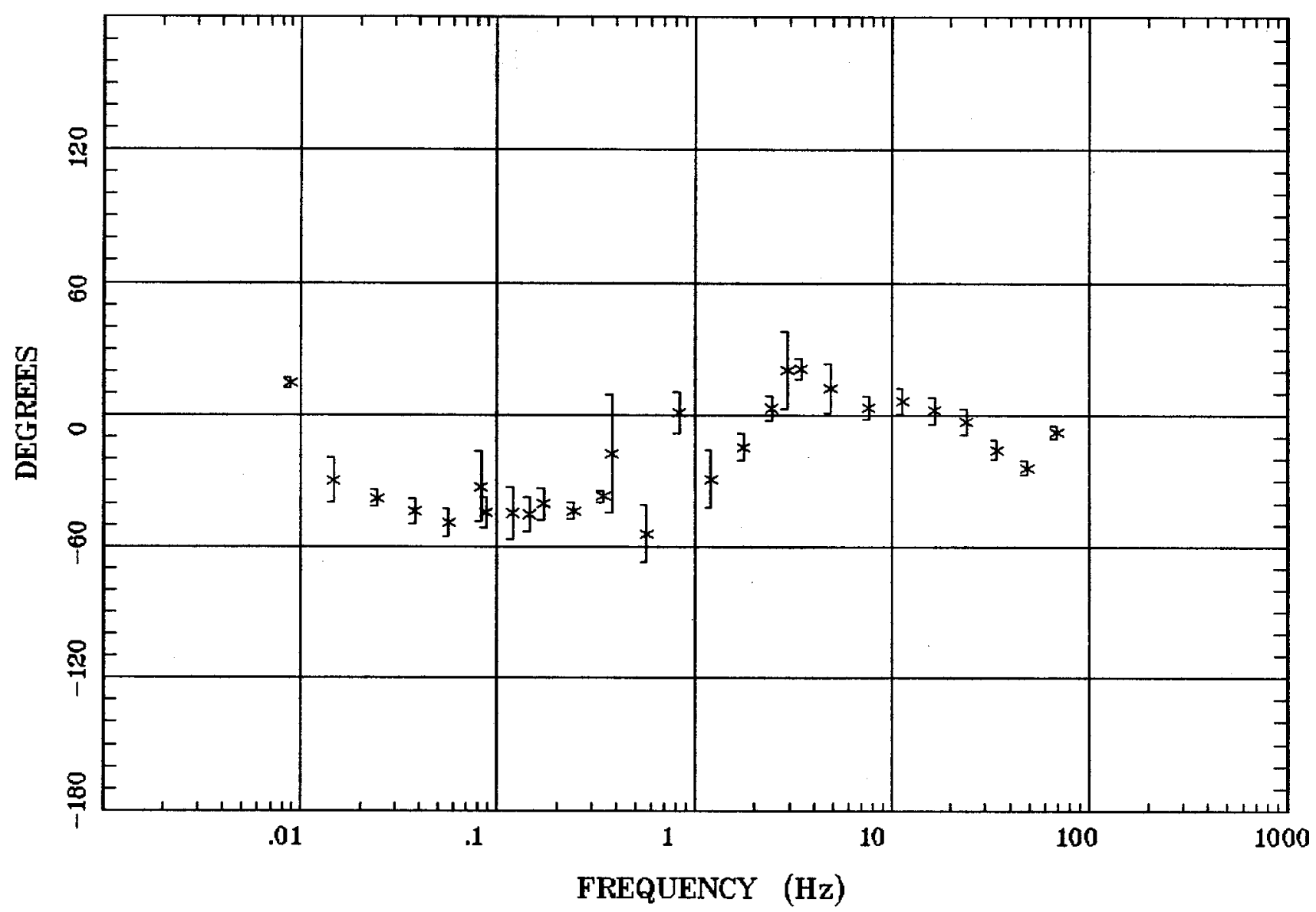

Client: Espanola Basin Remote: none Acquired: 10:3 Jun 04, 2004 Survey Co:USGS

\section{Rotation:}

Filen ame: cp30m.avg

Channels: Ch1 Ch2 Ch3 Ch4 Ch5 Ch3 Ch4 Platted: 11:26 Sep 01, 2004

< EMI - ElectroMagnetic Instruments > 
HzHx.x Coh HzHy.o

Los Alomose NM 100k

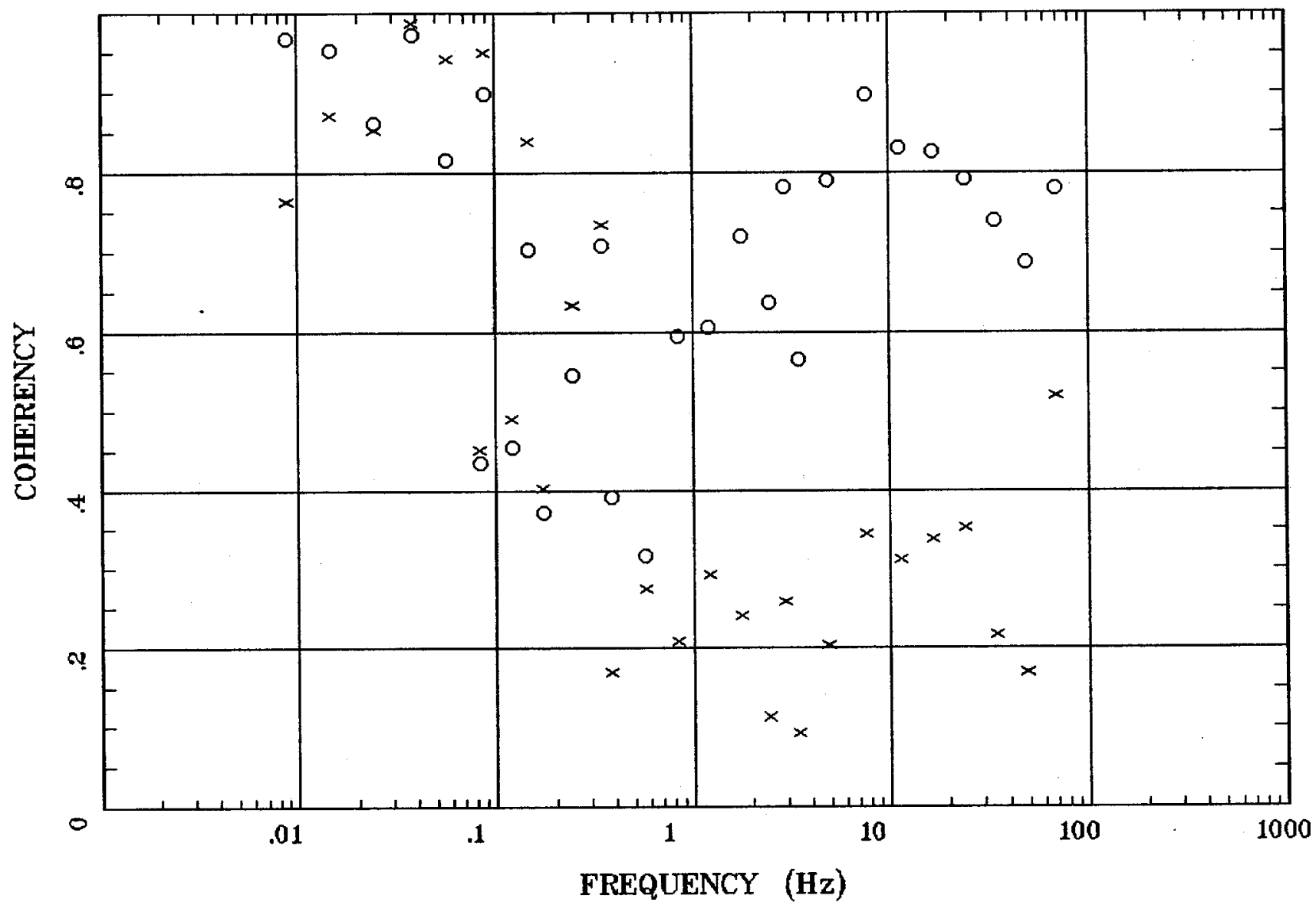

Client: Espanola Basin

Remote: none

Acquired: 10:3 Jun 04, 2004

Survey Co:USGS
Rotation:

Filename: cp30m.avg

Channels: Ch1 Ch2 Ch3 Ch4 Ch5 Ch3 Ch4

Platted: 11:26 Sep 01, 2004

< EMI - ElectroMagnetic Instruments > 
Station 36

APPARENT RESISTIVITY

Los Alamos, NM 100k

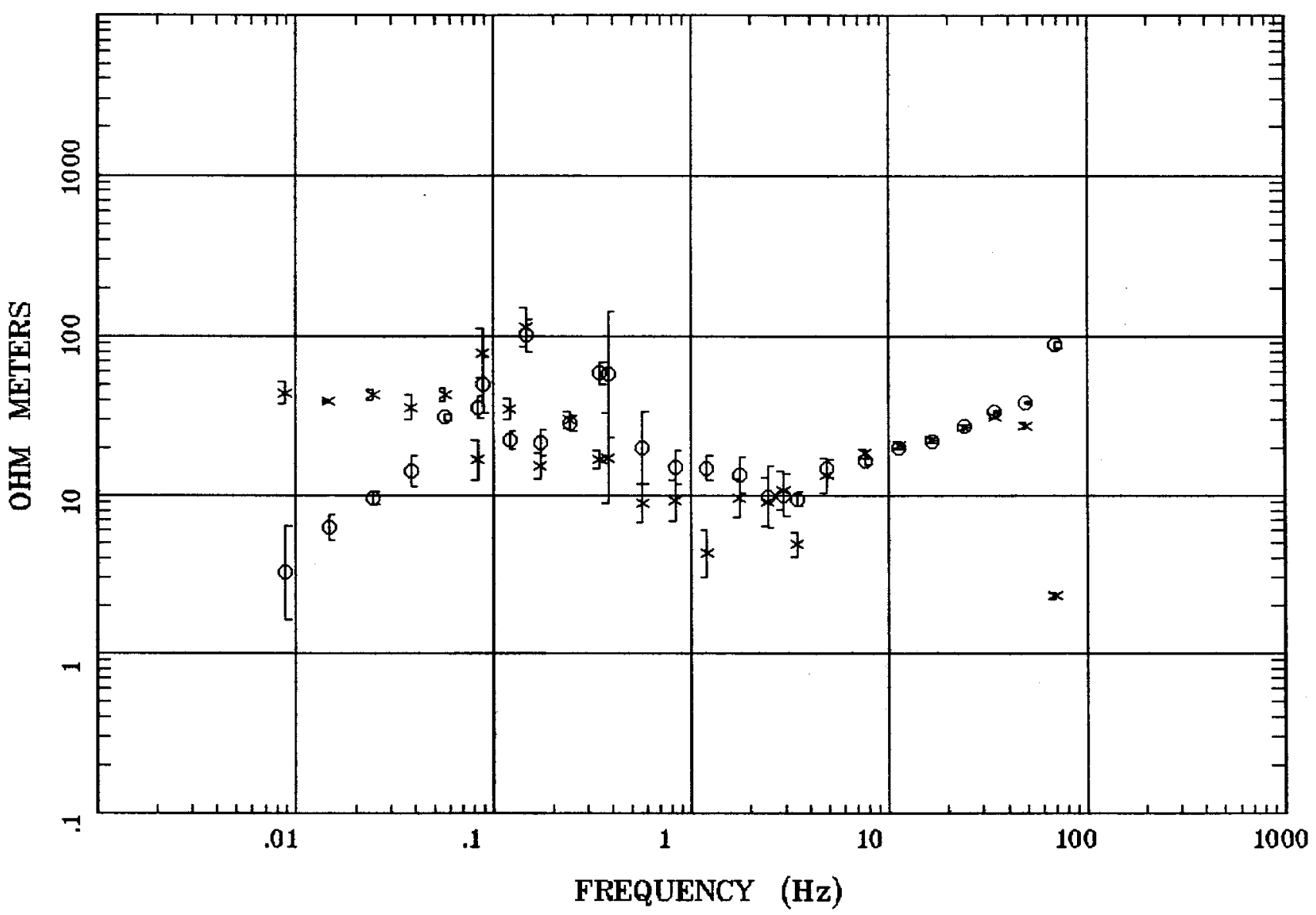

Client: Espanola Basin

Remote: none

Acquired: 10:1 Jun 07, 2004

Survey Co:USGS

\section{Rotation:}

Filename: cp36m5.avg

Channels: Ch1 Ch2 Ch3 Ch4 Ch5 Ch3 Ch4

Plotted: 15:11 Aug 04, 2004

$<$ EMI - ElectroMagnetic Instruments 
Los Alamos, NM 100k

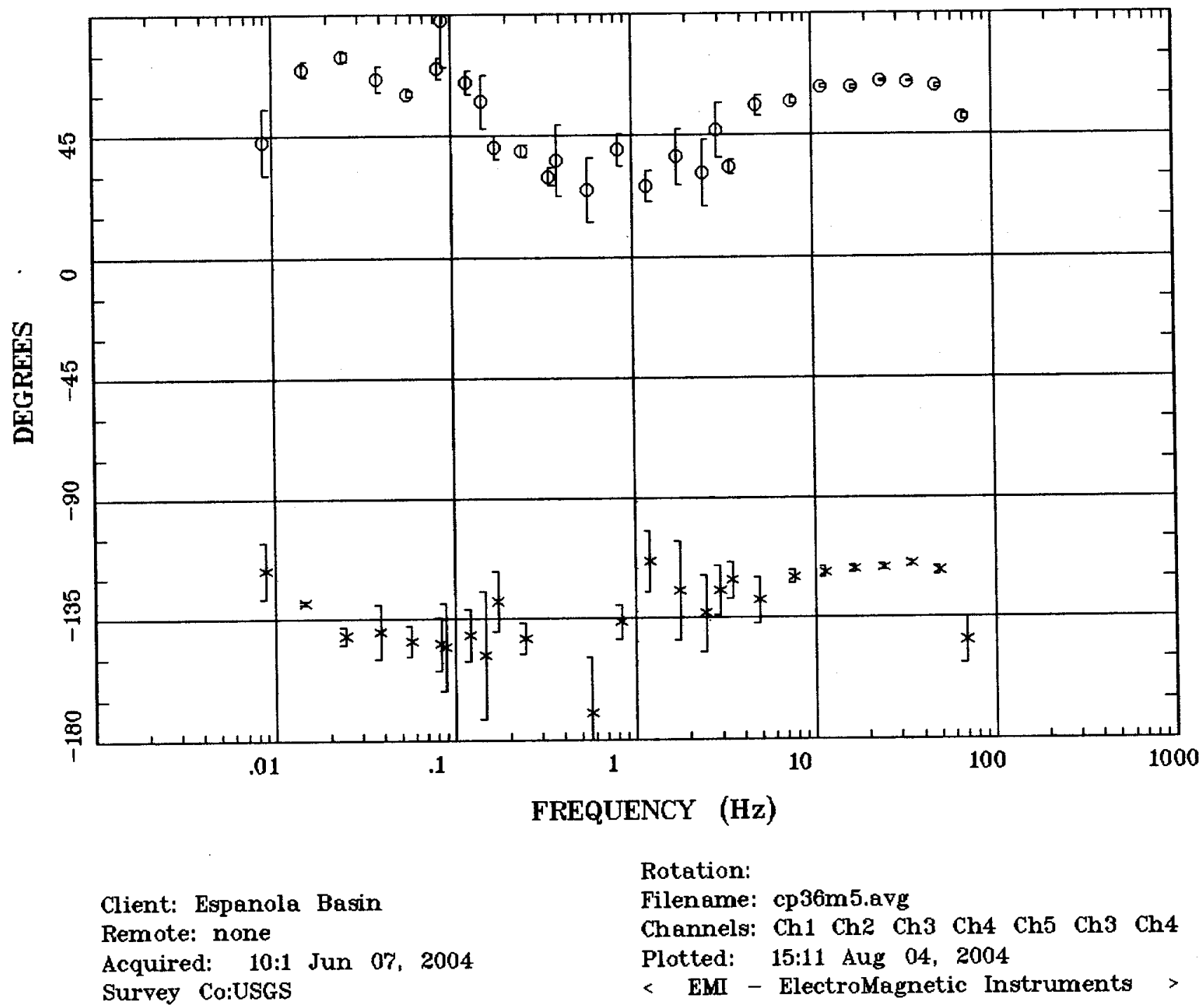




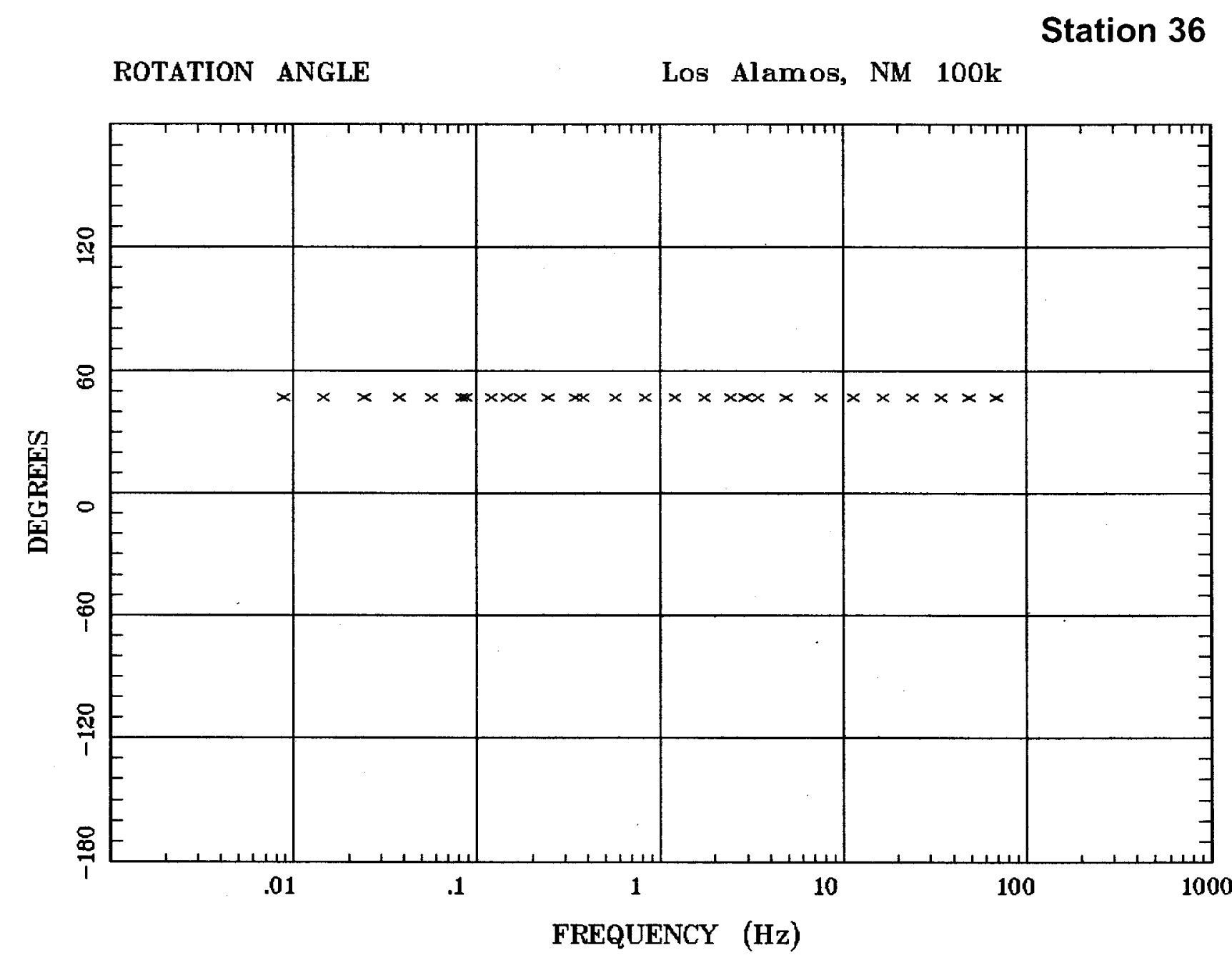

Client: Espanola Basin

Remote: none

Acquired: 10:1 Jun 07, 2004

Survey Co:USGS
Rotation:

Filename: cp36m5.avg

Channels: Ch1 Ch2 Ch3 Ch4 Ch5 Ch3 Ch4

Plotted: 15:11 Aug 04, 2004

< EMI - ElectroMagnetic Instruments > 


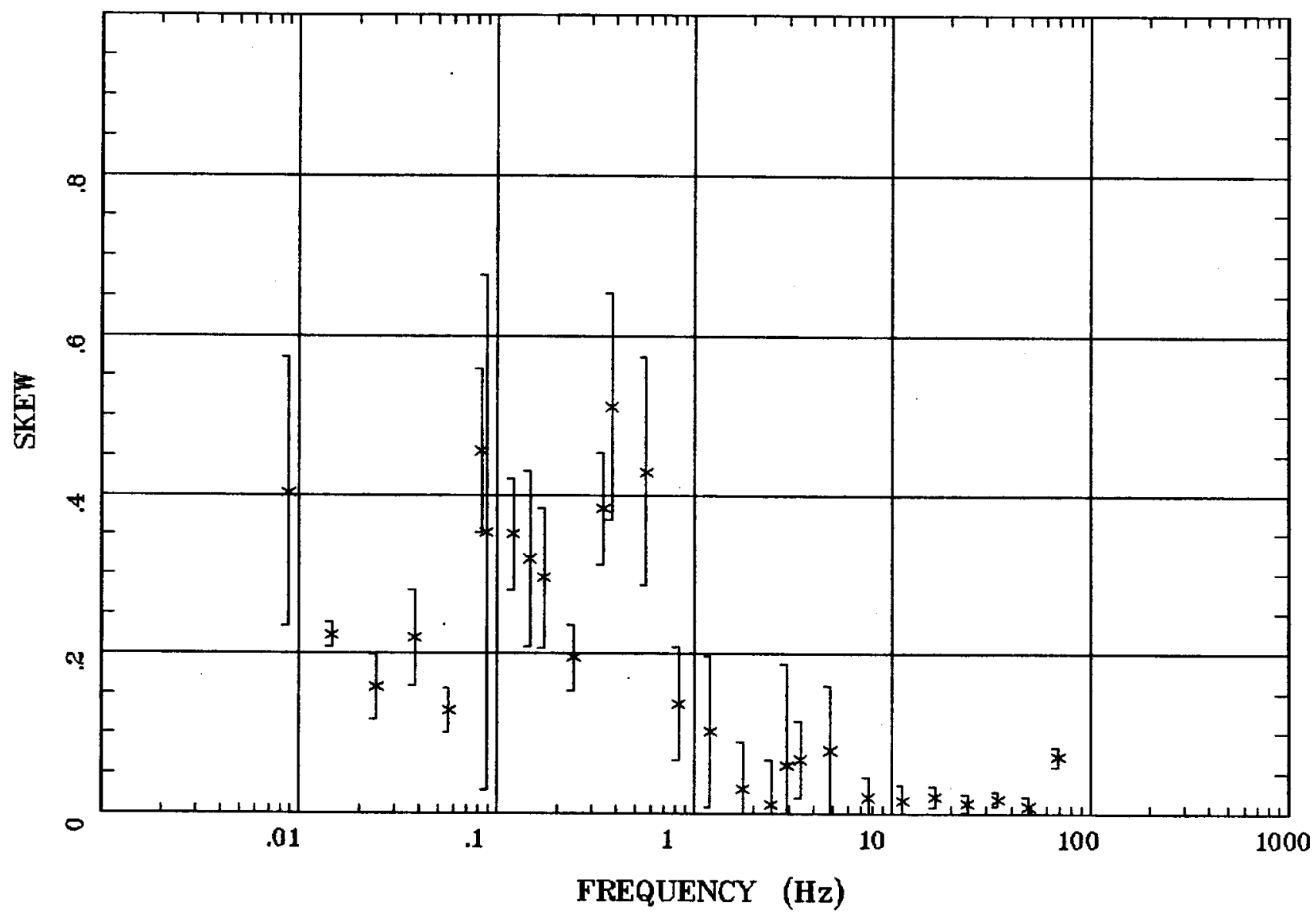

Client: Espanola Basin

Remote: none

Acquired: 10:1 Jun 07, 2004

Survey Co:USGS
Rotation:

Filename: cp36m5.avg

Channels: Ch1 Ch2 Ch3 Ch4 Ch5 Ch3 Ch4

Plotted: 15:11 Aug 04, 2004

$<$ EMI - ElectroMagnetic Instruments 
Station 36

E MULT Coh.

Los Alamos, NM 100k

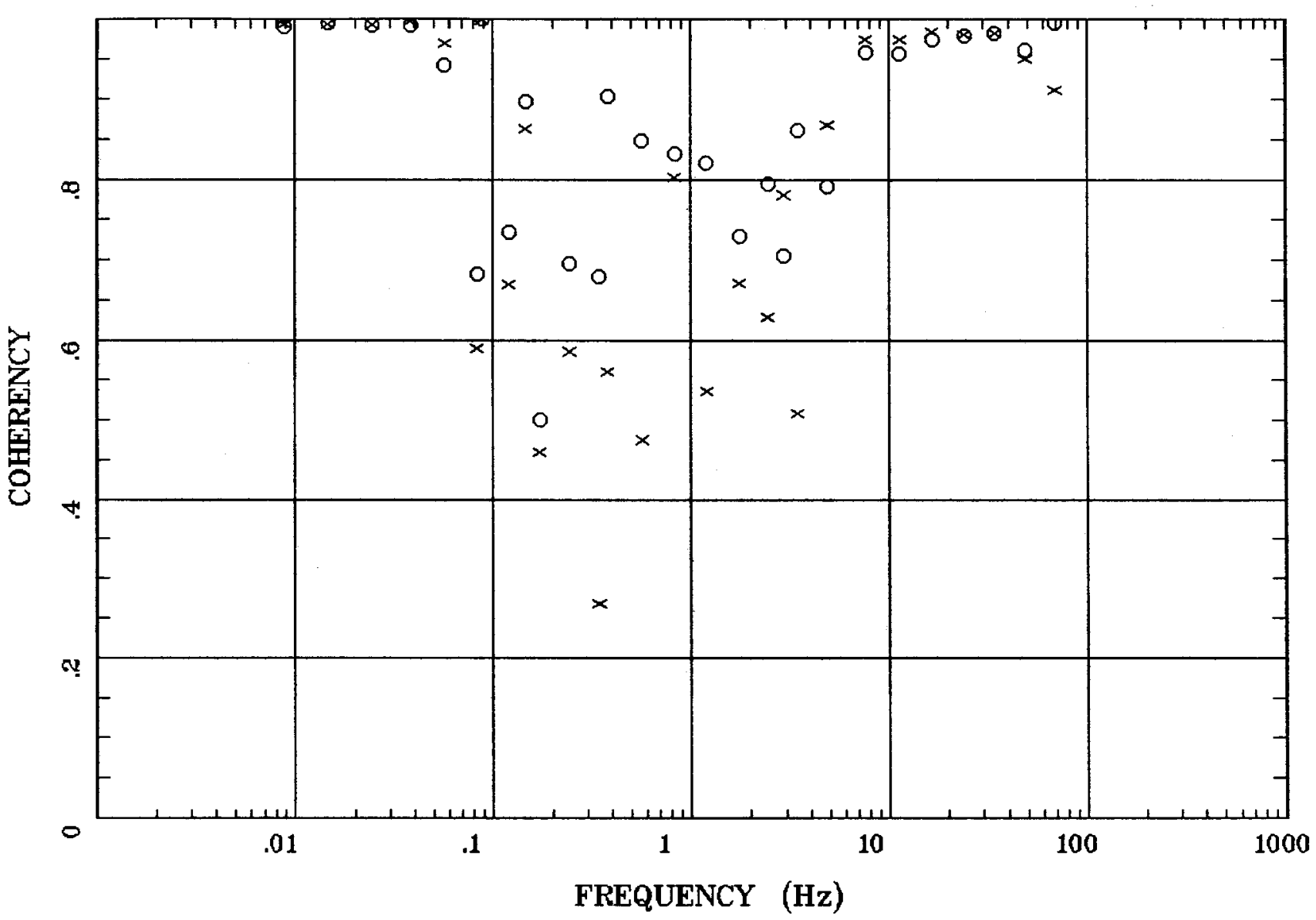

Client: Espanala Basin

Remote: none

Acquired: 10:1 Jun 07, 2004

Survey Co:USGS
Rotation:

Filename: cp36m5.avg

Channels: Ch1 Ch2 Ch3 Ch4 Ch5 Ch3 Ch4

Plotted: 15:11 Aug 04, 2004

$<$ EMI - ElectroMagnetic Instruments 
Station 36

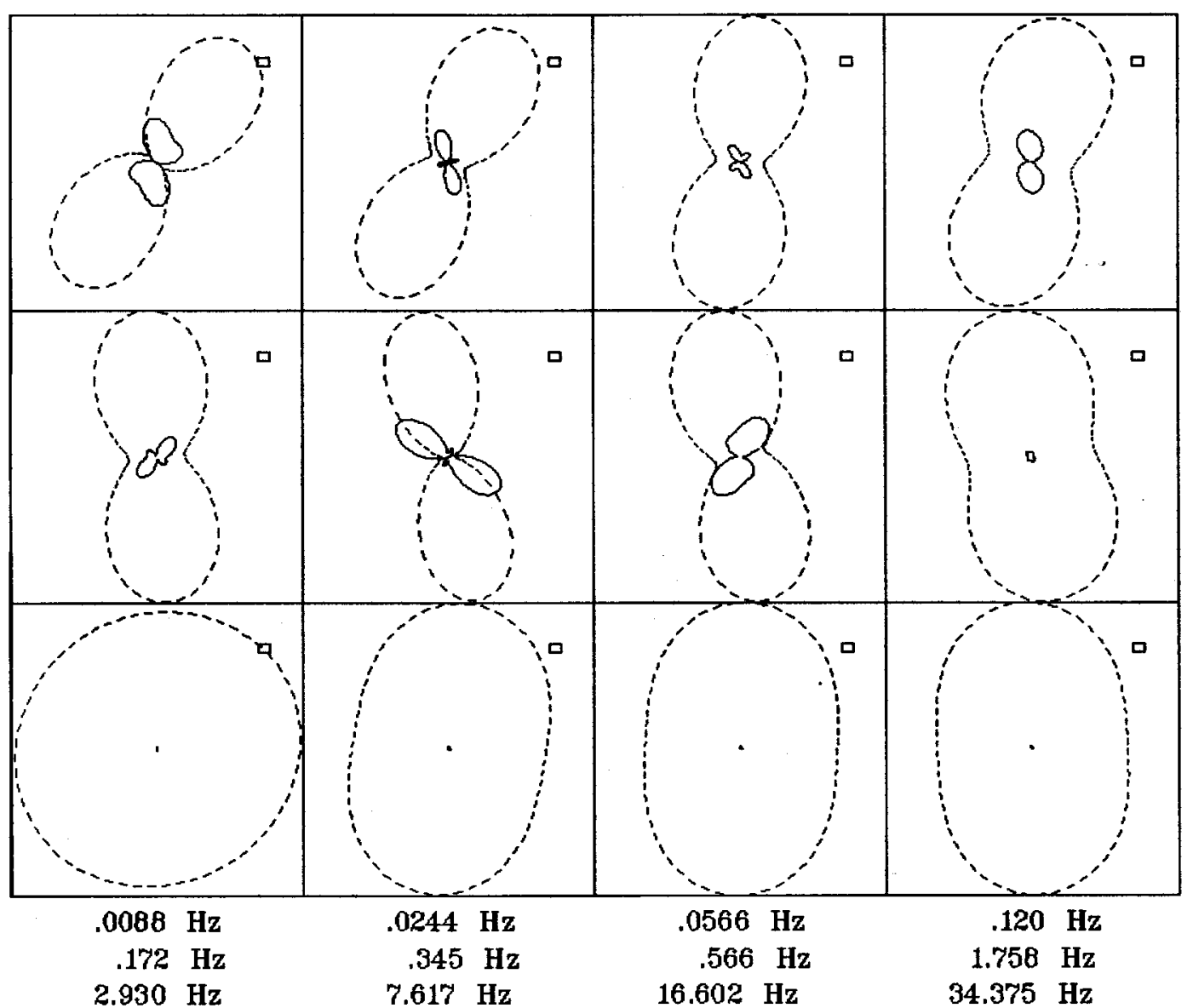

Client: Espanola Basin Remote: none Acquired: 10:1 Jun 07, 2004 Survey Co:USGS
Rotation:

Filename: cp36m5.avg Channels: Ch1 Ch2 Ch3 Ch4 Ch5 Ch3 Ch4 Plotted: 15:11 Aug 04, 2004

$<$ EMI - ElectroMagnetic Instruments 


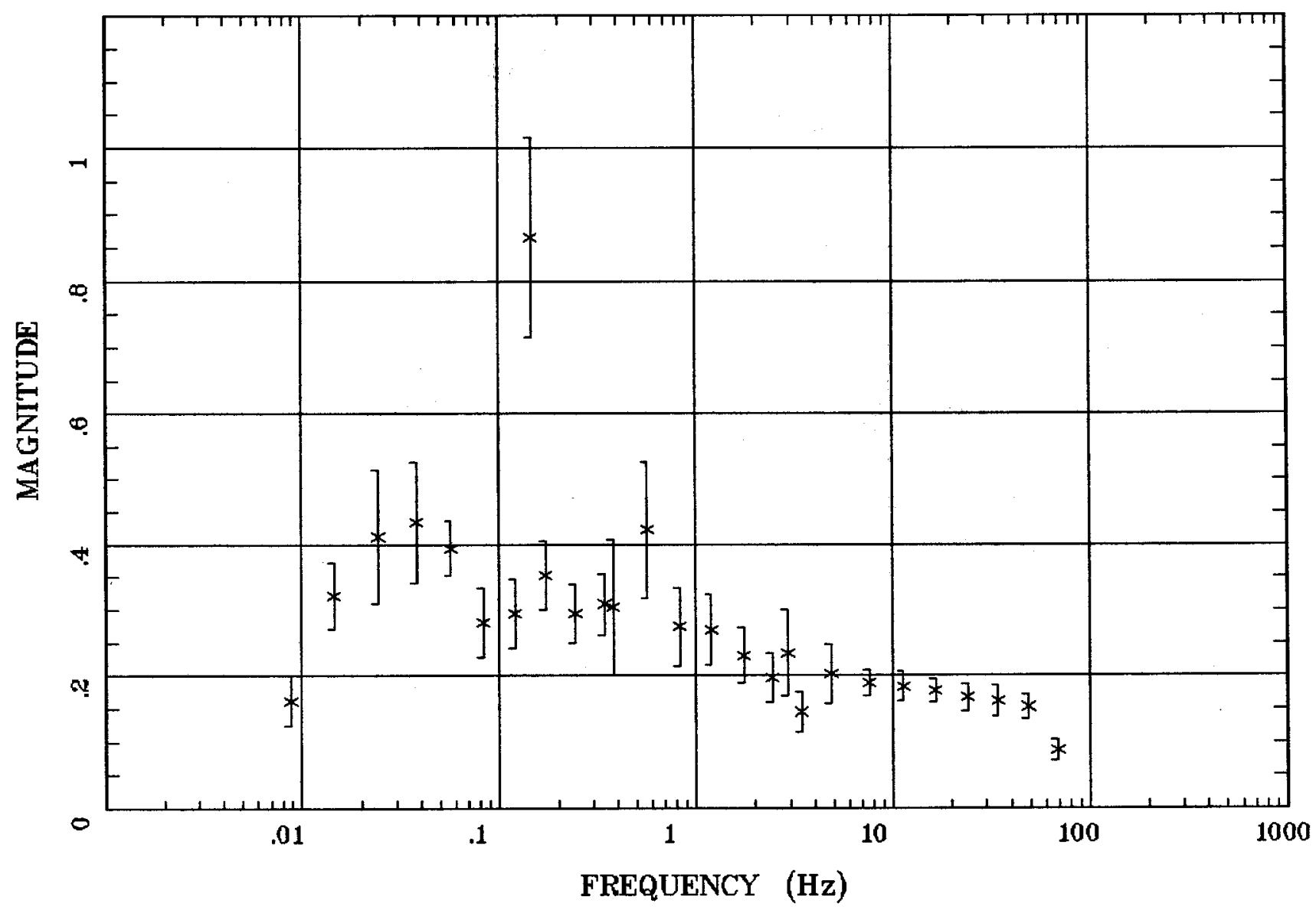

Client: Espanola Basin Remote: none Acquired: 10:1 Jun 07, 2004 Survey Co:USGS
Rotation:

Filename: cp36m5.avg

Channels: Ch1 Ch2 Ch3 Ch4 Ch5 Ch3 Ch4 Platted: 15:11 Aug 04, 2004

< EMI - ElectroMagnetic Instruments 


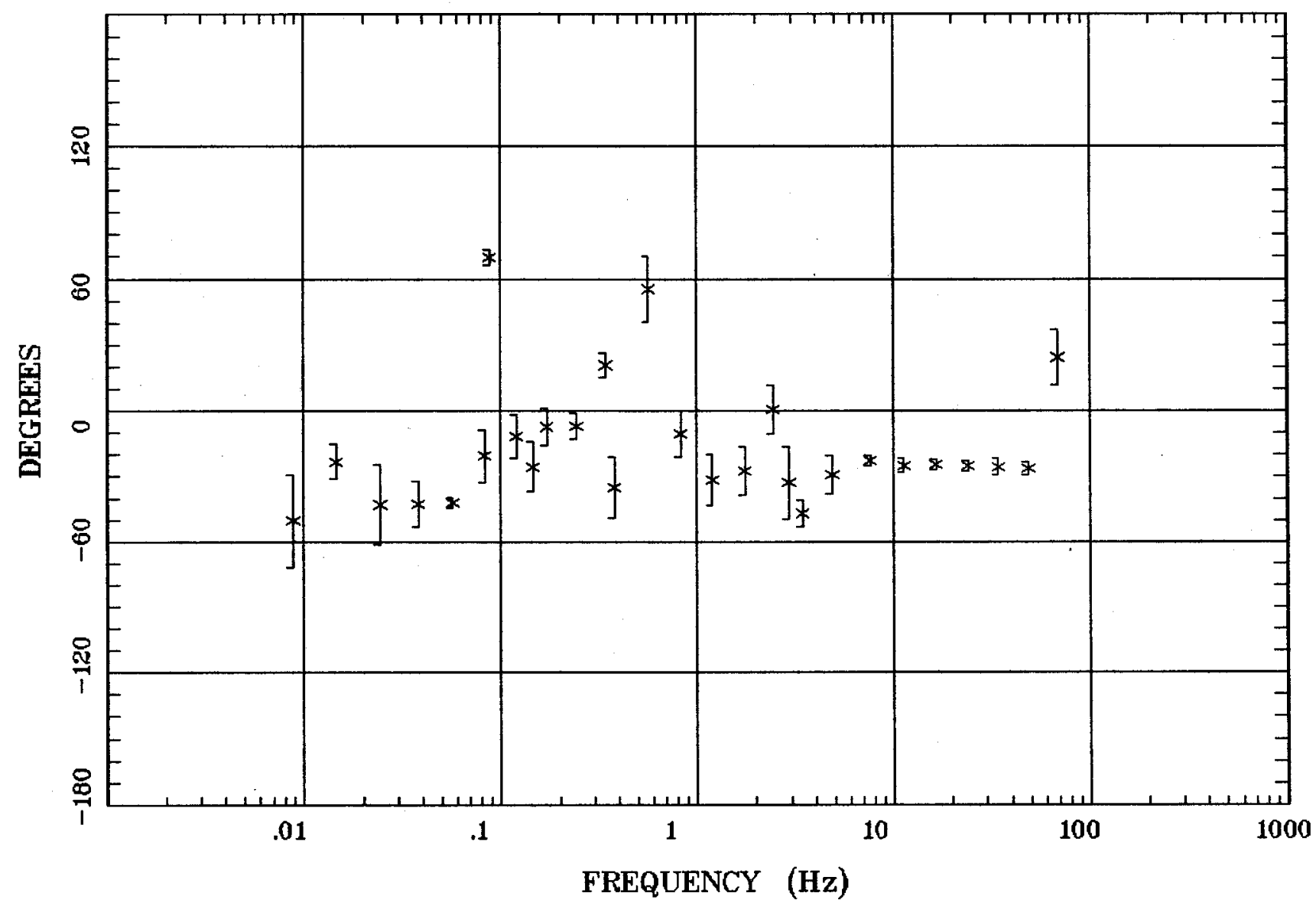

Client: Espanola Basin Remote: none Acquired: 10:1 Jun 07, 2004 Survey Co:USGS
Rotation:

Filename: cp36m5.avg

Channels: Ch1 Ch2 Ch3 Ch4 Ch5 Ch3 Ch4 Plotted: 15:11 Aug 04, 2004

< EMI - ElectroMagnetic Instruments > 
Station 36

\section{HzHx.x Coh HzHy.o Los Alamos, NM 100k}

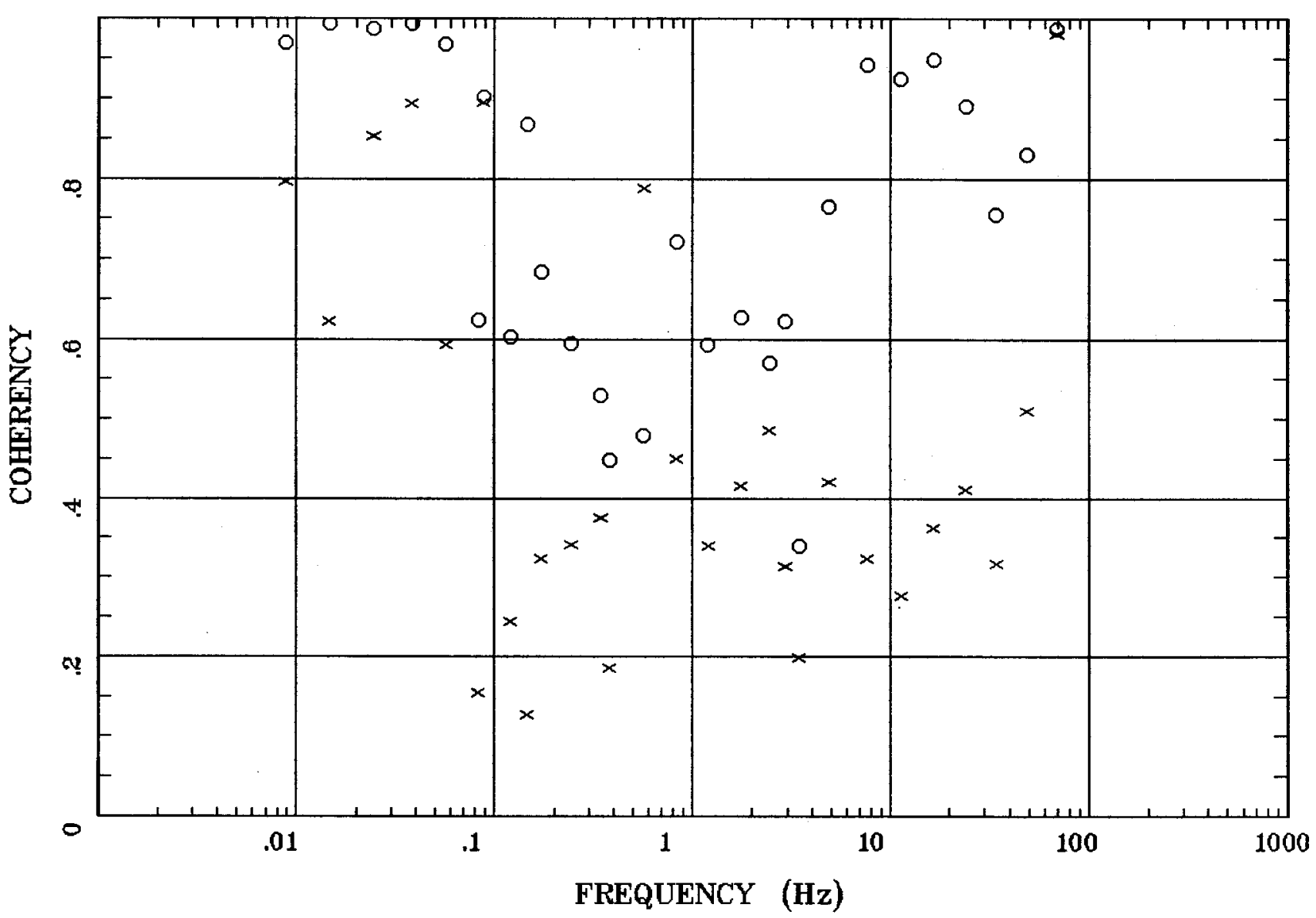

Client: Espanola Basin

Remote: none

Acquired: 10:1 Jun 07, 2004

Survey Co:USGS
Rotation:

Filename: cp36m5.avg

Channels: Ch1 Ch2 Ch3 Ch4 Ch5 Ch3 Ch4

Plotted: 15:11 Aug 04, 2004

$<$ EMI - ElectroMagnetic Instruments 


\section{APPARENT RESISTIVITY}

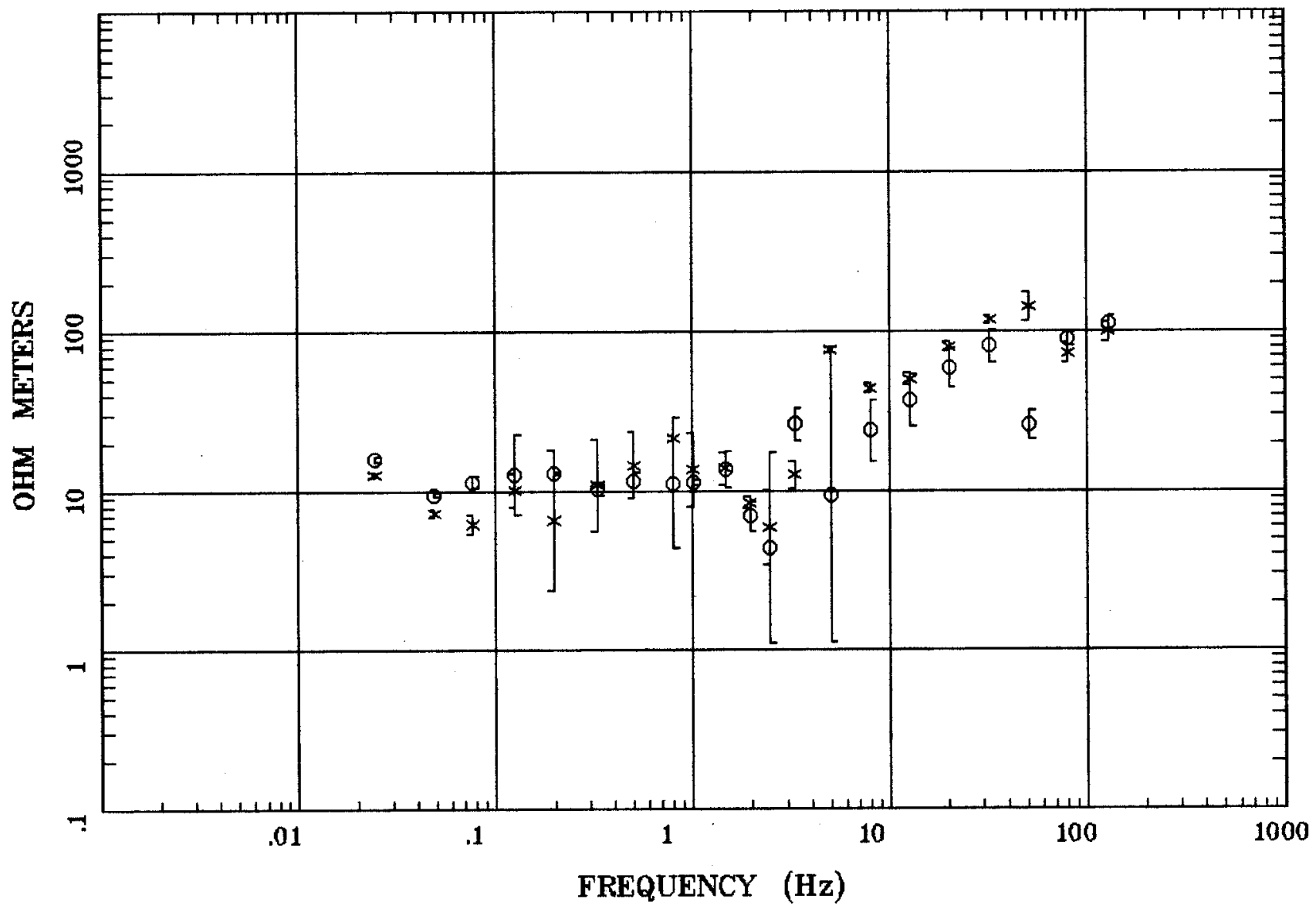

Client:

Remote:

Acquired: 19:5 Mar 08, 1998

Survey Co:
Rotation:

Filename: cp28.avg

Channels: Ch1 Ch2 Ch3 Ch4 Ch5 Ch3 Ch4 Plotted: 09:10 oct 22, 2004

< EMI - ElectroMagnetic Instruments 


\section{IMPEDANCE PHASE}

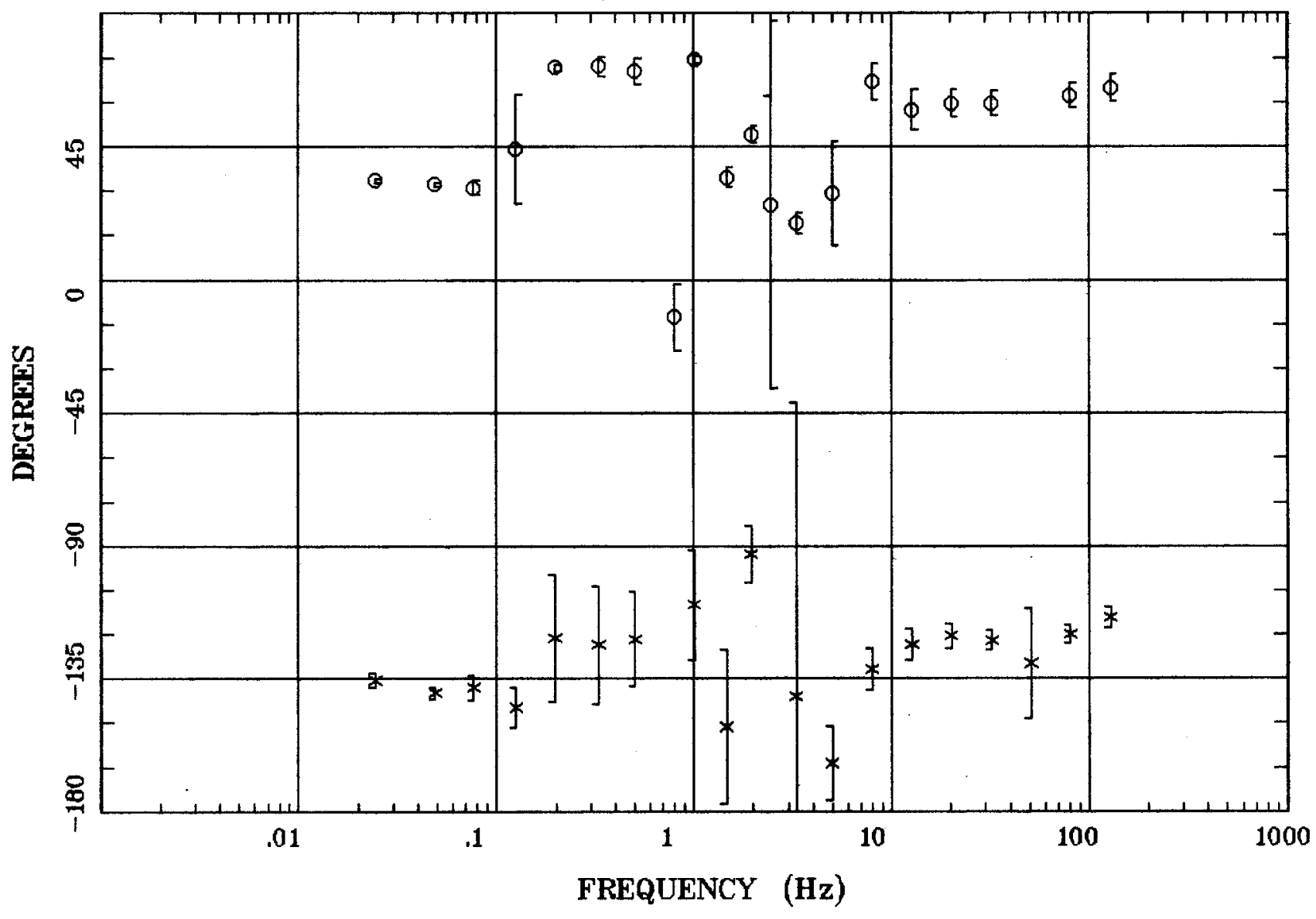

Client:

Remate:

Acquired: $19: 5$ Mar 08,1998 Survey Co:
Rotation:

Filename: cp28.avg

Channels: Ch1 Ch2 Ch3 Ch4 Ch5 Ch3 Ch4

Plotted: 09:10 oct 22, 2004

< EMI - ElectroMagnetic Instruments 
ROTATION ANGLE

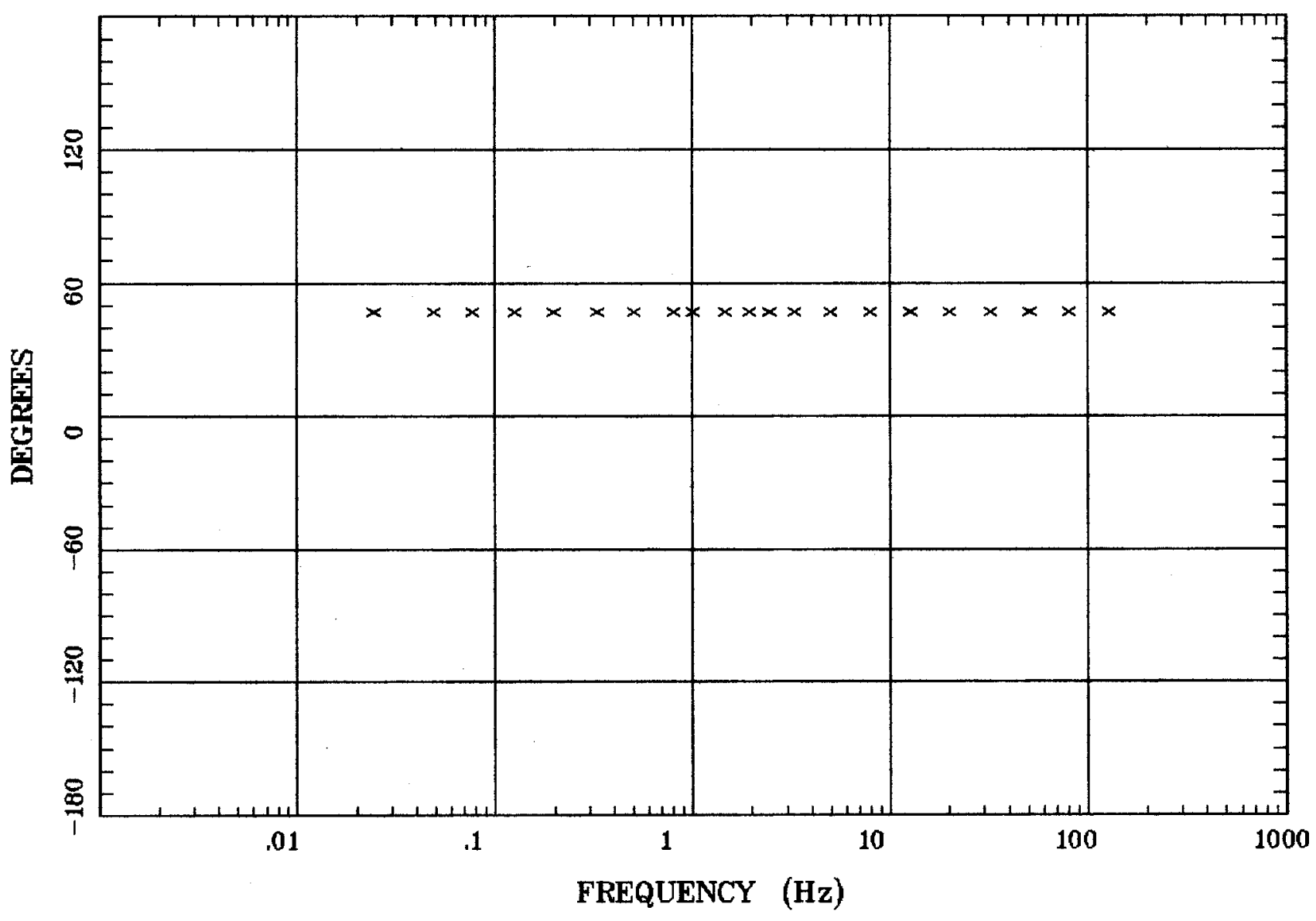

Client:

Remote:

Acquired: 19:5 Mar 08, 1998 Survey Co:
Rotation:

Filename: cp28.avg

Channels: Ch1 Ch2 Ch3 ch4 Ch5 Ch3 Ch4 Plotted: 09:10 oct 22, 2004

< EMI - ElectroMagnetic Instruments 


\section{IMPEDANCE SKEW}

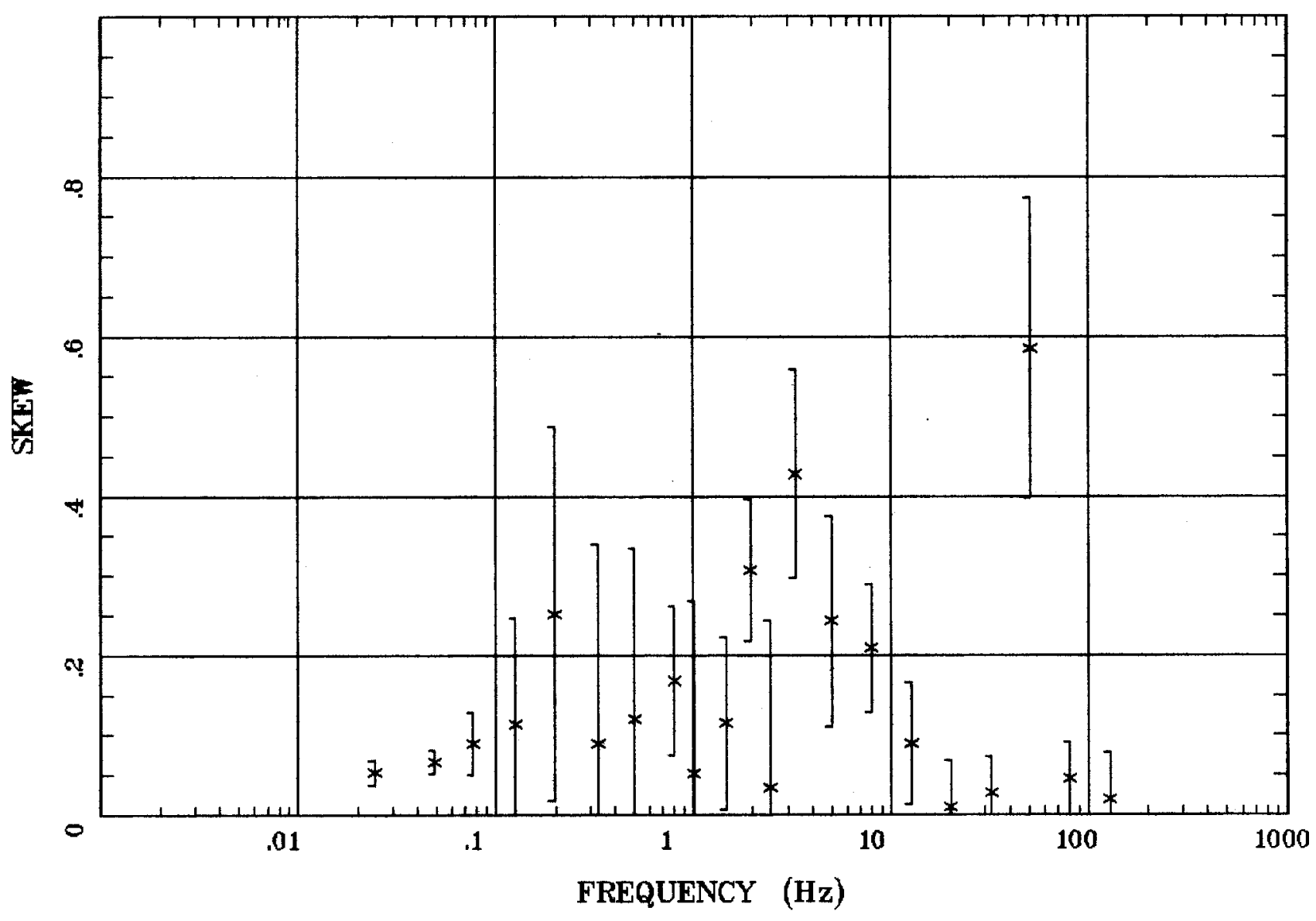

Client:

Remote:

Acquired: 19:5 Mar 08, 1998 Survey Co:
Rotation:

Filename: cpz8.avg

Channels: Ch1 Ch2 Ch3 Ch4 Ch5 Ch3 Ch4 Plotted: 09:10 oct 22, 2004

< EMI - ElectroMagnetic Instruments 
E MULT Coh.

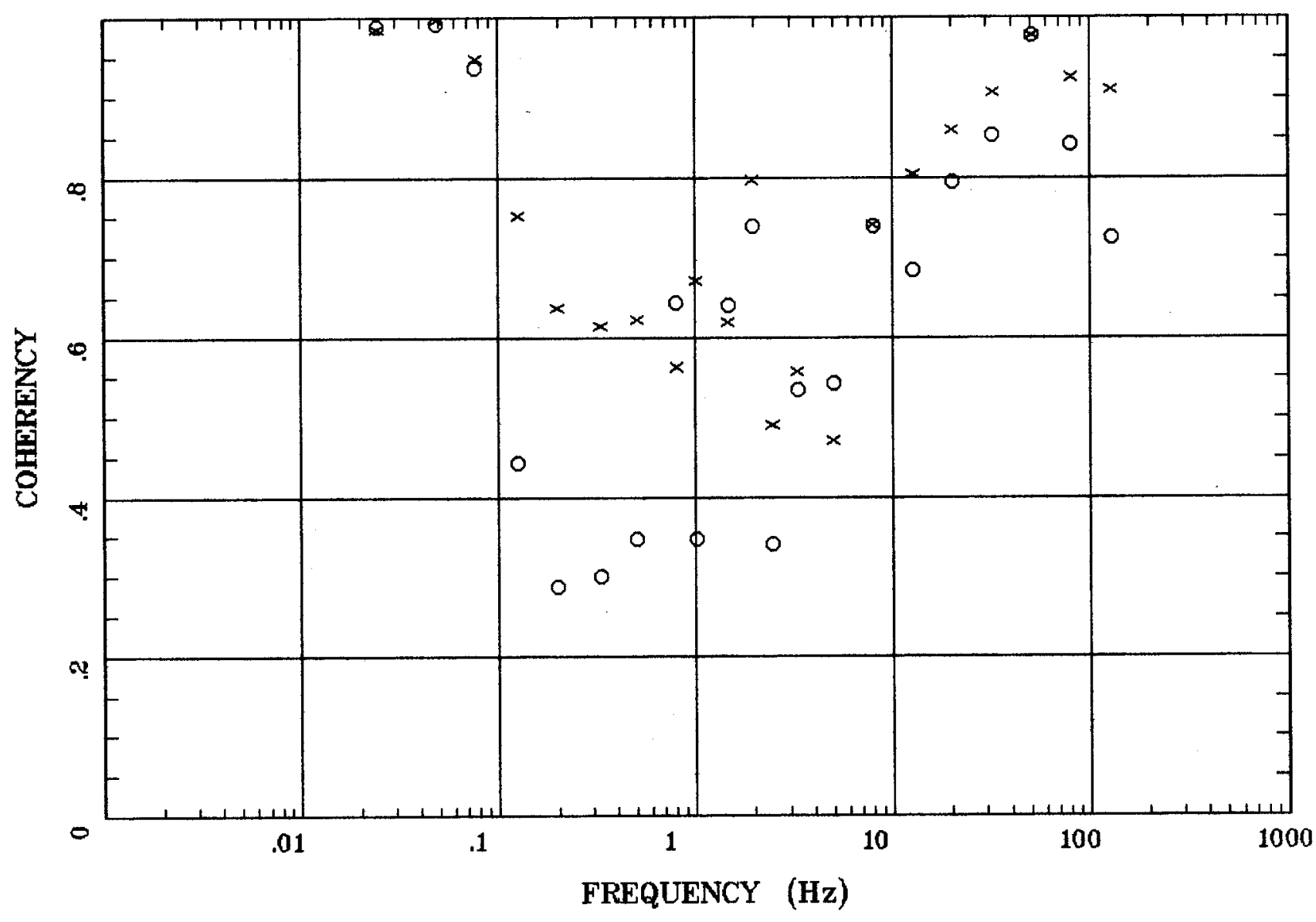

Client:

Remote:

Acquired: 19:5 Mar 08, 1998 Survey Ca:
Rotation:

Filename: cp28.avg

Channels: Ch1 Ch2 Ch3 Ch4 Ch5 Ch3 Ch4 Plotted: 09:10 Oct 22, 2004

$<$ EMI - ElectroMagnetic Instruments 


\section{POLAR PLOTS}

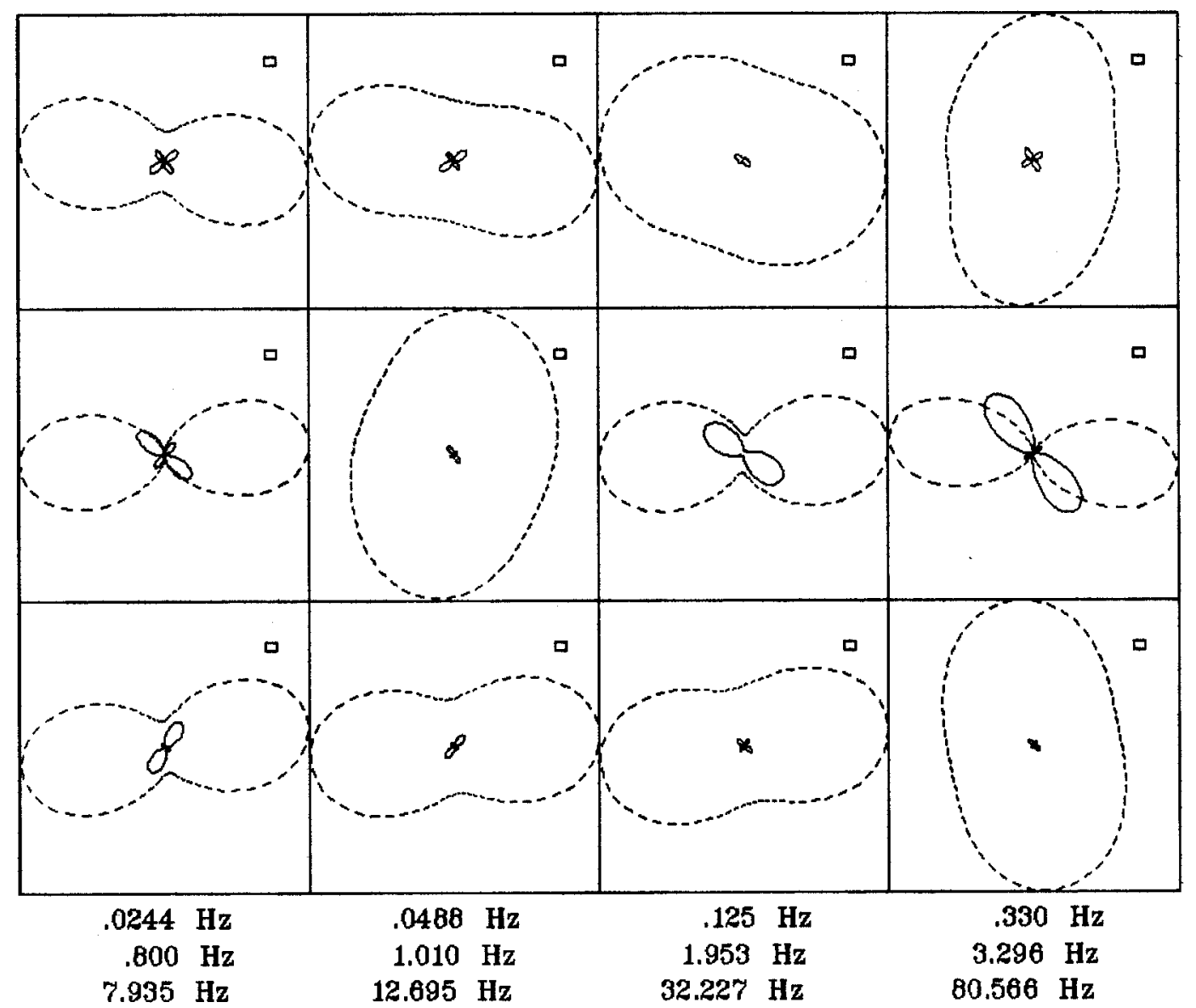

Client:

Remote:

Acquired: 19:5 Mar 08, 1998

Survey Ca:
Rotation:

Filename: cp28.evg

Channels: Ch1 Ch2 Ch3 Ch4 Ch5 Ch3 Ch4 Plotted: 09:10 oct 22, 2004

< EMI - ElectroMagnetic Instruments > 
TIPPER MAGNITUDE

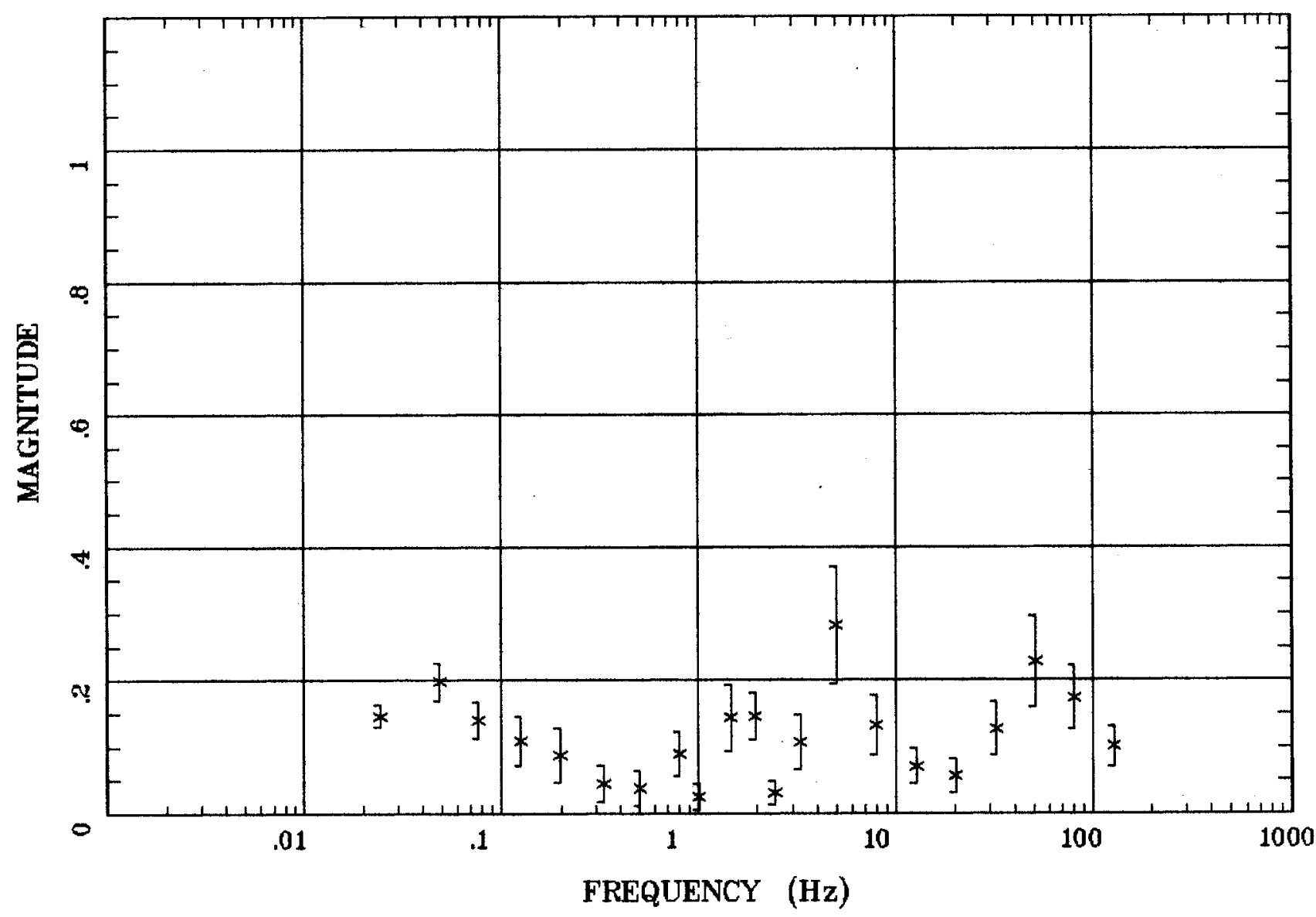

Client:

Remote:

Acquired: 19:5 Mar 08, 1998

Survey Co:
Rotation:

Filename: cp28.avg

Channels: Ch1 Ch2 Ch3 Ch4 Ch5 Ch3 Ch4

Plotted: 09:10 oct 22, 2004

< EMI - ElectroMagnetic Instruments 


\section{Station 28}

\section{TIPPER STRIKE}

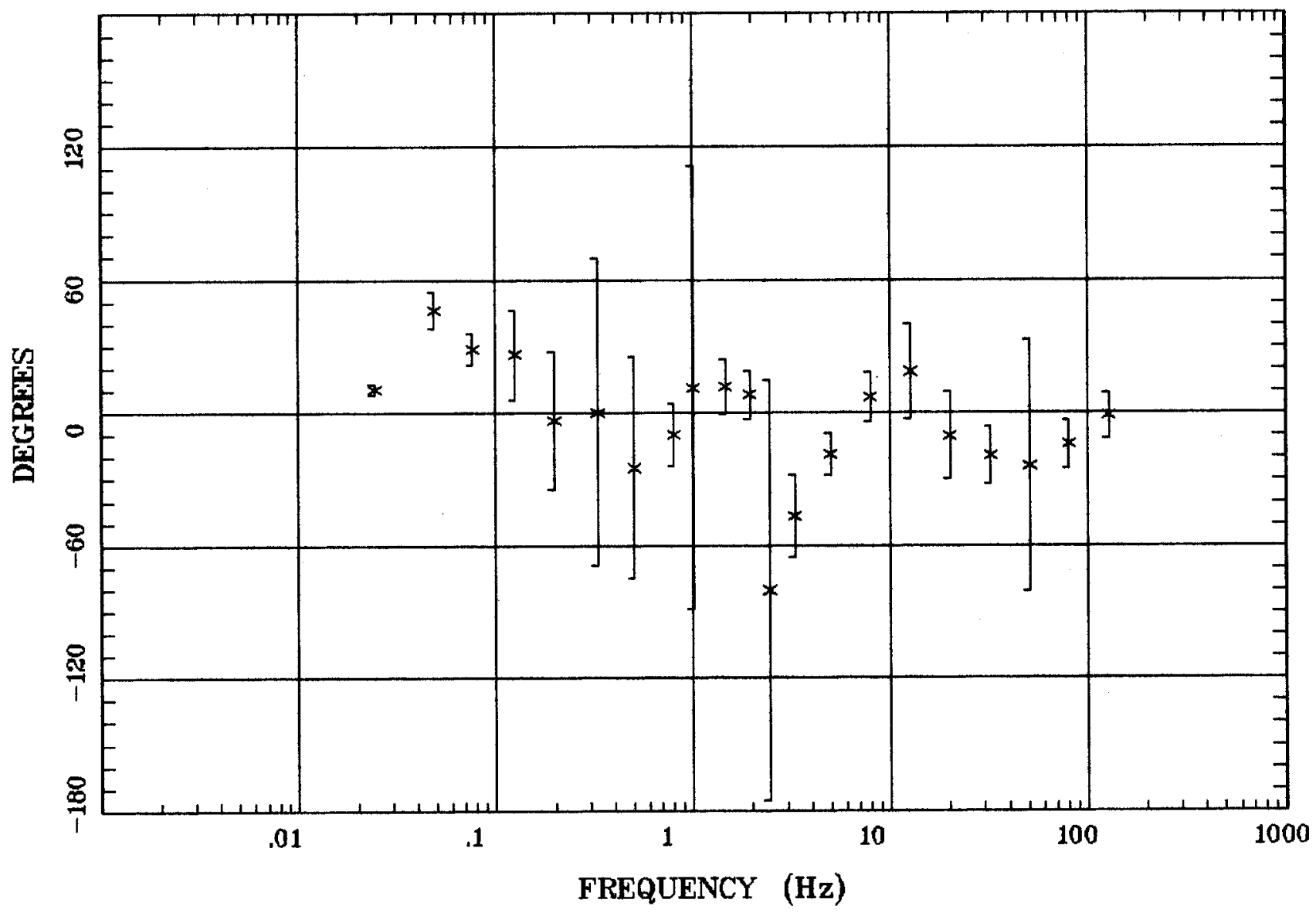

Client:

Remate:

Acquired: 19:5 Mar 08, 1998 Survey Co:
Rotation:

Filename: cp28.avg

Channels: Ch1 ch2 Ch3 Ch4 Ch5 Ch3 Ch4 Plotted: 09:10 oct 22, 2004

< EMI - ElectroMagnetic Instruments > 
Station 28

HzHx.x Coh HzHy.o

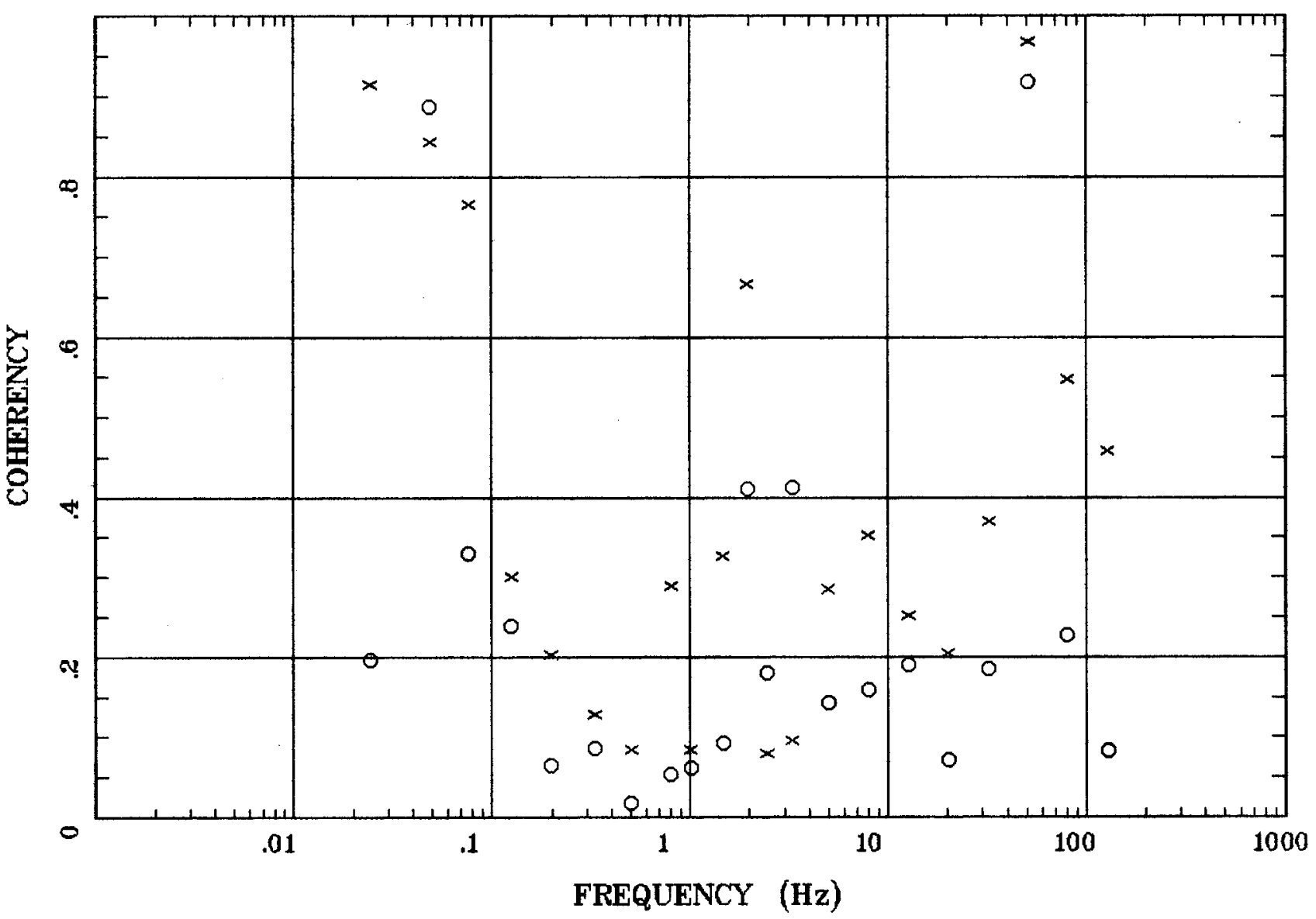

Client:

Remote:

Acquired: 19:5 Mar 08, 1998

Survey Co:
Rotation:

Filename: cp28.avg

Channels: Ch1 Ch2 Ch3 Ch4 Ch5 Ch3 Ch4 Plotted: 09:10 oct 22, 2004

< EMI - ElectroMagnetic Instruments > 


\section{Station 29}

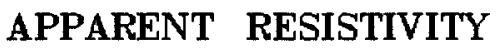

Los Alamos, NM 100k

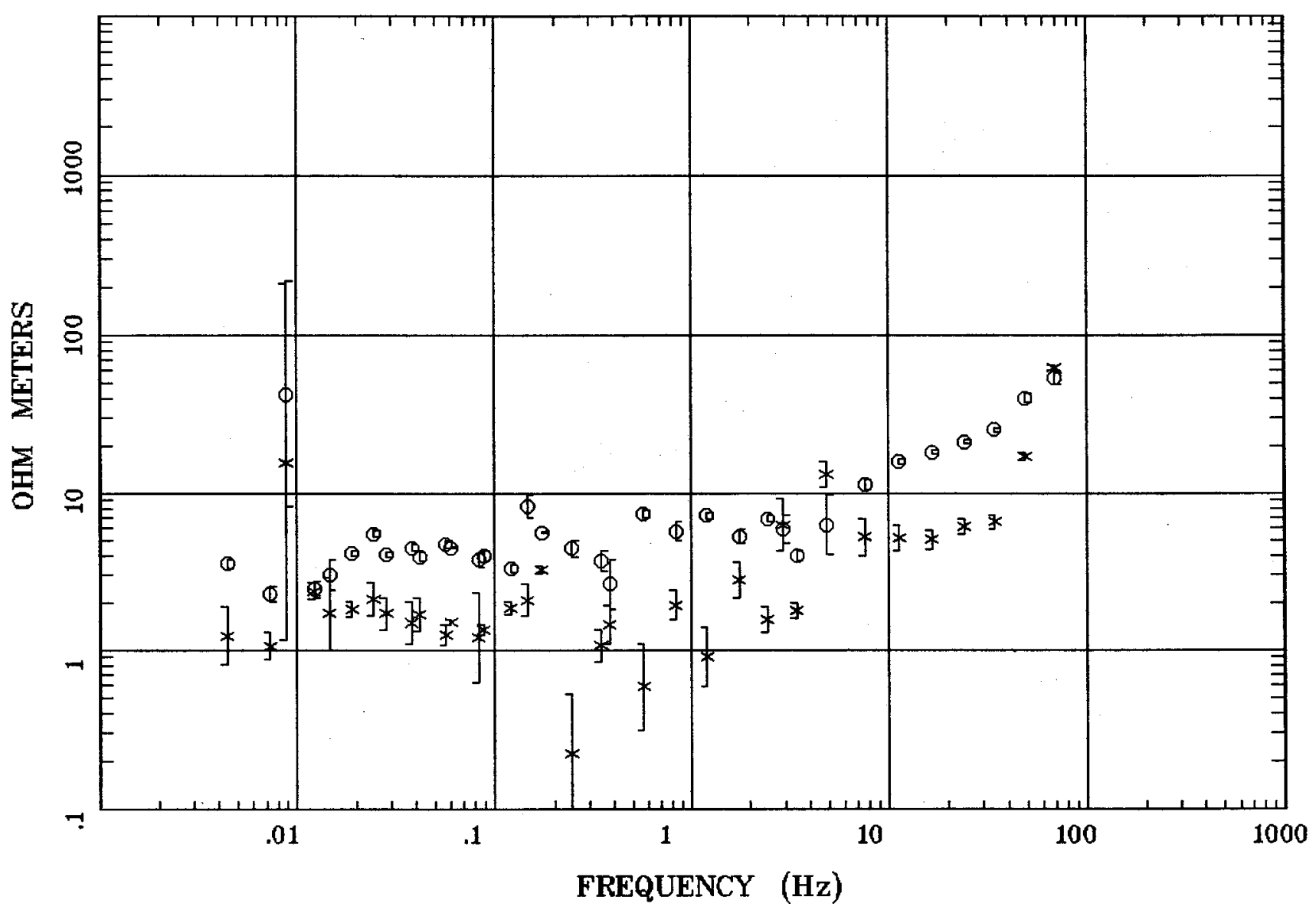

Client: Espanola Basin Remote: none

Acquired: 11:1 Jun 06, 2004 Survey Co:USGS
Rotation:

Filename: cp29mb.avg

Channels: Ch1 Ch2 Ch3 Ch4 Ch5 Ch3 Ch4 Plotted: 14:08 Aug 18, 2004

< EMI - ElectroMagnetic Instruments > 


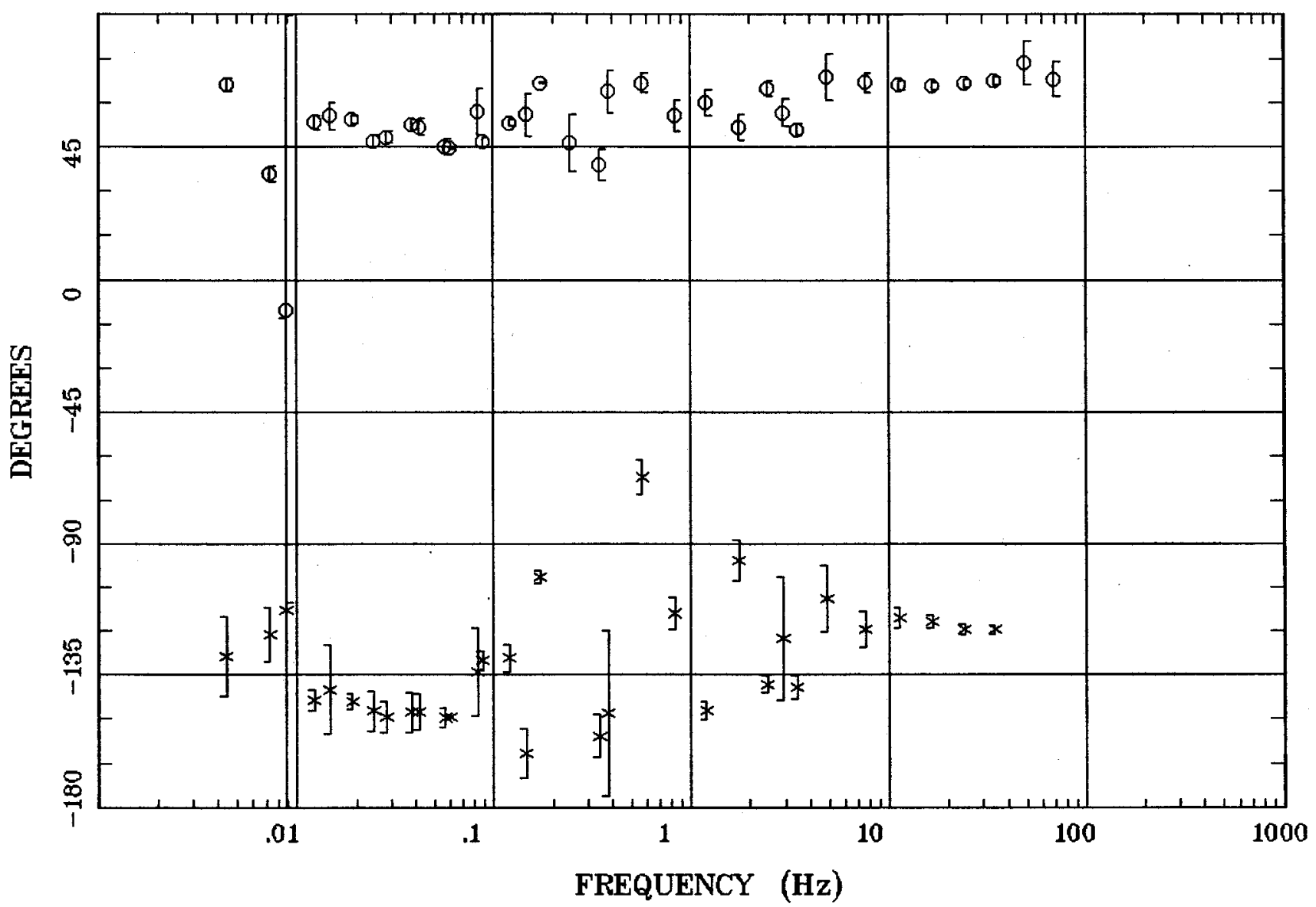

Client: Espanola Basin Remote: none Acquired: 11:1 Jun 06, 2004 Survey Co:USGS
Rotation:

Filename: $c p 29 \mathrm{mb}$.avg

Channels: Ch1 Ch2 Ch3 Ch4 Ch5 Ch3 Ch4 Plotted: 14:08 Aug 18, 2004

< EMI - ElectroMagnetic Instruments > 


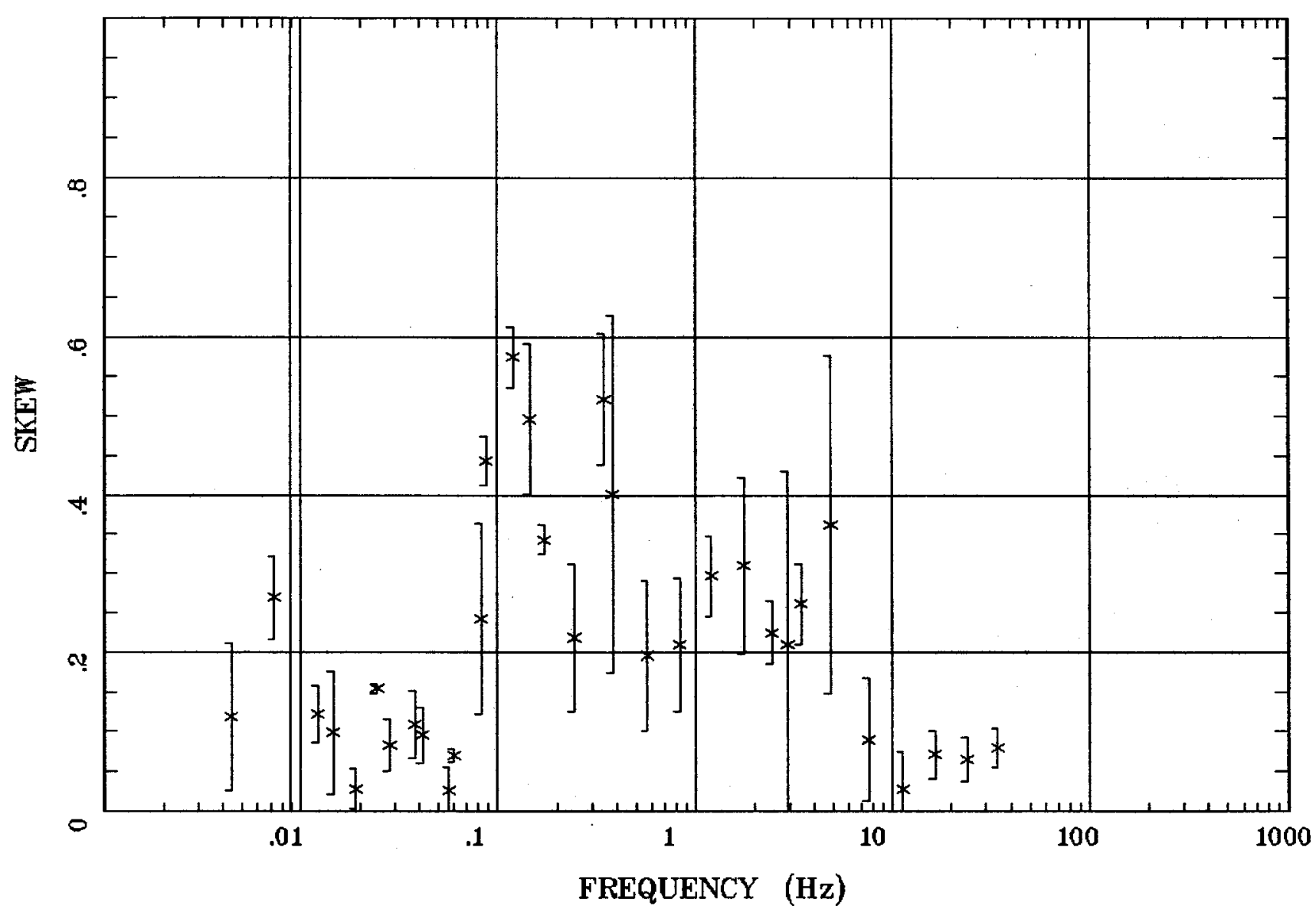

Client: Espanola Basin Remote: none Acquired: 11:1 Jun 06, 2004 Survey Co:USGS
Rotation:

Filename: cp29mb.avg

Channels: Ch1 Ch2 Ch3 Ch4 Ch5 Ch3 Ch4 Plotted: 14:08 Aug 18, 2004

$<$ EMI - ElectroMagnetic Instruments 
E MULT Coh.

Los Alamos, NM 100k

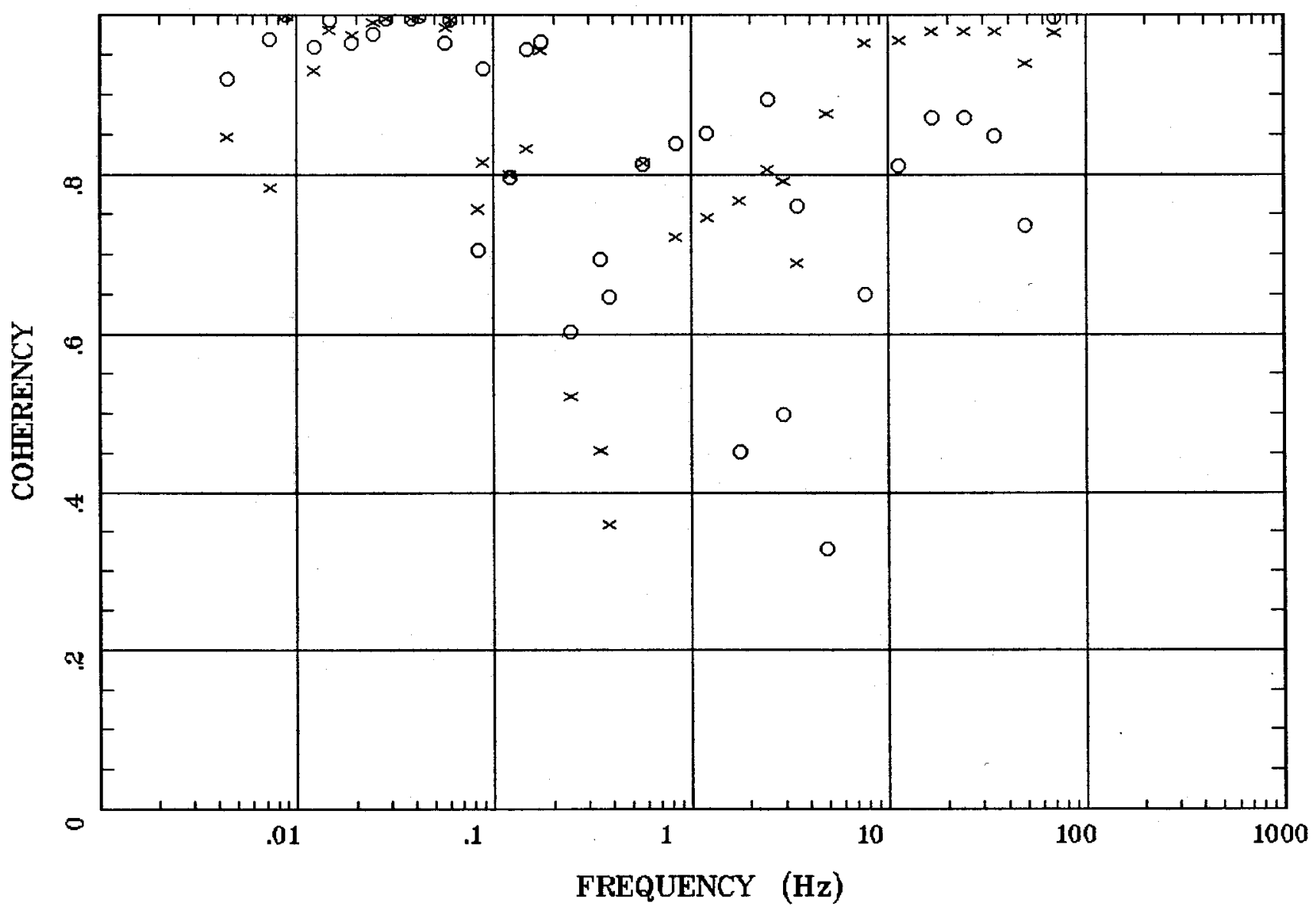

Client: Espanola Basin

Remote: none

Acquired: 11:1 Jun 06, 2004

Survey Co:USGS
Rotation:

Filename: cp29mb.avg

Channels: Ch1 Ch2 Ch3 Ch4 Ch5 Ch3 Ch4

Plotted: 14:08 Aug 18, 2004

$<$ EMI - ElectroMagnetic Instruments 
POLAR PLOTS

Los Alamos, NM 100k

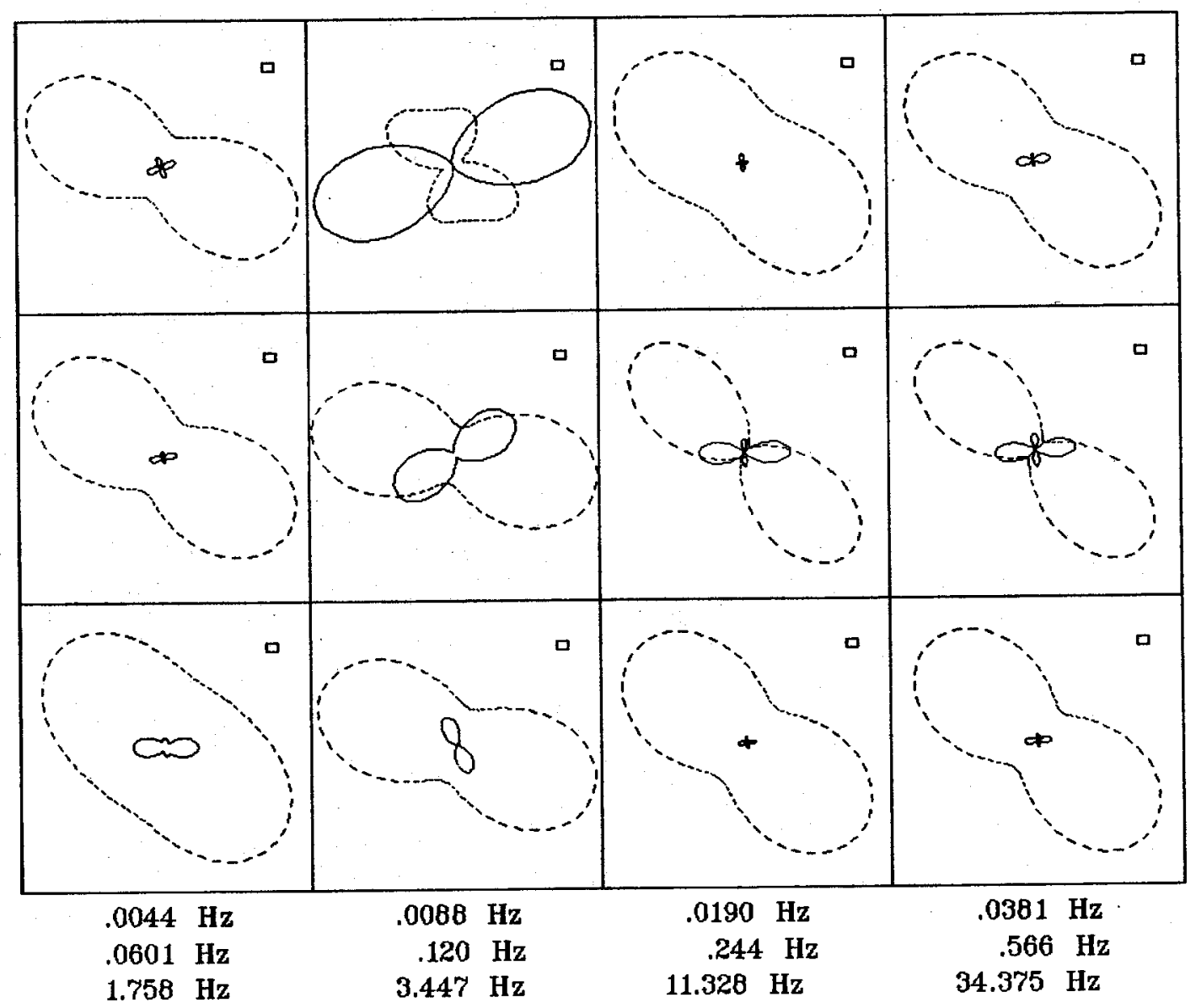

Rotation:

Client: Espanola Basin Remote: none Acquired: 11:1 Jun 06, 2004 Survey Co:USGS
Filename: cp29mb.avg

Channels: Ch1 Ch2 Ch3 Ch4 Ch5 Ch3 Ch4 Plotted: 13:25 Aug 12, 2004

< EMI - ElectroMagnetic Instruments > 


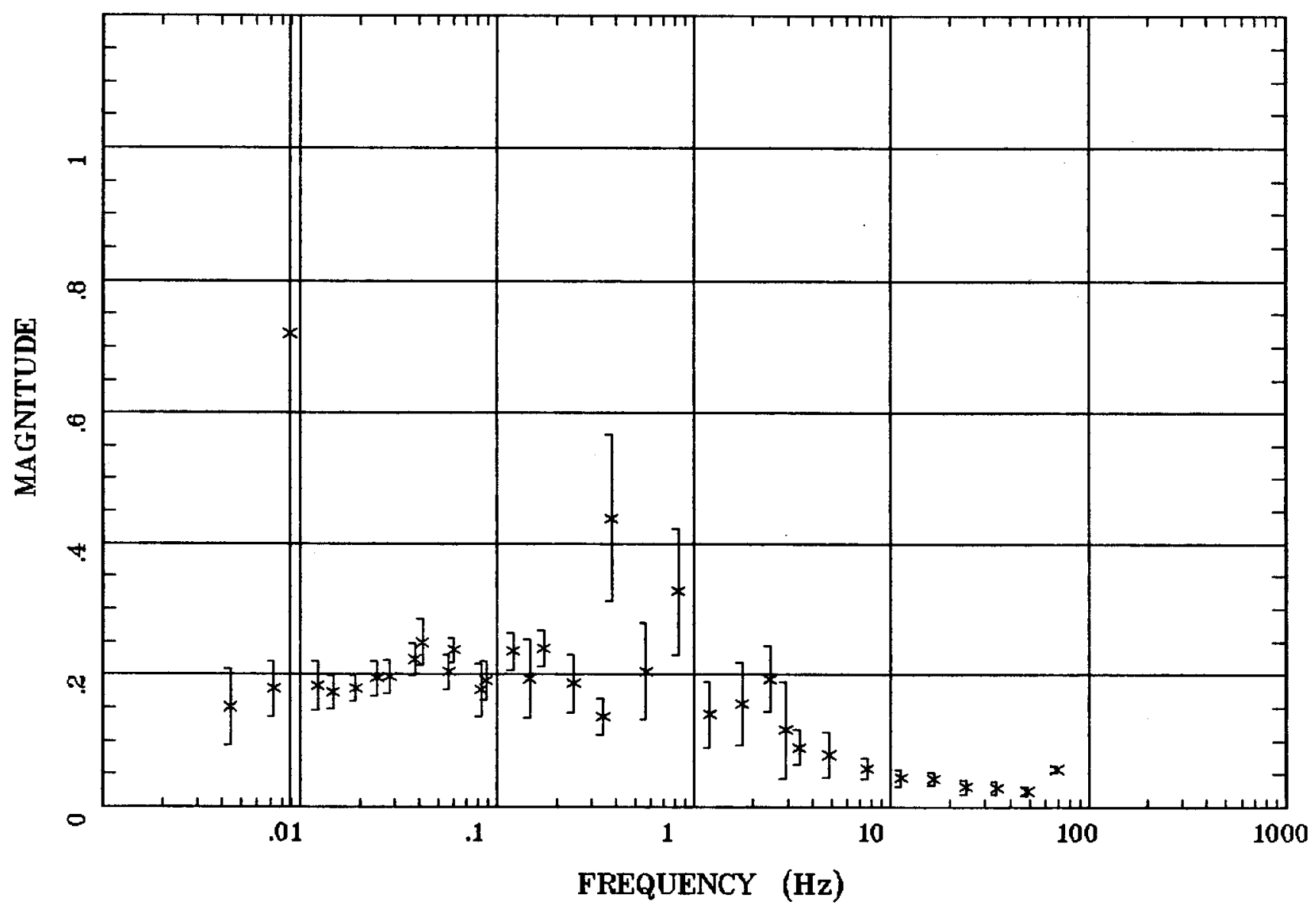

Client: Espanola Basin Remote: none Acquired: 11:1 Jun 06, 2004 Survey Co:USGS
Rotation:

Filename: cp29mb.avg

Channels: Ch1 Ch2 Ch3 Ch4 Ch5 Ch3 Ch4 Plotted: 14:08 Aug 18, 2004

< EMI - ElectroMagnetic Instruments 


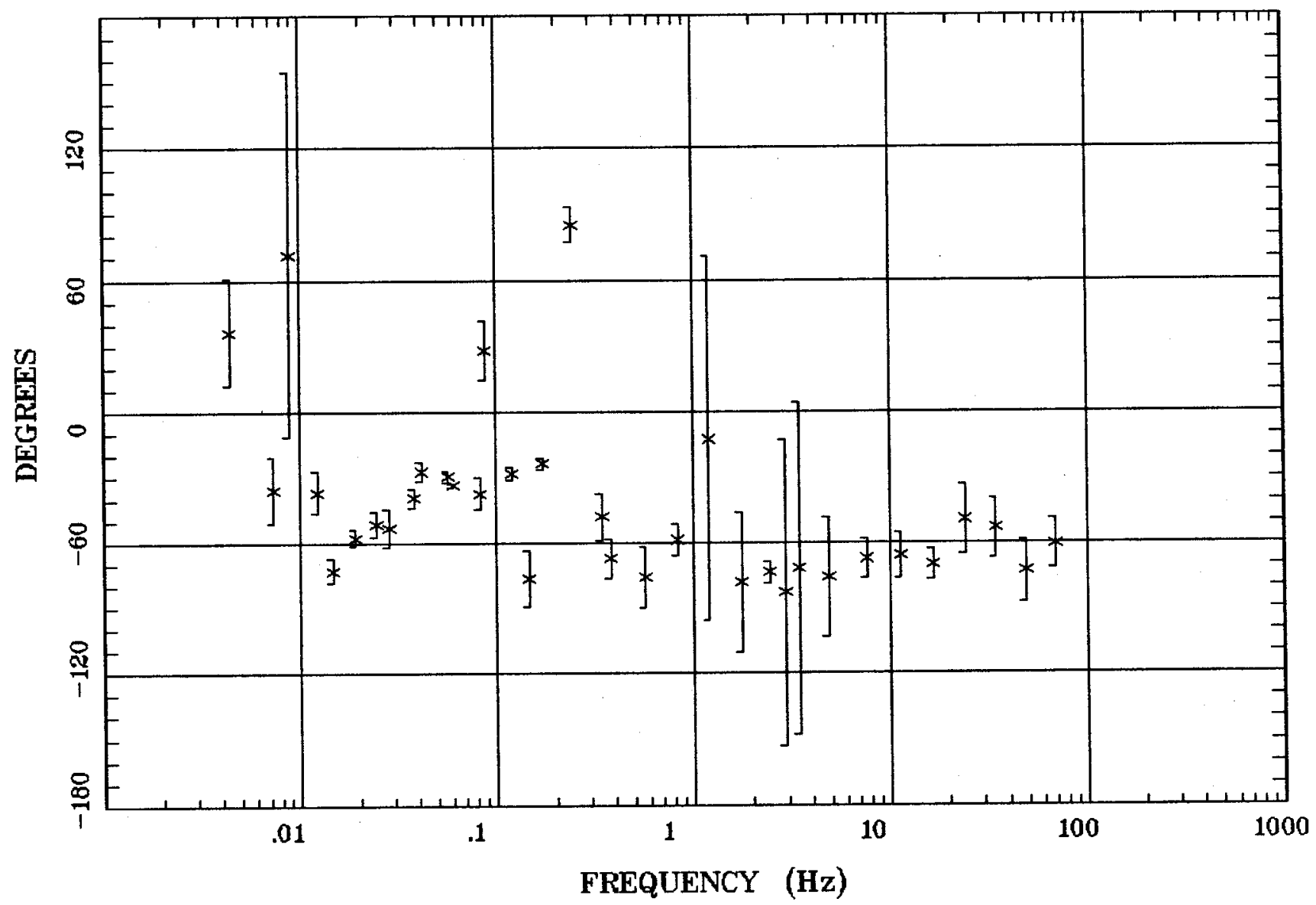

Client: Espanola Basin Remote: none Acquired: 11:1 Jun 06, 2004 Survey Co:USGS

\section{Rotation:}

Filename: cp29mb.avg

Channels: Ch1 Ch2 Ch3 Ch4 Ch5 Ch3 Ch4 Plotted: 14:08 Aug 18, 2004

< EMI - ElectroMagnetic Instruments 
HzHx.x Coh HzHy.o

Los Alamos, NM 100k

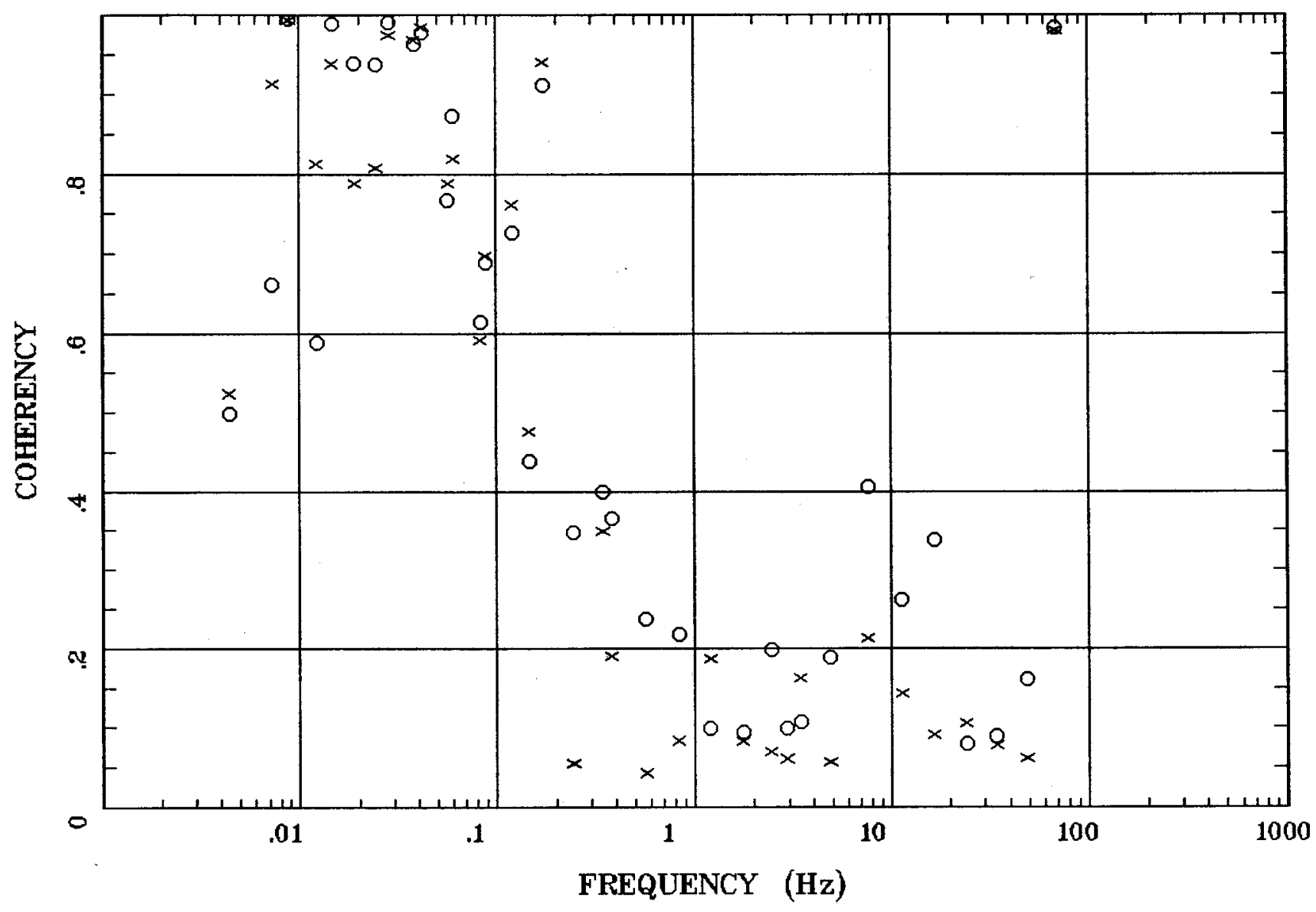

Client: Espanola Basin

Remote: none

Acquired: 11:1 Jun 06, 2004

Survey Co:USGS
Rotation:

Filename: cp29mb.avg

Channels: Ch1 Ch2 Ch3 Ch4 Ch5 Ch3 Ch4

Plotted: 14:08 Aug 18, 2004

< EMI - ElectroMagnetic Instruments > 